\title{
Deep Coverage Proteomics Identifies More Low-Abundance Missing Proteins in Human Testis Tissue with Q-Exactive HF Mass Spectrometer
}

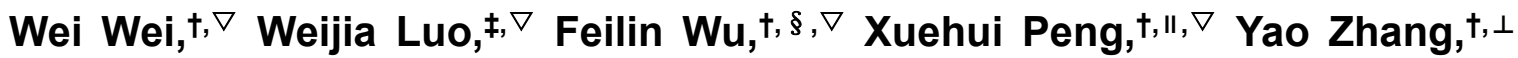
Manli Zhang, ${ }^{\dagger}$ Yan Zhao, ${ }^{\dagger} \mathrm{Na}$ Su, ${ }^{\dagger}$ YinZi Qi, ${ }^{\dagger}$ Lingsheng Chen, ${ }^{\dagger}$ Yangjun Zhang, ${ }^{\dagger}$ Bo Wen, ${ }^{\#}$ Fuchu He, ${ }^{*, \dagger}$ Ping $X u^{*,+, \neq, \|}$

tState Key Laboratory of Proteomics, National Center for Protein Sciences Beijing, Beijing Proteome Research Center, National Engineering Research Center for Protein Drugs, Beijing Institute of Radiation Medicine, Beijing 102206, China

¥Graduate School, Anhui Medical University, Hefei 230032, China

sLife Science College, Southwest Forestry University, Kunming, 650224, China

"Key Laboratory of Combinatorial Biosynthesis and Drug Discovery of the Ministry of Education, School of Pharmaceutical Sciences, Wuhan University, Wuhan 430072, China

$\perp$ Institute of Microbiology, Chinese Academy of Science, Beijing 100101, China

\#BGI-Shenzhen, Shenzhen 518083, China

$\nabla$ These authors contributed equally to this work.

* To whom correspondence should be addressed:

Ping Xu, Tel/Fax: 86-10-80705066; E-mail: xuping_bprc@126.com

Fuchu He, Tel/Fax: 86-10-68171208; E-mail: hefc@nic.bmi.ac.cn

Supporting information file:

Figure S1-6, and Table S1-3. 


\section{Supplementary Figure Legends}

Figure S-1. Molecular weight distribution of proteins identified by Glycineand Tricine-SDS-PAGE.

Figure S-2. Comparison of identified proteins from three individual testis tissues based on two gel-separation methods.

(A) Venn diagram of proteins of three individual testis tissues based on Glycine SDS-PAGE. (B) Venn diagram of proteins of three individual testis tissues based on Tricine SDS-PAGE.

Figure S-3. Comparison of identified proteins from three individual testis tissues based on two separation ways by HF and Velos.

(A) Venn diagram of proteins of three individual testis tissues based on Glycine SDS-PAGE by HF and Velos. (B) Venn diagram of three individual testis tissues based on Tricine SDS-PAGE in HF and Velos.

Figure S-4. The number and sequence coverage of testis MPs.

(A) Venn diagram of MPs in three datasets. (B) The sequence coverage of MPs in HF and Velos datasets, and the percent presents that the number of identified unique peptide of MPs accounting for total identified MPs.

Figure S-5. The MW and pl of identified proteins by HF and Velos.

(A) The distribution of MW of uniquely identified proteins by HF and Velos.

(B) The distribution of $\mathrm{pl}$ of uniquely identified proteins by HF and Velos.

Figure S-6. The verification of MPs by its synthesized peptide and previous MS spectra matching. 
Figure S-1

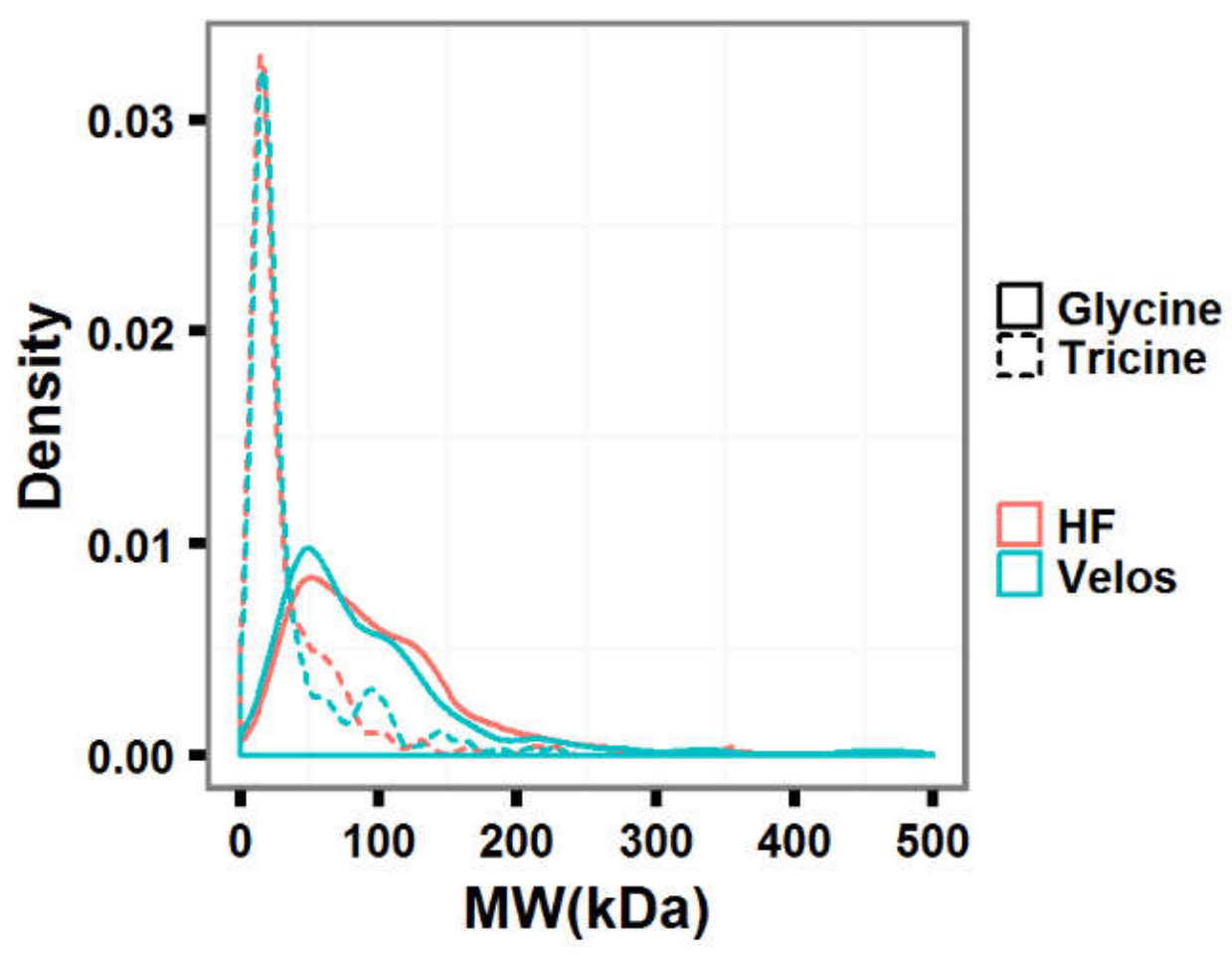




\section{Figure S-2}

A

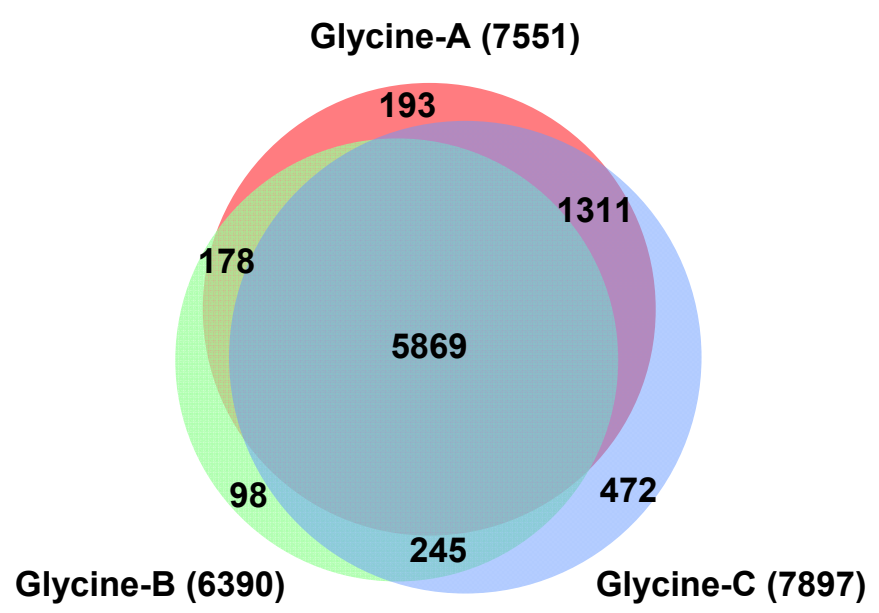

B

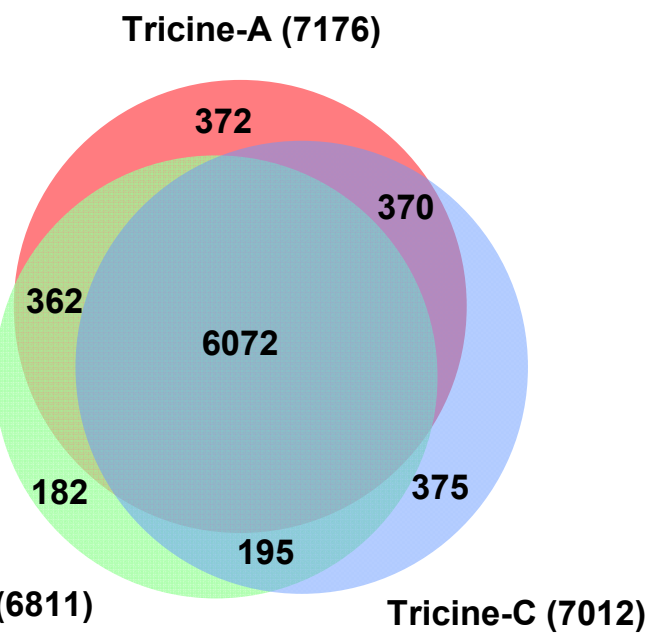




\section{Figure S-3}

A

Velos-SDS-A

(6644)

HF-SDS-A

(7551)

260

6384

1167

HF-SDS-B

(6390)

(6351)

916

5435

955

HF-SDS-C

(7897)

(6660)

Velos-SDS-C

235

6425
B

Velos-Tricine-A

(6173)

HF-Tricine-A

(7176)

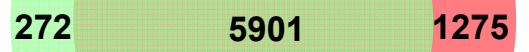

Velos-Tricine-B

(6093)

HF-Tricine-B

(6811)

353

5740

1071
Velos-Tricine-C

(6182)

HF-Tricine-C (7012)

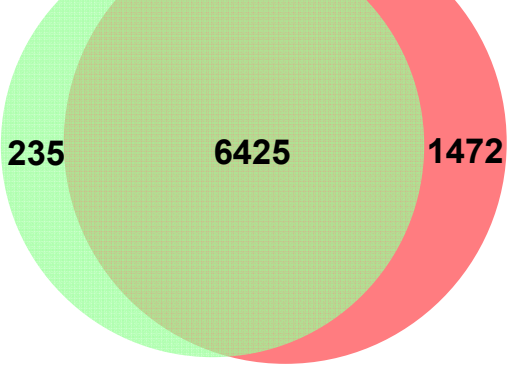

372

5810 
Figure S-4

A

HF_MPs

(74)

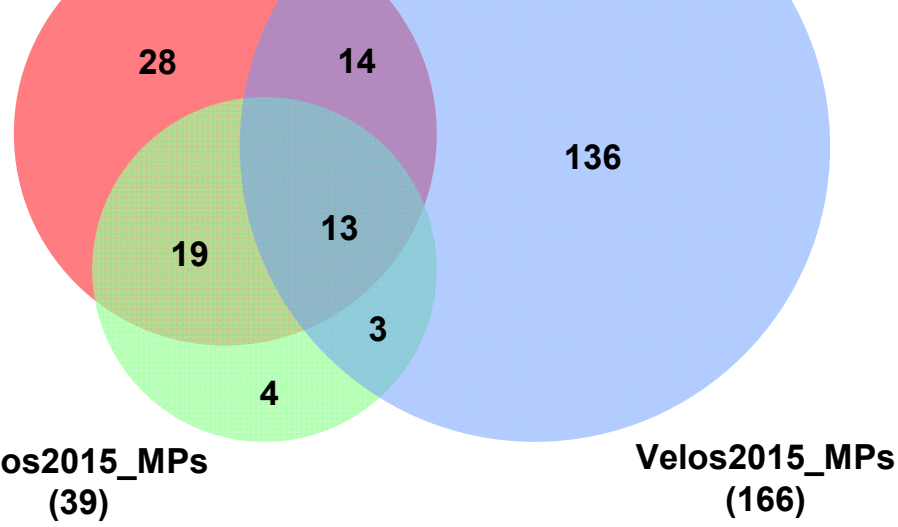

B

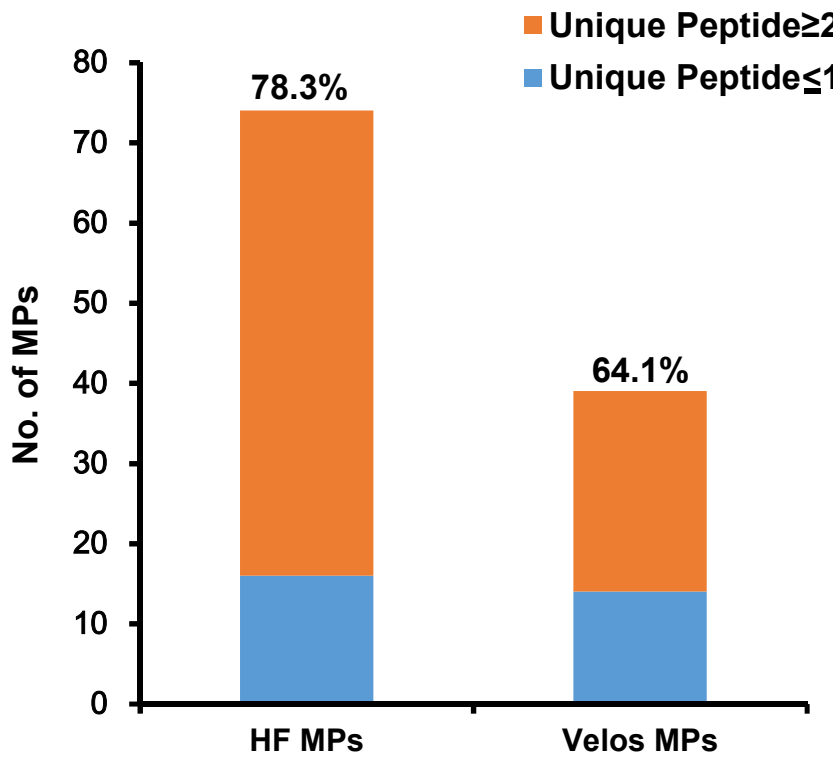


Figure S-5

A

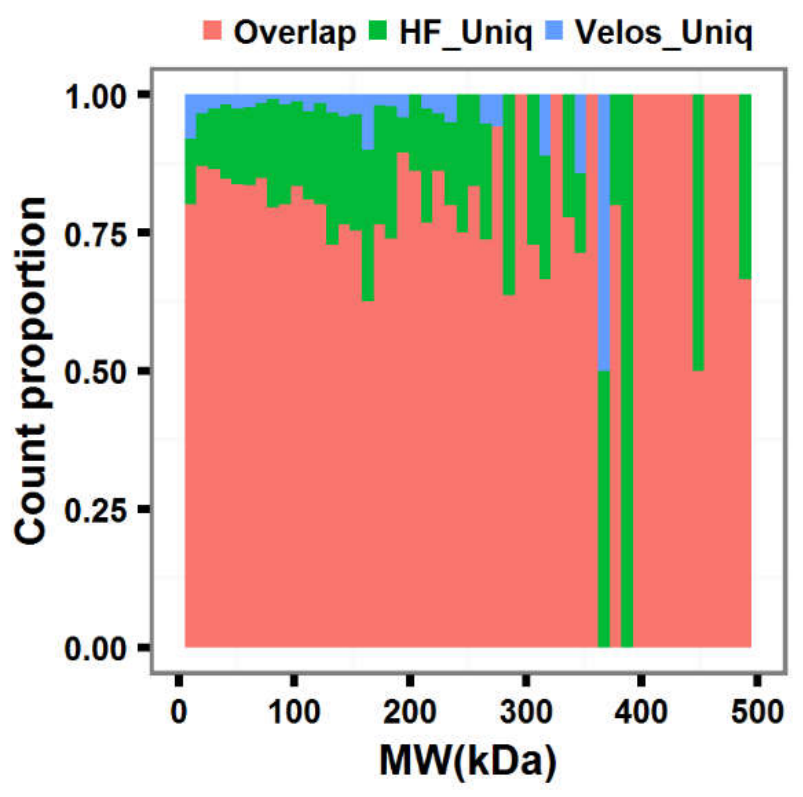

B

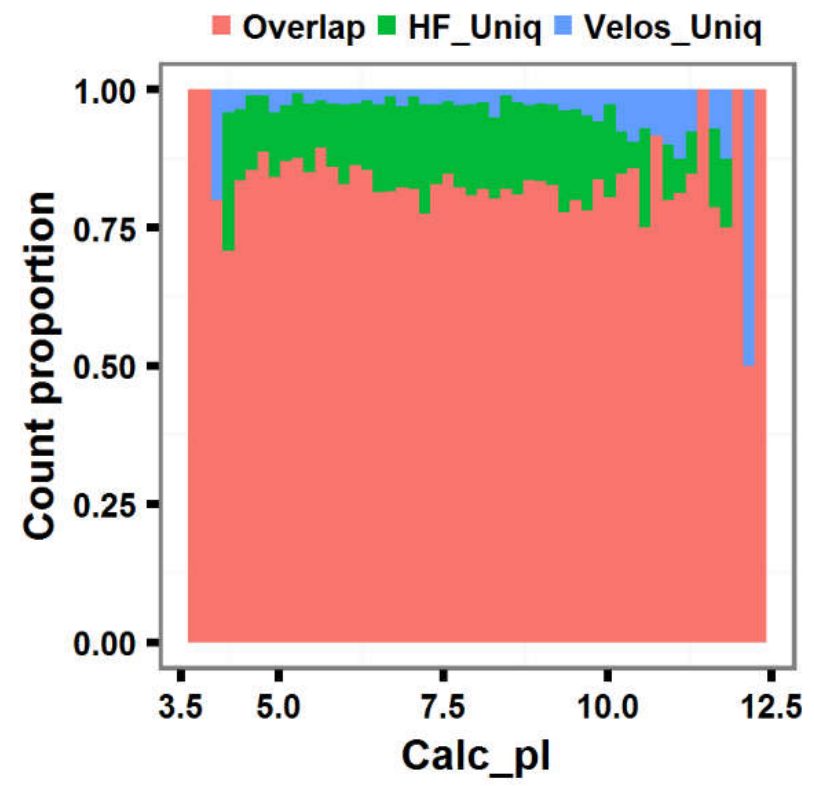




\section{Figure S-6}

\section{FIAFCGPHSR}

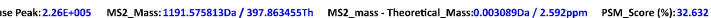

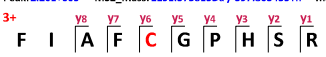

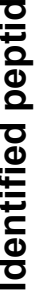

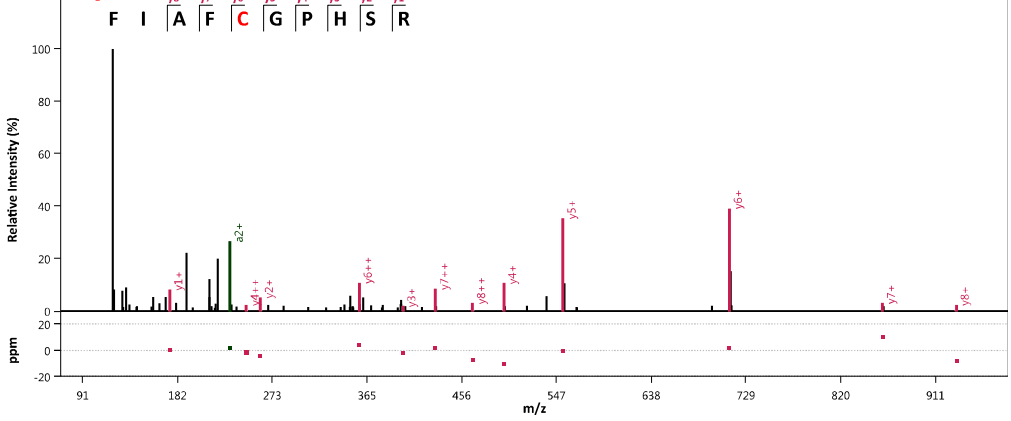

Title: 4817_HF01_XP_CHPP_peptide_Test_5ul_60ms_AGC2E4.16744.16744.3.0.dta Mods: 5,Carbamidomethyl[C](None); Label: None Info: 4817_HF01_XP_cHPP_peptide_Test_5ul_60ms_AGC2 Base Peak:2.51E+006 MS2_Mass: 1191.5740360a / 397.862863Th MS2_mass - Theoretical_Mass:0.001312Da/ 1.101ppm PSM_Score (\%):43.511

के

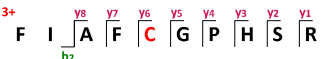
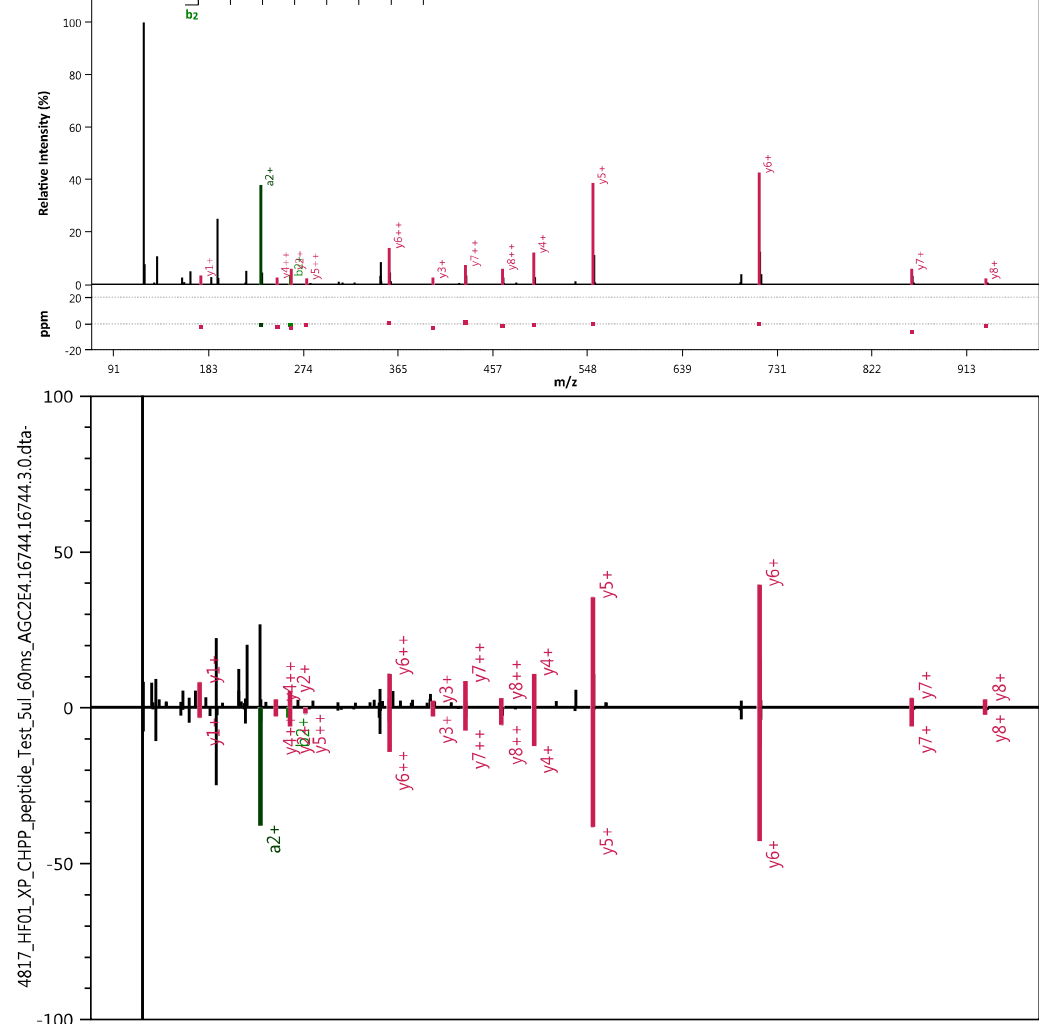
Titele:4817_HF01_XP_CHPP_Has_SDS10_15_5ul_60ms_AGC2E4.36680.36680.3.0.dta Mods:2,Carbamidomethyl[CIINone); Label: None Info: 4817_HF01_XP_CHPP_Has_SDS10_15_SUl_60ms_AC Base Peak:5.34F+004 MS2_Mass: 2084.1554470a / 695.39Th MS2_mass - Theoretical_Mass:0.0048120a / 2.309ppm PSM_Score (\%): 33.595

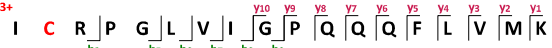

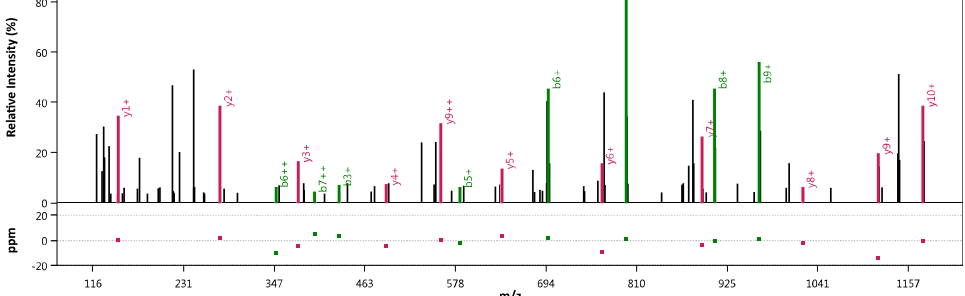

के

Titele:4817_HF01_XP_CHPP_peptide_Test_SUl_60ms_AGC2E4.30756.30756.3.0.dta Mods: 2,Carbamidomethyl[C]INone); Label: None Info: 4817_H

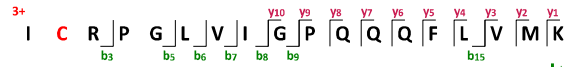
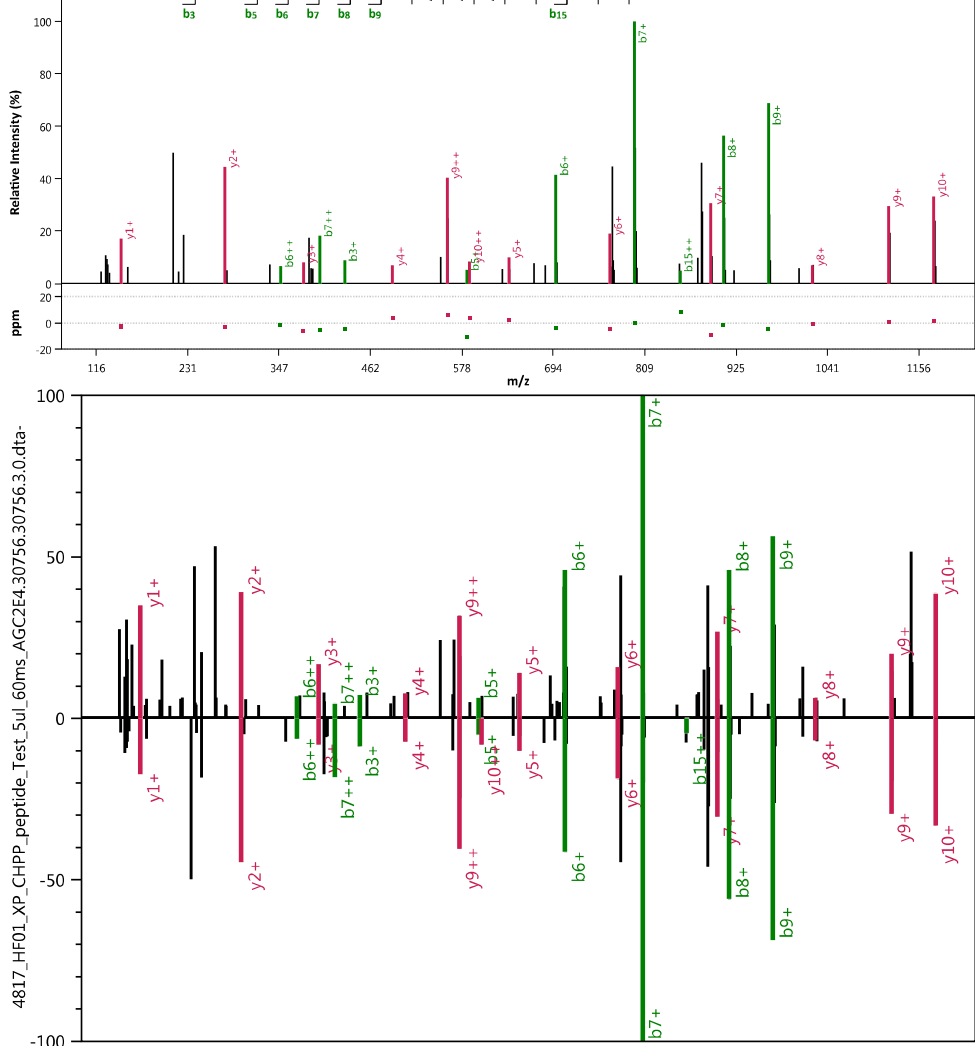


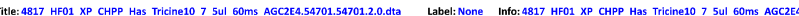
Base Peak: 1.42E+005 MS2_Mass: 1551.861274Da/ 776.434275Th MS2_mass - Theoretical_Mass:0.004366Da/2.814ppm PSM_Score (\%):37.078

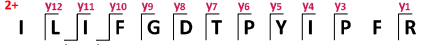

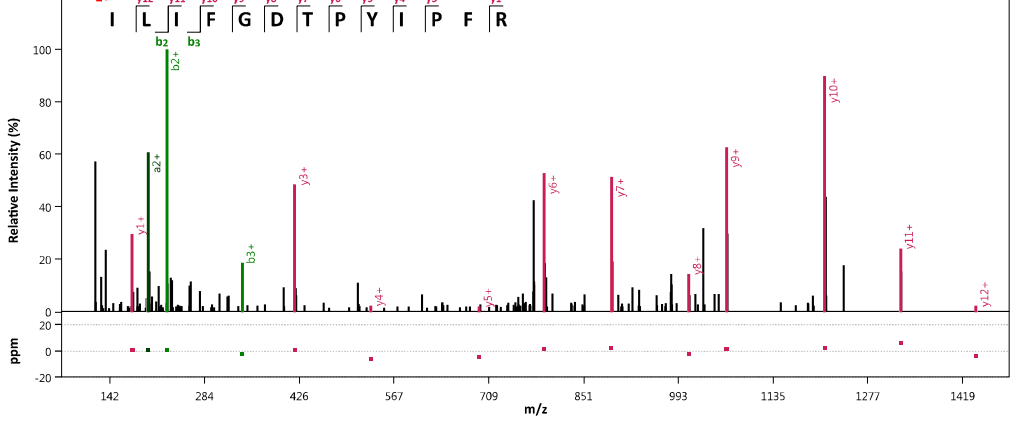

Titte:4817_HF01_XP_CHPP_peptide_Test_5ul_60ms_AGC2E4.40188.40188.2.20.dta Label: None Info:4817__HF01_XP_CHPP_peptide_Test_5Ul_60ms_AGC2E4 Base Peak:5.73E+006 MS2_Mass: 1551.85945Da / 776.433363Th MS2_mass - Theoretical_Mass:0.002542Da / 1.638ppm PSM_Score (\%):66.369

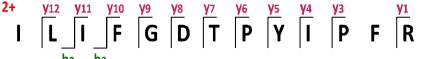
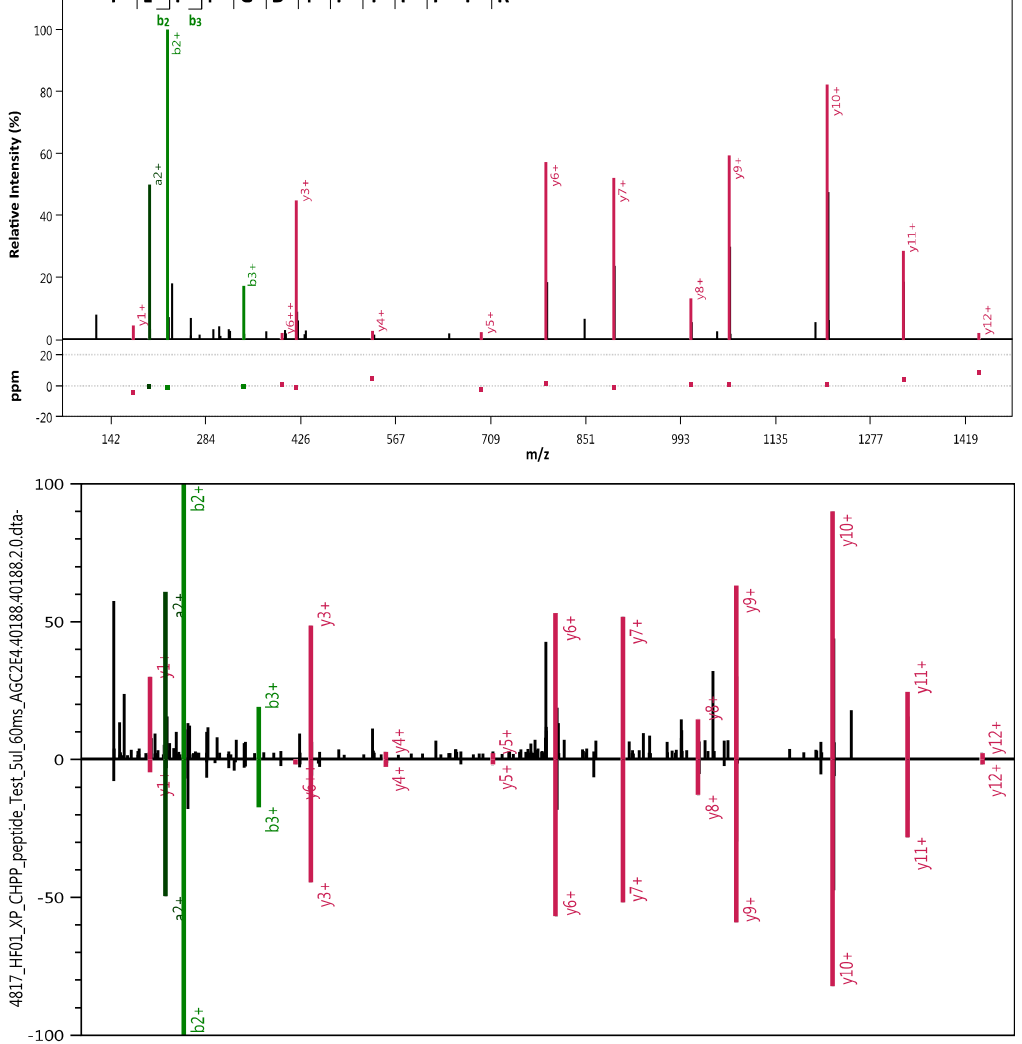
Title: 4817_HF01_XP_CHPP_Has_Tricine10_17_5ul_60ms_AGC2E4.25002.25002.2.0.dta Label: None Info: 4817_HF01_XP_CHPP_Has_Tricine10_17_5UL_60ms_AGC2E4

ass:0.002802Da / 1.816ppm PSM_Score (\%): 38.315

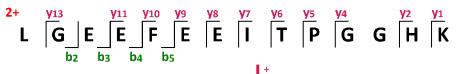

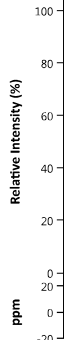

2

름

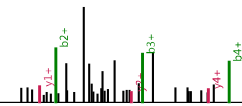
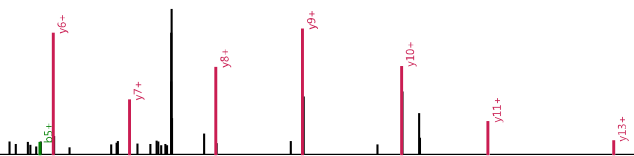

के
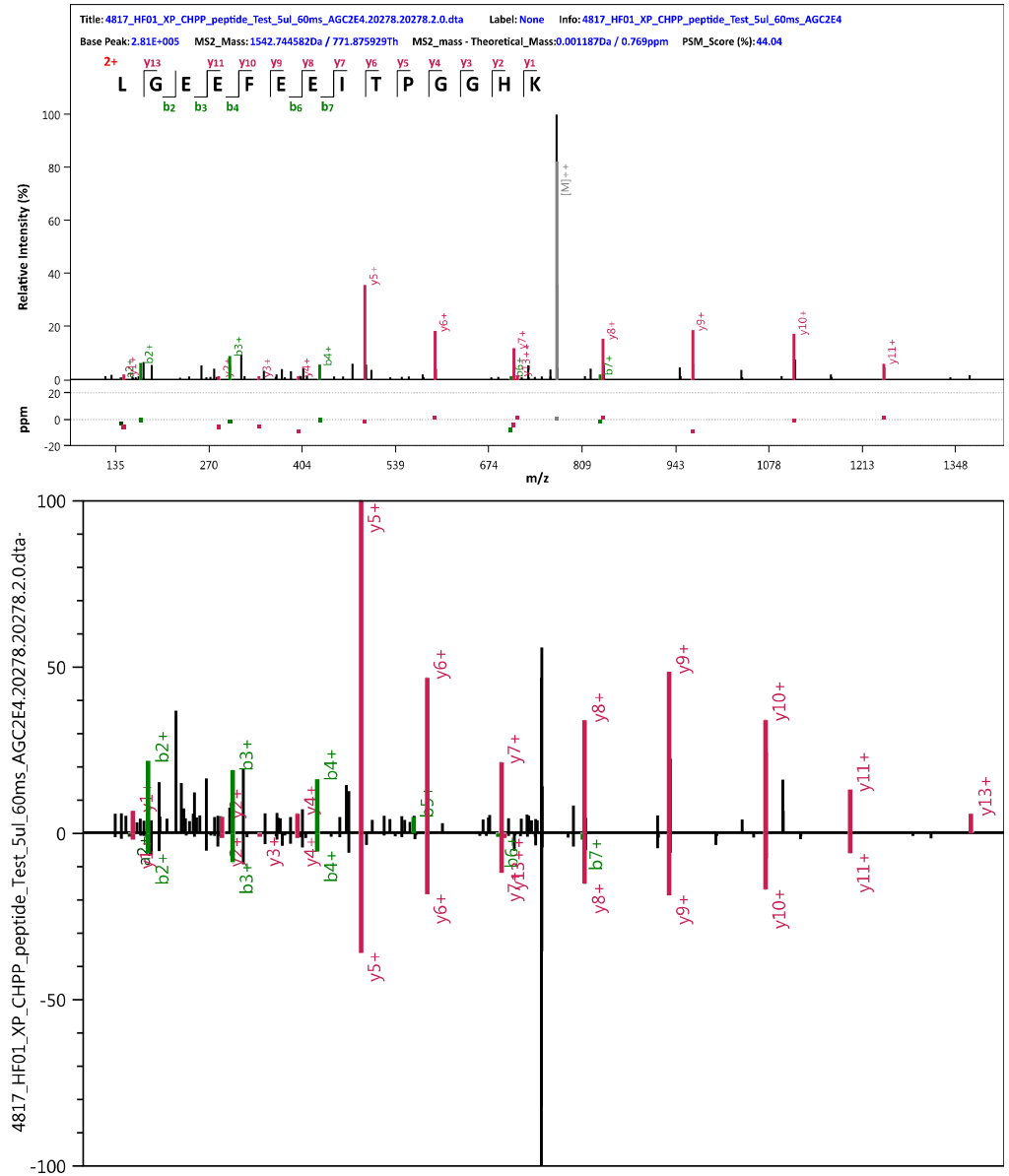


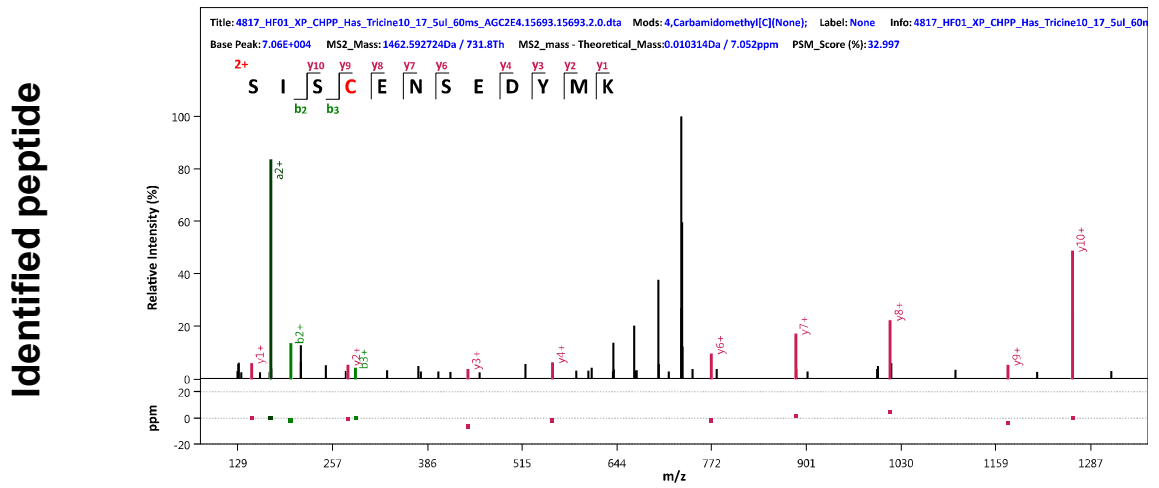

वृ.
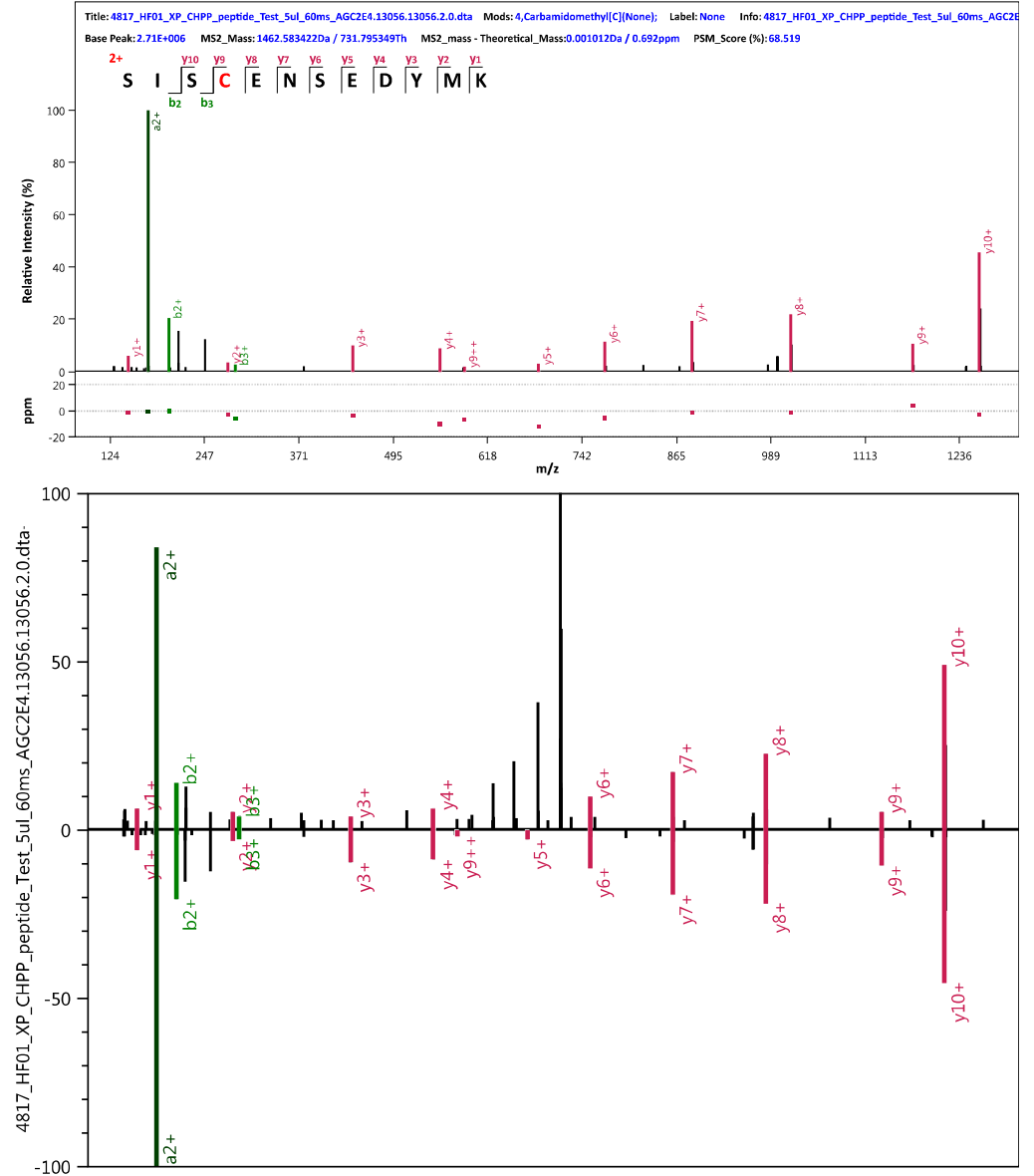

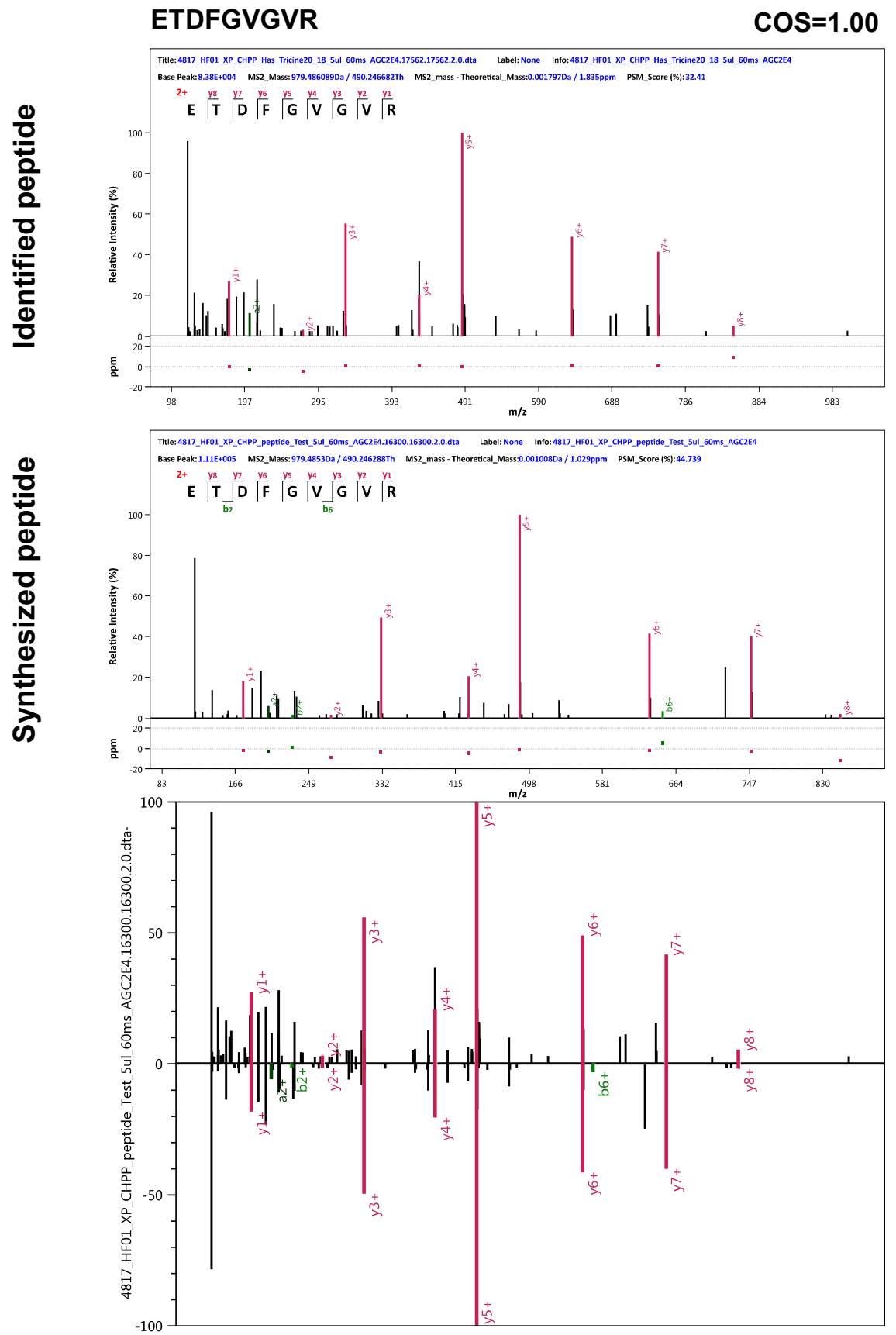

S-13 


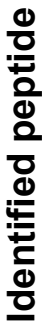

Title: 4817_HF01_XP_CHPP_Has_SDS10_27_6ul_60ms_AGC2E4.37418.37418.3.0.dta Label: None Info: 4817_HF01_XP_CHPP_Has_SDS10_27_6ul_60ms_AGC2E4

Base Peak:6.93E+004 MS2_Mass: 1127.717652Da/376.577401Th MS2_mass - Theoretical_Mass:0.003039Da/2.695ppm PSM_Score (6): 30.096

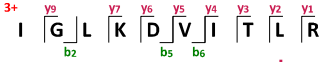

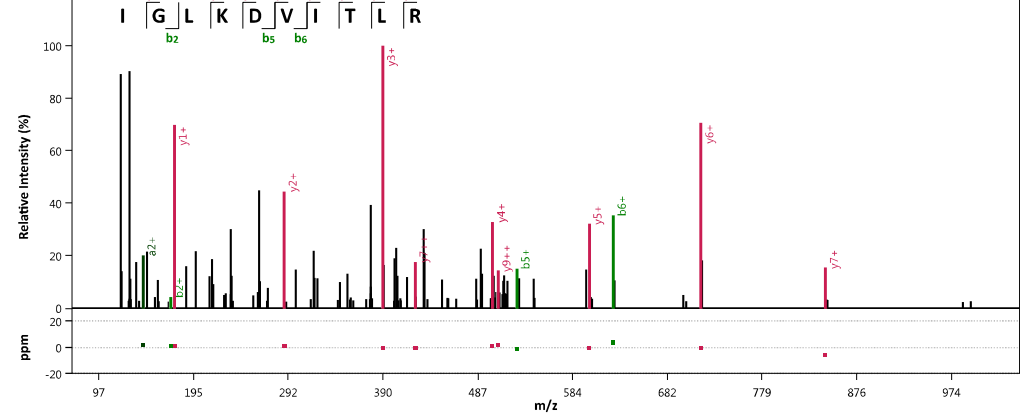

के

Title: 4817_HF01_XP_CHPP_peptide_Test_5ul_60ms_AGC2E4.27730.27730.3.0.dta Label: None Info: 4817_HF01_XP_CHPP_peptide_Test__5ul_60ms_AGC2E4

Base Peak: 1.58E+006 MS2_Mass: 1127.715421Da / 376.576658Th MS2_mass- Theoretical_Mass:0.000809Da/ 0.717ppm PSM_Score (\%): 33.487

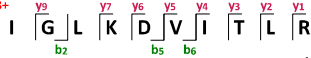
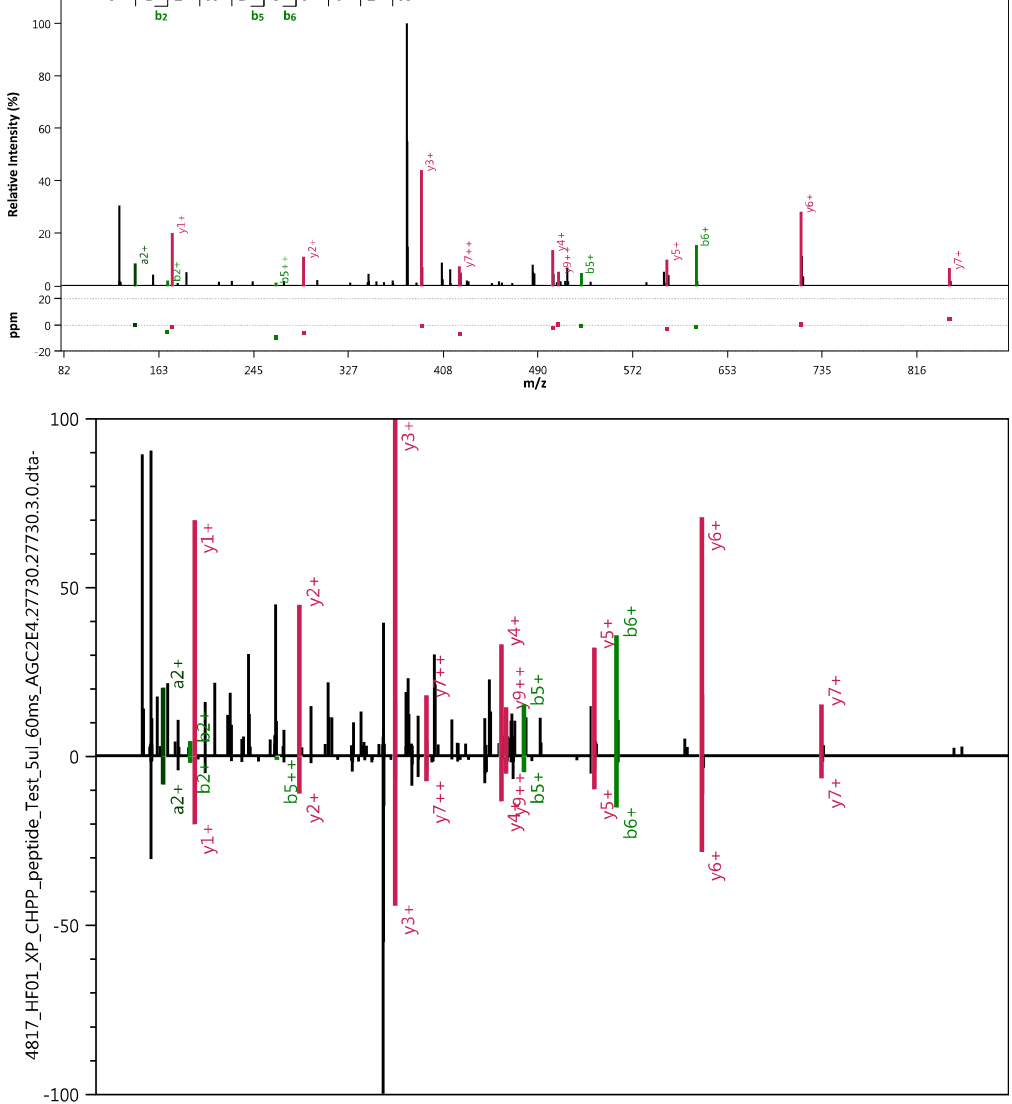
Title: 4817_HF01_XP_CHPP_Has_SDS30_28_5ul_60ms_AGC2E4.39395.39395.2.0.dta Label: None Info: 4817_HF01_XP_CHPP_Has__SDS30_28_5UL_60m__AGC2E4 Base Peak:4.02E+005 MS2_Mass: 1076.648314Da / 538.827795Th MS2_mass - Theoretical_Mass:0.002110Da/1.960ppm PSM_Score (\%): 36.085

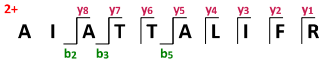

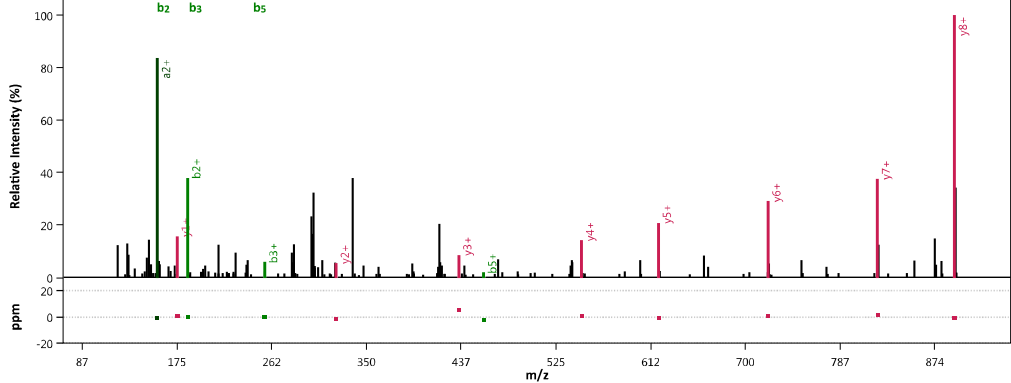

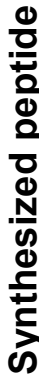

Title: 4817_HF01_XP_CHPP_peptide_Test_5ul_60m5_AGC2E4.28842.28842.2.0.dta Label: None Info: 4817_HF01_XP_CHPP_peptide_Teet_5ul_60ms_AGC2E4 Base Peak: 1.05E+006 MS2_Mass: 1076.648346Da / 538.827811Th MS2_mass - Theoretical_Mass:0.002142Da/1.989ppm PSM_Score (\%):58.238

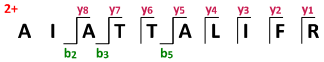
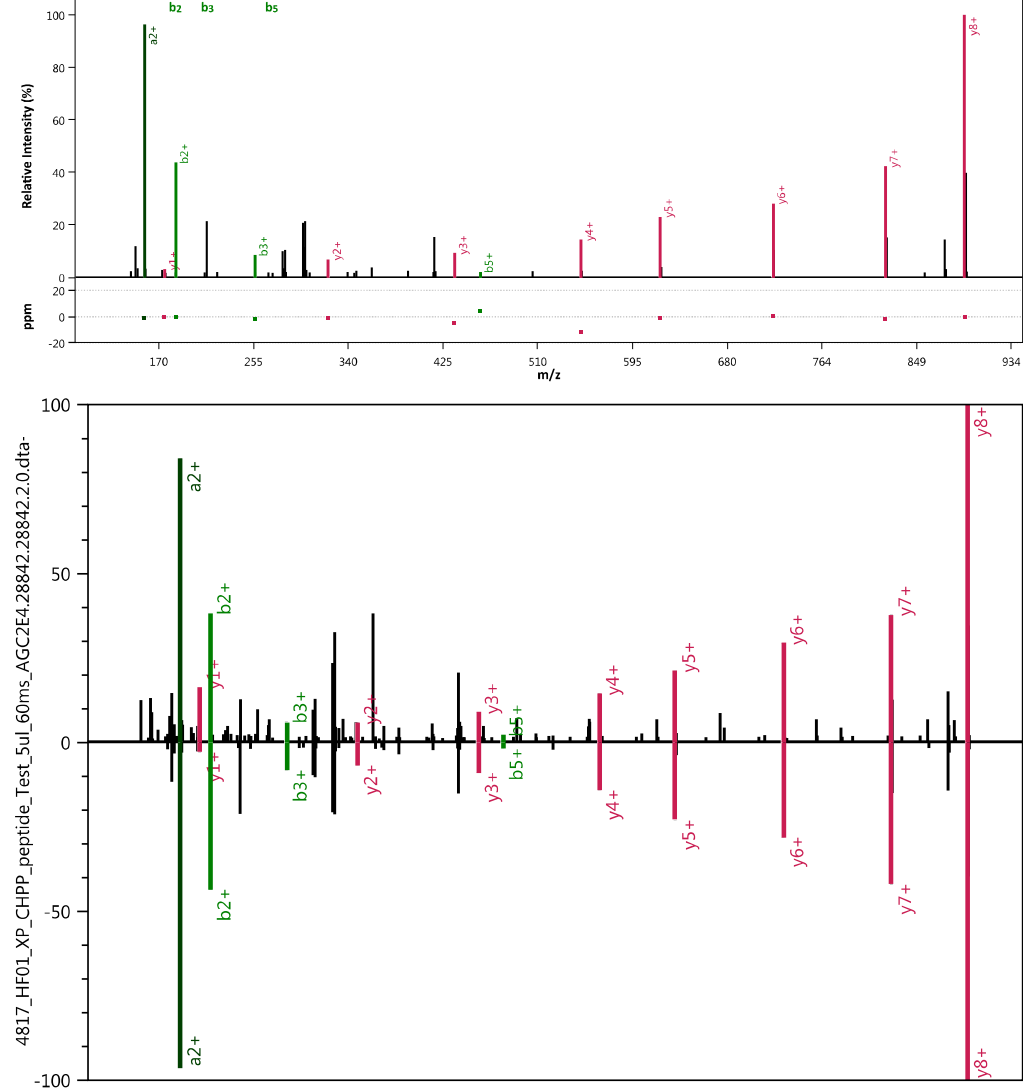


\section{SLHLEGDPNPSAAPTSTCAPR}

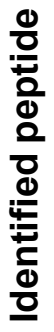

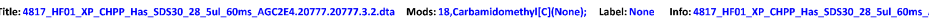
lase Peak:3.99E+005 MS2_Mass:2178.028027Da / 726.68086Th MS2_mass - Theoretical_Mass:0.003719Da / 1.707ppm PSM_Score (\%):34.40

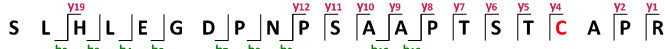

$\frac{0}{0}$
$\frac{0}{0}$
00
$\frac{0}{0}$
$\frac{N}{0}$
$\frac{N}{0}$
$\frac{1}{2}$
क

Title: 4817_HF01_XP_CHPP_peptide_Test_5ul_60ms_AGC2E4.15449.15449.3.0.dta Mods: 18,CGarbamidomethyIICI(None); Label: None Info: 4817_HF01_XP_CHPP_peptide_Test_5ul_60ms_AGC

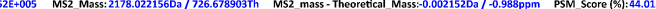

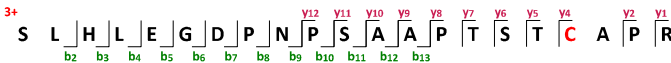
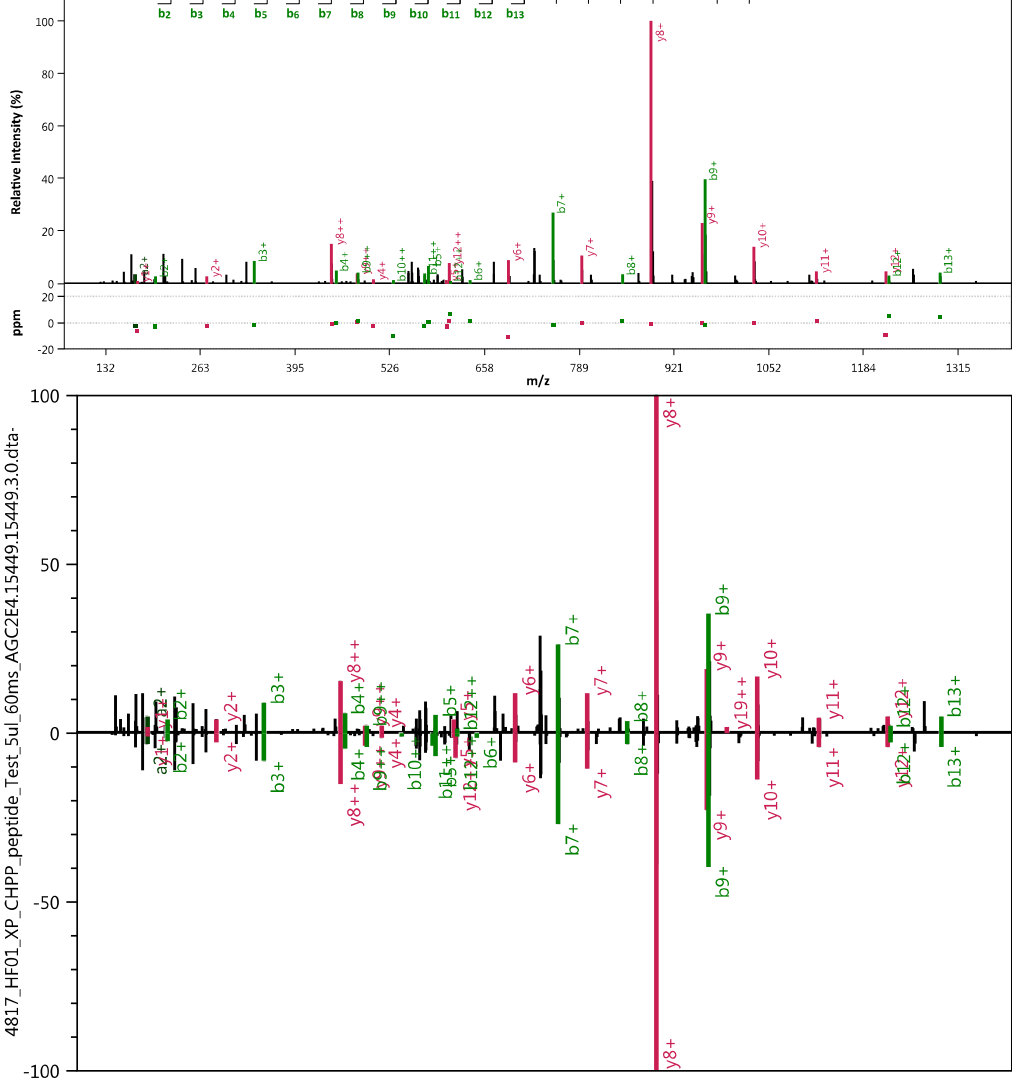
AAEALEAAAAR

$\cos =0.99$
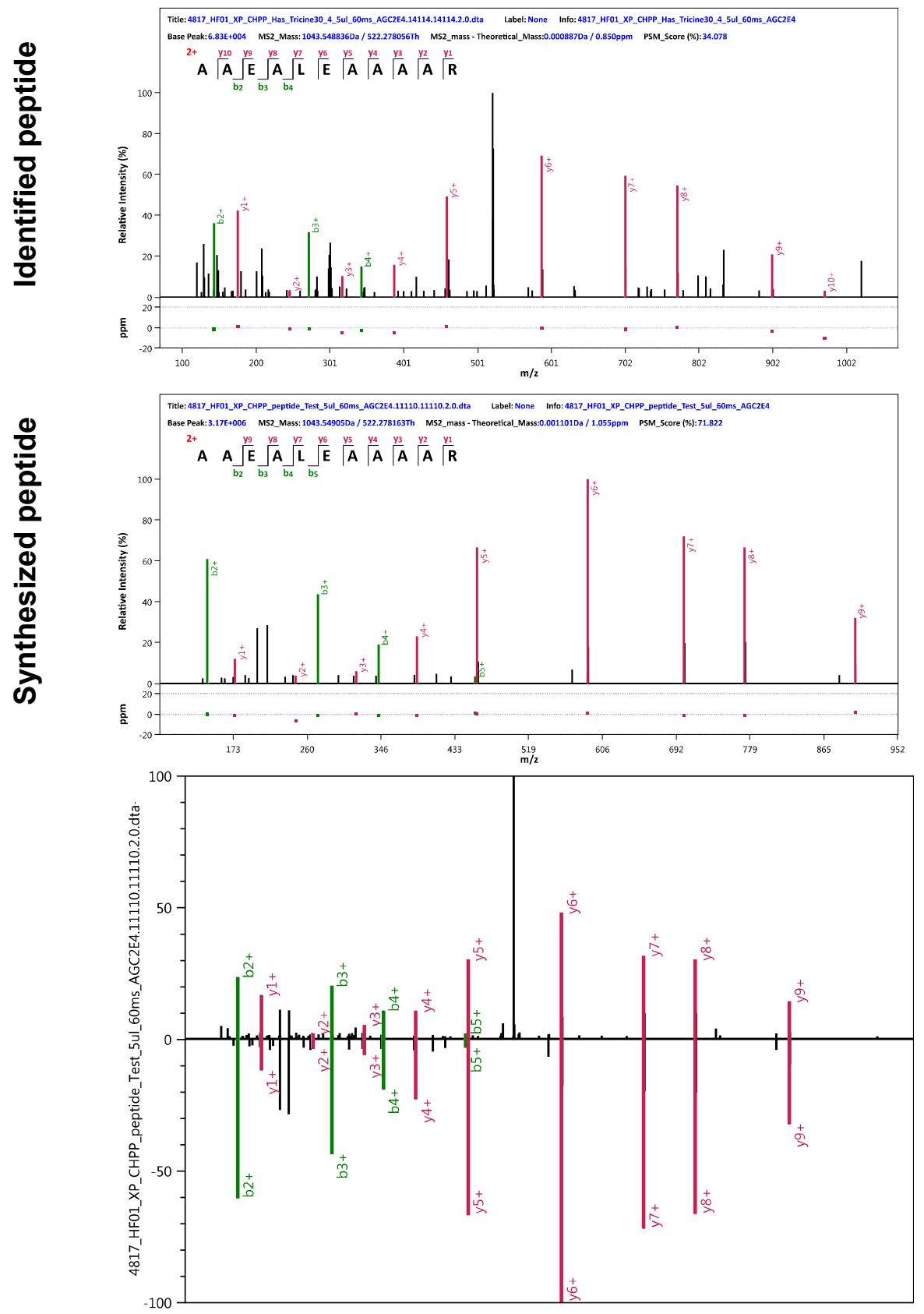

S-17 
Title: 4817_HF01_XP_CHPP_Has__SDS30_9__5ul_60ms_AGC2E4.28129.28129.2.0.dta Label: None Info: 4817_HF01_XP_CHPP_Has__SDS30_9_5ul_60ms_AGC2E4 Base Peak: 1.58E+005 MS2_Mass: 1211.700951Da / 606.354114Th MS2_mass - Theoretical_Mass:0.001596Da/ 1.317ppm PSM_score (\%): 46.119

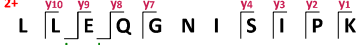

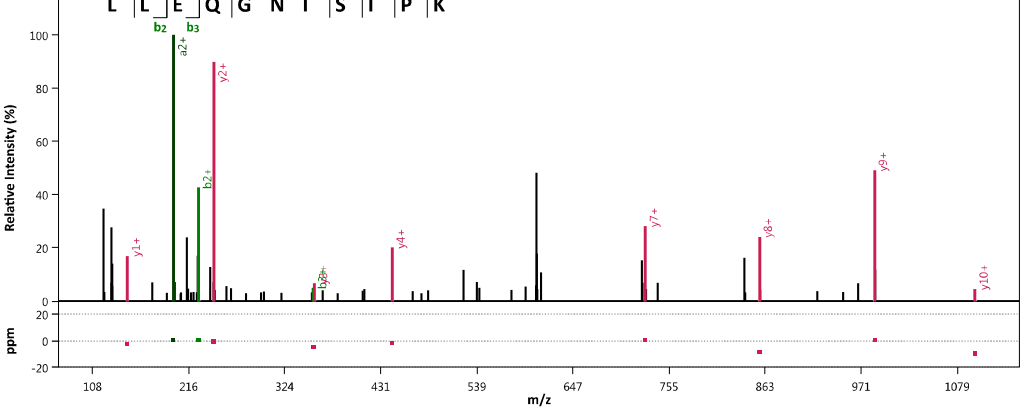

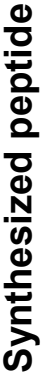

Title: 4817_HF01_XP_CHPP_peptide_Test_5ul_600ms_AGC2E4.21810.21810.2.0.dta Label: None Info: 4817_HF01_XP_CHPP peptide_Test_5ul_60ms_AGC2EA

Base Peak:1.80E+006 MS2_Mass: 1211.701484Da / 606.35438Th MS2_mass - Theoretical_Mass:0.002129Da/ 1.757ppm PSM_Score (\%): 67.314

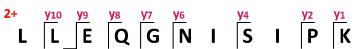
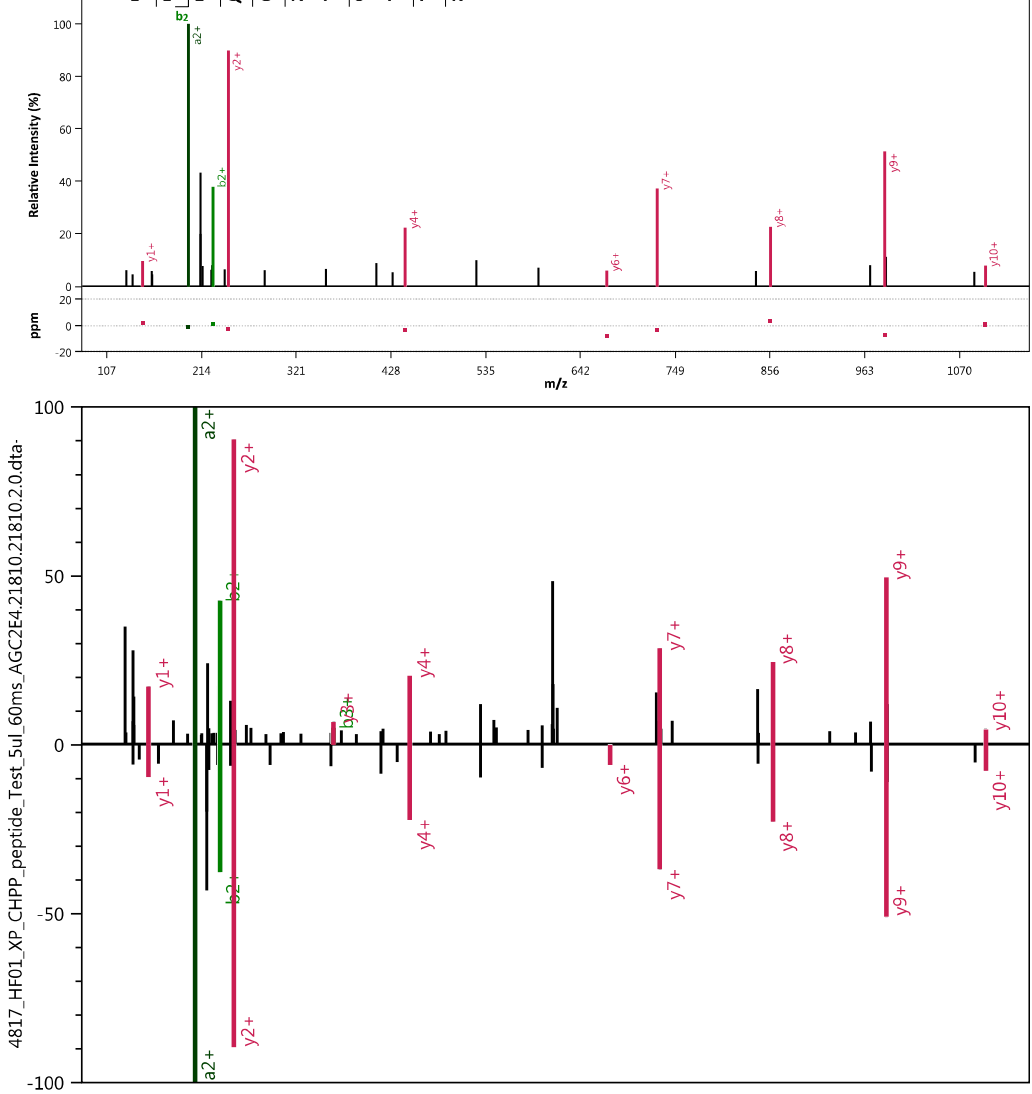


\section{ALQDEQESQR}

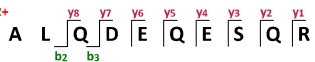

क्ष
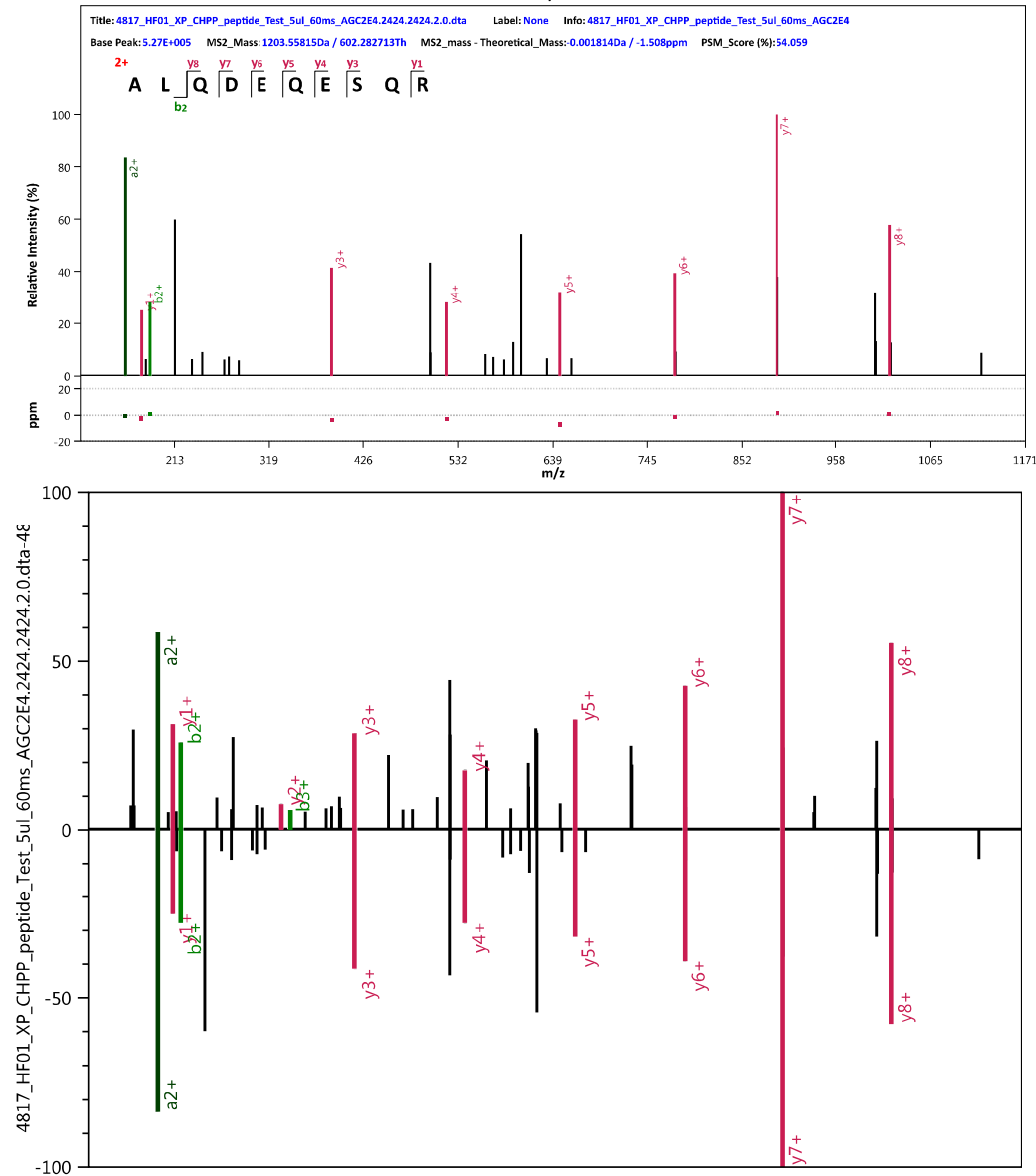


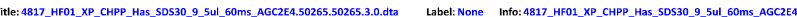
Base Peak: 3.15E+004 MS2_Mass: 1845.913966Da / 615.976173Th MS2_mass - Theoretical_Mass:0.008925Da/4.835ppm PSM__core (\%):42.548

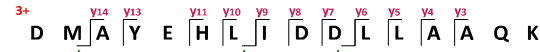

ํㅗㅇ

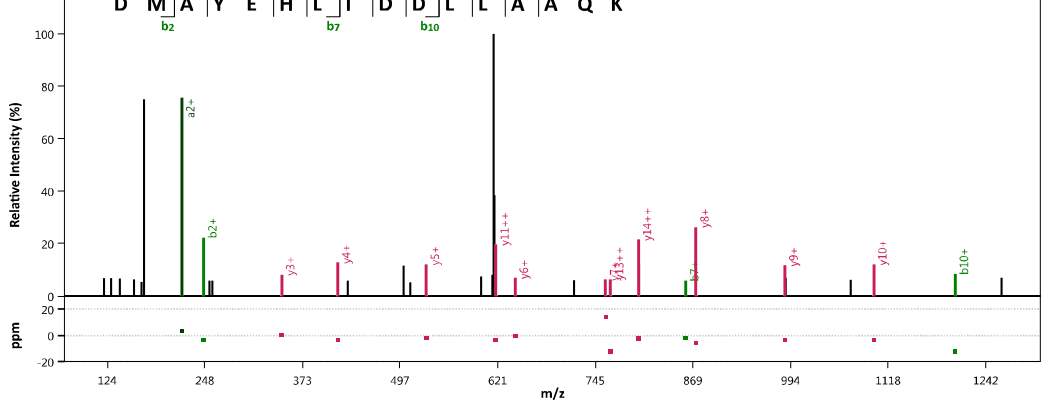

Title: 4817_HF01_XP_CHPP_peptide_Test_5ul_60m5_AGC2E4.40650.40650.3.0.dta Label: None Info: 4817_HF01_XP_CHPP_peptide_Test_5ul_60ms_AGC2E4

Base Peak:2.08E+005 MS2_Mass: 1845.909925Da / 615.974826Th MS2_mass - Theoretical_Mass:0.004884Da / 2.646ppm PSM_Score (\%): 45.504

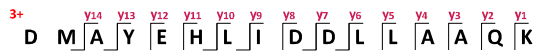
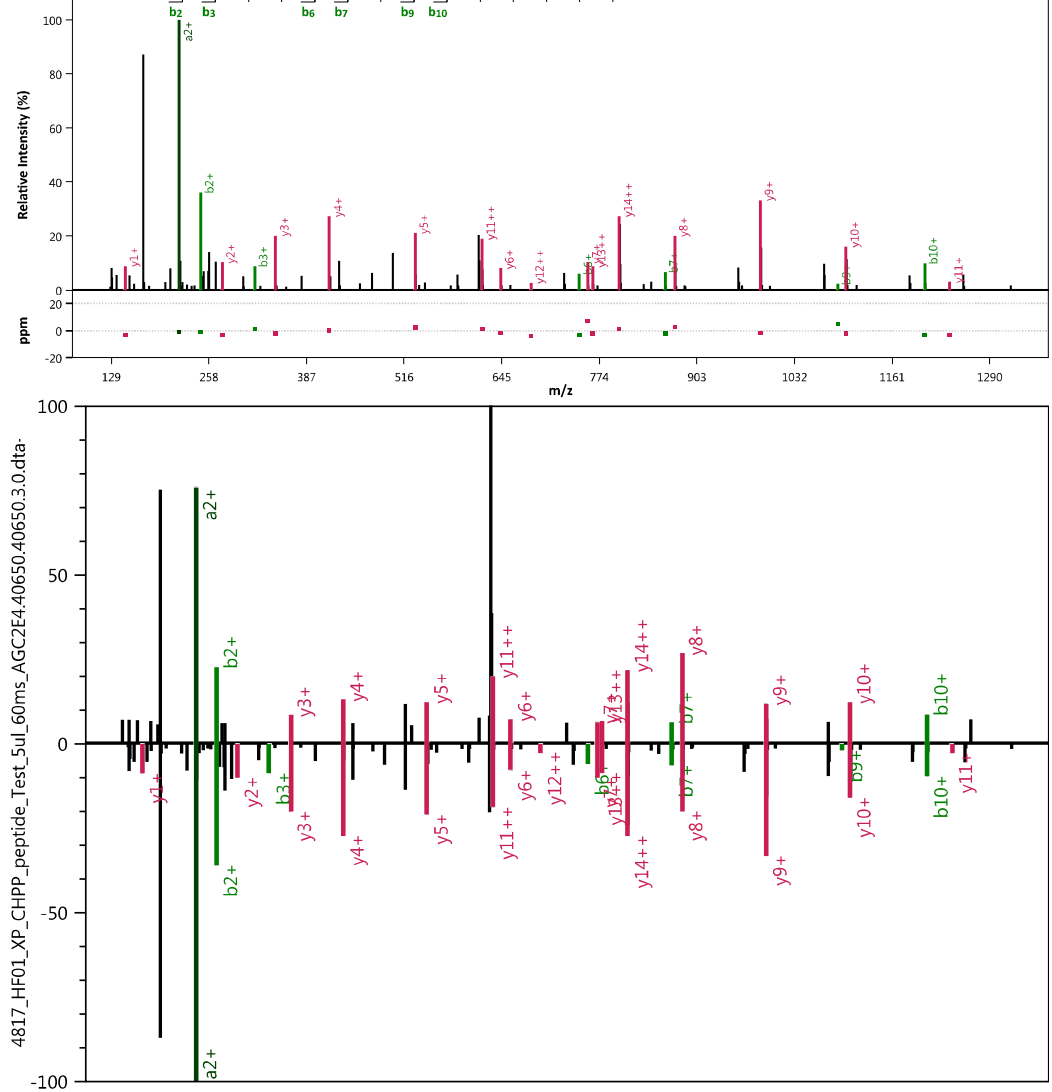


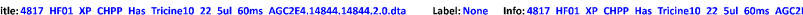
Base Peak: 3.36E+004 MS2_Mass: 1205.558007Da / 603.282642Th MS2_mass - Theoretical_Mass:0.003520Da / 2.920ppm PSM_Score (\%): 38.957

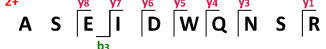

कृ
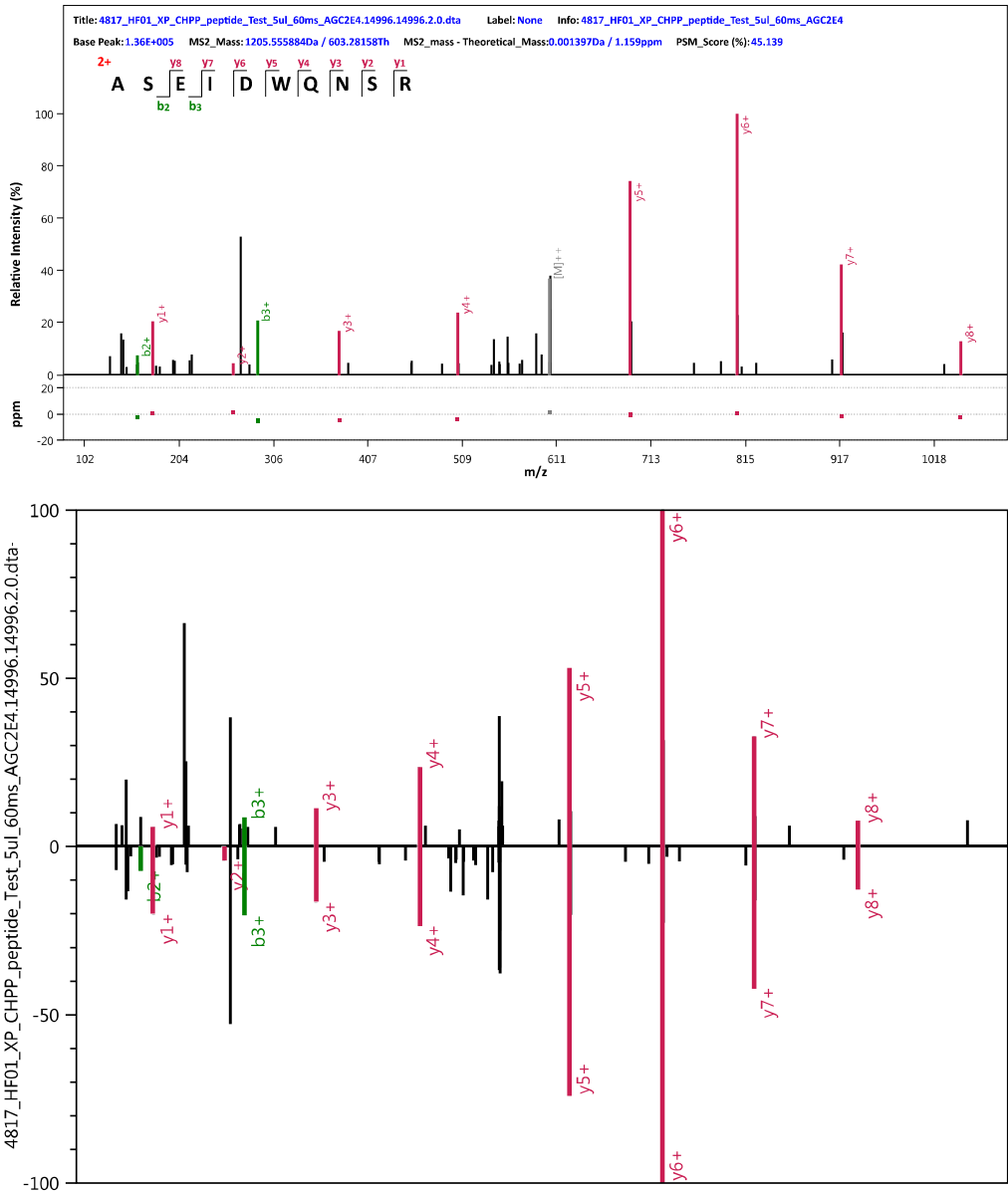


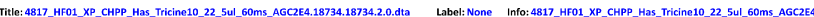
Base Peak: 3.89E+004 MS2_Mass: 1146.620943Da / 573.81411Th MS2_mass - Theoretical_Mass:0.002277Da/ 1.986ppm PSM_Score (\%): 52.184

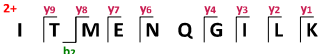

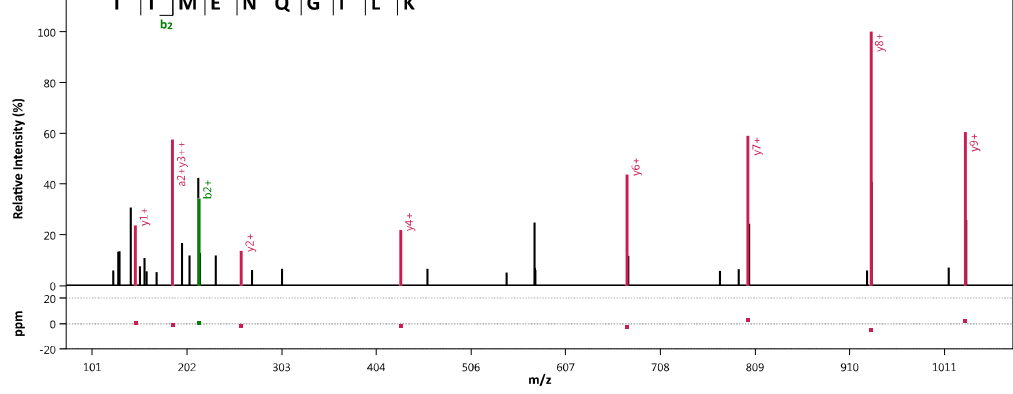

을

Title: 4817_HF01_XP_CHPP_peptide_Test_5ul_60ms_AGC2E4.18486.18486.2.0.dta Label: None Info: 4817_HF01_XP_CHPP_peptide_Test_5ul_60ms_AGC2E4

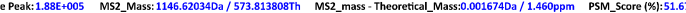

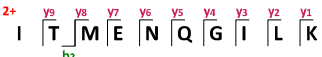
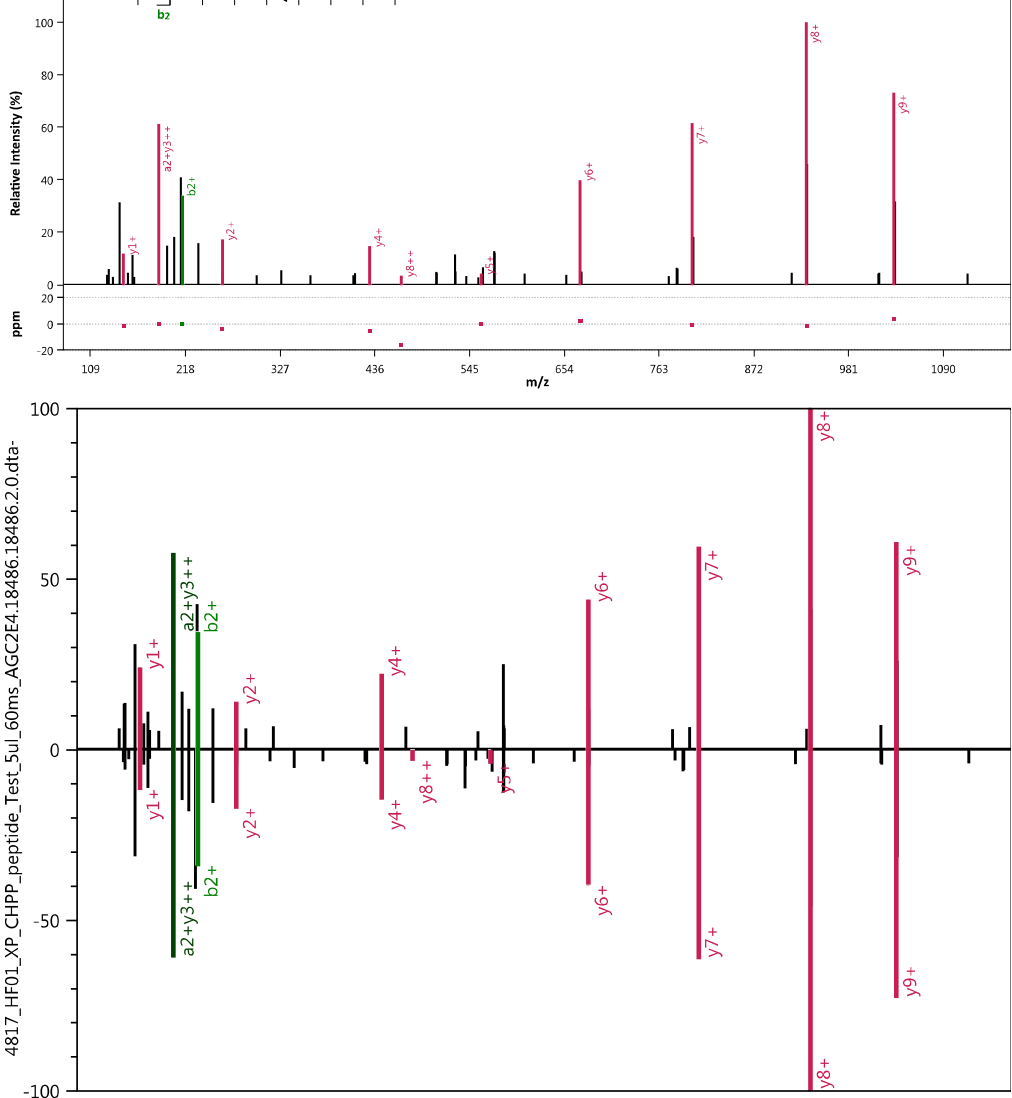
Title: 4817_HF01_XP_CHPP_Has__Tricine20_15_5ul_60ms_AGC2E4.6031.6031.2.0.dta Label: None Info: 4817_HF01_XP_CHPP_Has__Tricine20_15_5ul_60ms_AGC2E4

PS2_Mass: 1059.559436Da / 530.283356Th MS2_mass - Theoretical_Mass:0.001316Da/1.242ppm PSM_Score (\%):56.705

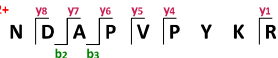

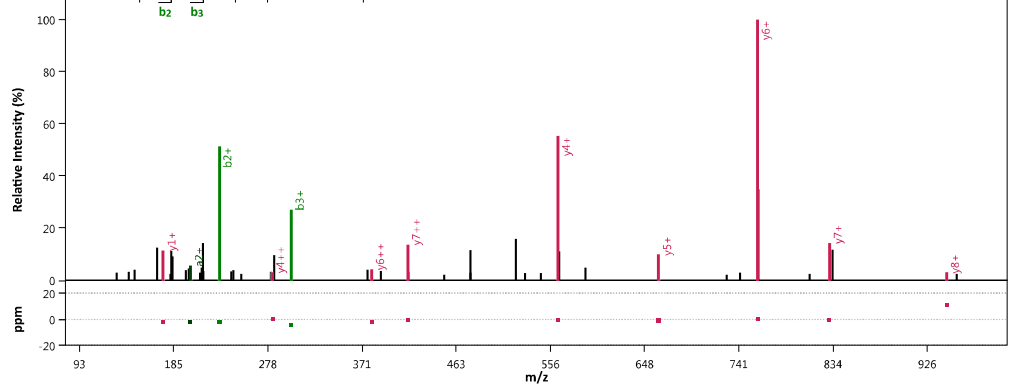

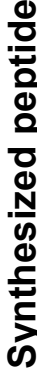

TItle: 4817 HF01_XP_CHPP_peptide Test__Sul_60ms__AGC2E4.4653.4653.2.0.dta Label: None Info: 4817_HF01_XP_CHPP_peptide_Test_5ul_60ms_AGC2E4 Base Peak: 3.42E+006 MS2_Mass: 1059.55823Da/ 530.282753Th MS2_mass - Theoretical_Mass:0.000109Da / 0.103ppm PSM_Score (*): 70.22

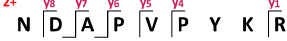
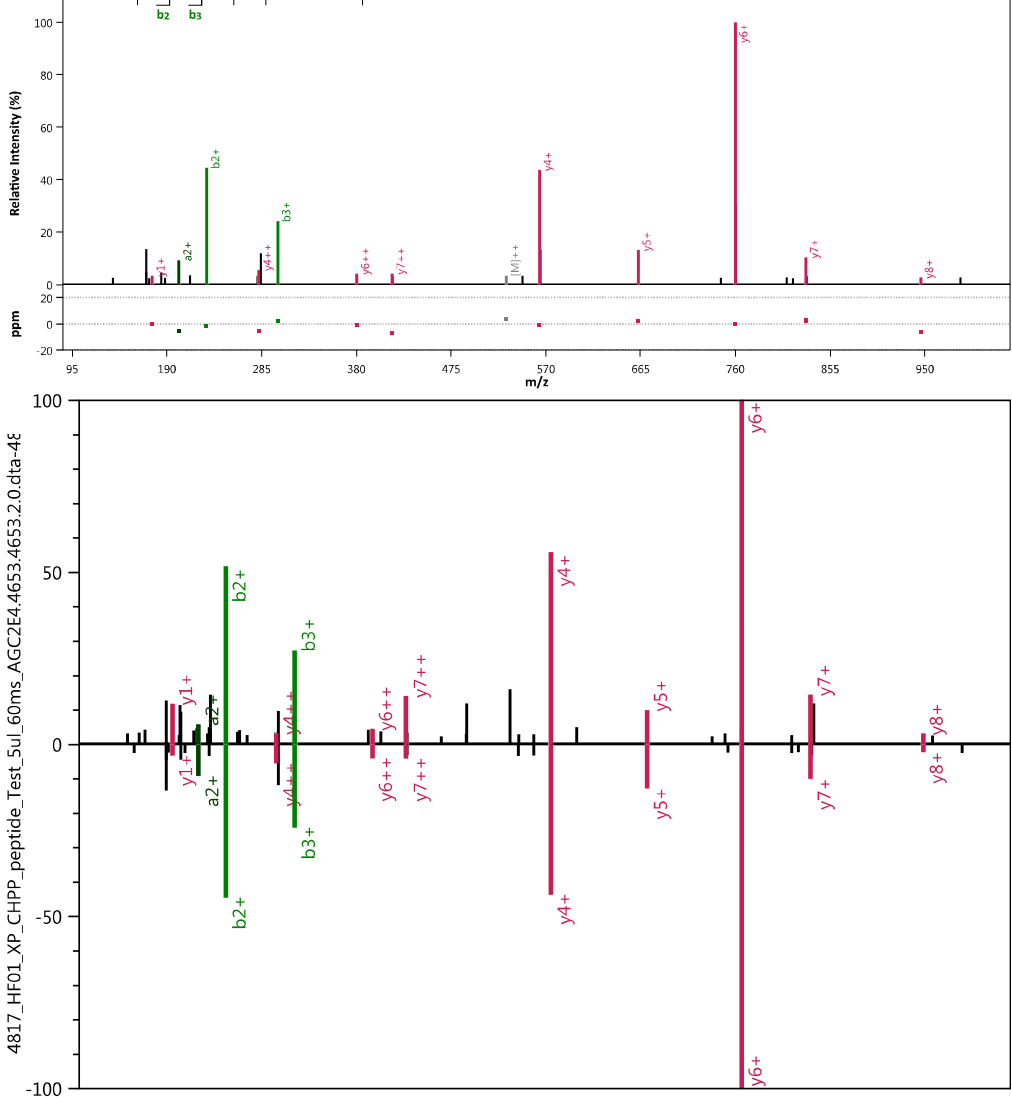


\section{SLQELANSEAVQFLR}

The:481__HF01_XP_CHPP_Has_SDS30_27_Sul_60ms_AGC2E4.37891.37891.2.0.dta Label: None Info: 4817_HF01_XP_CHPP_Has_SDS30_27_5U1_60m5_AGC2E4

Peak: 6.82E+004 MS2_Mass: 1704.892106Da/852.949691Th MS2_mass- Theoretical_Mass:0.000662Da/0.388ppm PSM_Score (\%): 59.57

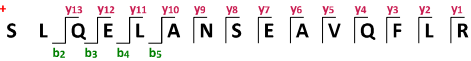

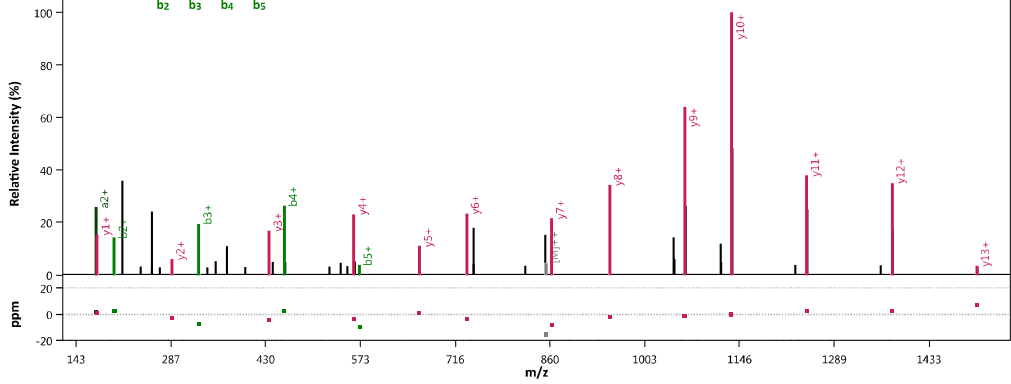

के

Title: 4817_HF01_XP_CHPP_peptide_Test_5ul_60ms_AGC2E4.35779.35779.2.0.dta Label: None Info: 4817_HF01_XP_CHPP_peptide_Test_5ul_60ms_AGC2E4 Base Peak: 3.27E+006 MS2_Mass: 1704.892692Da/ 852.949984Th MS2_mass - Theoretical_Mass:0.001247Da / 0.732ppm PSM_Score (\%): 60.184

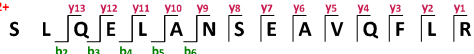
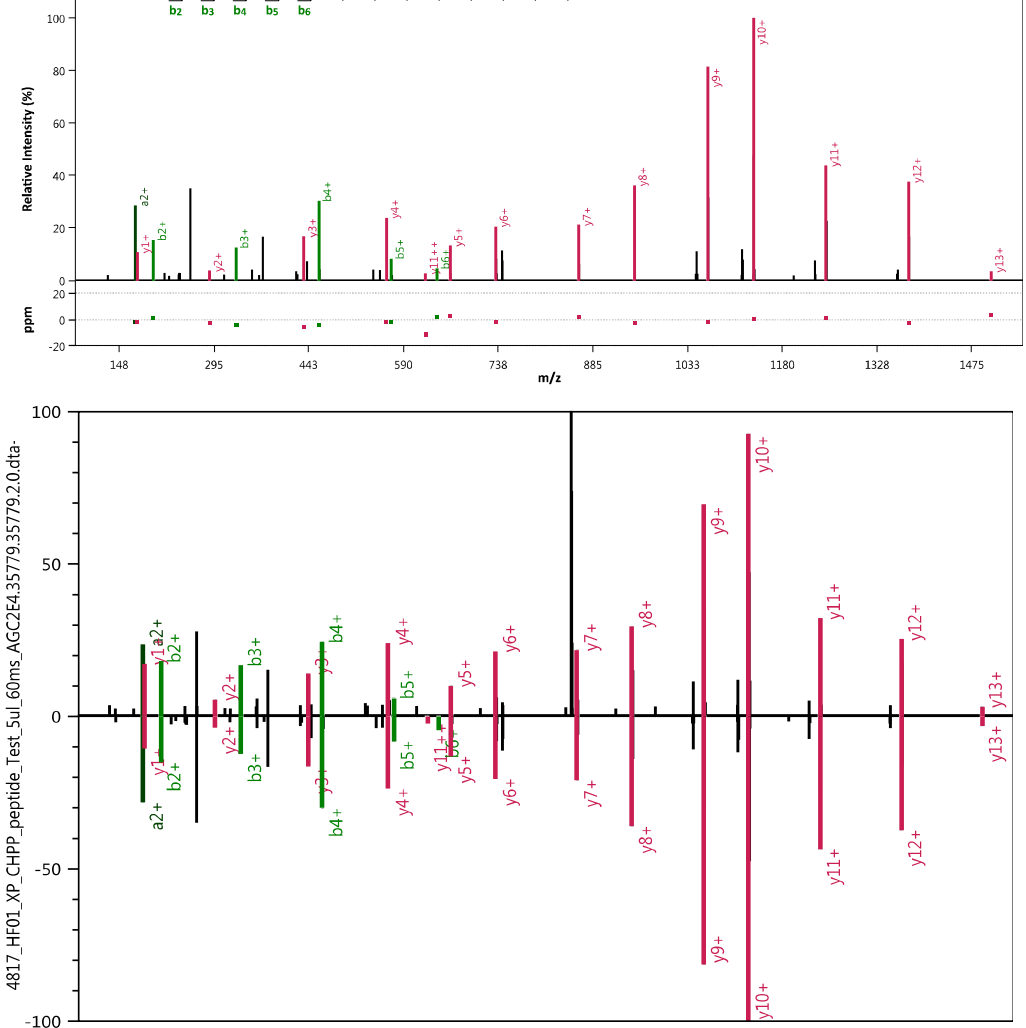

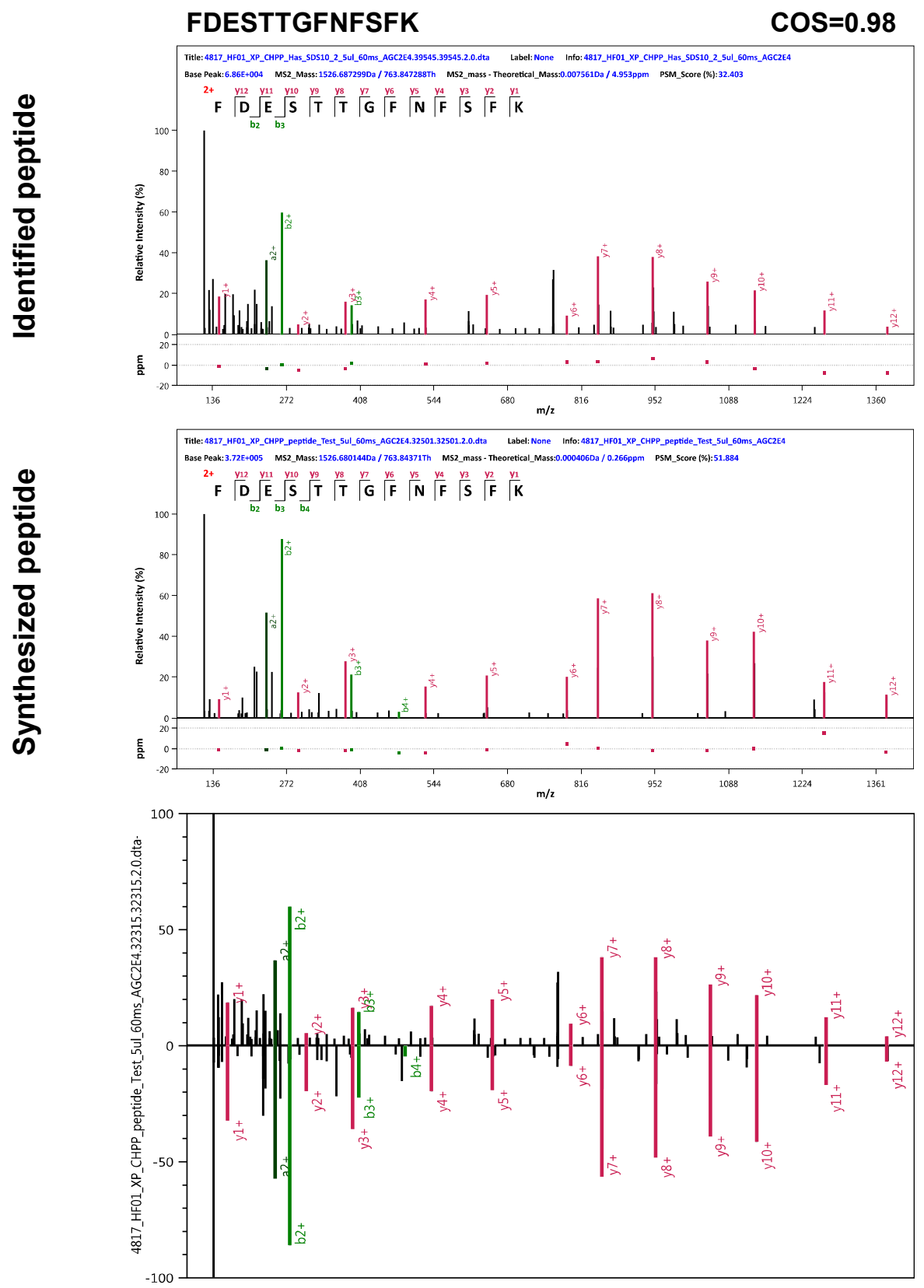

S-25 
Title: 4817_HF01_XP_CHPP_Has_SDS10_2_5ul_60ms_AGC2E4.21913.21913.2.0.dta Label: None Info: 4817_HF01_XP_CHPP_Has_SOS10_2_5U1_60ms__AGC2E4

Base Peak:5.23E+004 MS2_Mass: 1629.755598Da / 815.381437Th MS2_mass - Theoretical_Mass:0.005331Da/ 3.271ppm PSM_Score (\%): 50.983

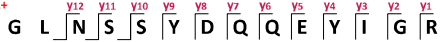

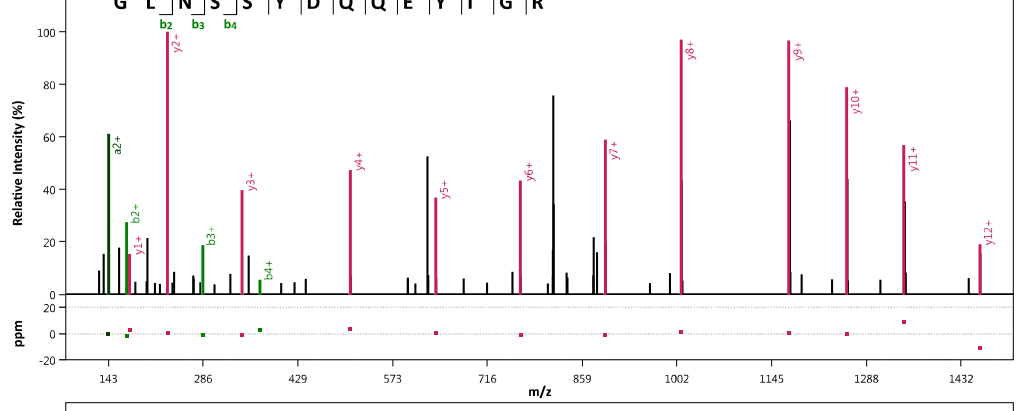

Title: 4817_HF01_XP_CHPP_peptide_Test_5ul_60ms_AGC2E4.17540.17540.2.0.dta Label: None Info: 4817_HF01_XP_CHPP_peptide_Test_5Ul_60ms_AGC2E4

Base Peak: 1.19E+005 MS2_Mass: 1629.75221Da / 815.379743Th MS2_mass - Theorettical_Mass:0.001943Da / 1.192ppm PSM_Score (ж): 60.019

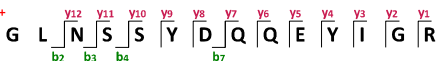

$\frac{0}{\frac{0}{+}}$
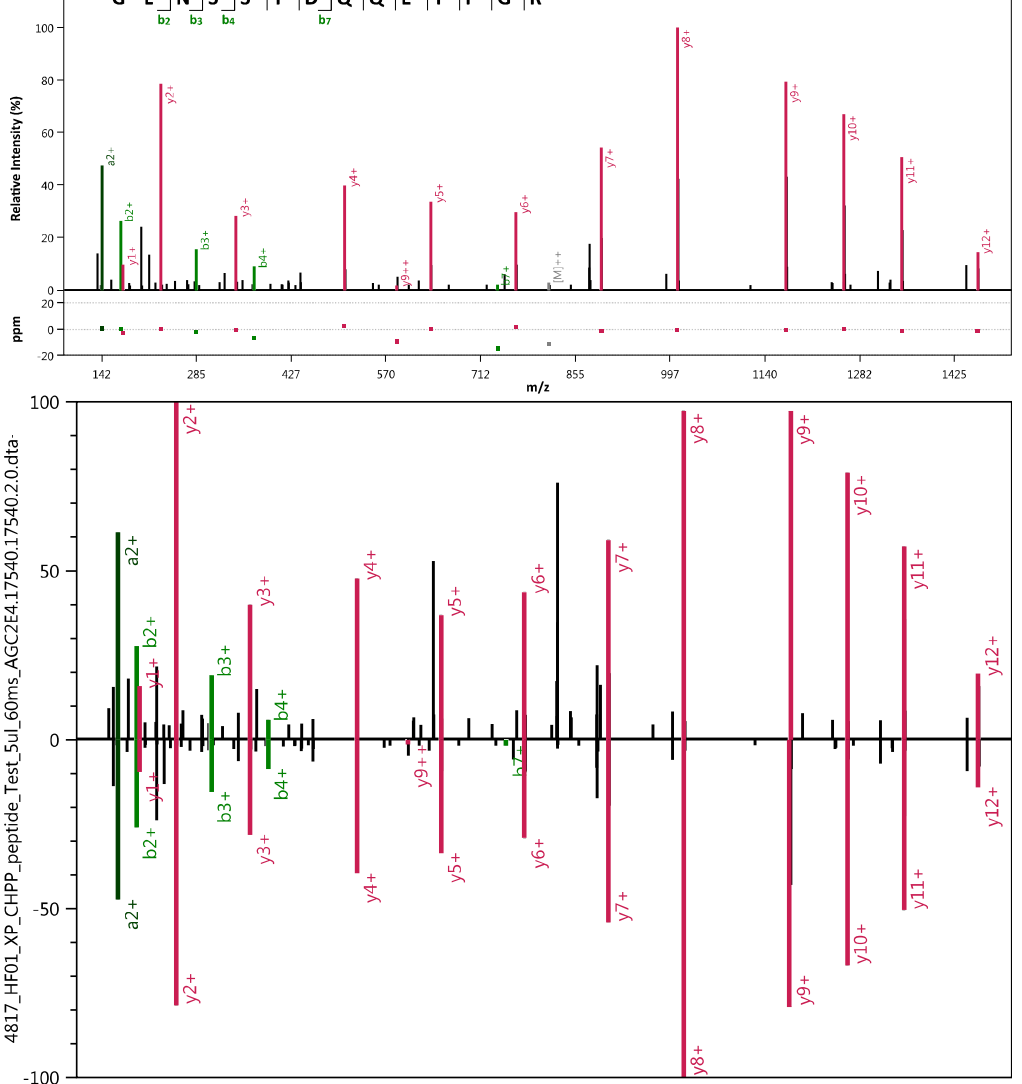


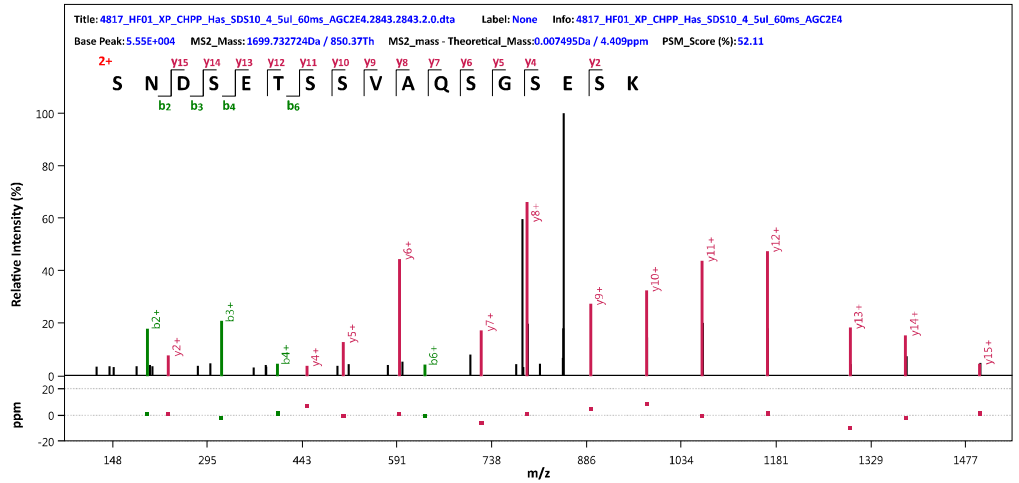

送

Title: 4817_HF01_XP_CHPP_peptide_Test_5ul_60ms_AGC2E4.2325.2325.2.0.dta Label: None Info: 4817_HF01_XP_CHPP_peptide_Test_5ul_60ms_AGC2E4

Base Peak:5.24E+004 MS2_Mass:1699.724496Da / 850.365886Th MS2_mass - Theoretical_Mass:0.000733Da /-0.431ppm PSM_Score (\%):70.097

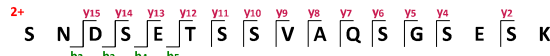
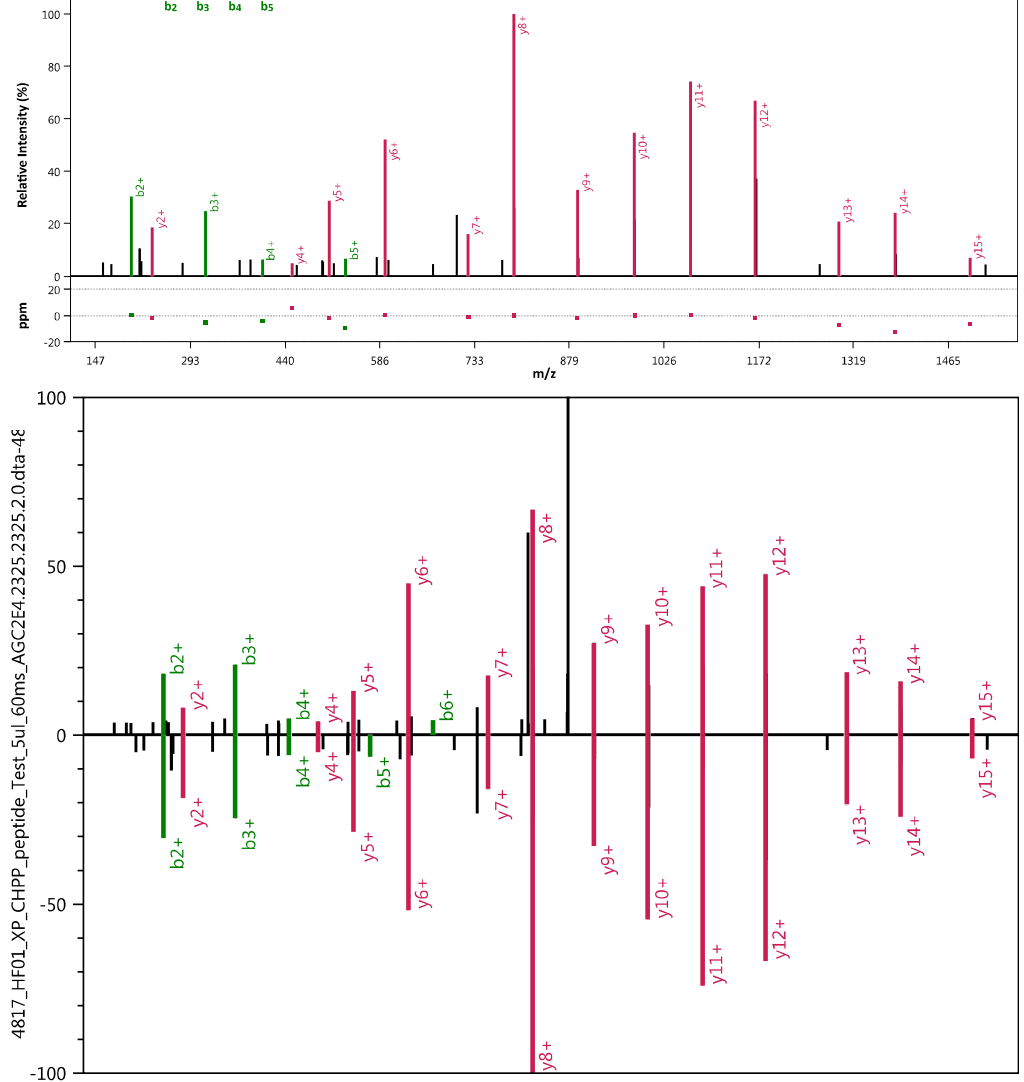


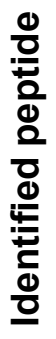

Title: 4817_HF01_XP_CHPP_Has_SDS10_25_SUl_60ms__AGC2E4.39714.39714.20.dta Label: None Info: 4817_HF01_XP_CHPP_Has_SDS10_25_5ul_60ms_AGC2E4 Base Peak: 3.30E+004 MS2_Mass: 1550.7896120a/ 775.898444Th MS2_mass - Theoretical_Mass:0.004748Da / 3.061ppm PSM_Score (\%): 44.878

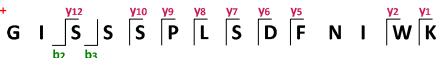

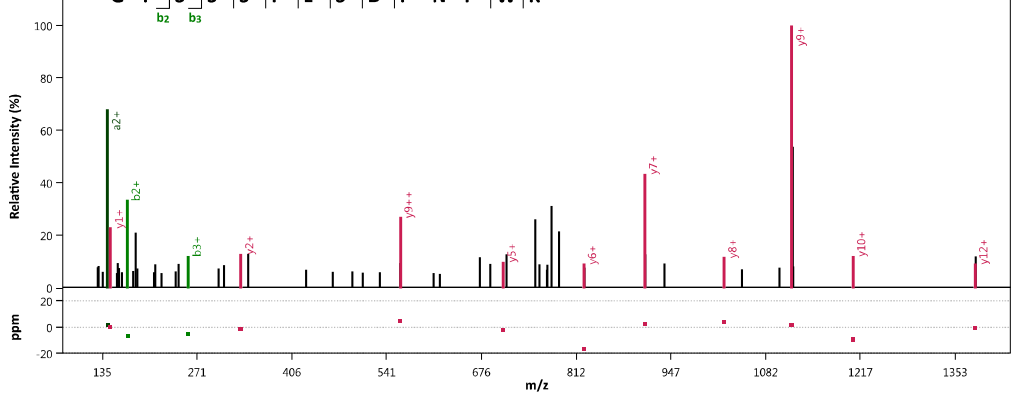

Title: 4817_HF01_XP_CHPP_peptide_Test_5ul_600ms_AGC2E4.37564.37564.2.0.dta Label: None Info: 4817_HF01_XP_CHPP_peptide_Test_5ul_60ms_AGC2E4 Base Peak:7.28E+005 MS2_Mass: 1550.78708Da / 775.897178Th MS2_mass - Theoretical_Mass:0.002215Da / 1.428ppm PSM_Score (x):56.83

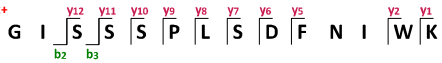
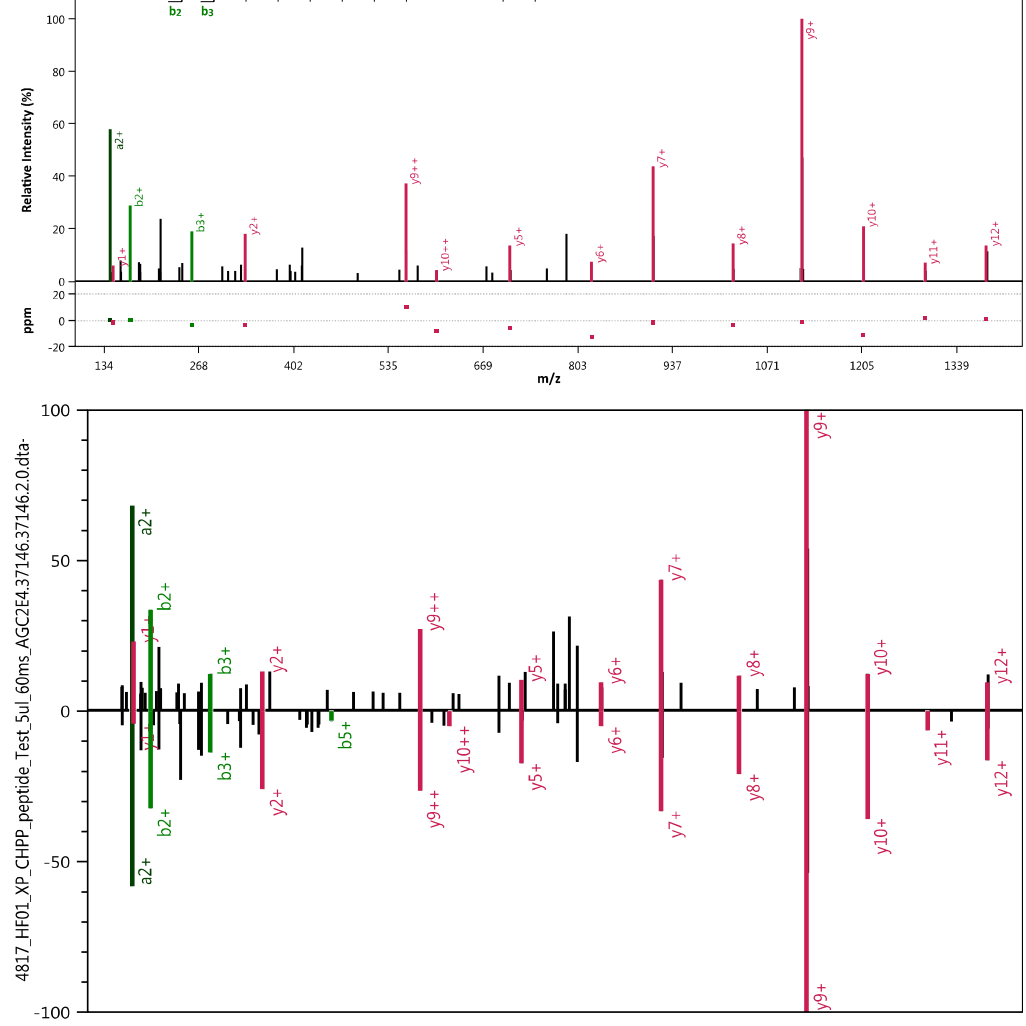


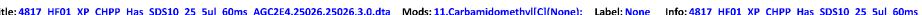
Base Peak: 7.75E+004 MS2_Mass: 1853.905447Da / 618.64Th MS2_mass - Theoretical_Mas5:-0.000658Da / -0.355ppm PSM_Score (\%) : 32.108

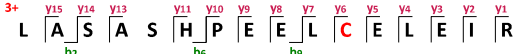

$\frac{0}{0}$
$\frac{0}{0}$
$\frac{0}{0}$
$\frac{0}{0}$
$\frac{N}{0}$
के
के

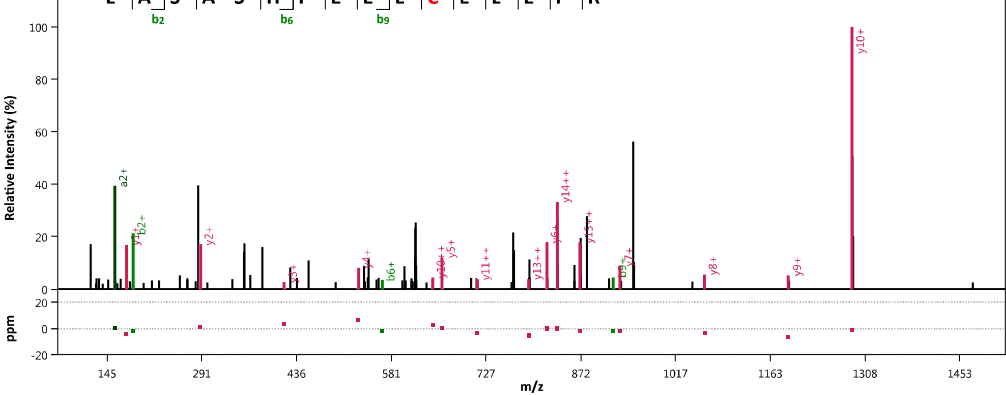

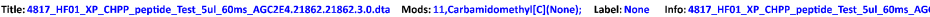

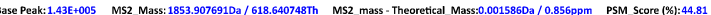

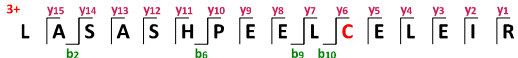
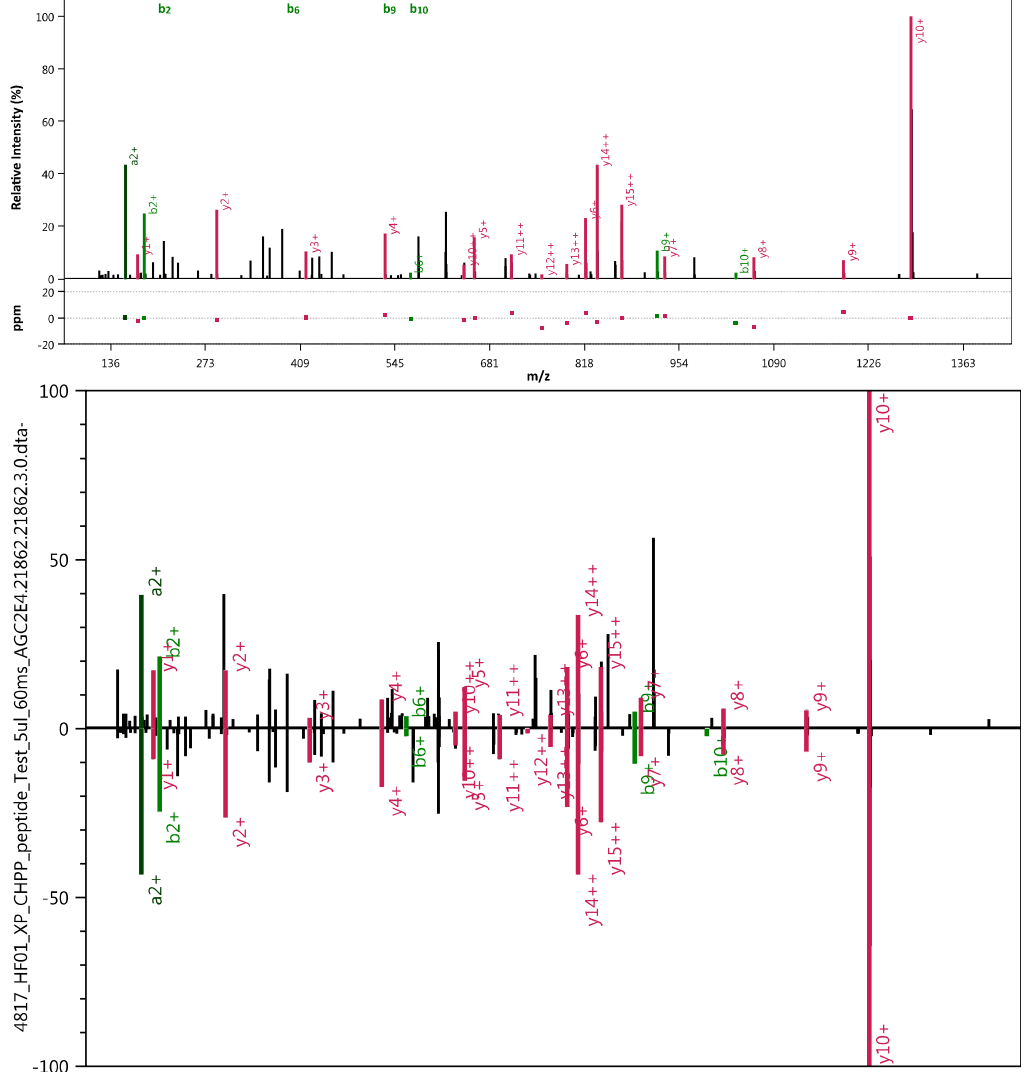


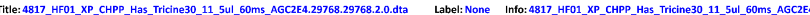

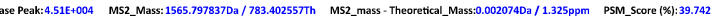

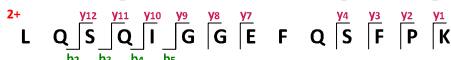

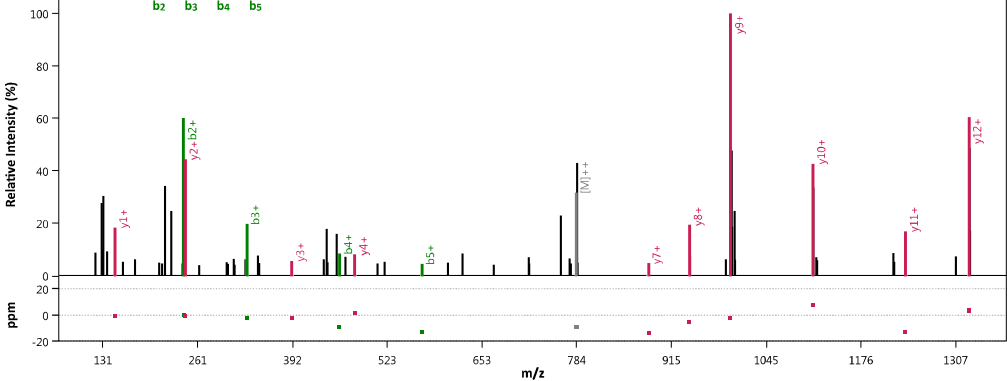

के

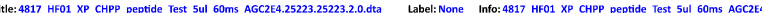
Base Peak: 1.88E+006 MS2_Mass: 1565.796472Da / 783.401874Th MS2_mass - Theoretical_Mass:0.000709Da / 0.453ppm PSM_Score (\%):58.345

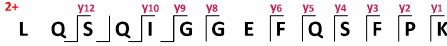
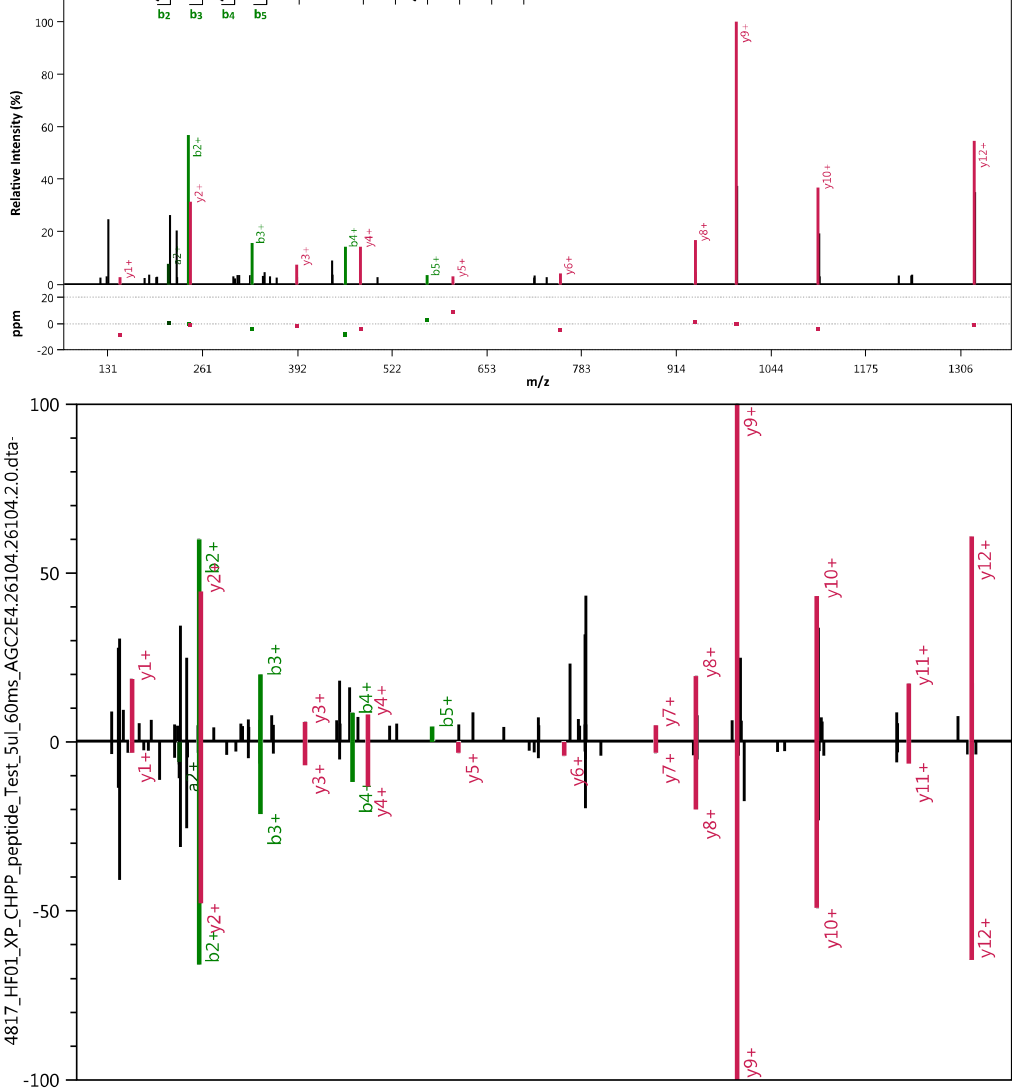


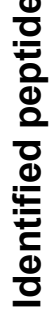

Title: 4817_HF01_XP_CHPP_Has_SDS10_23_Sul_60ms_AGC2E4.46009.46009.2.0.dta Label: None Info: 4817_HF01_XP_CHPP_Has_SDS10_23_5ul_60ms_AGC2E4

Peak:8.18E+004 MS2_Mass: 1336.744332Da/668.875804Th MS2_mass - Theoretical_Mass:0.007198Da/5.385ppm PSM Score (\%): 49.815

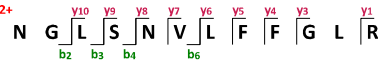

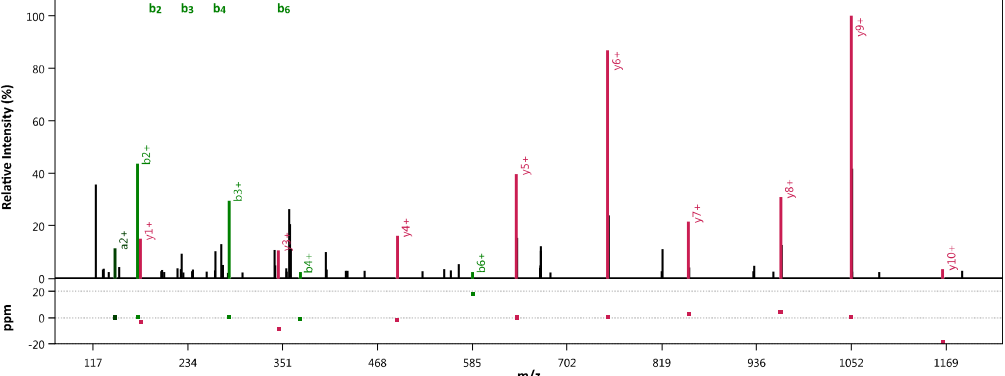

Titte: 4817_HF01_XP_CHPP_peptide_Test_Sul_60ms_AGC2E4.39578.39578.2.0.dta Label: None Info: 4817_HF01_XP_CHPP_peptide_Test_5ul_600ms_AGC2E4

Base Peak:4.20E+006 MS2_Mass: 1336.7389840a / 668.87313Th MS2_mass - Theoretical_Mass:0.001849Da / 1.384ppm PSM_Score (જ): 57.136

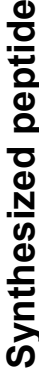

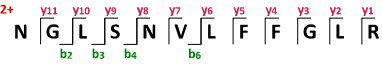
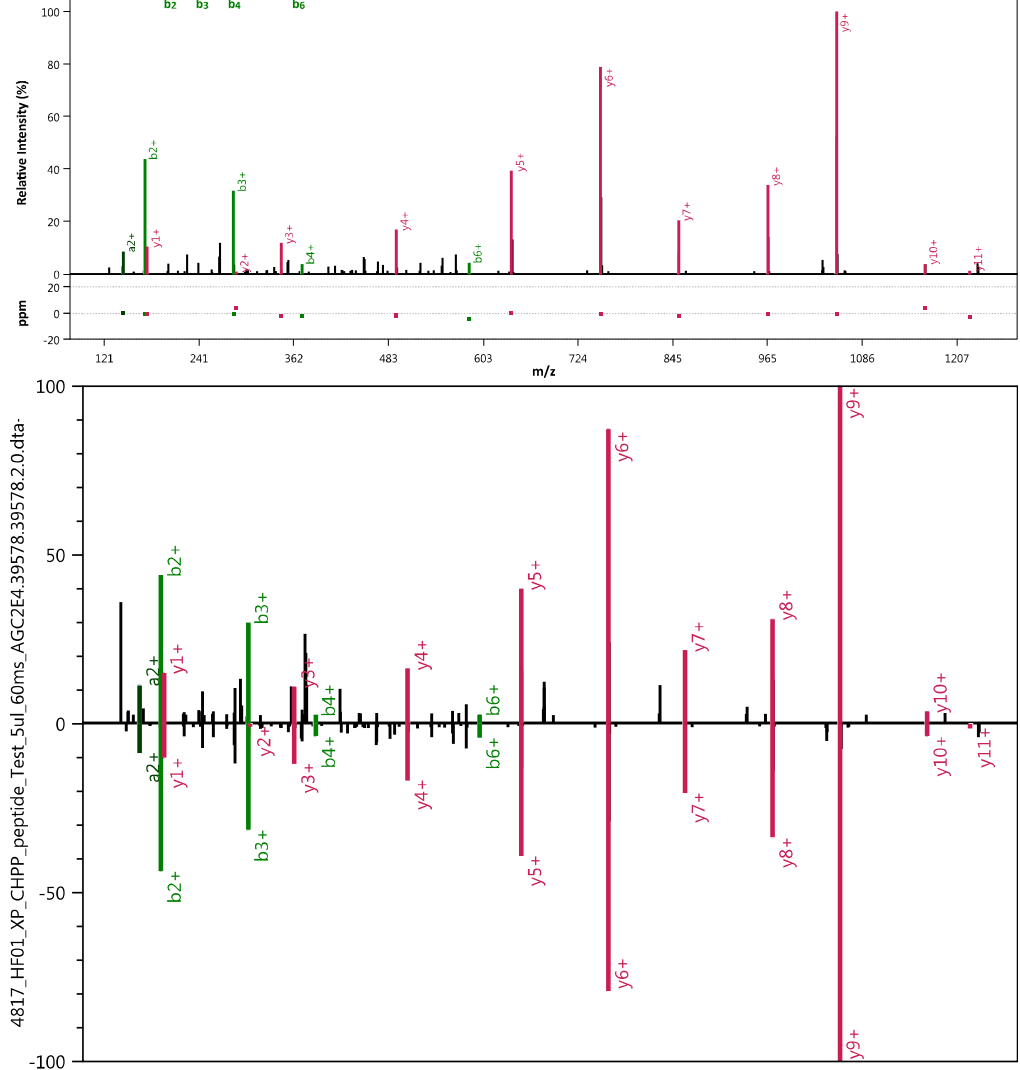


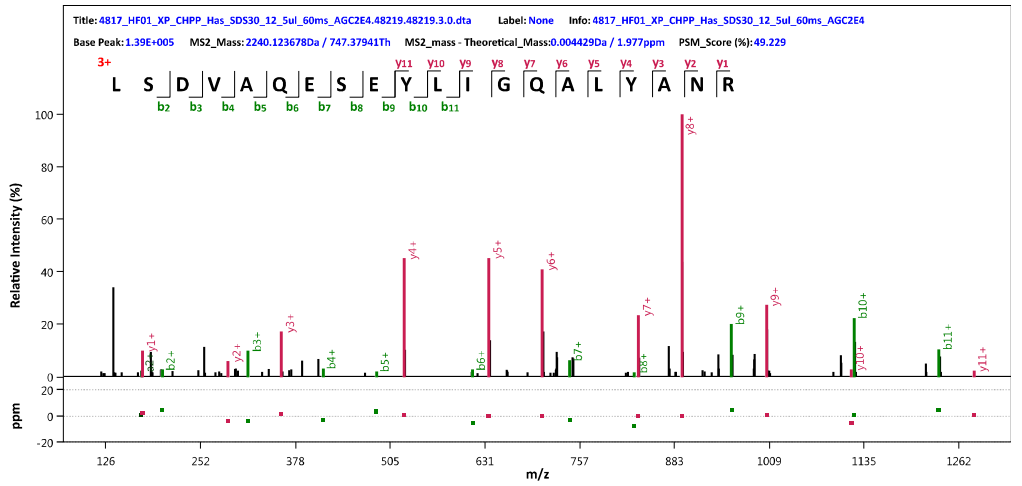

के
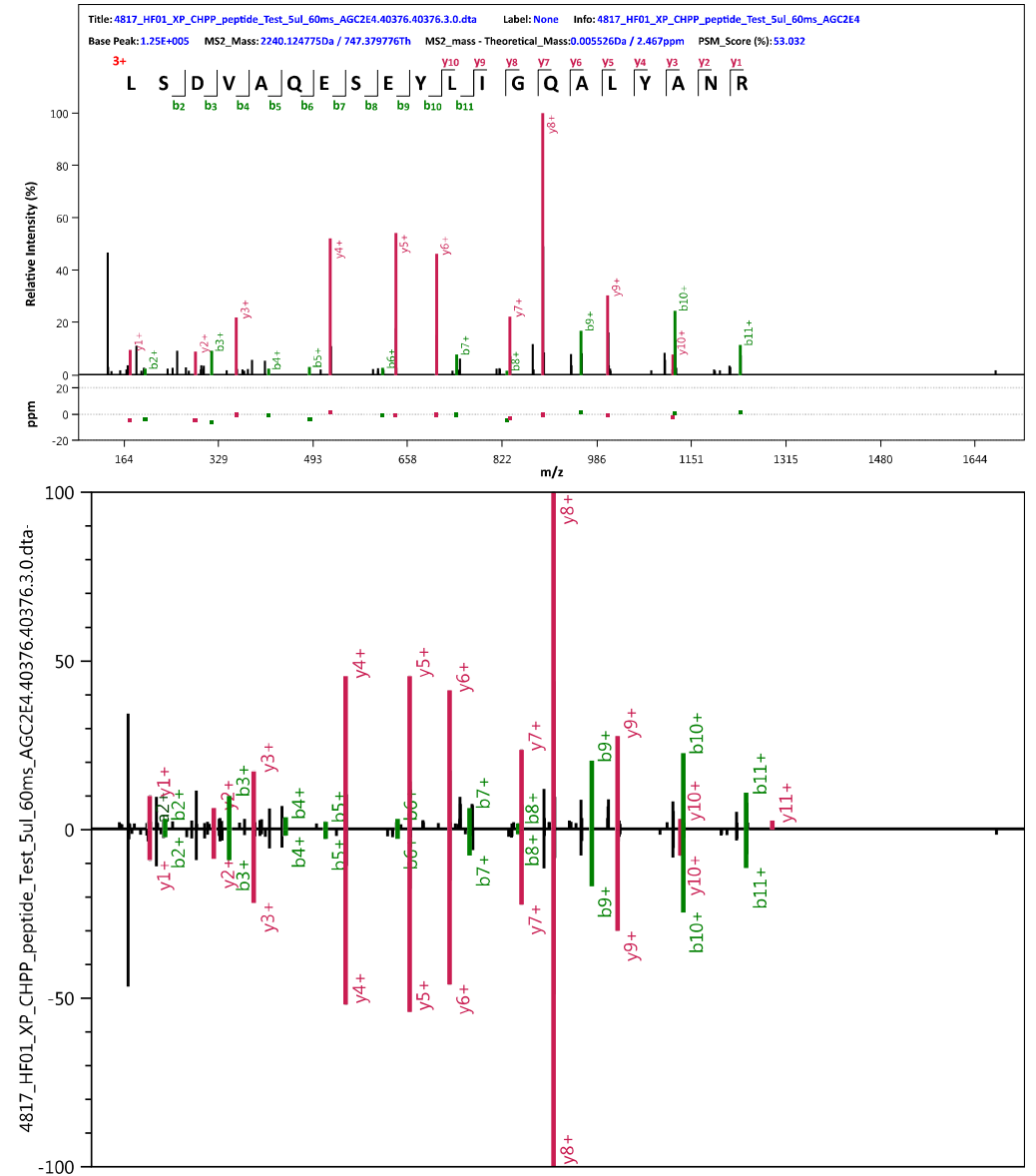

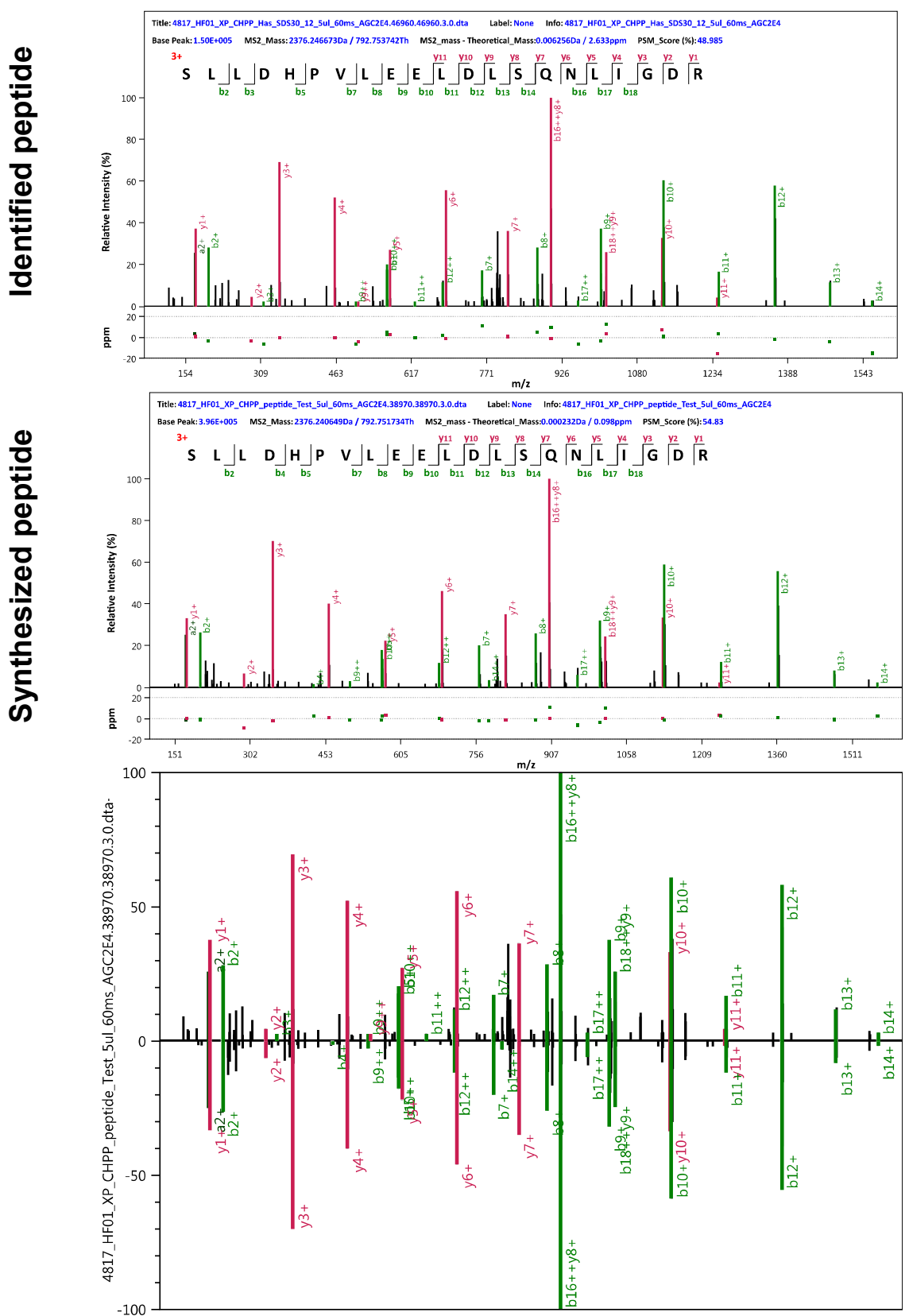
Title: 4817_HF01 XP CHPP Has_SDS10 1_5ul_60ms_AGC2E4.43802.43802.2.0.dta Label: None Info: 4817_HF01_XP_CHPP Has_SDS10_1_5ul_60ms_AGC2E4

lase Peak:8.83E+004 MS2_Mass: 1768.891409Da / 884.949343Th MS2_mass - Theoretical_Mass:0.001025Da/0.579ppm PSM_Score (\%): 31.542

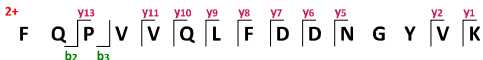

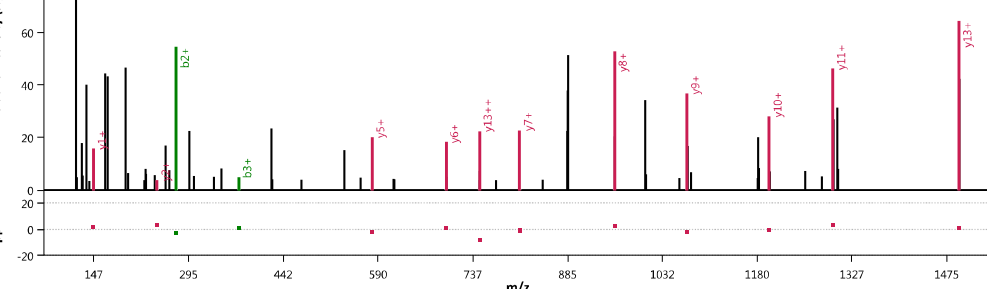

范

The: 4817_HF01_XP_CHPP_peptide_Test_Sul_60ms_AGC2E4.35422.35422.2.0.dta Label: None Info: 4817_HF01_XP_CHPP_peptde_Test_SUl_600ms_AGC2E4

Base Peak:6.85E+007 MS2_Mass: 1768.891014Da / 884.949145Th MS2_mass - Theoretical_Mass:0.000630Da / 0.356ppm PSM_Score (\%):36.646

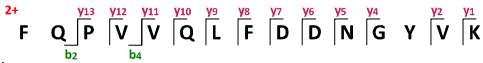
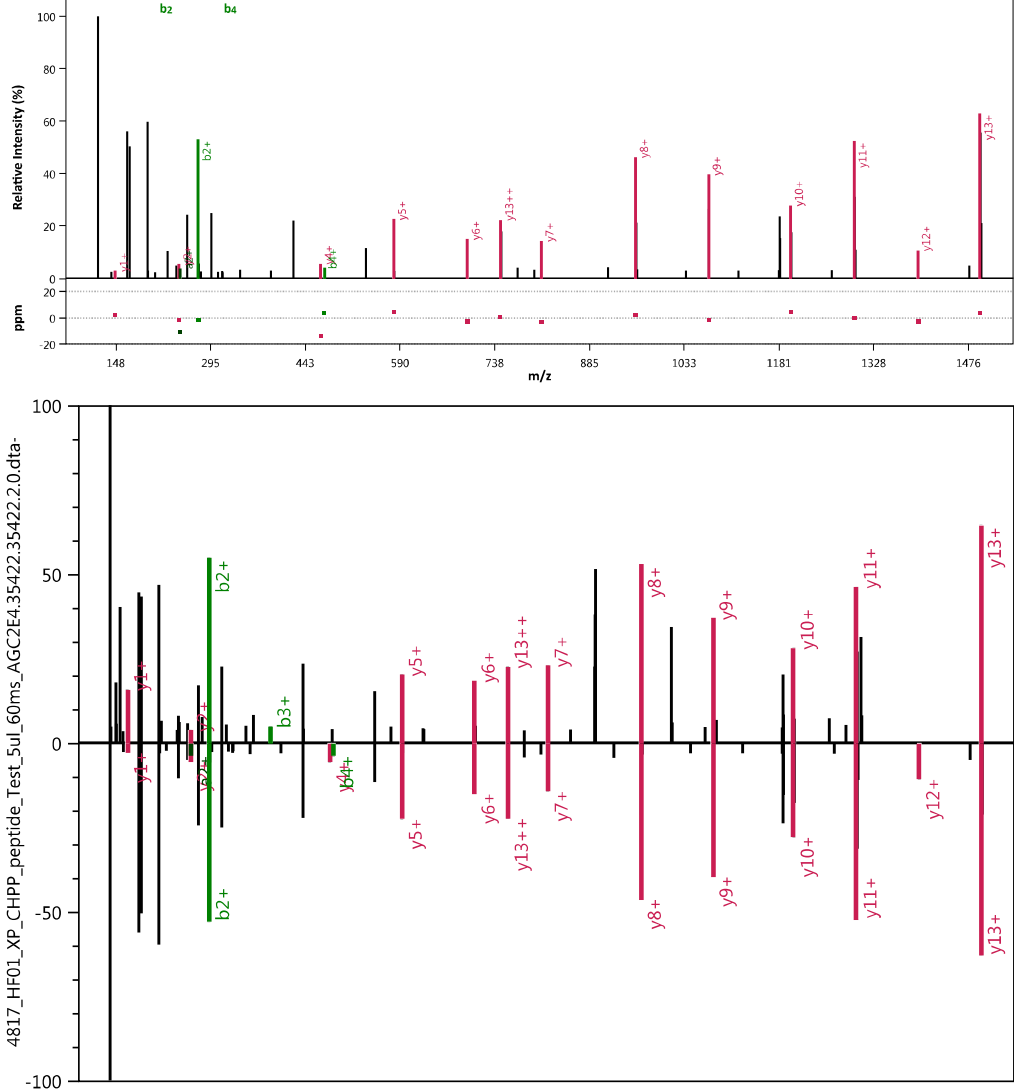


\section{YIWGEYGCPLR}

Title: 4817_HF01_XP_CHPP_Has__DS10_1_5ul_60ms_AGC2E4.37418.37418.2.0.dta Mods: 8,Carbamidomethyl|C](None); Label: None Info:4817_HF01_XP_CHPP_Has__SDS10_1_5ul_60ms_AGC Se Peak:9.28E+004 MS2_Mass: 1413.6656160a / 707.336446Th MS2_mass - Theoretical_Mass:0.003691Da / 2.611ppm PSM_Score (צ): 41.336

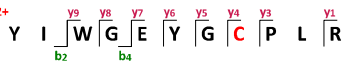

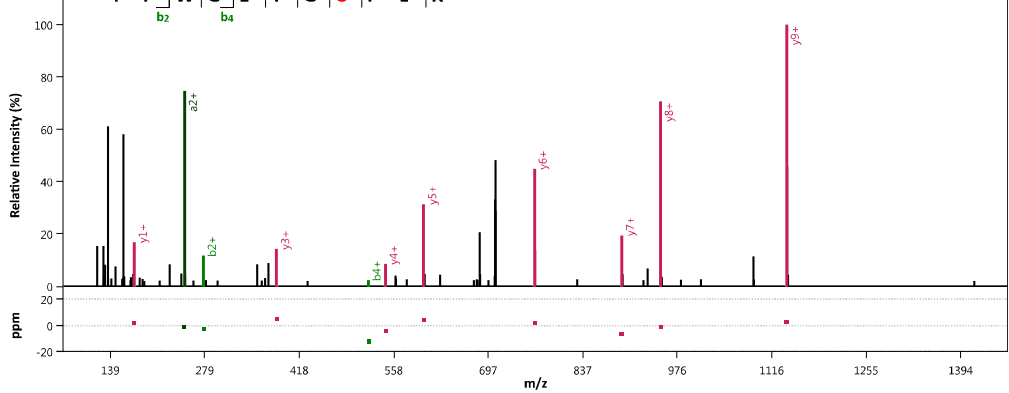

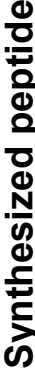

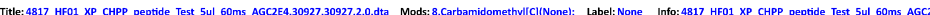
Base Peak: 6.49E+005 MS2_Mass: 1413.66448Da / 707.335878th MS2_mass - Theoretical_Mass:0.002555Da / 1.807ppm PSM_Score (\%): 50.808

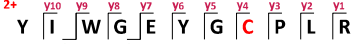
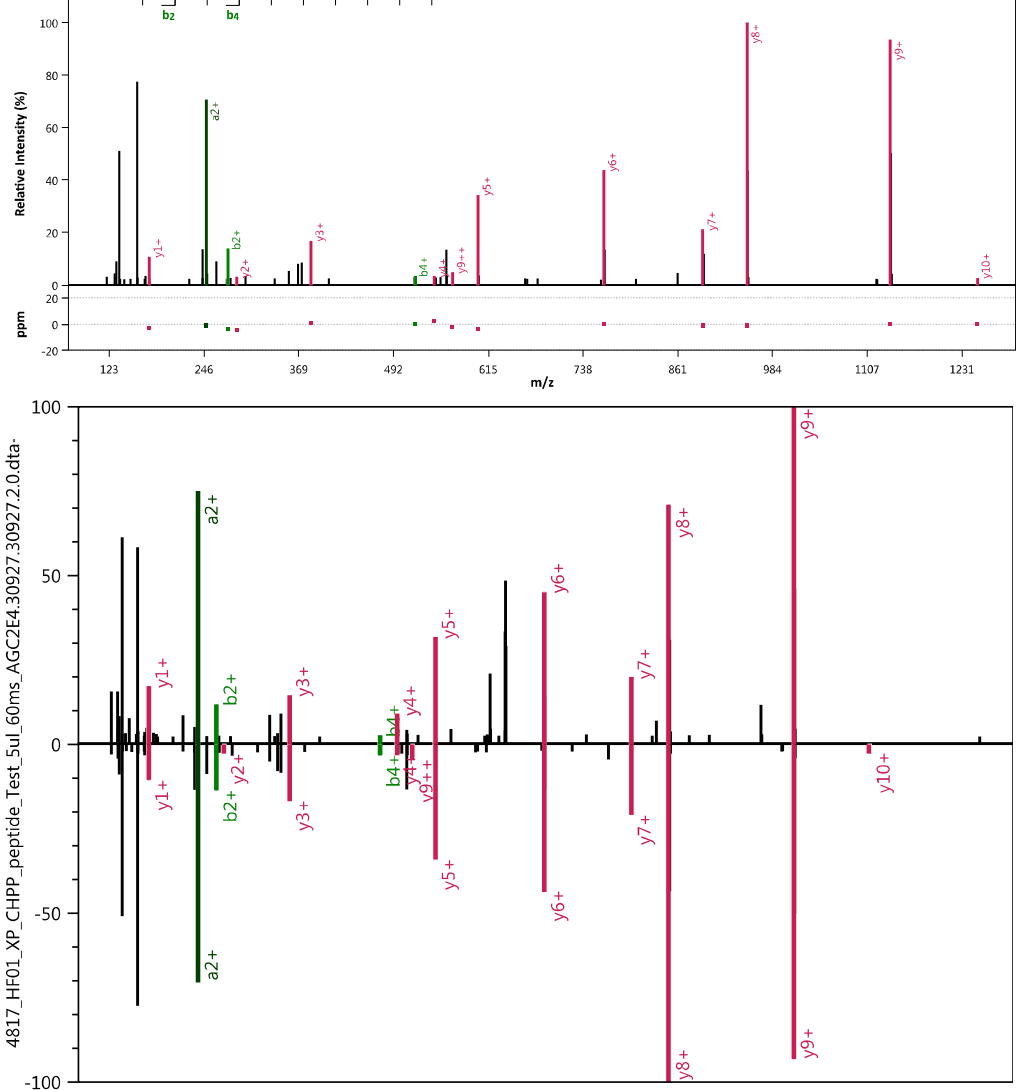
Title: 4817_HF01_XP_CHPP_Has_SDS10_17_5ul_60ms_AGC2E4.43809.43809.2.0.dta Label: None Info: 4817_HF01_XP_CHPP_Has_SDS10_17_Sul_60ms_AGC2E4

Peak: 5.77E+004 MS2_Mass: 1109.730943Da / 555.36911Th MS2_mass - Theoretical_Mass:0.001742Da / 1.570ppm PSM_Score (\%):39.923

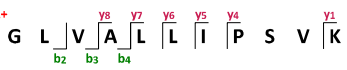

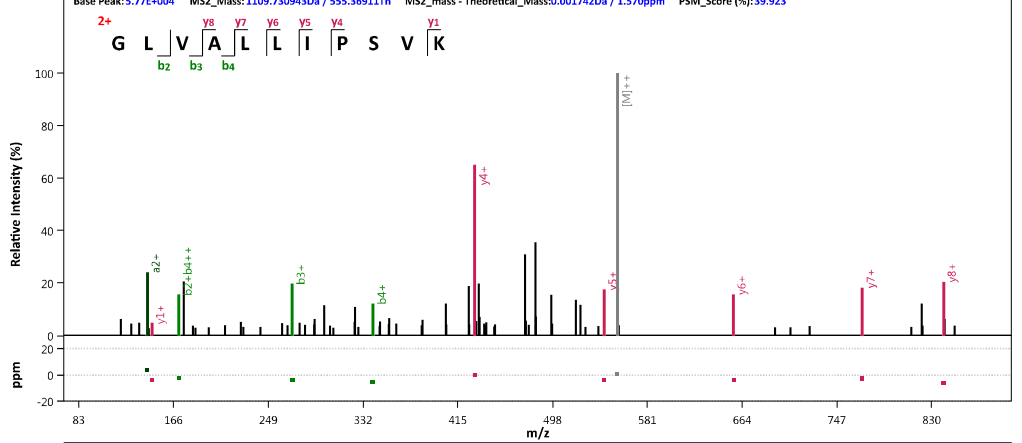

Title: 4817_HF01_XP_CHPP_Peptide_Test__5ul_60ms_AGC2E4.36879.36879.2.0.dta Label: None Info: 4817_HF01_XP_CHPP_peptide_Test_5ul_60ms_AGC2E4 Base Peak:2.29E+008 MS2_Mass: 1109.730576Da / 555.368926Th MS2_mass - Theoretical_Mass:0.001374Da/1.238ppm PSM_Score (\%):67.764

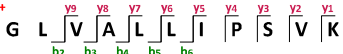
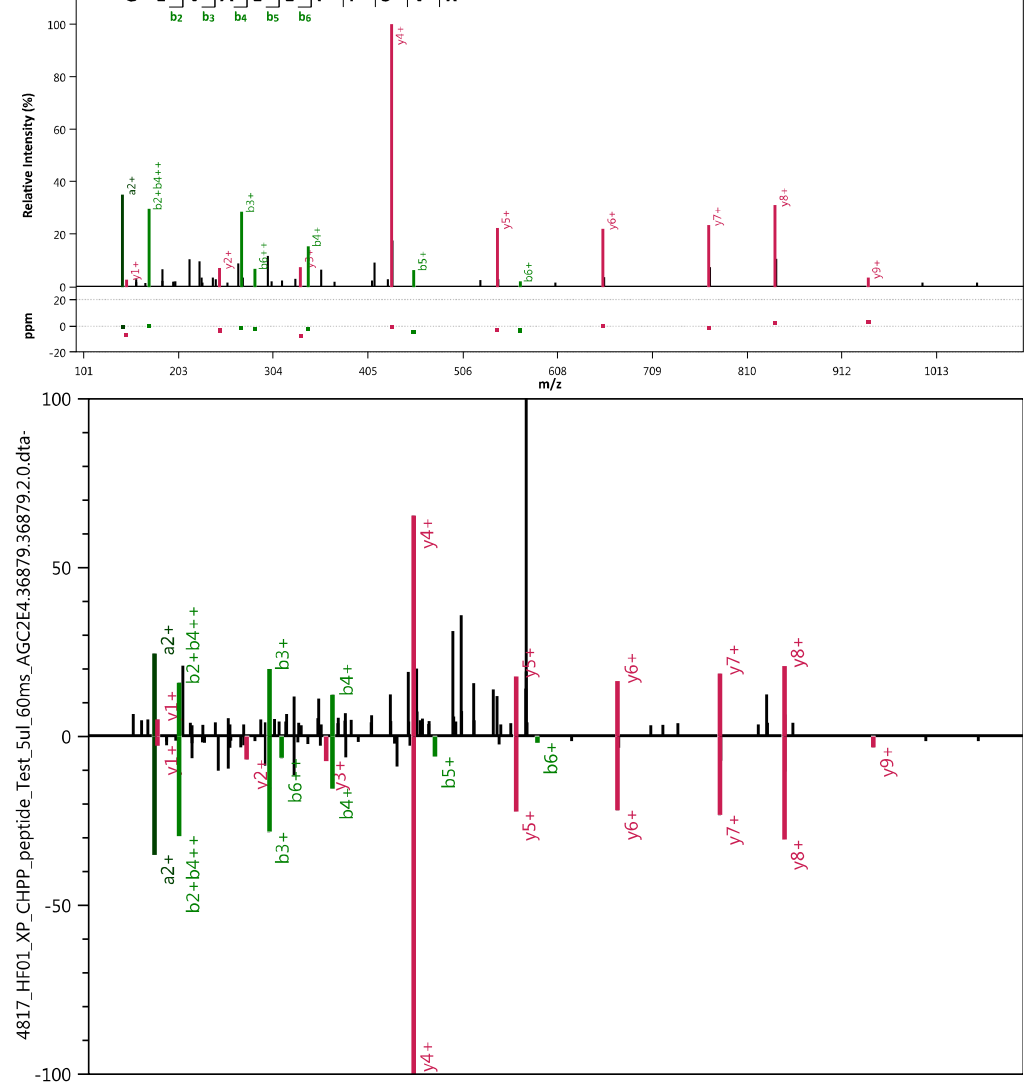

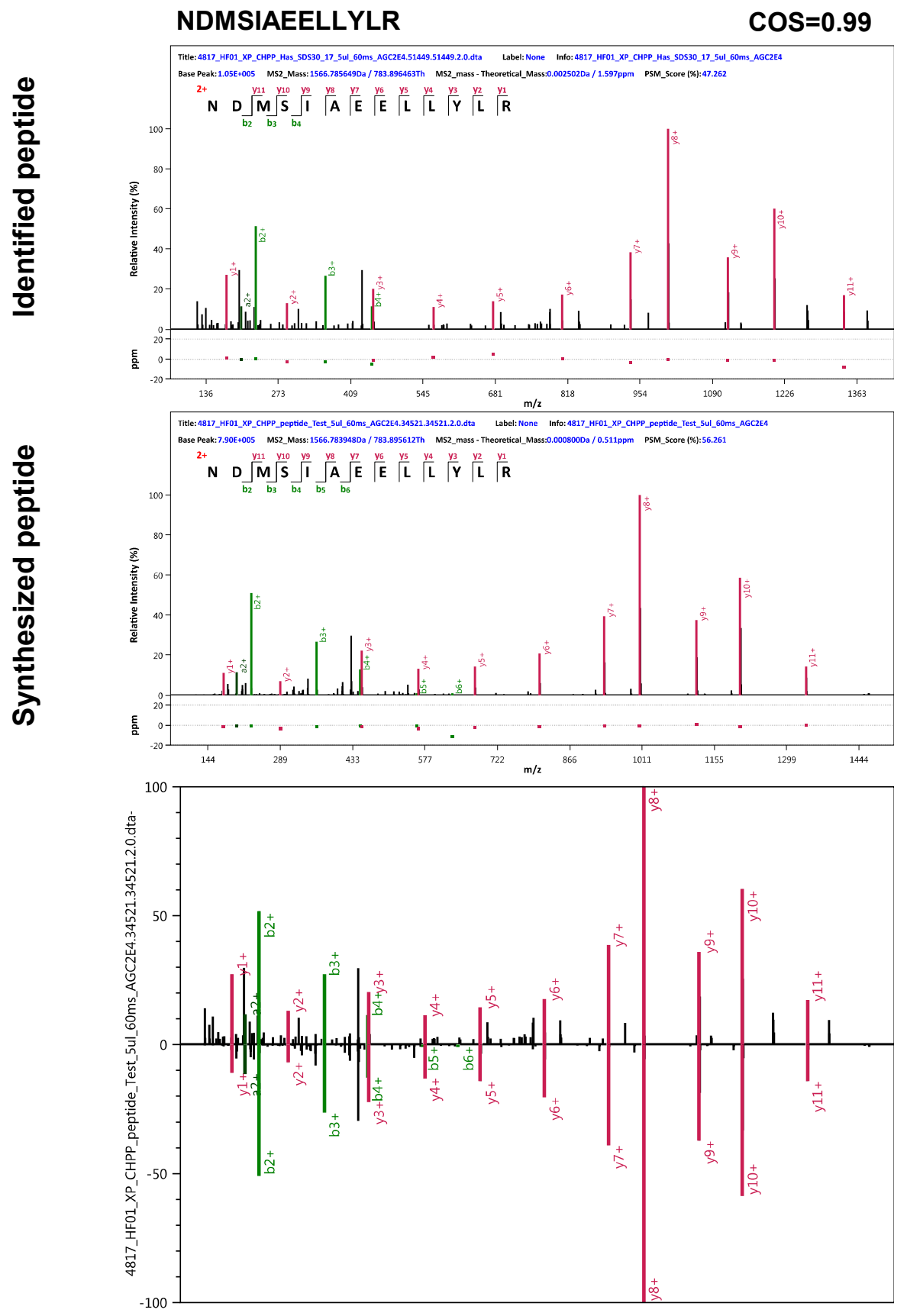

S-37 


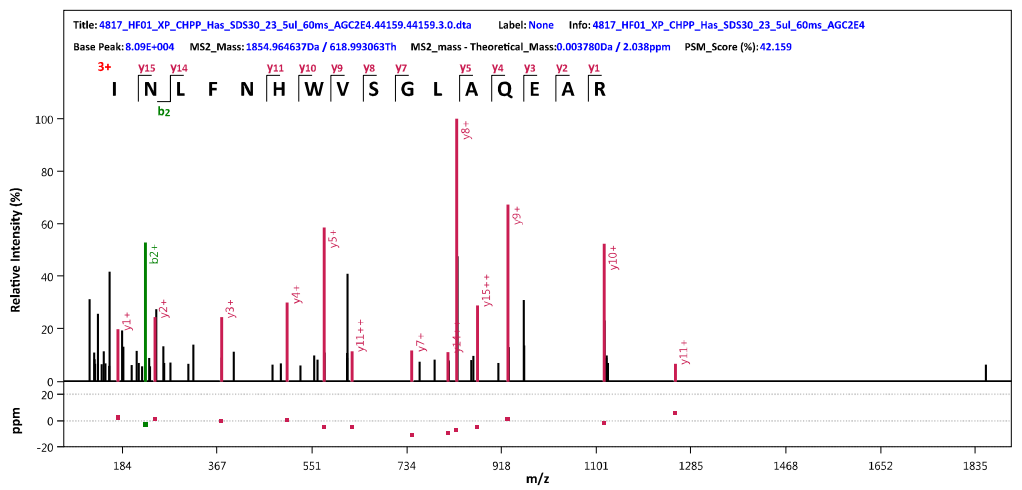

कृ
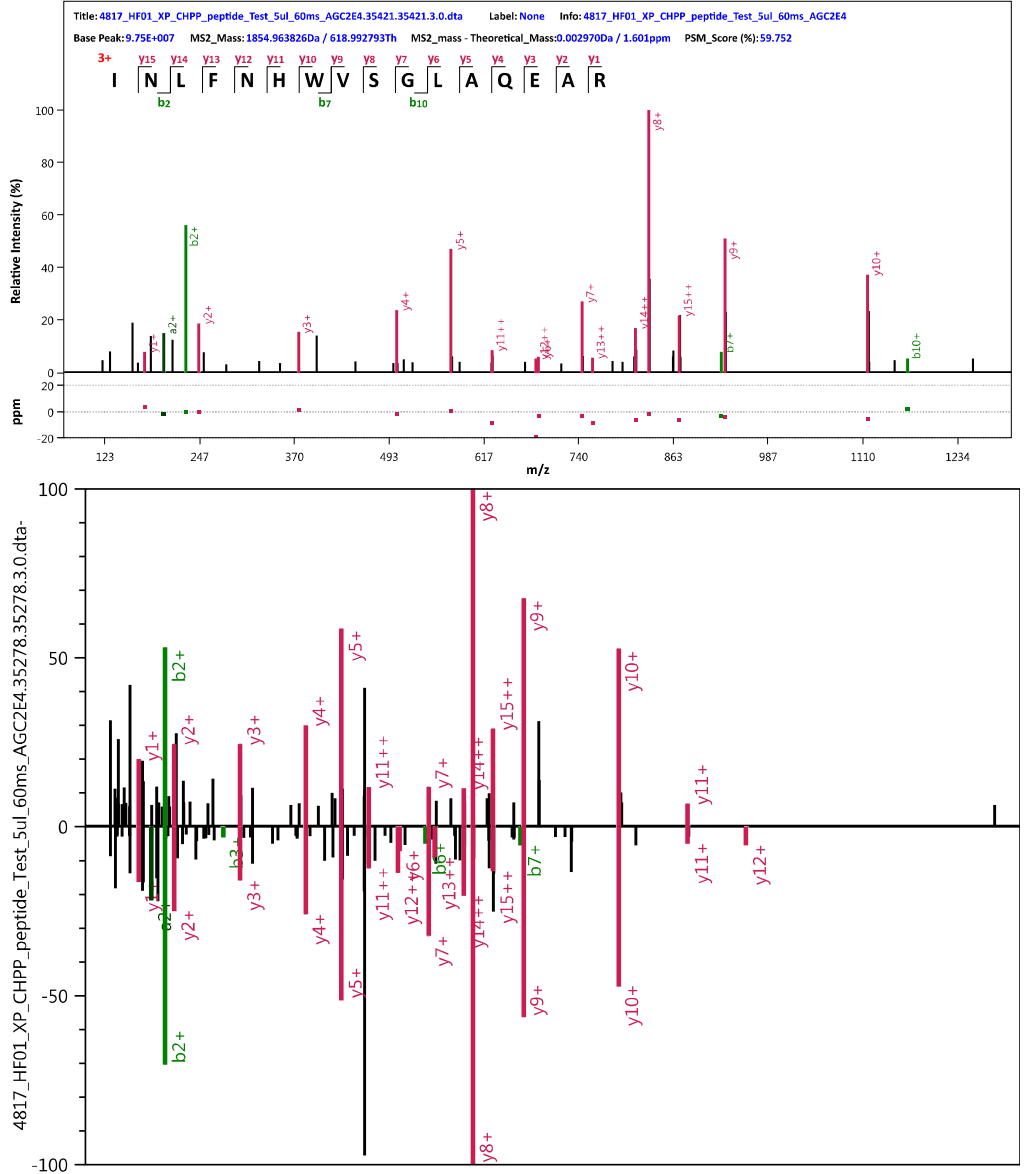


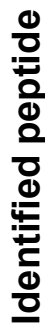

Title: 4817_HF01_XP_CHPP_Has_SOS30_23_5ul_60ms_AGC2E4.29823.29823.2.0.dta Label: None Info: 4817_HF01_XP_CHPP_Has__SDS30_23_5ul_60ms_AGC2E4 Base Peak: 1.14E+005 MS2_Mass: 1041.64292Da / 521.325098Th MS2_mass - Theoretical_Mass:0.001468Da / 1.409ppm PSM_Score (\%):39.409

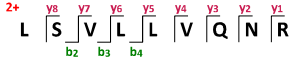

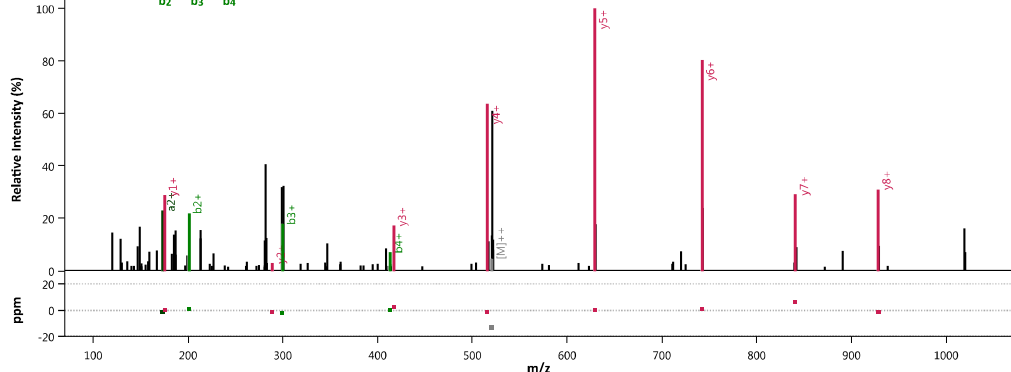

e: 4817 HF01_XP_CHPP_peptide_Test_5ul_60ms_AGC2E4.22342.22342.2.0.dta Label: None Info: 4817_HF01_XP_CHPP_peptide_Test_5ul_60ms_AGC2E4 Base Peak:4.91E+005 MS2_Mass: 1041.642584Da / 521.32493Th MS2_mass - Theoretical_Mass:0.001131Da / 1.086ppm PSM_Score (צ): 53.866

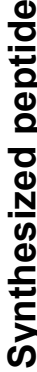

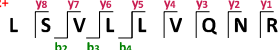
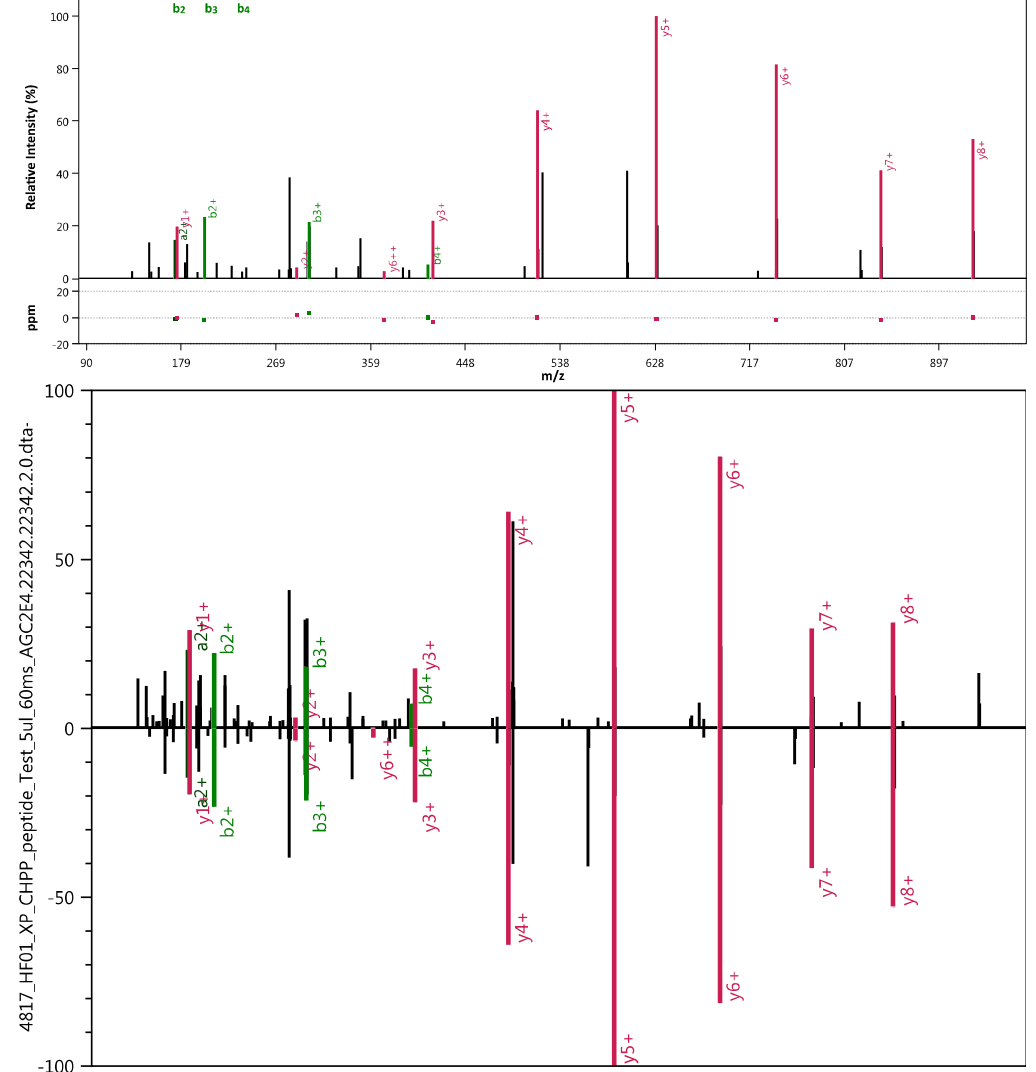

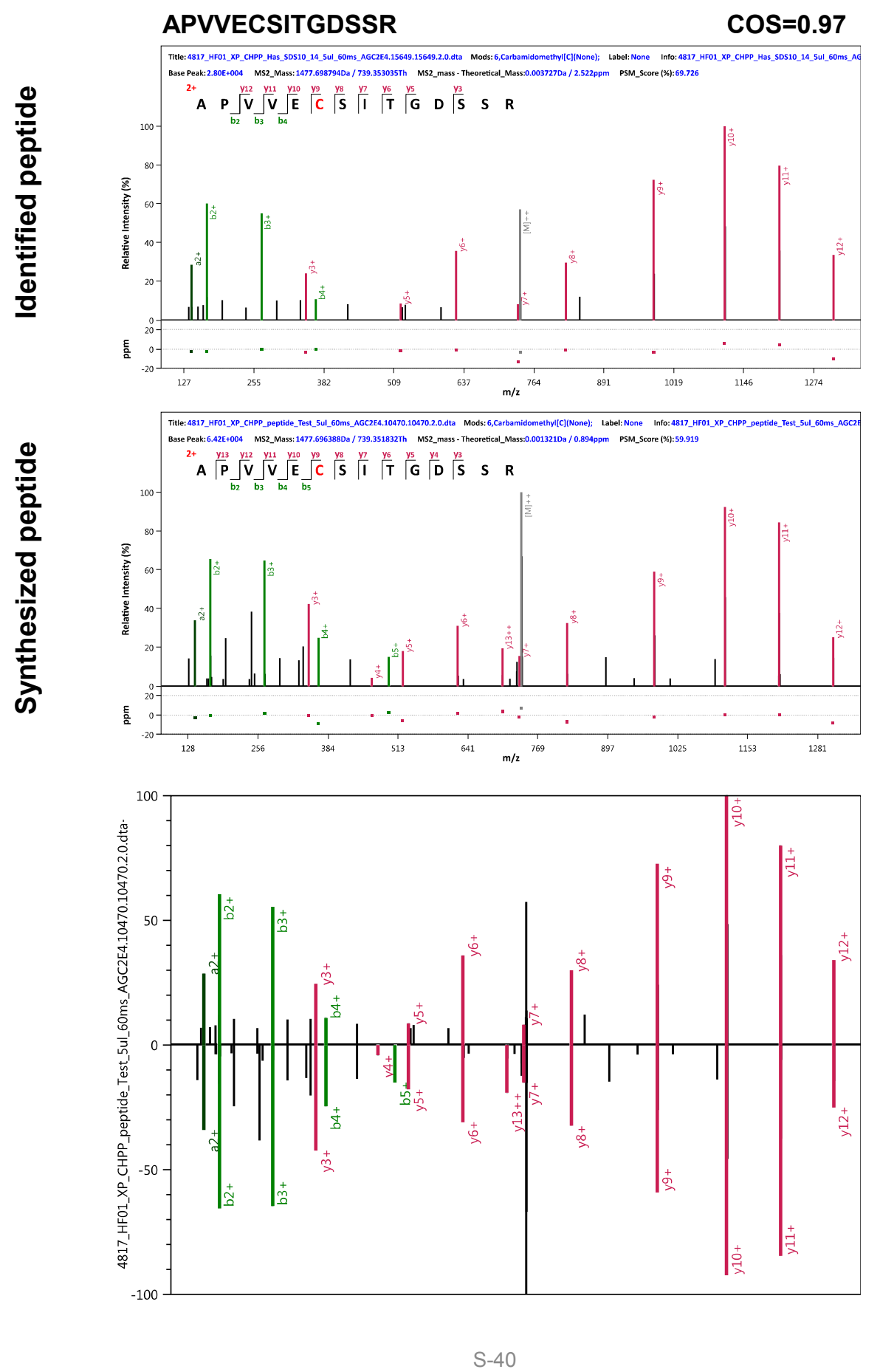
GLPADTSSSSSSSER

$\cos =0.99$

Title: 4817_HF01_XP_CHPP_Has_Tricine10_7_5ul_60ms_AGC2E4.5564.5564.2.0.dta Label: None Info: 4817_HF01_XP_CHPP_Has_Tricine10_7_5ul_60ms_AGC2E4

Base Peak: 7.27E+004 MS2_Mass: 1467.658447Da/ 734.332862Th MS2_mass - Theorettcal_Mass:0.002744Da / 1.870ppm PSM_Score (\%): 40.817

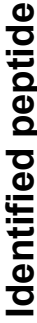

GLL L

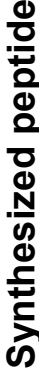

Title: 4817_HF01_XP_CHPP_peptide_Test_5ul_60ms_AGC2E4.4365.4365.2.0.dta Label: None Info: 4817_HF01_XP_CHPP_peptide_Test_5ul_60ms_AGC2E4

Base Peak: 1.20E+005 MS2_Mass: 1467.656492Da / 734.331884Th MS2_mass - Theoretical_Mass:0.000789Da / 0.537ppm PSM_score (\%): 54.149

${ }^{2+}$ G L L
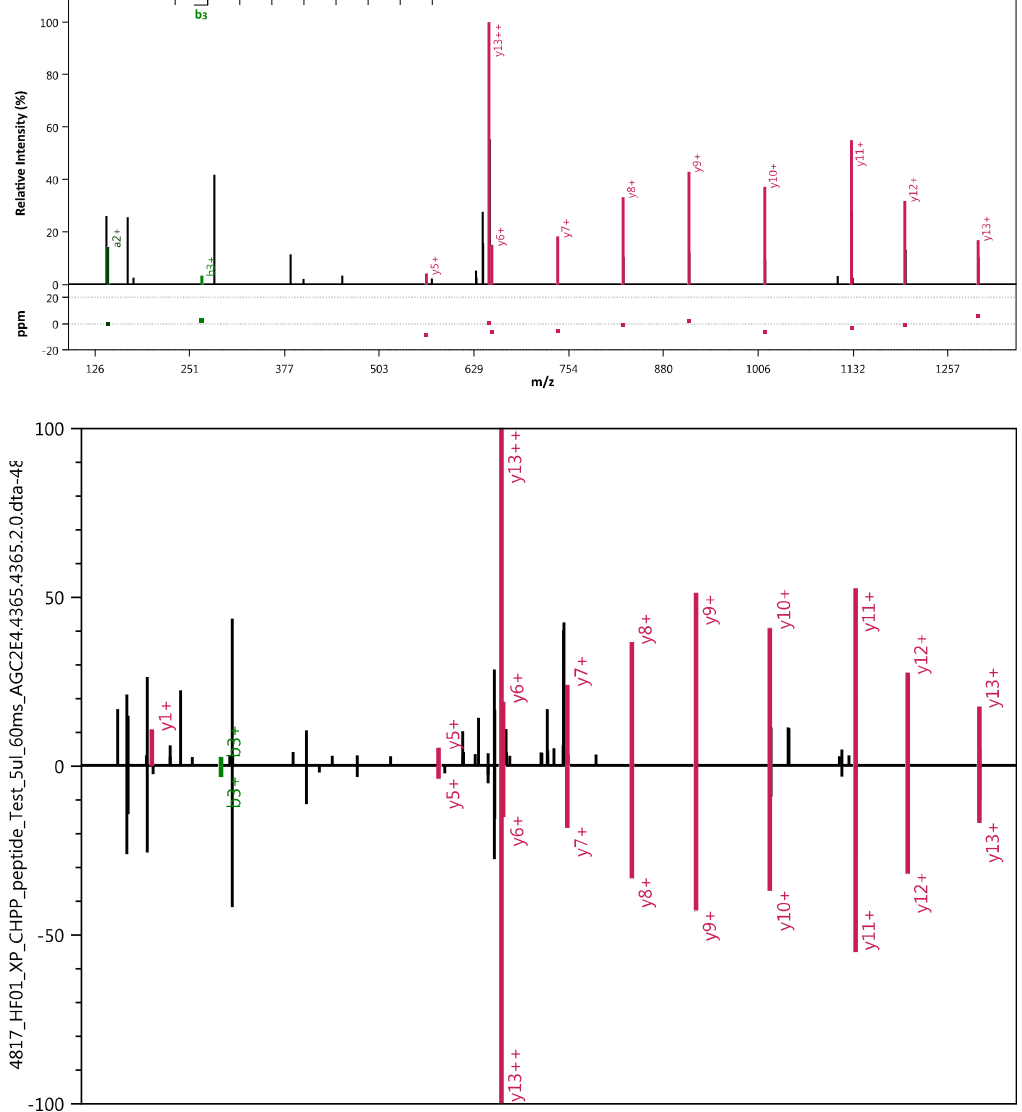


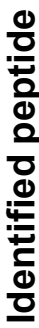

Title: 4817 HF01_XP_CHPP_Has_SDS10 13_5ul 60ms_AGC2E4.34599.34599.2.0.dta Label: None Info:4817_HF01 XP_CHPP_Has_SDS10_13_5ul_60ms_AGC2E4

Base Peak:1.67E+005 MS2_Mass: 1242.6505780a / 621.828927Th MS2_mass - Theoretical_Mass:0.002923Da/2.352ppm PSM_Score (\%):37.683

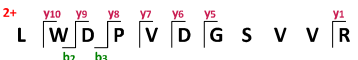

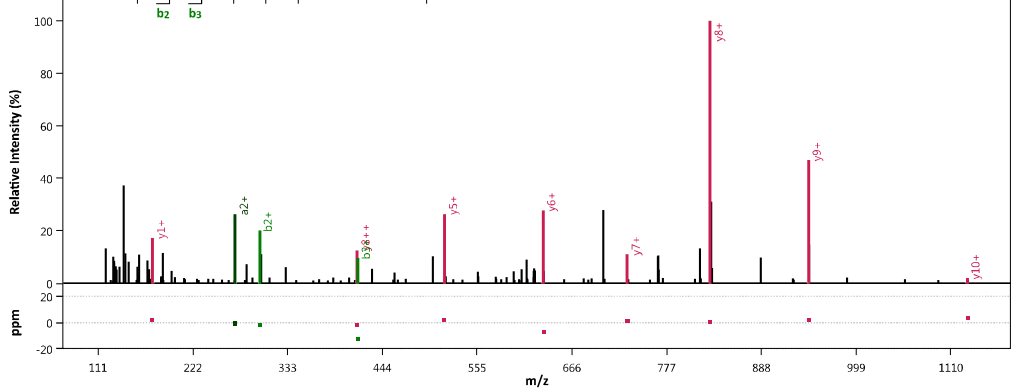

$\frac{0}{0}$
$\frac{1}{0}$
$\frac{0}{0}$
$\frac{0}{0}$
$\frac{N}{0}$
के
के

Title: 4817_HF01_XP_CHPP_peptide_Test_5ul_60ms_AGC2E4.20353.20353.2.0.dta Label: None Info: 4817_HF01_XP_CHPP_peptide_Test_5ul_60ms_AGC2E4 Base Peak: 9.49E+005 MS2_Mass: 1242.647968Da / 621.827622Th MS2_mass - Theoretical_Mass:0.000312Da / 0.251 ppm PSM_Score (\%): 56.598

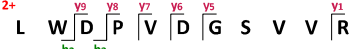
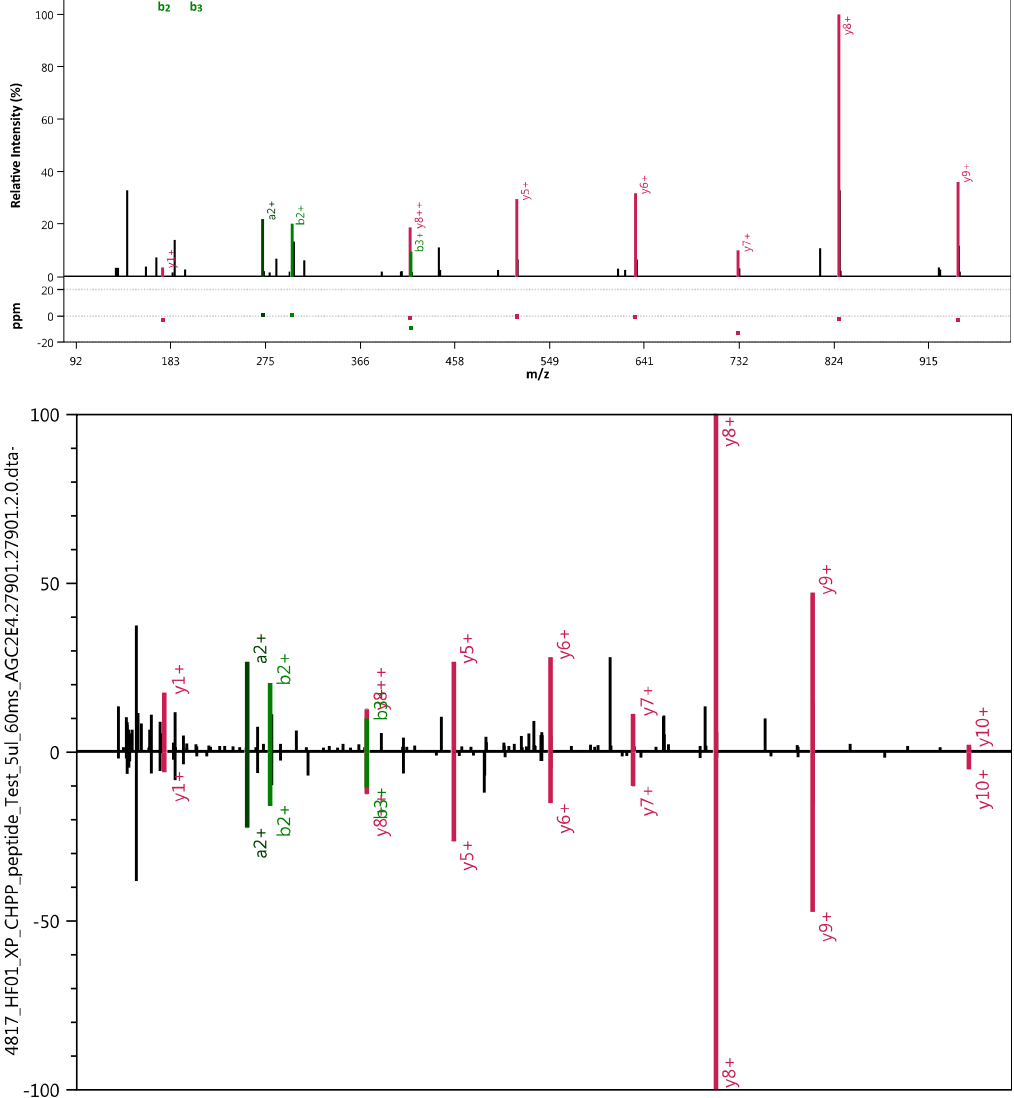
Title: 4817_HF01_XP_CHPP_Has_SDS30_21_5ul_60ms_AGC2E4.12911.12911.2.0.dta Label: None Info: 4817_HF01_XP_CHPP_Has_SDS30_21_5ul_60ms_AGC2E4

Base Peak:3.21E+004 MS2_Mass: 1326.592724Da / 663.8Th MS2_mass - Theoretical_Mass:0.003236Da / 2.439ppm PSM_Score (\%): 45.815

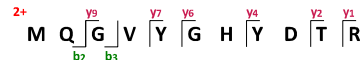

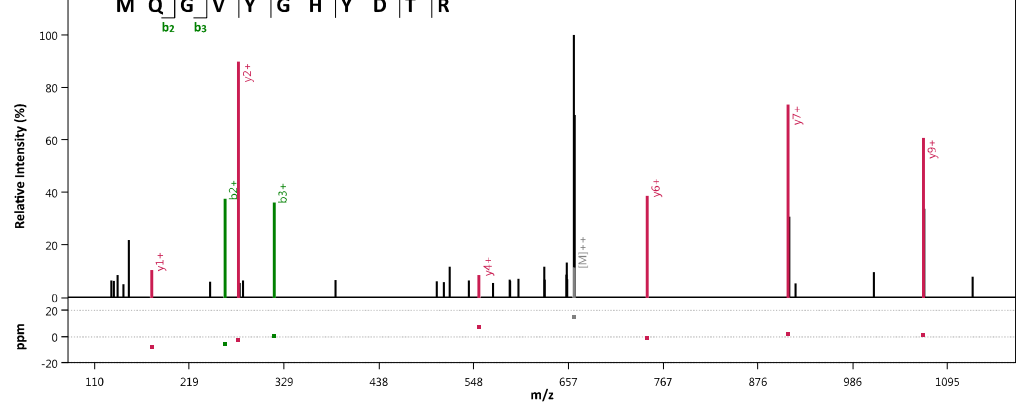

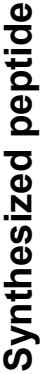
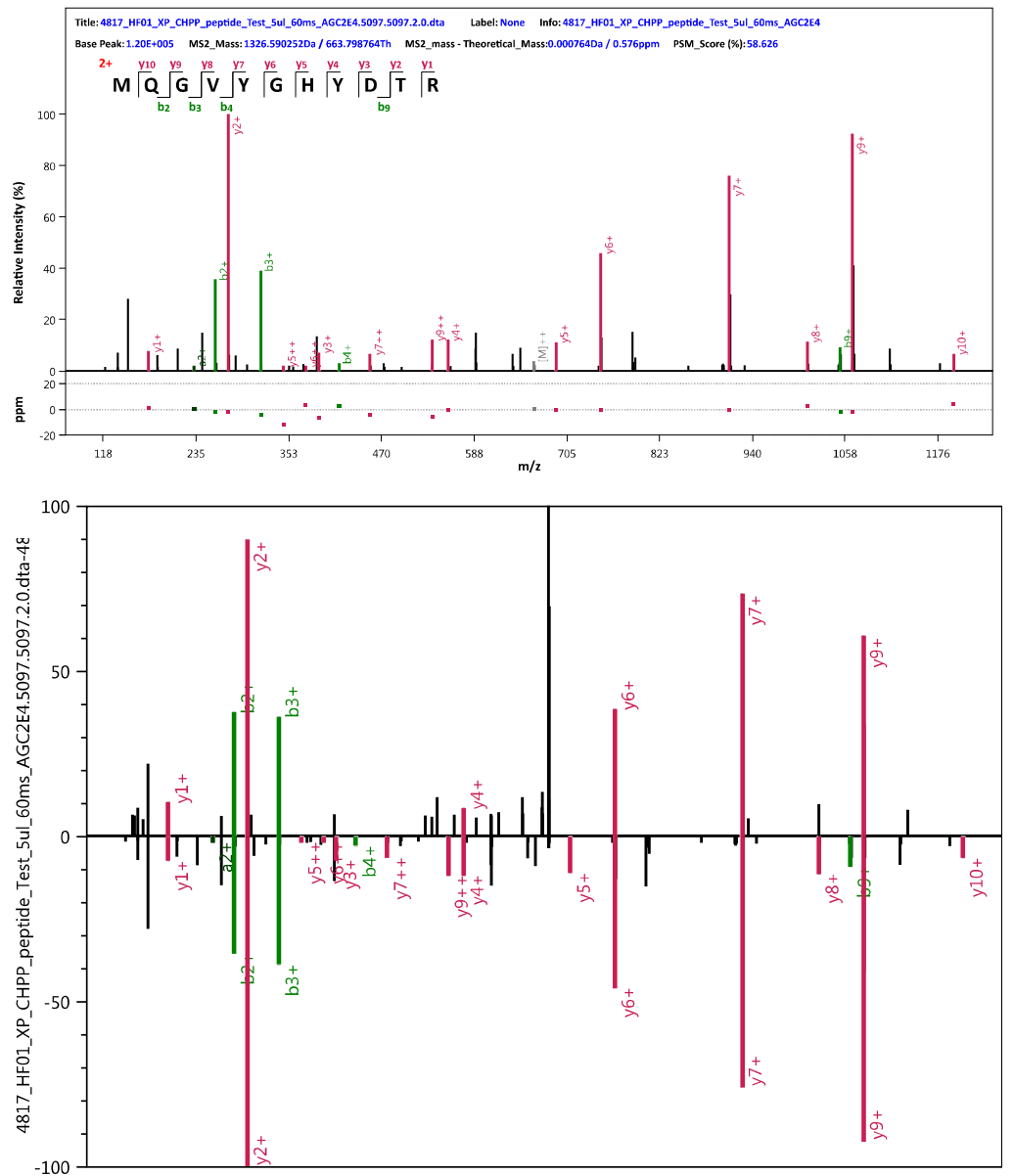


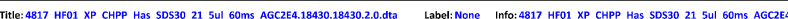
Base Peak: 6.93E+004 MS2_Mass: 1577.732724Da / 789.37Th MS2_mass - Theoretical_Mass:0.010387Da / 6.583ppm PSM_Score (\$): 34.415

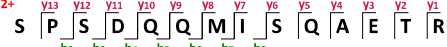

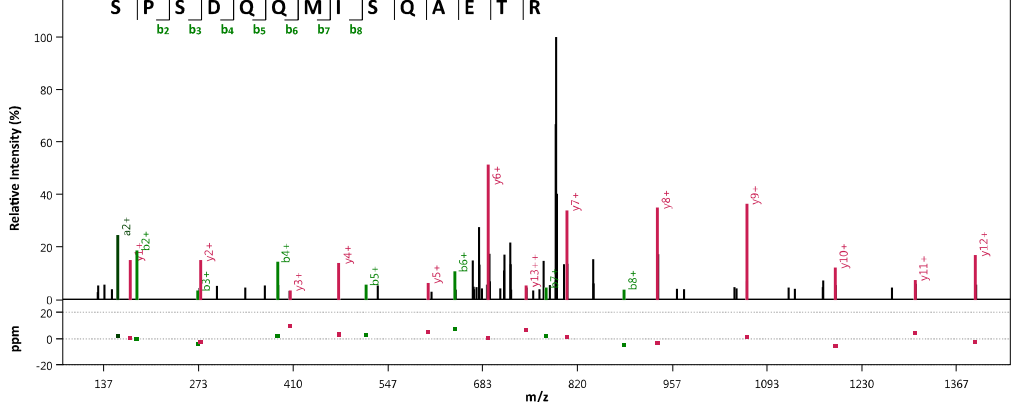

을
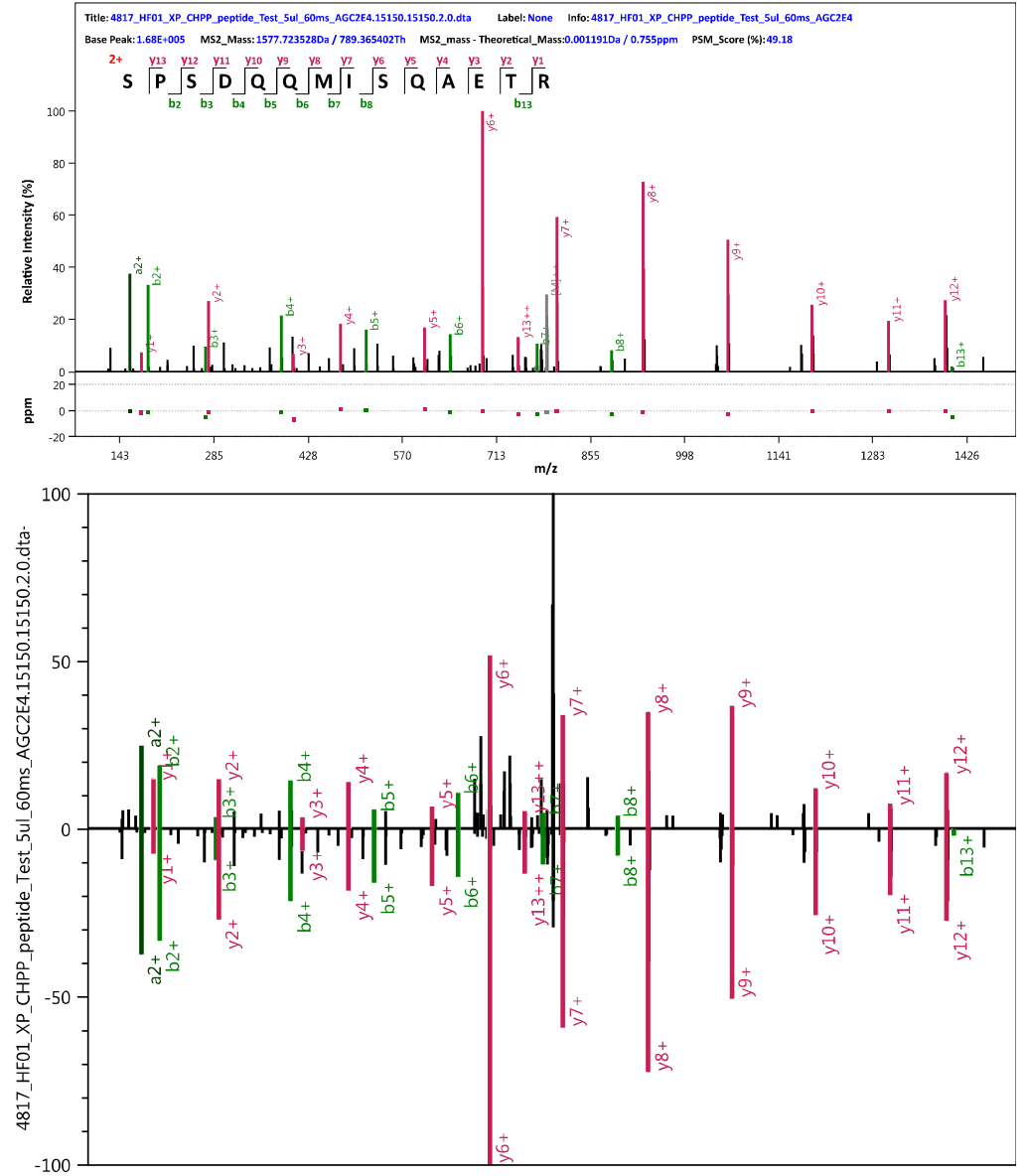
Title: 4817_HF01_XP_CHPP_Has_Tricine10_4_6ul_60ms_AGC2E4.14678.14678.2.0.dta Label: None Info: 4817_HF01_XP_CHPP_Has_Tricine10_4_6ul_60ms_AGC2E4

ase Peak:5.27E+004 MS2 Mass: 1057.602363Da / 529.30482Th MS2_mass - Theoretical_Mass:0.002380Da / 2.251ppm PSM Score (\%): 26.41

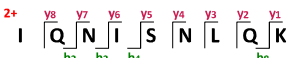

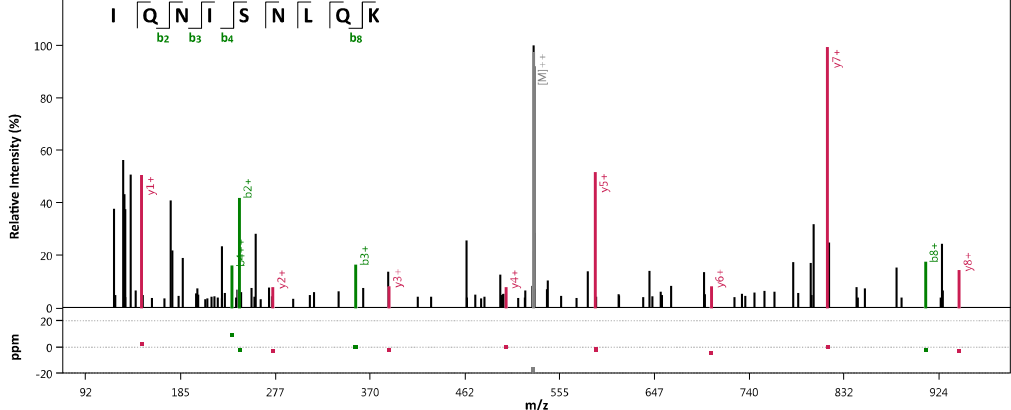

$\frac{1}{0}$
$\frac{0}{0}$
0
0
$\frac{0}{0}$
$\frac{1}{N}$
$\frac{1}{9}$
$\frac{1}{5}$
$\frac{5}{2}$

Title: 4817 HF01 XP CHPP peptide Test 5ul 60ms AGC2E4.11171.11171.2.0.dta Label: None Info: 4817 HF01 XP CHPP peptide Test 5ul 60ms AGC2E4 Base Peak: 2.67E +007 MS2_Mass: 1057.600724Da / 529.304Th MS2_mass - Theoretical_Mass:0.000741Da/0.701ppm PSM_Score (\%):46.623

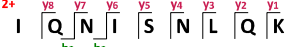
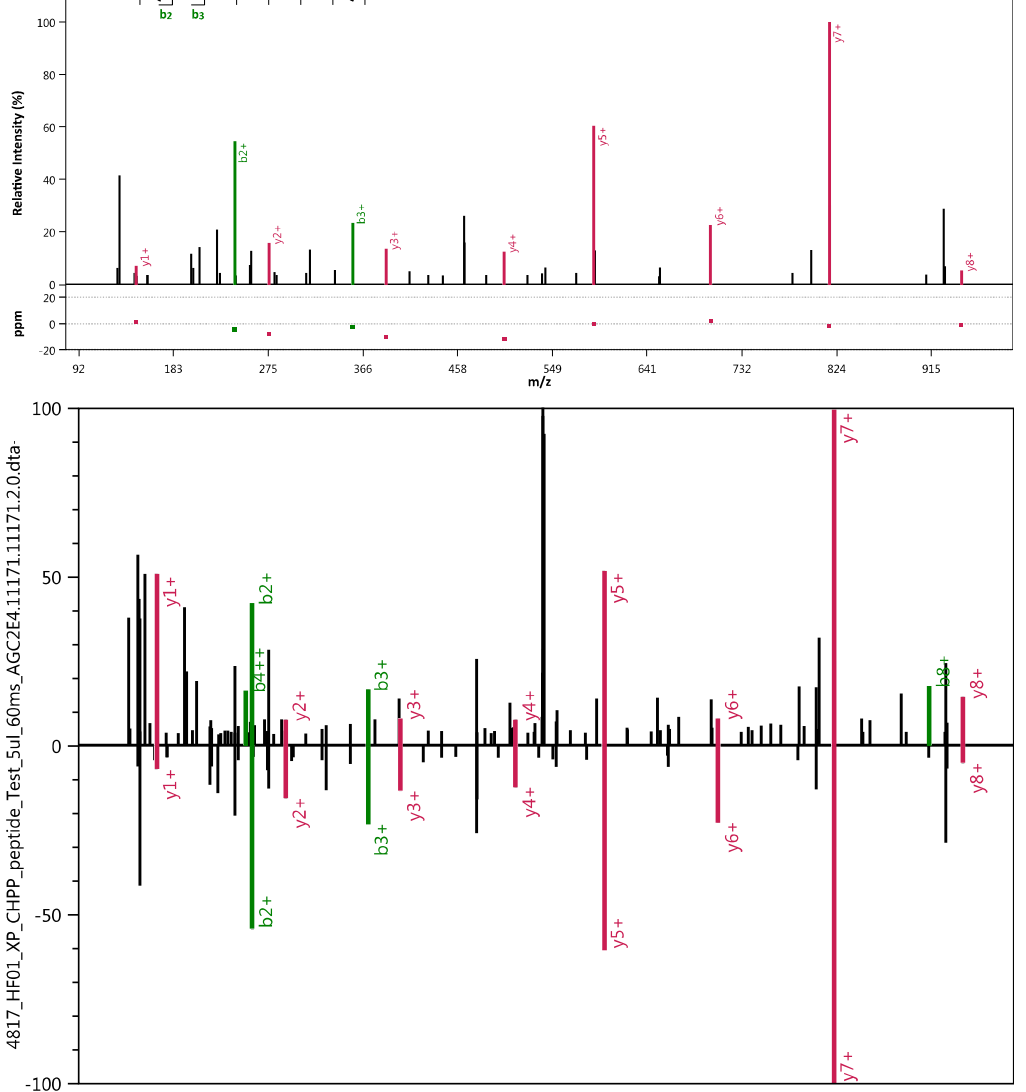

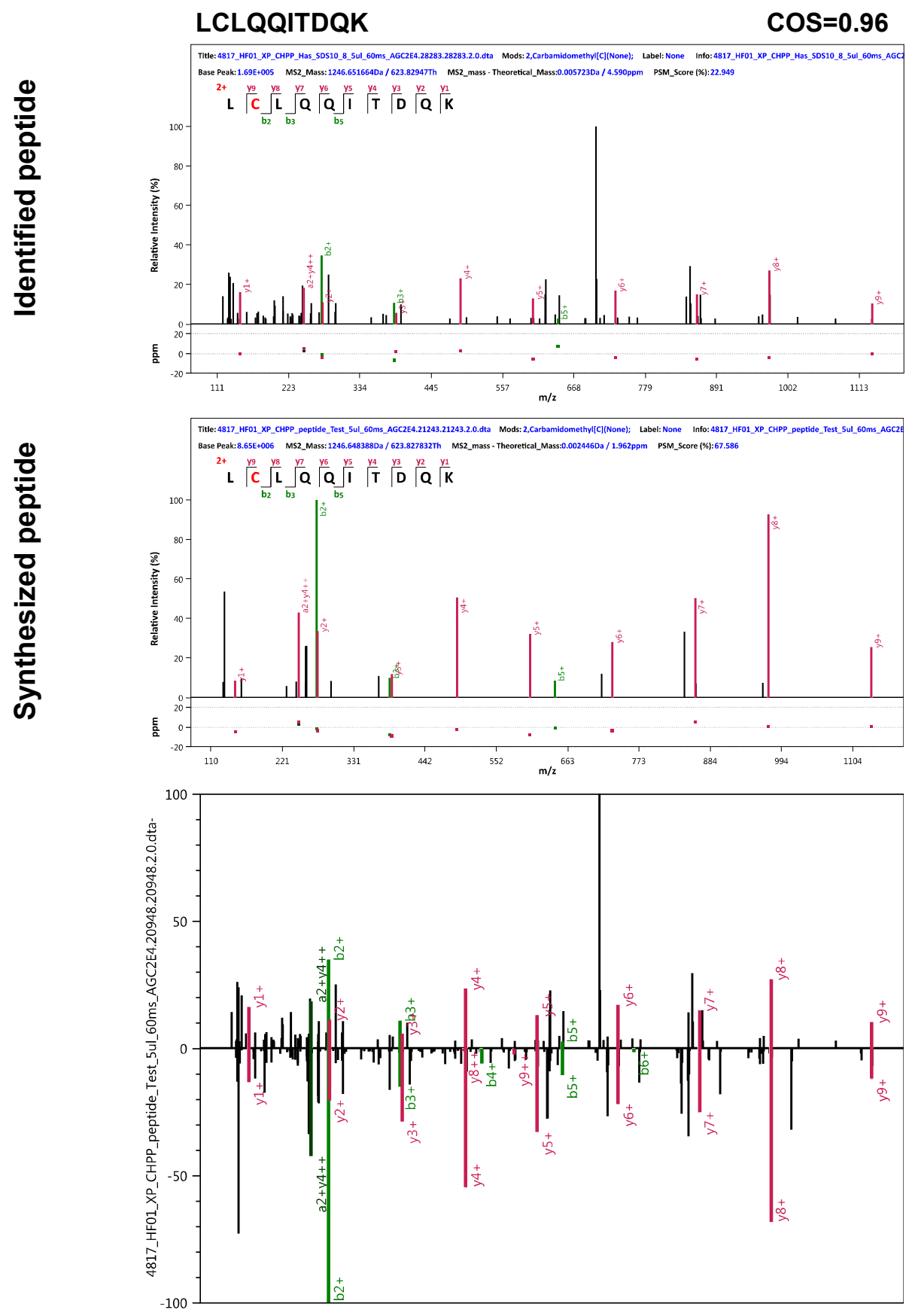

S-46 


\section{LISILGDAR}

Title: 4817_HF01_XP_CHPP_Has_SOS10_8_5Ul_60ms_AGC2E4.34264.34264.2.0.dta Label:None Info: 4817_HF01_XP_CHPP_Has_SDS10_8_5Ul_60ms_AGC2

Base Peak:2.61E+005 MS2_Mass:957.575029Da / 479.291153Th MS2_mass - Theoretical_Mass:0.002320Da/2.423ppm PSM__core (\%):54.661

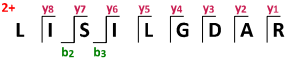

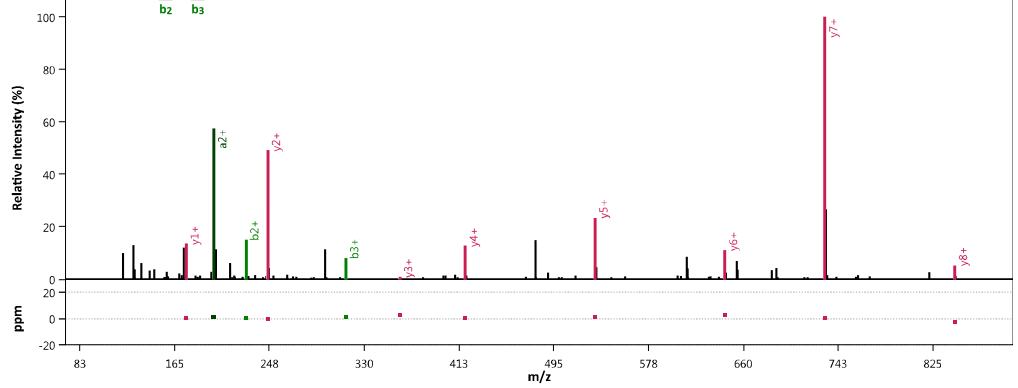

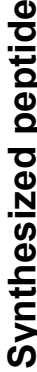

Title: 4817_HF01_XP_CHPP_peptide_Test_5ul_60ms_AGC2E4.23888.23888.2.0.dta Label: None Info: 4817_HF01_XP_CHPP_peptide_Test_5ul_60ms_AGC2E4

Base Peak: 3.45E+005 MS2_Mass: 957.573796Da / 479.290536Th MS2_mass - Theoretical_Mass:0.001086Da / 1.134ppm PSM_Score (\%):55.025

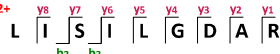
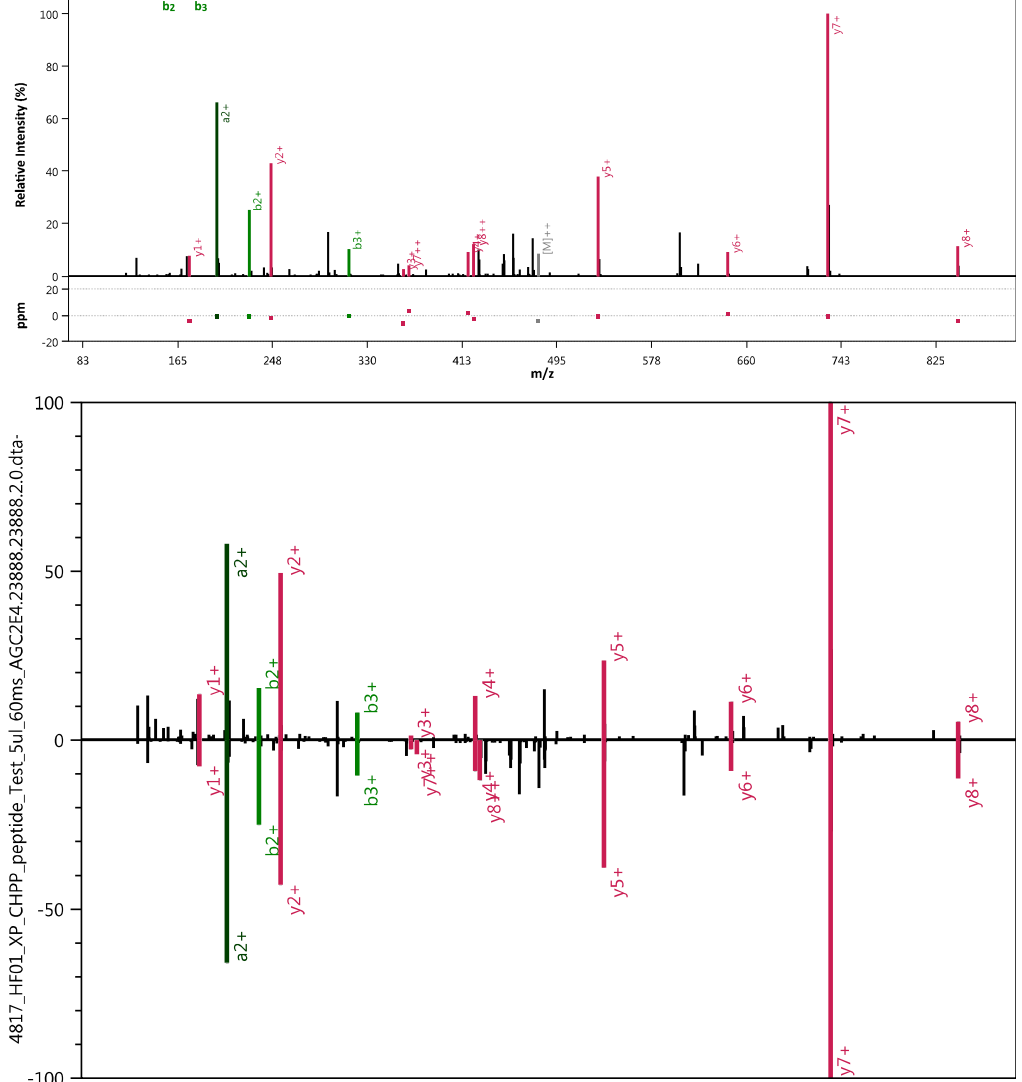
FMTLLAMSLQNLK

$\cos =0.96$
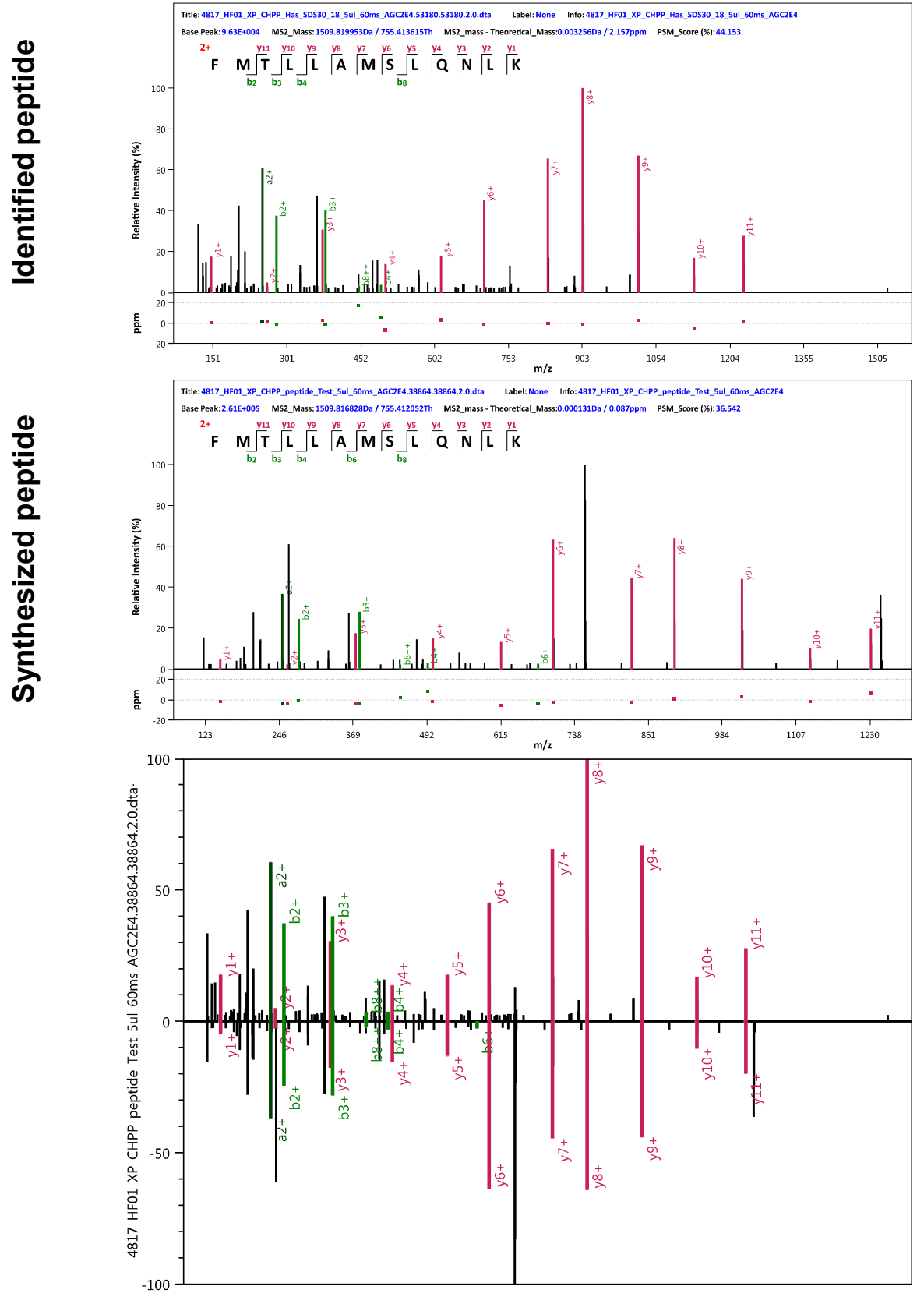

S-48 
Title: 4817_HF01_XP_CHPP_Has_Tricine20_10_5ul_60ms_AGC2E4.26905.26905.2.0.dta Label: None Info: 4817_HF01_XP_CHPP_Has_Tricine20_10_5ul_60ms_AGC2E4

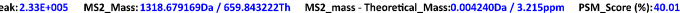

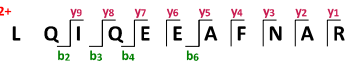

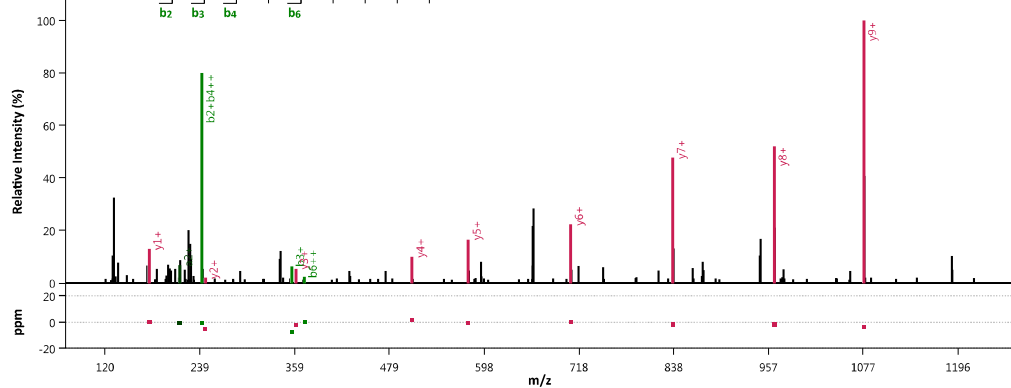

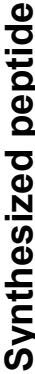

Title: 4817_HF01_XP_CHPP_peptide_Test_5ul_60ms_AGC2E4.20271.20271.2.0.dta Label: None Info: 4817_HF01_XP_CHPP_peptide_Test_Sul_60ms_AGC2EA

Base Peak: 5.26E+005 MS2_Mass: 1318.67625Da / 659.841763Th MS2_mass - Theoretical_Mass:0.001321Da/1.002ppm PSM_Score (\%): 36.536

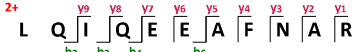
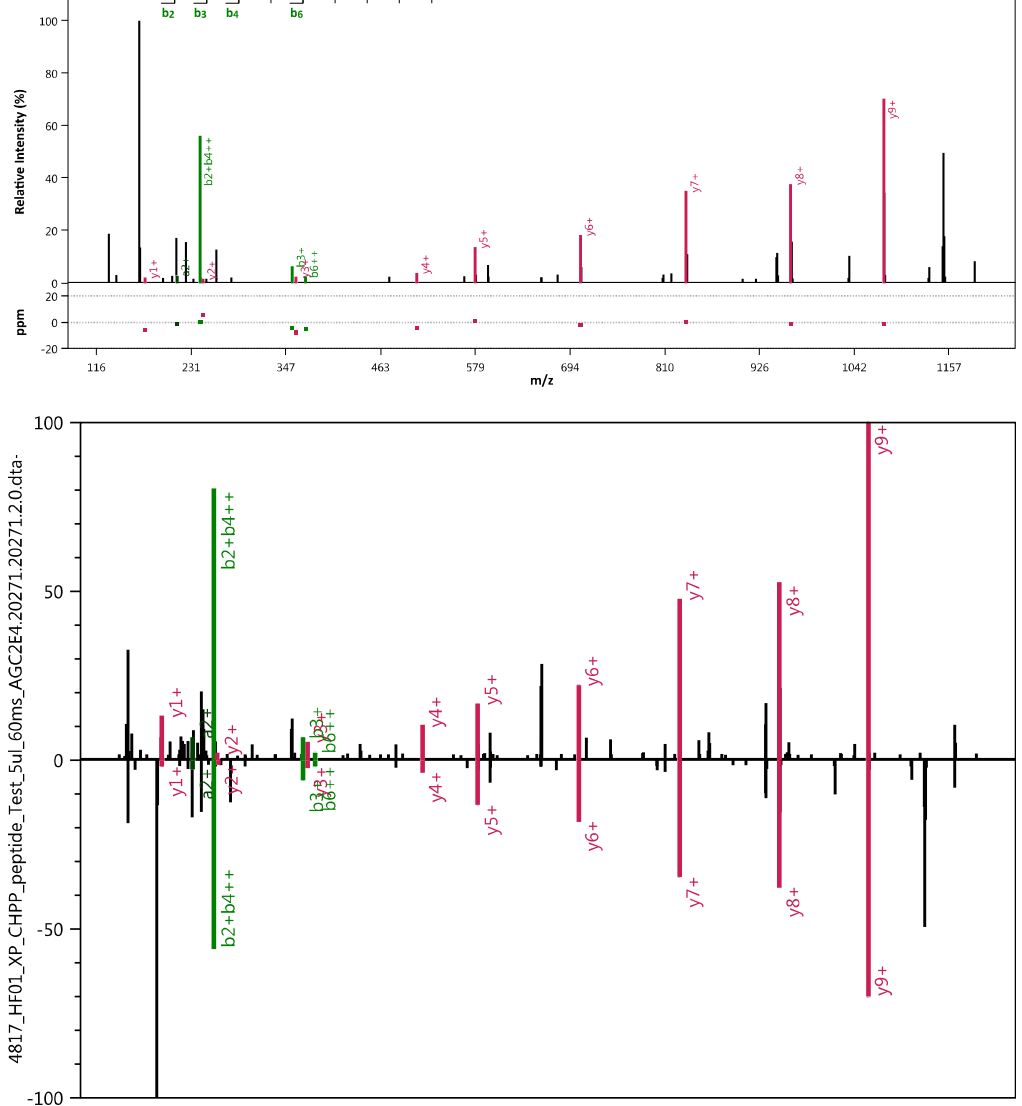


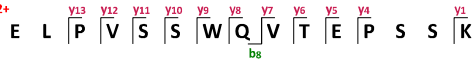

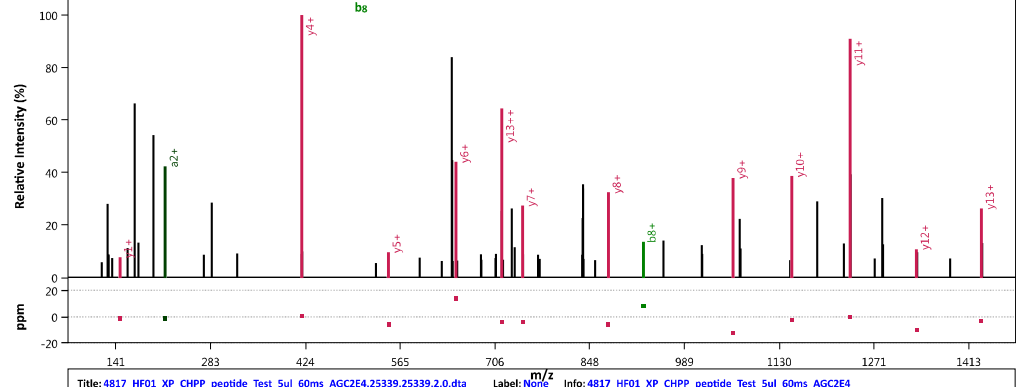

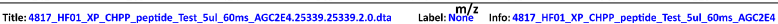
Base Peak: 1.01E+005 MS2_Mass: 1673.838324Da / 837.4228Th MS2_mass - Theoretical_Mass:0.000308Da / 0.184.ppm PSM_Score (\%): 43.719

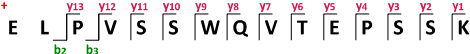

送
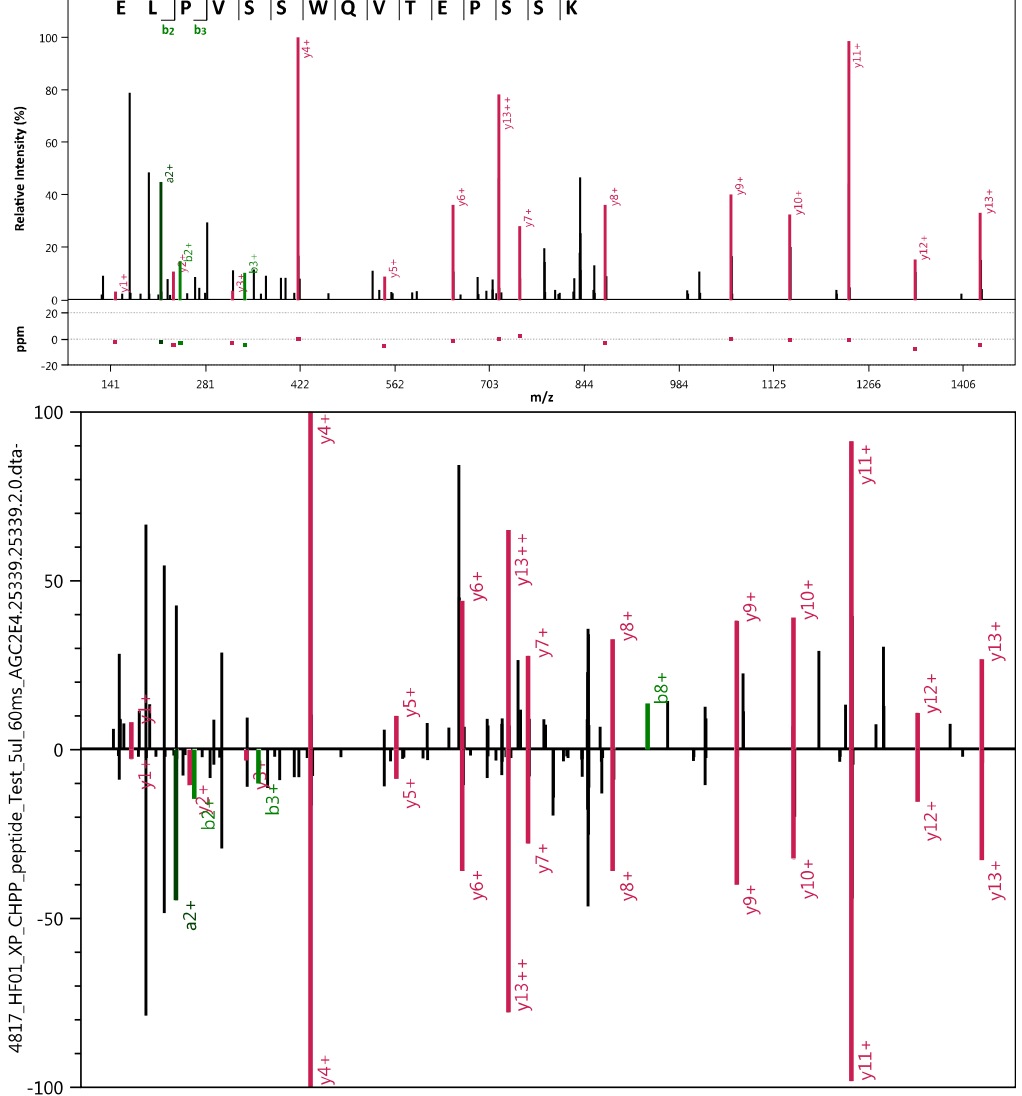
Title: 4817_HF01_XP_CHPP_Has_Tricine20_10_5ul_60ms_AGC2E4.9973.9973.3.0.dta Mods: 5,Carbamidomethy|[C]|Nonel; Label: None Info: 4817_HF01_XP_CHPP_Has_Tricine20_10_5ul_60ms Base Peak: 1.37E+005 MS2_Mass: 14855.715447Da / 495.91Th MS2_mass - Theorettcal_Mass:0.015294Da / 10.294ppm PSM_Score (\%): 14.248

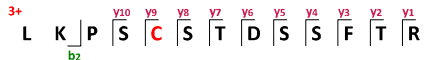
Base Peak: 1.02E+005 MS2_Mass: 1485.7000270a / 4955.90486Th MS2_mass - Theoretical_Mass:-0.000126Da /-0.085ppm PSM_Score (\%): 29.97

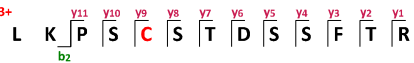
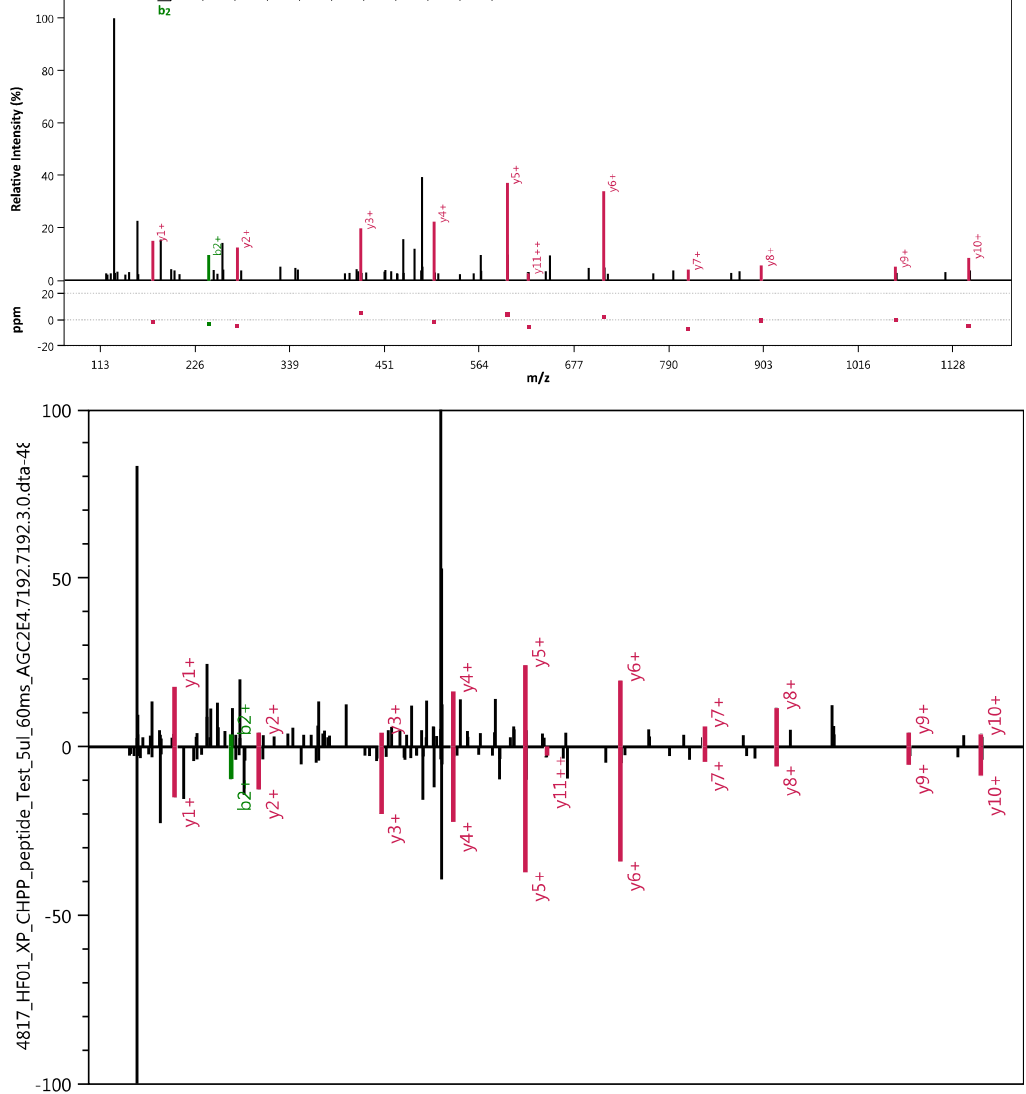


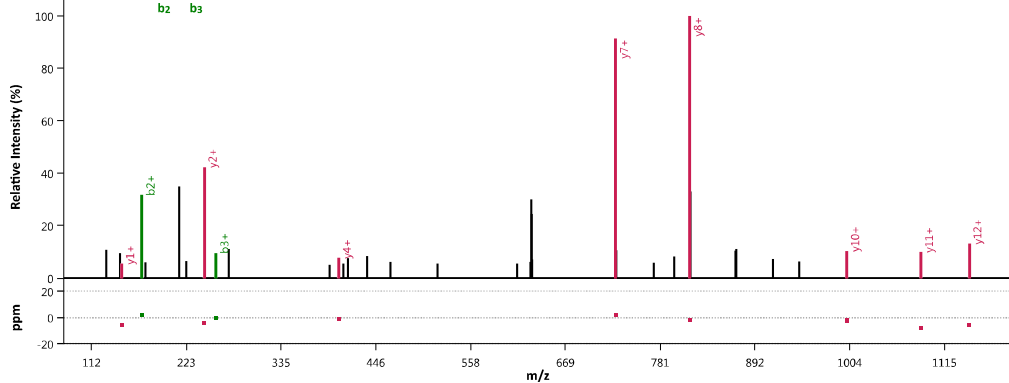

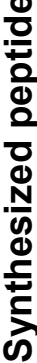

Fitle: 4817_HF01_XP_CHPP_peptide_Test_Sul_60ms_AGC2E4.3327.3327.2.0.dta Label: None Info: 4817_HF01_XP_CHPP_peptide_Test_5ul_60ms_AGC2E4

Base Peak:4.36E+007 MS2_Mass: 1257.644614Da/629.325945Th MS2_mass - Theoretical_Mass:0.001318Da/1.048ppm PSM_Score (\%):56.255

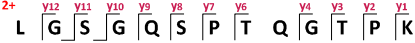
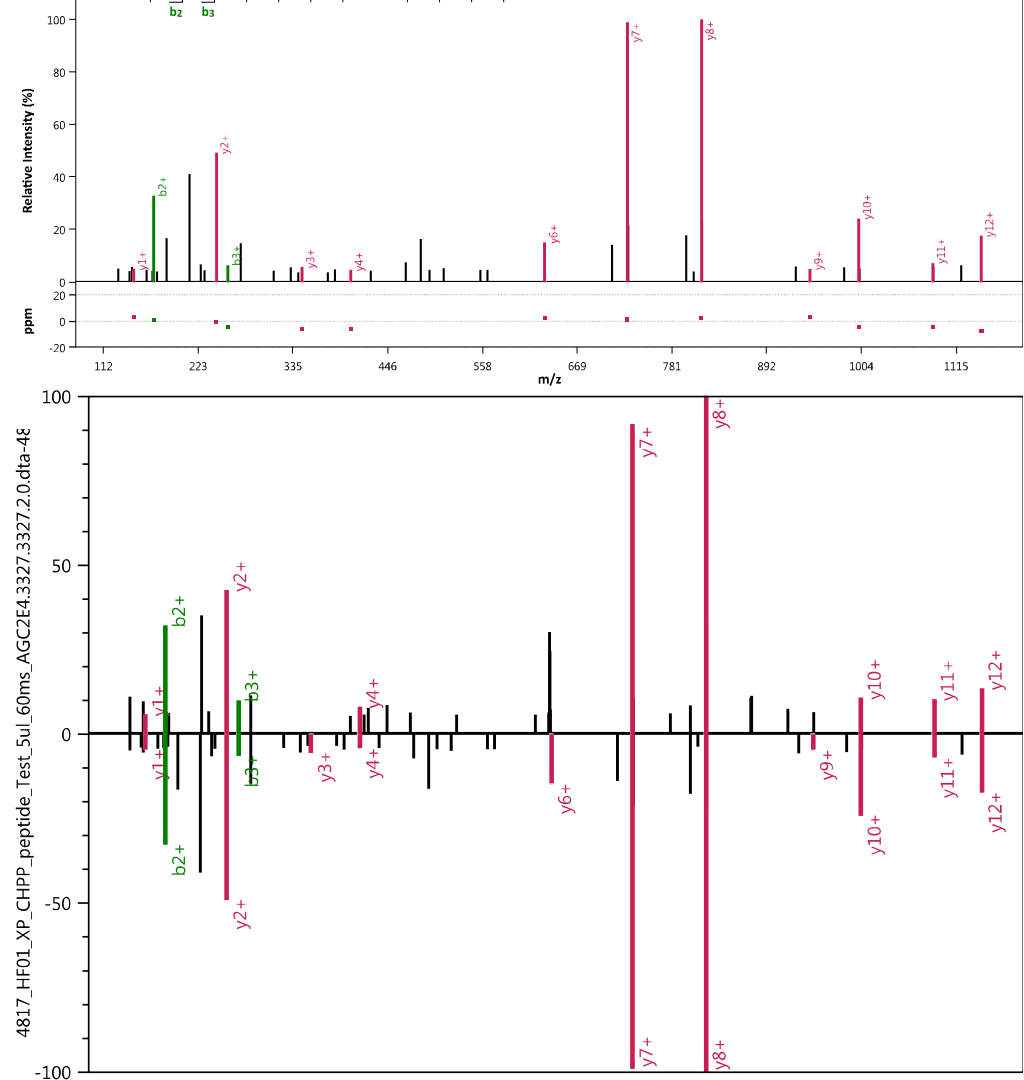


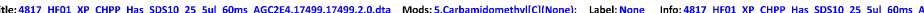
Base Peak: 1.75E+004 MS2_Mas5: 1381.666825Da / 691.337051Th MS2_mass - Theoretical_Mass:0.004117Da / 2.980ppm PSM_Score (\%):43.678

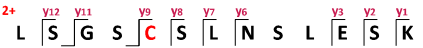

के

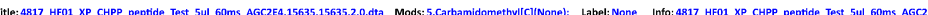
Base Peak:3.56E+005 MS2_Mass: 1381.664428Da / 691.335852Th MS2_mass - Theoretical_Mass:0.001720Da / 1.245ppm PSM_Score (ж): 69.499

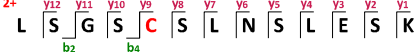
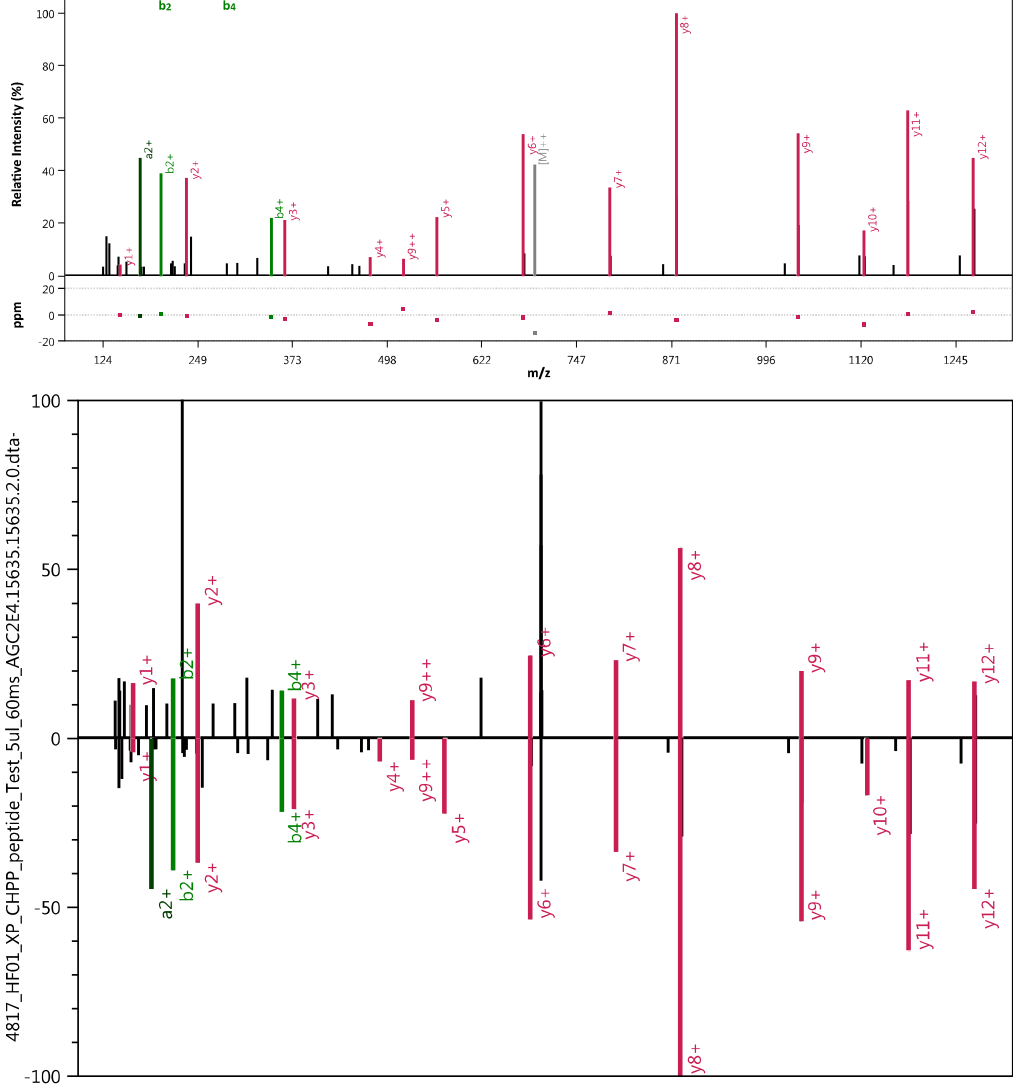


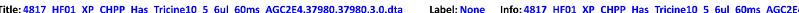
Base Peak:3.64E+004 MS2_Mass: 1090.711533Da/364.242029Th MS2_mass - Theoretical_Mass:0.002064Da/1.892ppm PSM_Score (\%): 54.38

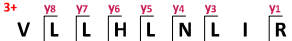

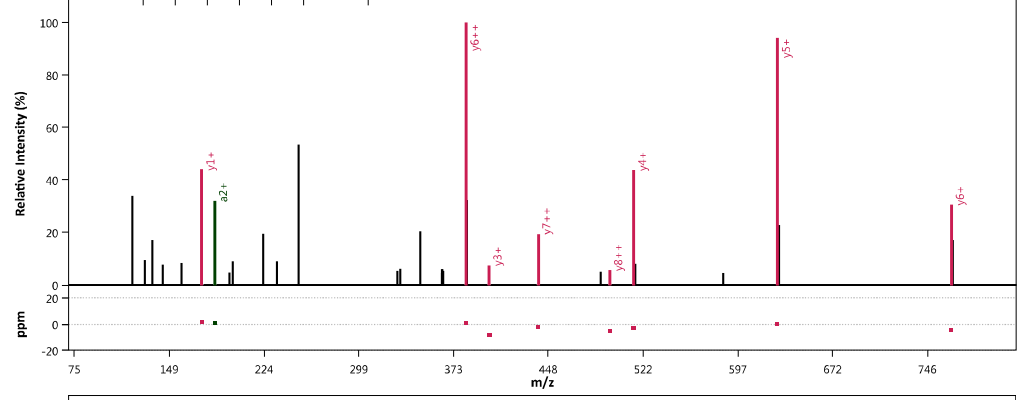

Titte:4817_HF01_XP_CHPP_peptide_Test_5ul_60ms_AGC2E4.28628.28628.3.0.dta Label: None Info: 4817_HF01_XP_CHPP_peptide__lest_5ul_60ms_AGC2E4 Base Peak: 1.18E+005 MS2_Mass: 1090.709905Da / 364.241486Th MS2_mass - Theoretical_Mass:0.000435Da/ 0.399ppm PSM__core (\%): 57.875

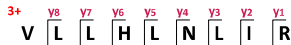

के
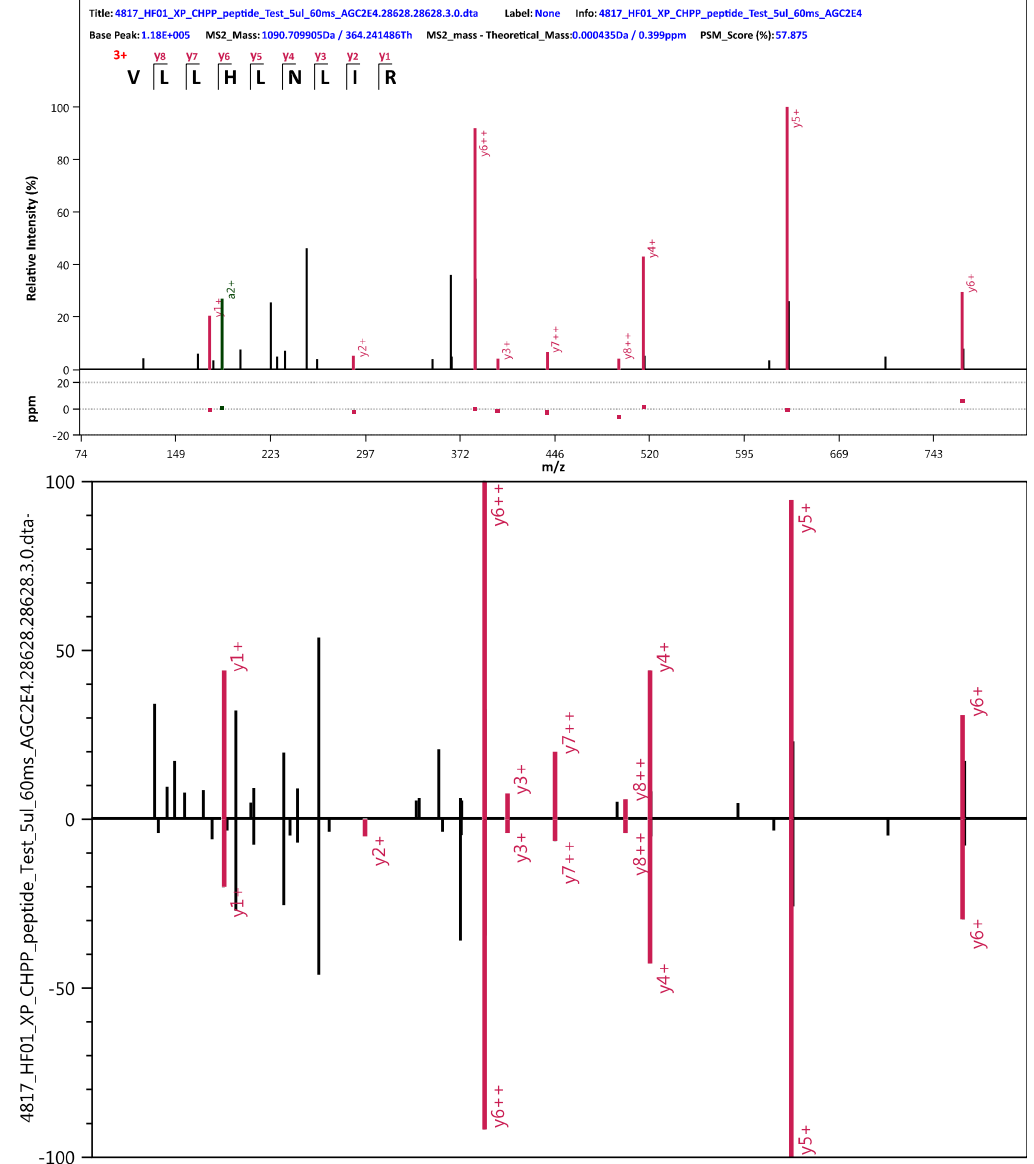
Title: 4817_HF01_XP_CHPP_Has_Tricine10_22_5ul_60ms_AGC2E4.14802.14802.2.0.dta Label: None Info: 4817_HF01_XP_CHPP_Has_Tricine10_22_5ul_60ms_AGCZEC Base Peak: 3.22E+005 MS2_Mass:937.427772Da / 469.217524Th MS2_mass - Theoretical_Mass:0.001661Da/1.772ppm PSM_Score (\%): 61.391

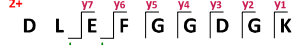

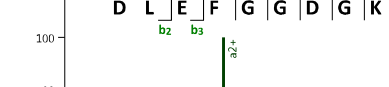

送

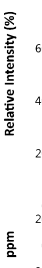

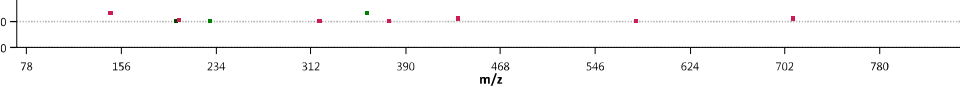

Title: 4817_HF01_XP_CHPP_peptide_Test_5ul_60ms_AGC2E4.13806.13806.2.0.dta Label: None Info: 4817_HF01_XP_CHPP_peptide_Test_5Ul_60ms_AGC2E4

Base Peak:2.24E+005 MS2_Mass:937.425962Da / 469.216619Th MS2_mass - Theoretical_Mass:-0.000150Da /-0.160ppm PSM_Score (\%): 58.422

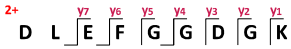
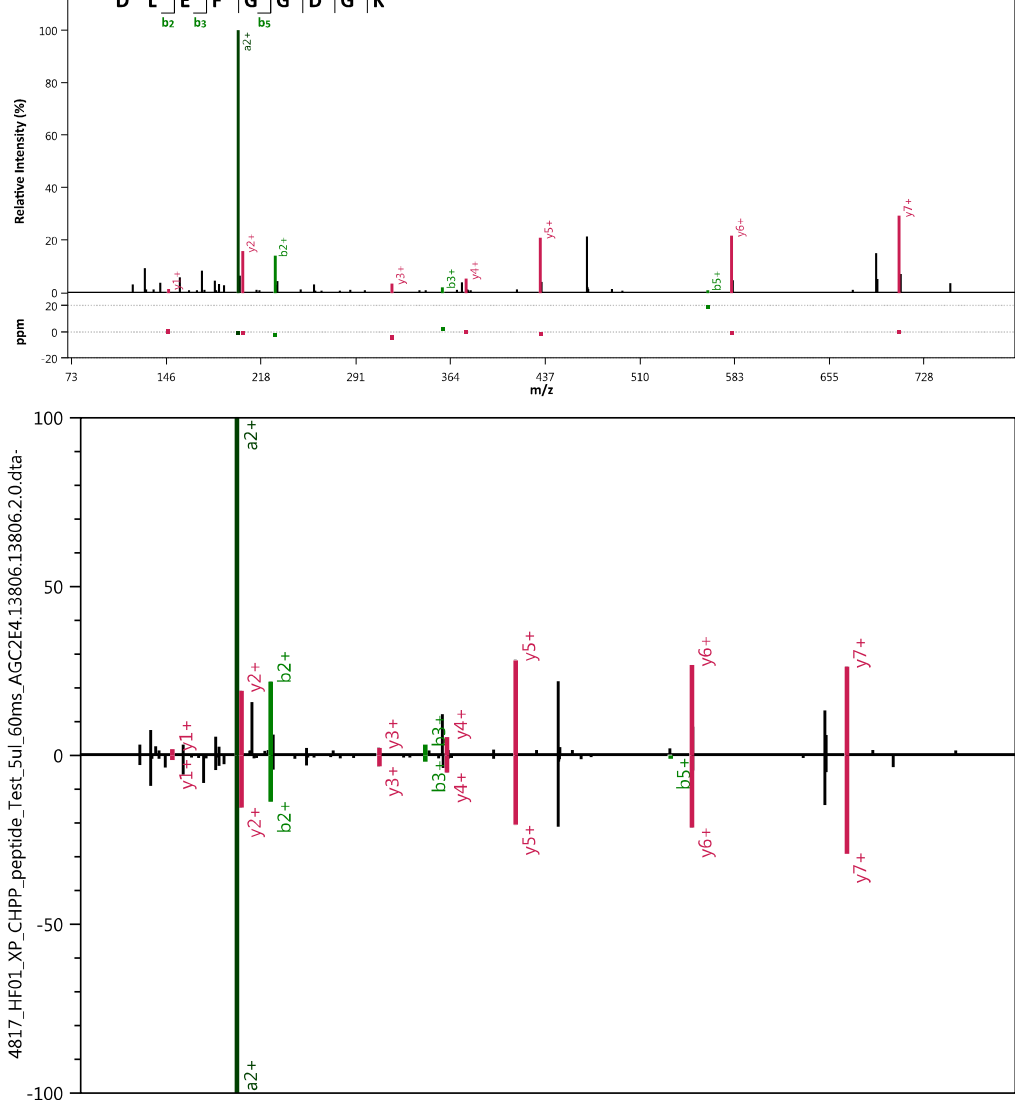
Title: 4817_HF01_XP_CHPP_Has__ricine10_22__5ul_60ms__AGC2E4.34935.34935.3.0.dta Label: None Info: 4817_HF01_XP_CHPP_Has_Tricine10_22_5ul_60ms_AGC2E4

Base Peak: 1.08E+005 MS2_Mass: 2046.162885Da / 682.725812Th MS2_mass - Theoretical_Mass:0.003617Da/1.768ppm PSM_Score (\%):36.425

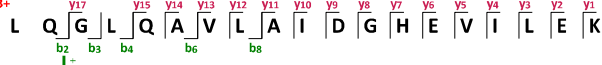

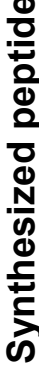

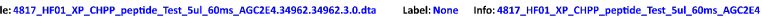

Base Peak: 7.59E+005 MS2_Mass:2046.162661Da / 682.725738Th MS2_mass-Theoretical_Mass:0.003394Da / 1.659ppm PSM_Score (\%);:42.313

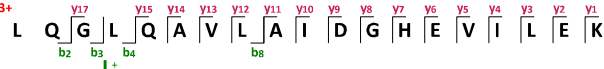
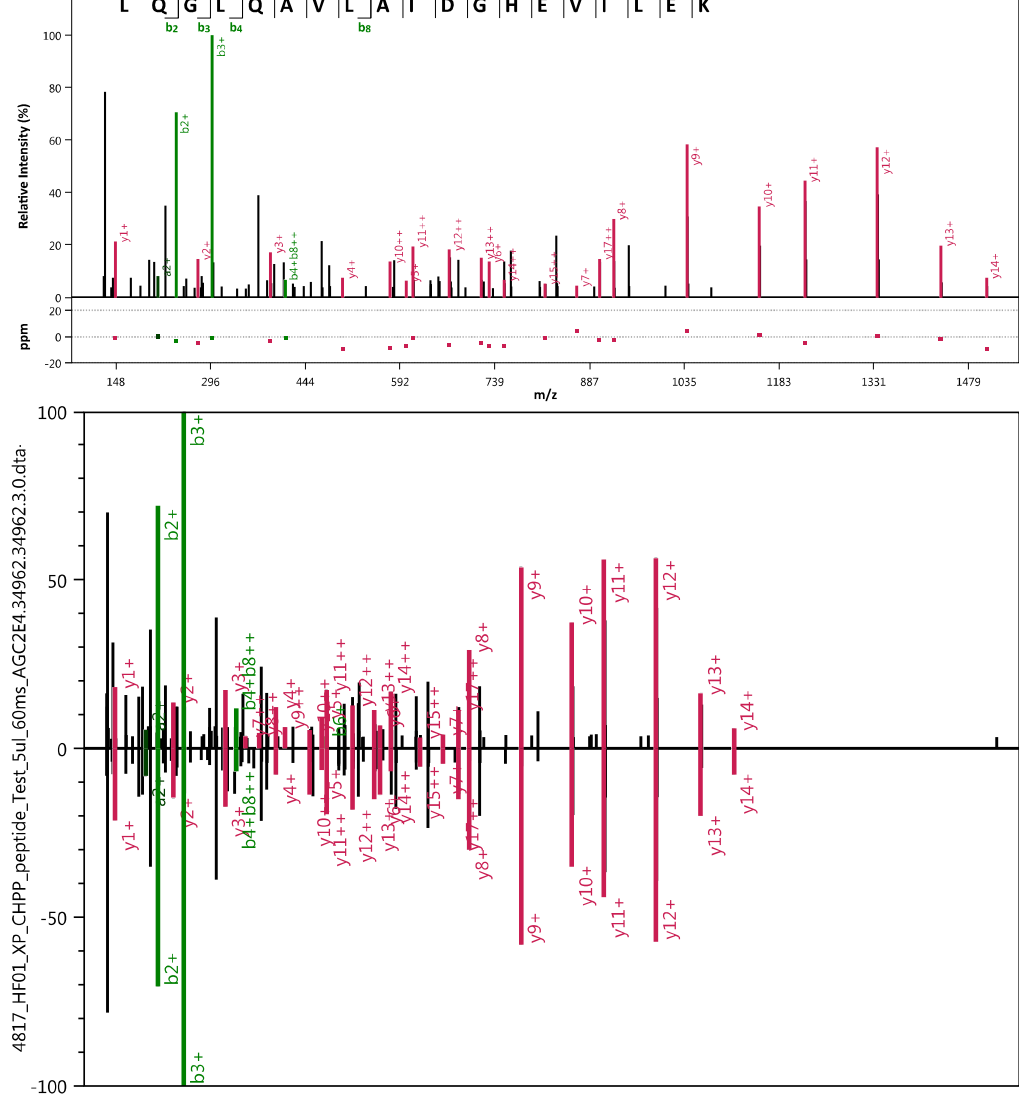
Title:4817_HF01_XP_CHPP_Has__SDS10_17_5ul_60m5_AGC2E4.22412.22412.2.0.dta Label: None Info; 4817_HF01_XP_CHPP_Has_SOS10_17_5ul_60ms_AGC2

Base Peak:9.08E+004 MS2_Mass: 1477.732724Da / 739.37Th MS2_mass - Theoretical_Mass:0.004644Da/3.142ppm PSM_Score (\%): 45.477

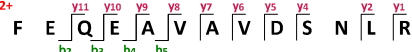

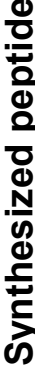

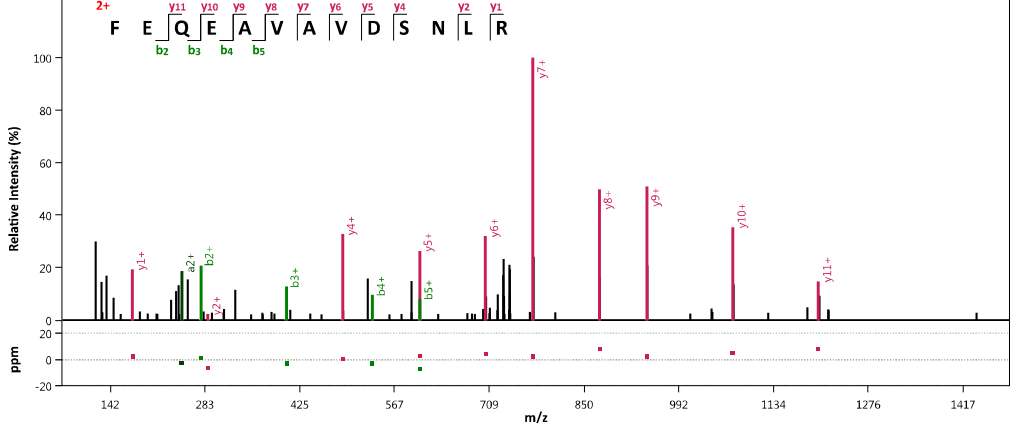
Base Peak: 2.38E +005 MS2_Mass: 1477.73045Da / 739.368863Th MS2_mass - Theoretical_Mass:0.0023700a / 1.604ppm PSM_Score (\%): 48.691

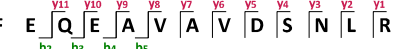
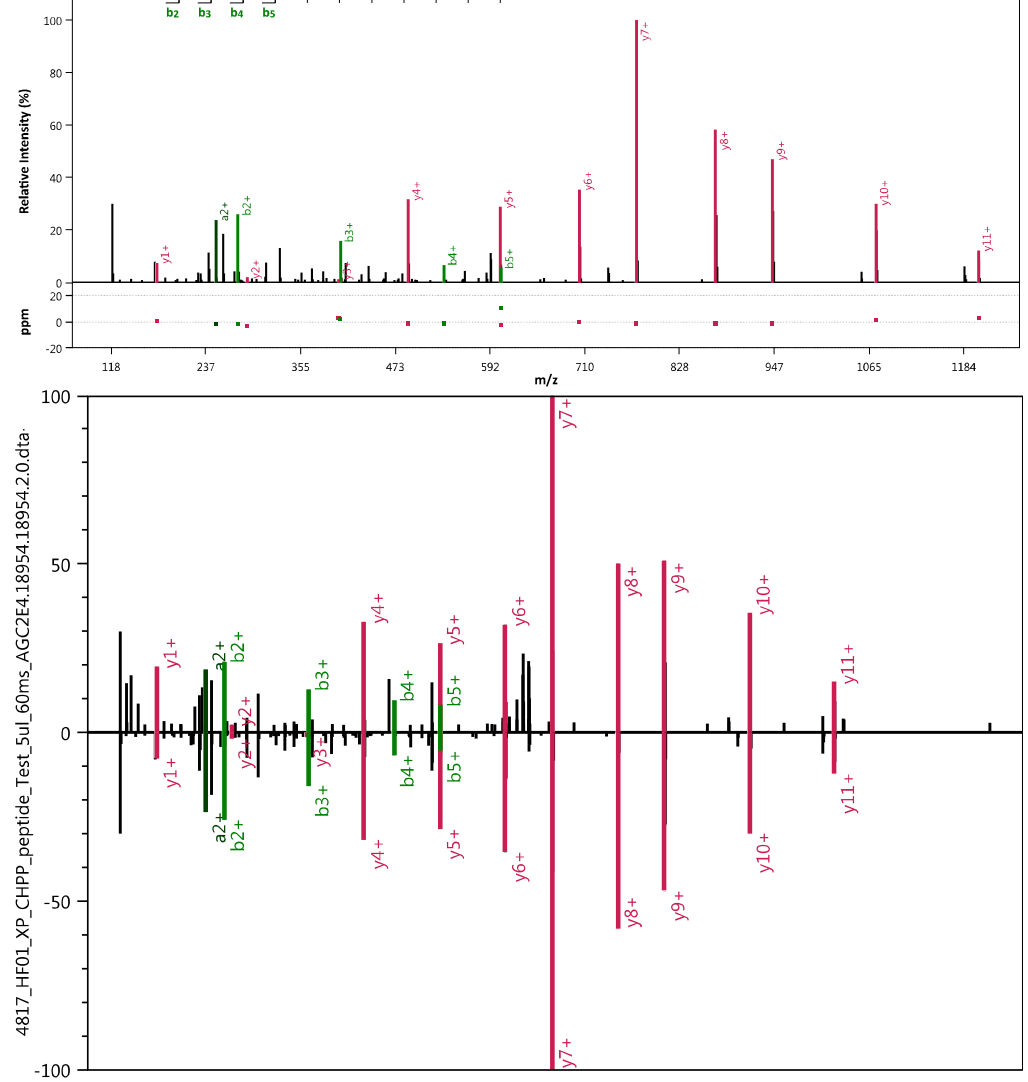
Title: 4817_HF01_XP_CHPP_Has_Tricine10_8_5ul_60ms_AGC2E4.33347.33347.3.0.dta Label: None Info: 4817_HF01_XP_CHPP_Has_Tricine10_8_5ul_60ms__AGC2E4

Base Peak: 9.55E+004 MS2_Mass: 1908.960427Da/636.99166Th MS2_mass - Theoretical_Mass:0.005603Da / 2.935ppm PSM_Score (\%): 34.532

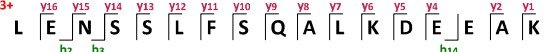
ase Peak: 1.83E+005 MS2_Mass: 1908.958708Da / 636.991087Th MS2_mass - Theoretical_Mass:0.0038840a / 2.035ppm PSM_SCore (\%): 42.79

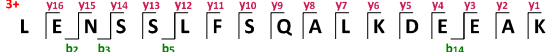
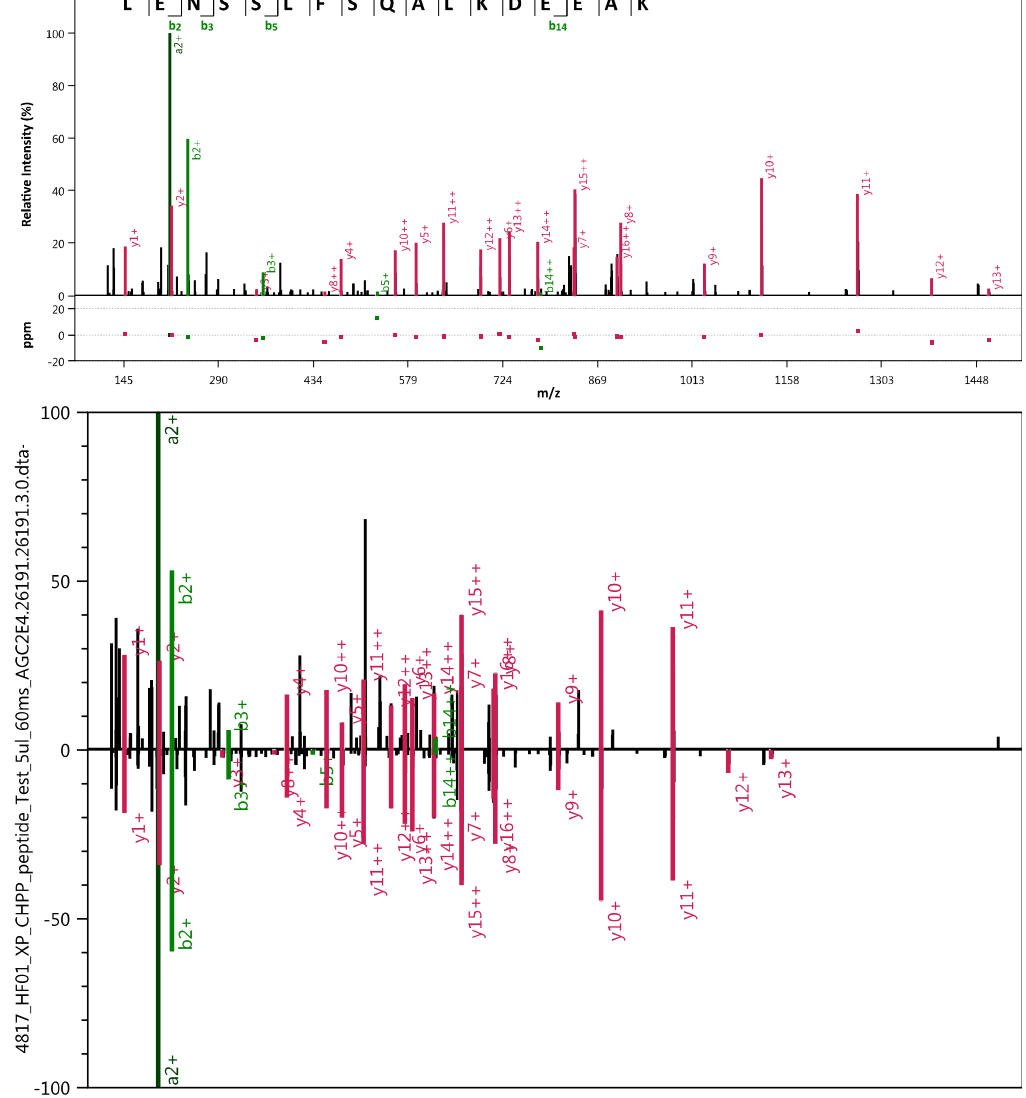
Title: 4817_HF01_XP_CHPP_Has_SDS10_17_5ul_60ms_AGC2E4.31042.31042.3.0.dta Label: None Info: 4817_HF01_XP_CHPP_Has_SDS10_17_5ul_60m5_AGC2E4

源

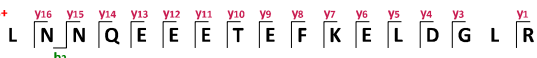

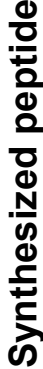

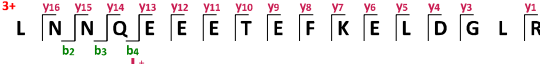
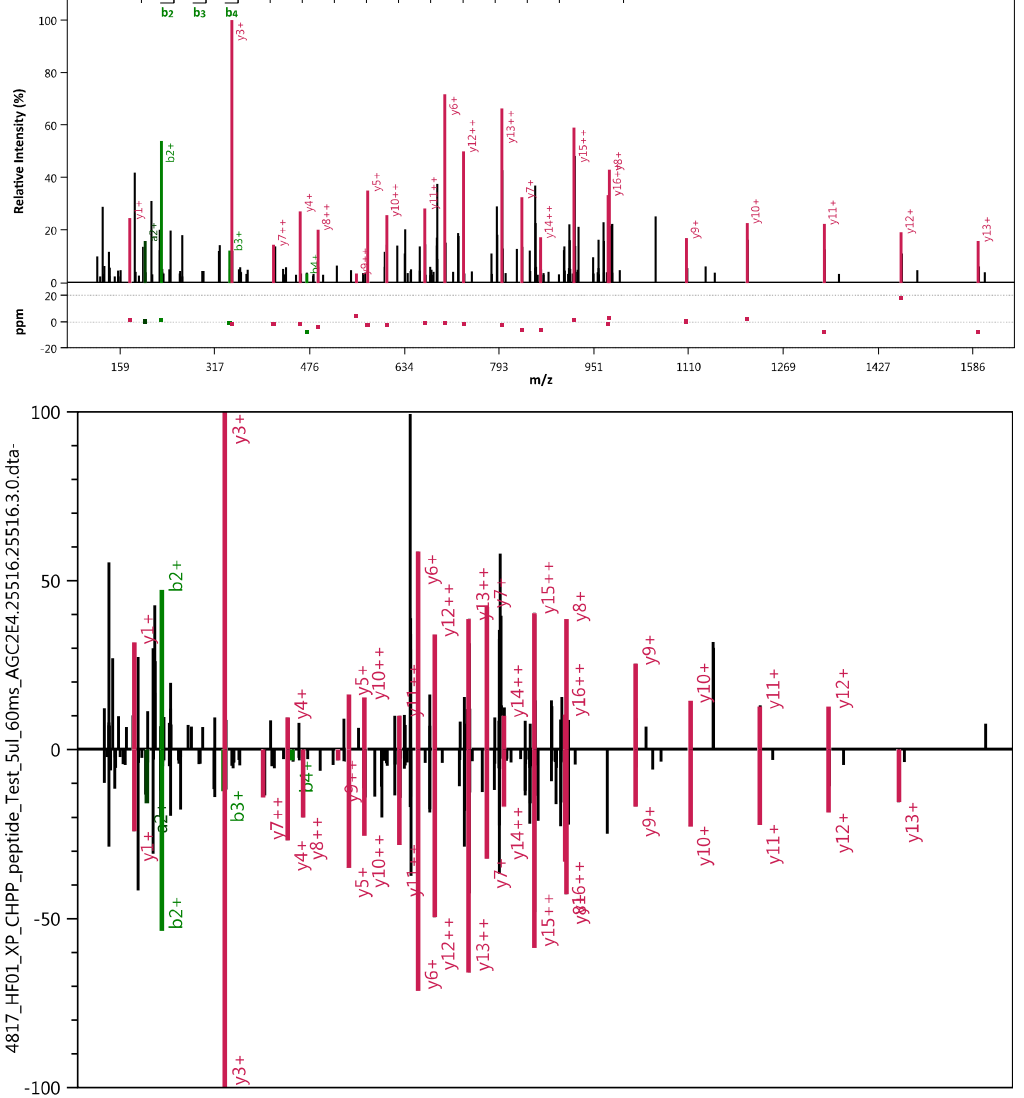


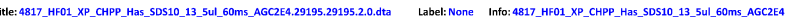
Dase Peak: 3.68E+005 MS2_Mass: 1502.715163Da/751.86122Th MS2_mass - Theoretical_Mas5:0.003067Da/2.041ppm PSM_Score (\%):28.185

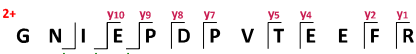

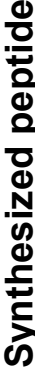

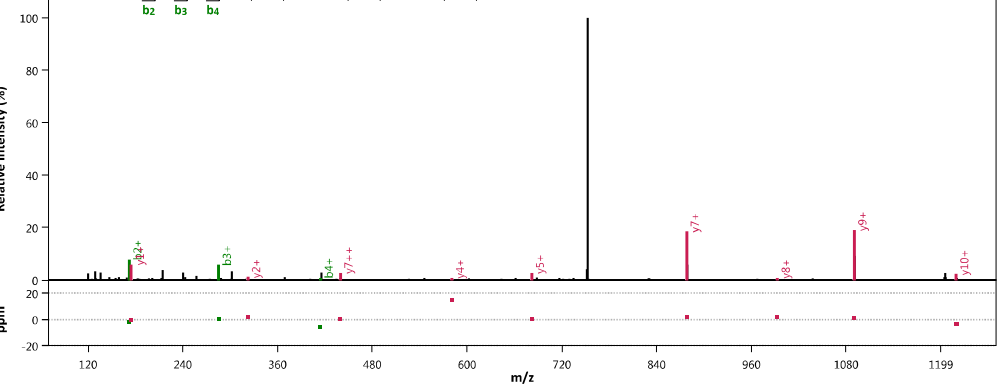

Title: 4817_HF01_XP_CHPP__eptide_Test_SUl_600ms_AGC2E4.25185.25185.2.0.dta Label: None Info: 4817_HF01_XP_CHPP_peptide_Test_5ul_60ms_AGC2E4

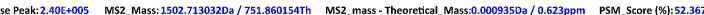

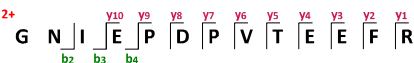
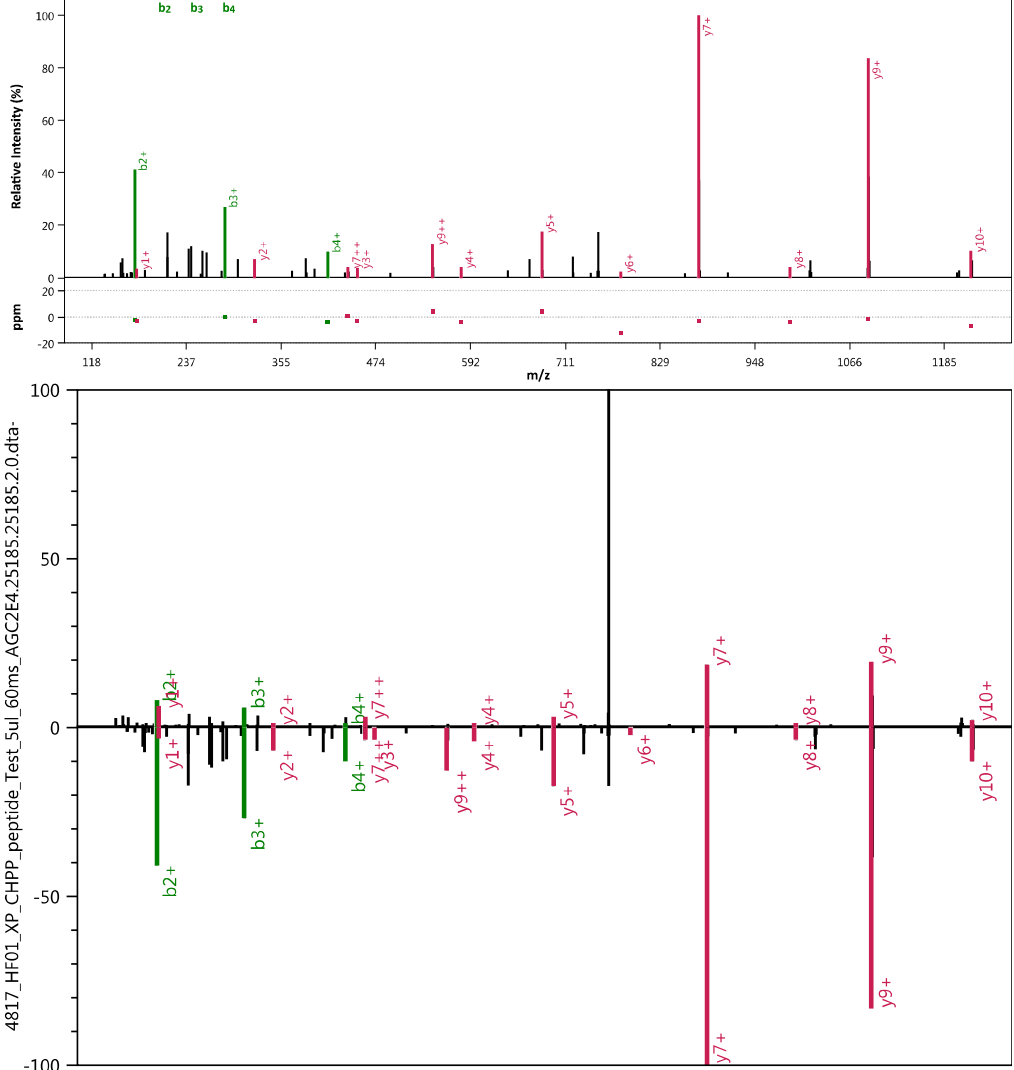

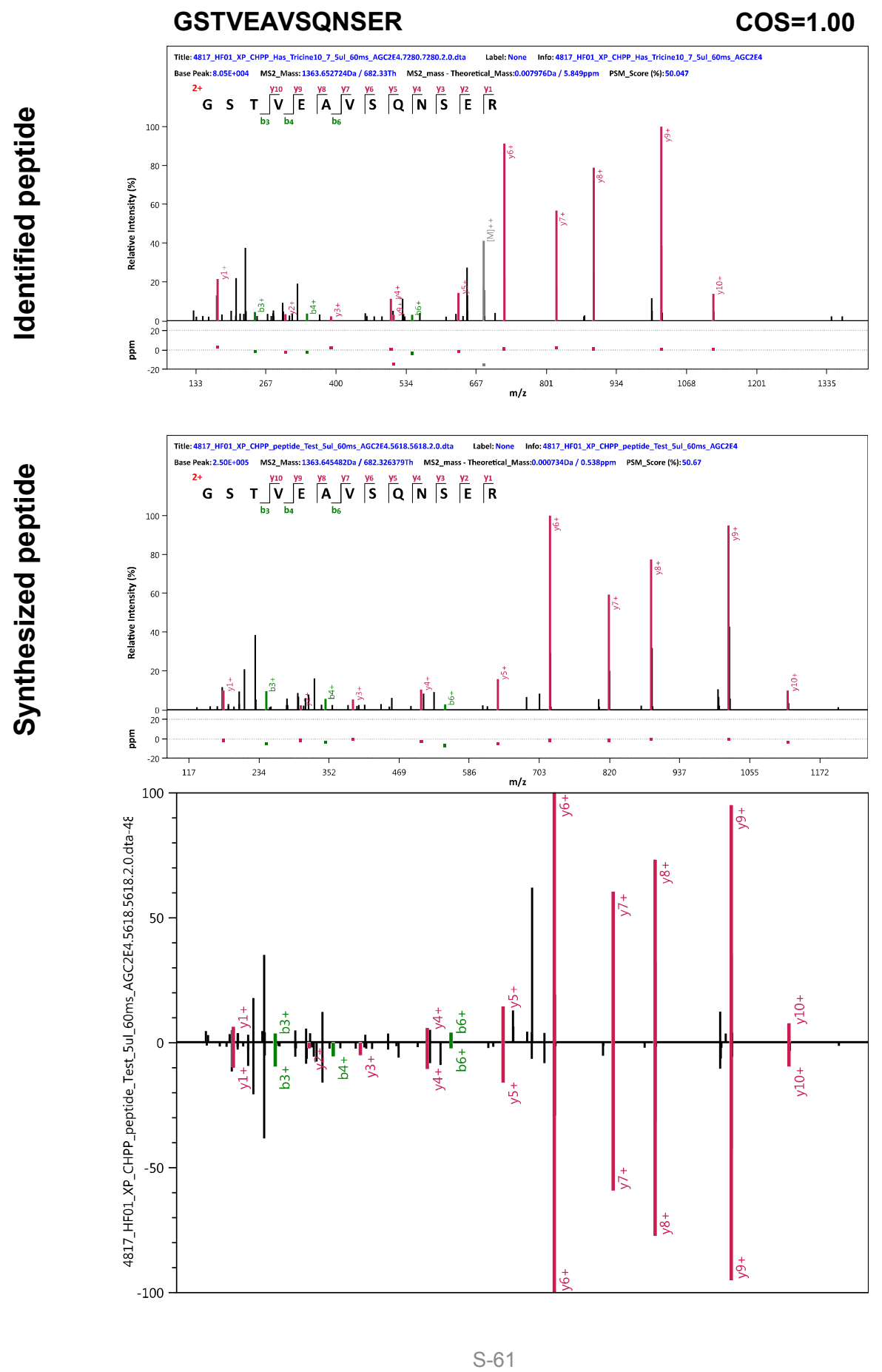


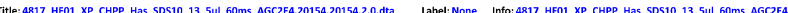
Base Peak: 1.12E+005 MS2_Mass: 1433.733961Da/ 717.370619Th MS2_mass - Theoretical_Mass:0.003573Da/2.492ppm PSM_Score (x):29.48

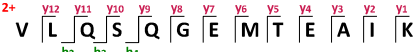

के

Title: 4817_HF01_XP_CHPP_peptide_Test_Sul_60m5_AGC2E4.15805.15805.2.0.dta Label: None Info: 4817_HF01_XP_CHPP_peptide_Test_Sul_60ms_AGC2E4

作

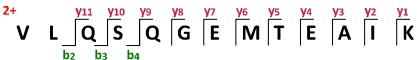
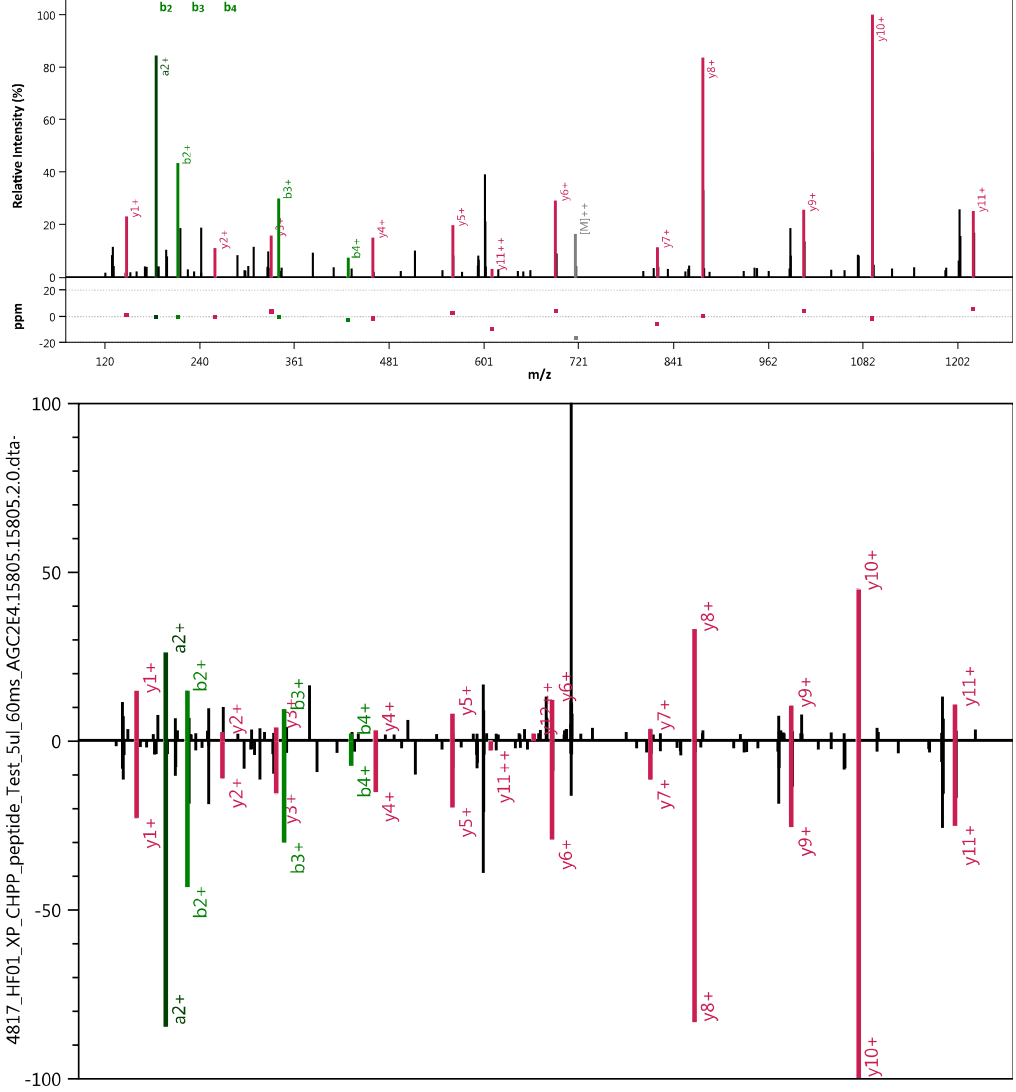

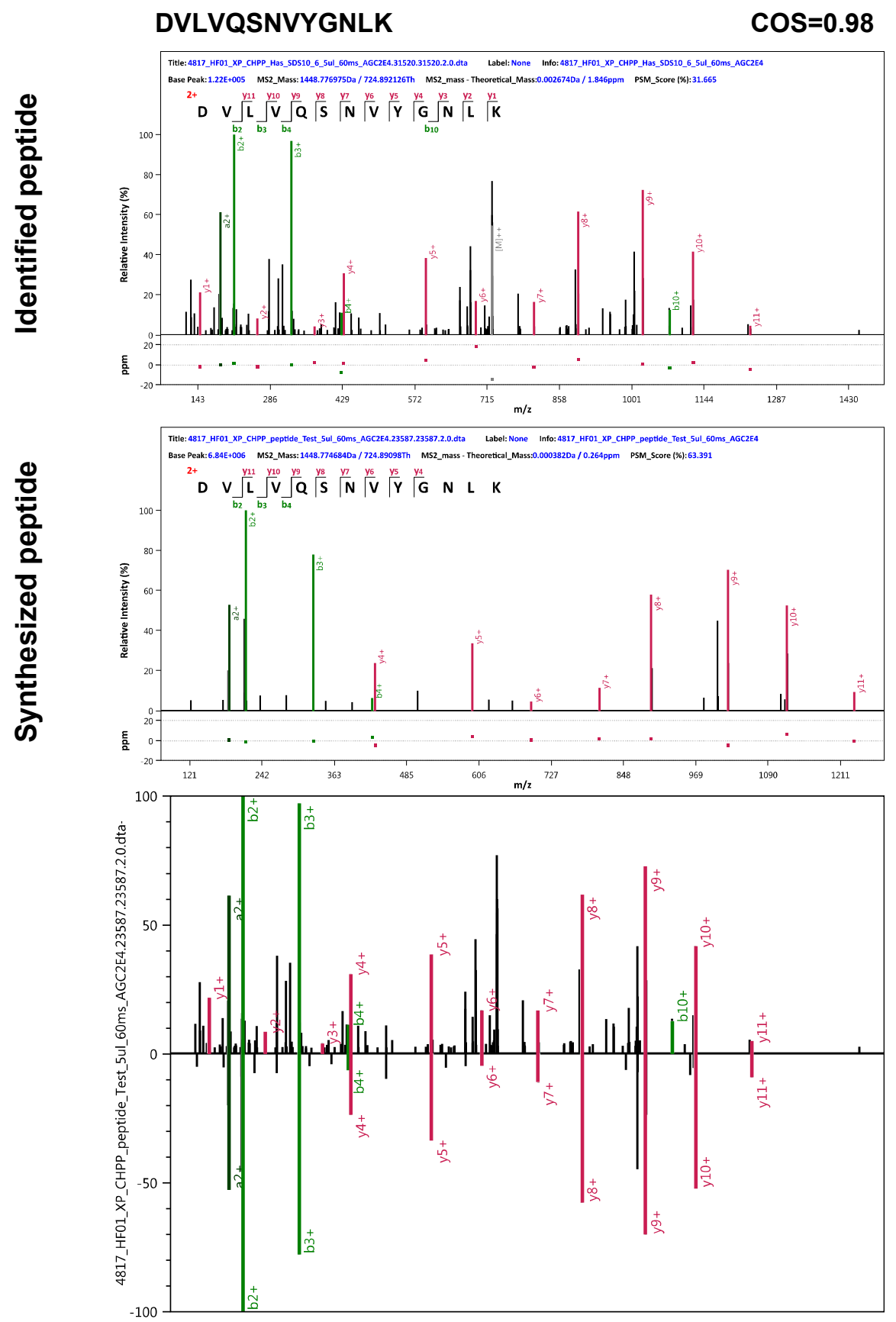

S-63 


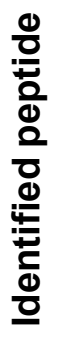

Title: 4817_HF01_XP_CHPP_Has_Tricine30_2__Sul_60ms_AGC2E4.10731.10731.2.0.dta Label: None Info: 4817_HF01_XP_CHPP_Has_Tricine30_2_Sul_60ms_AGC2E4

Base Peak:4.99E+004 MS2_Mass: 1594.72156Da / 797.864418Th MS2_mass - Theoretical_Mass:0.009061Da / 5.682ppm PSM_Score (\%): 30.262

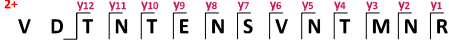

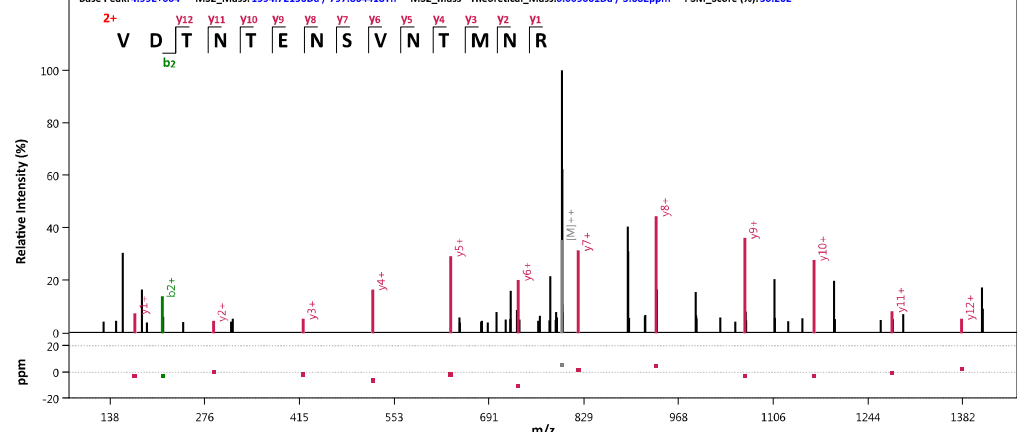

$691 \mathrm{~m} / 2$

Title: 4817_HF01_XP_CHPP_peptide__Test_5ul_60ms_AGC2E4.7962.7962.2.0.dta Label: None Info: 4817_HF01_XP_CHPP_peptide_Test_5ul_60ms_AGC2E4

Base Peak: 1.06E+005 MS2_Mass: 1594.713162Da / 797.860219Th MS2_mass - Theoretical_Mass:0.000662Da / 0.415ppm PSM_Score (\%): 59.431

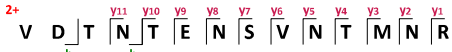

के
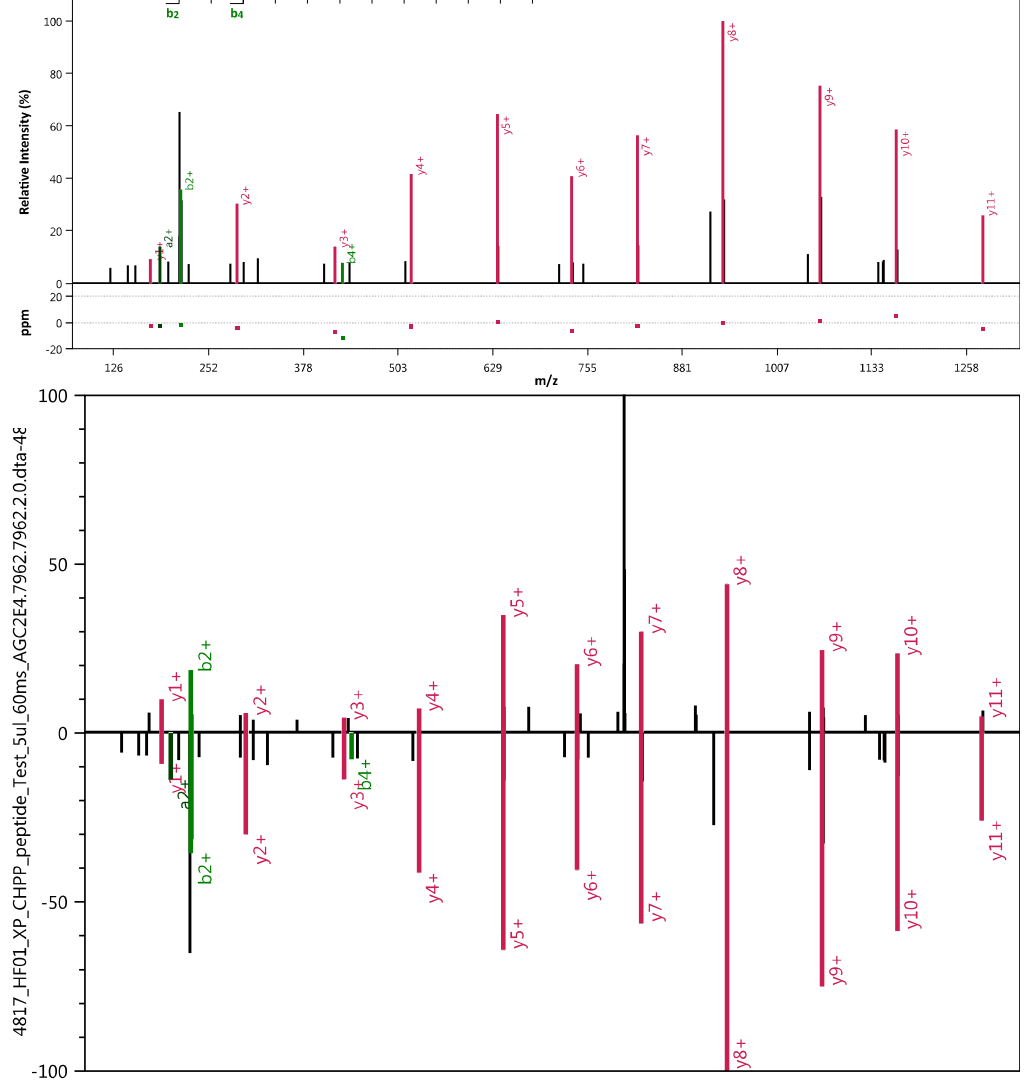


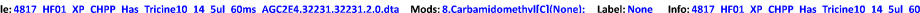
Base Peak:3.90E+004 MS2_Mass: 1922.87365Da / 961.940463Th MS2_mass - Theoretical_Mass:0.003595Da / 1.869ppm PSM_Score (\%): 58.306

${ }^{2+}$ A A A D D D

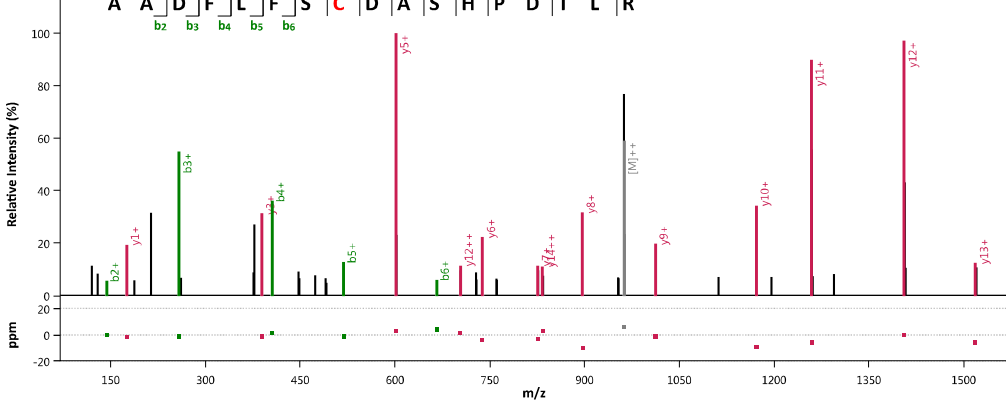

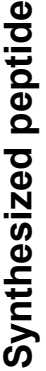

Title: 4817_HF01_XP_CHPP_peptide_Test_5ul_60ms_AGC2E4.26813.26813.2.0.dta Mods: 8,Carbamidomethyl[CII(None); Label: None Info: 4817_HF01_XP_CHPP_peptide_Test_5ul_60ms_AGC2 Base Peak:2.89E+005 MS2_Mass: 1922.87071Da / 961.938993Th MS2_mass - Theoretical_Mass:0.000655Da / 0.340ppm PSM_Score (\%): 50.318

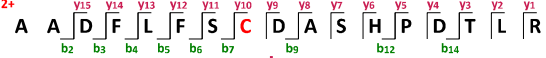
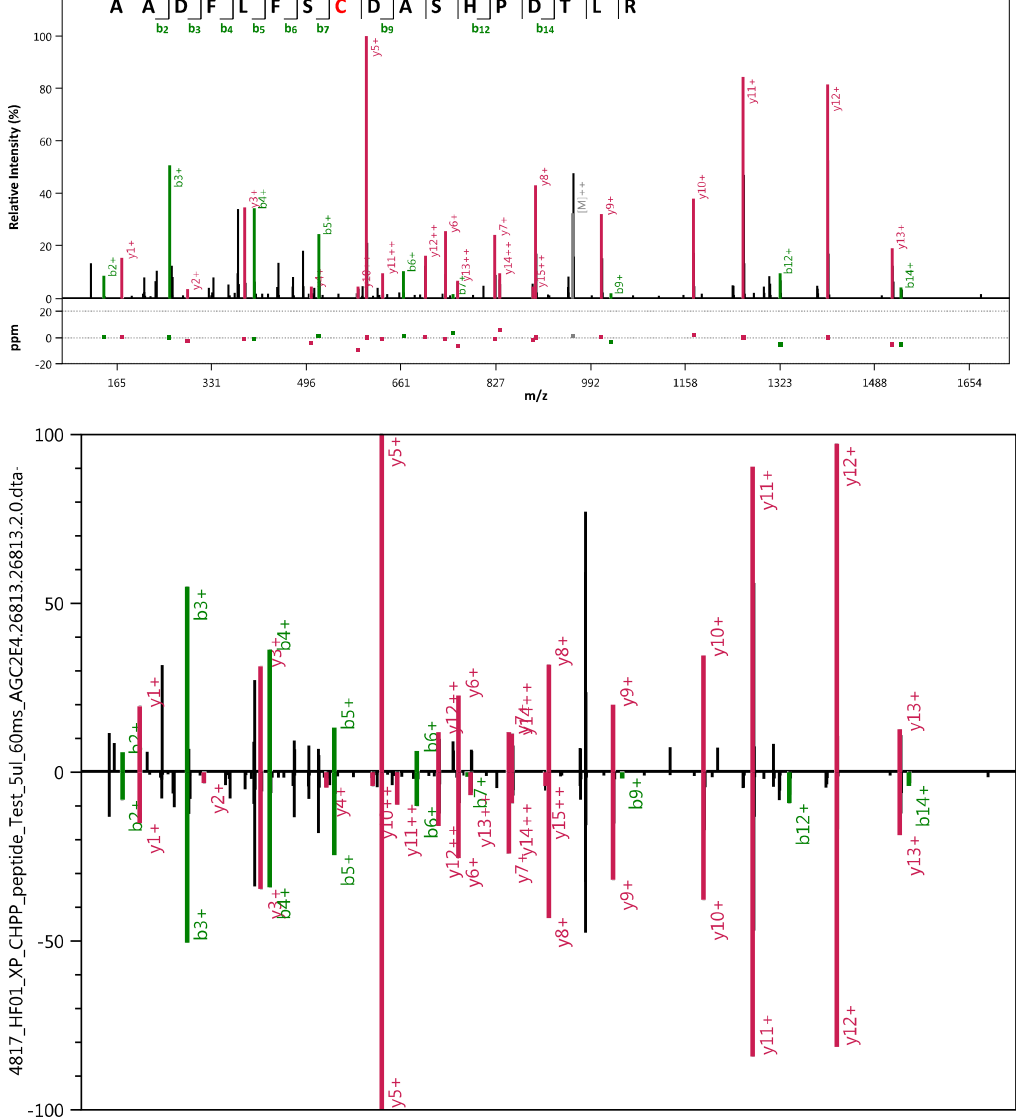
Title: 4817_HF01_XP_CHPP_Has_Tricine20_14_5ul_60ms_AGC2E4.32562.32562.20.dta Mods: 4,Carbamidomethyl[C][None); Label: None Info: 4817_HF01_XP_CHPP_Has_Tricine20_14_5ul_60 Base Peak: 1.03E+005 MS2_Mass: 1411.652724Da/706.33Th MS2_mass - Theoretical_Mass:0.010473Da / 7.419ppm PSM_Score (\%): 51.664

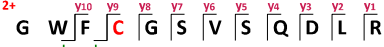

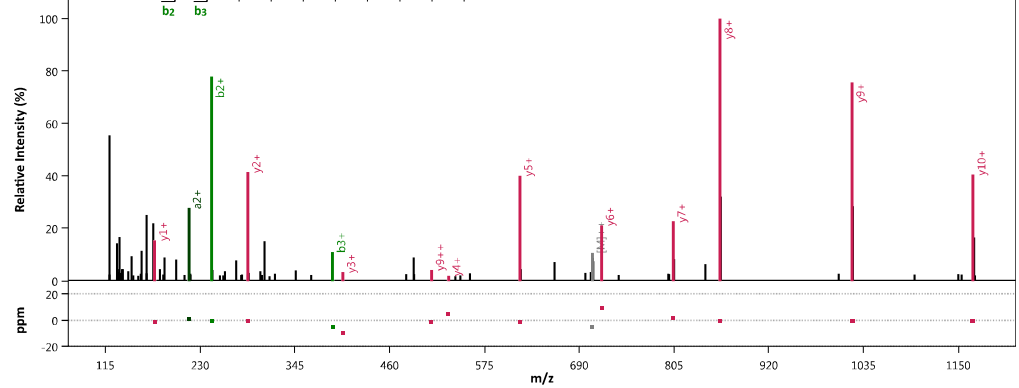

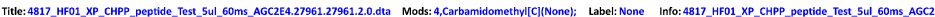
Base Peak: 1.71E+006 MS2_Mass: 1411.645436Da / 706.326356Th MS2_mass - Theoretical_Mass:0.003185Da / 2.256ppm PSM_Score (6): 59.048

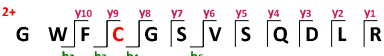

के
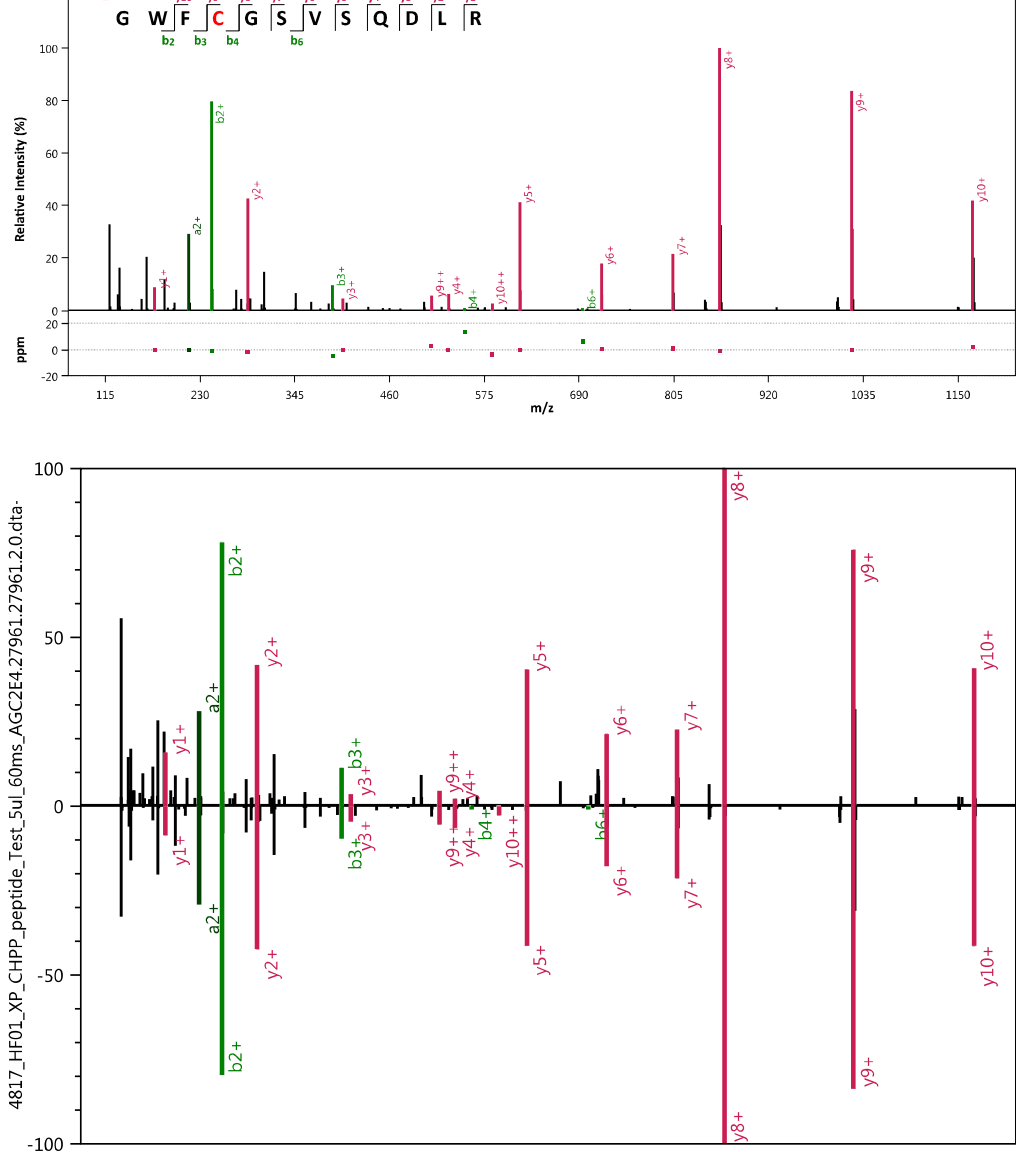


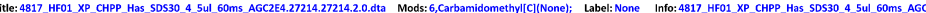
tase Peak: 1.20Et+005 MS2_Mass: 1396.657890a / 698.832583Th MS2_mass - Theoretical_Mass:0.005411Da / 3.874ppm PSM_Score (\%):40.078

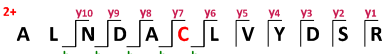

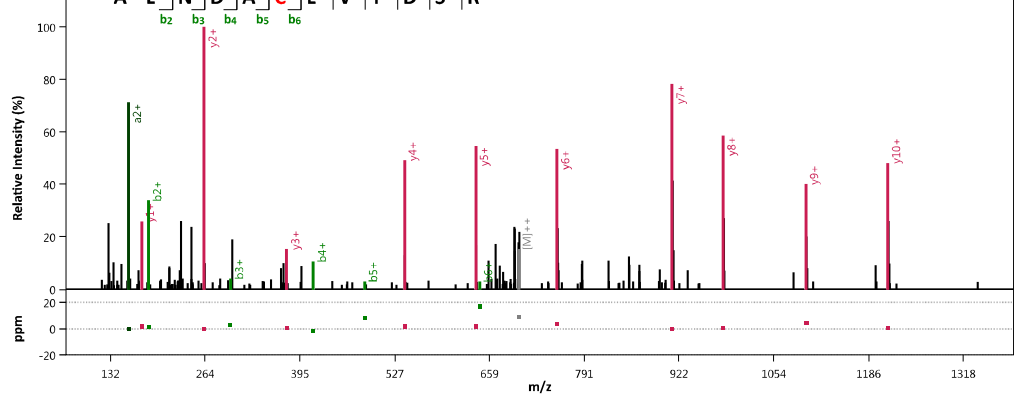

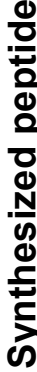

作 Base Peak: 1.75E+005 MS2_Mass: 1396.652970a / 698.830123Th MS2_mass - Theoretical_Mass:0.000490Da/0.351ppm PSM_Score (\%): 56.743

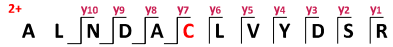
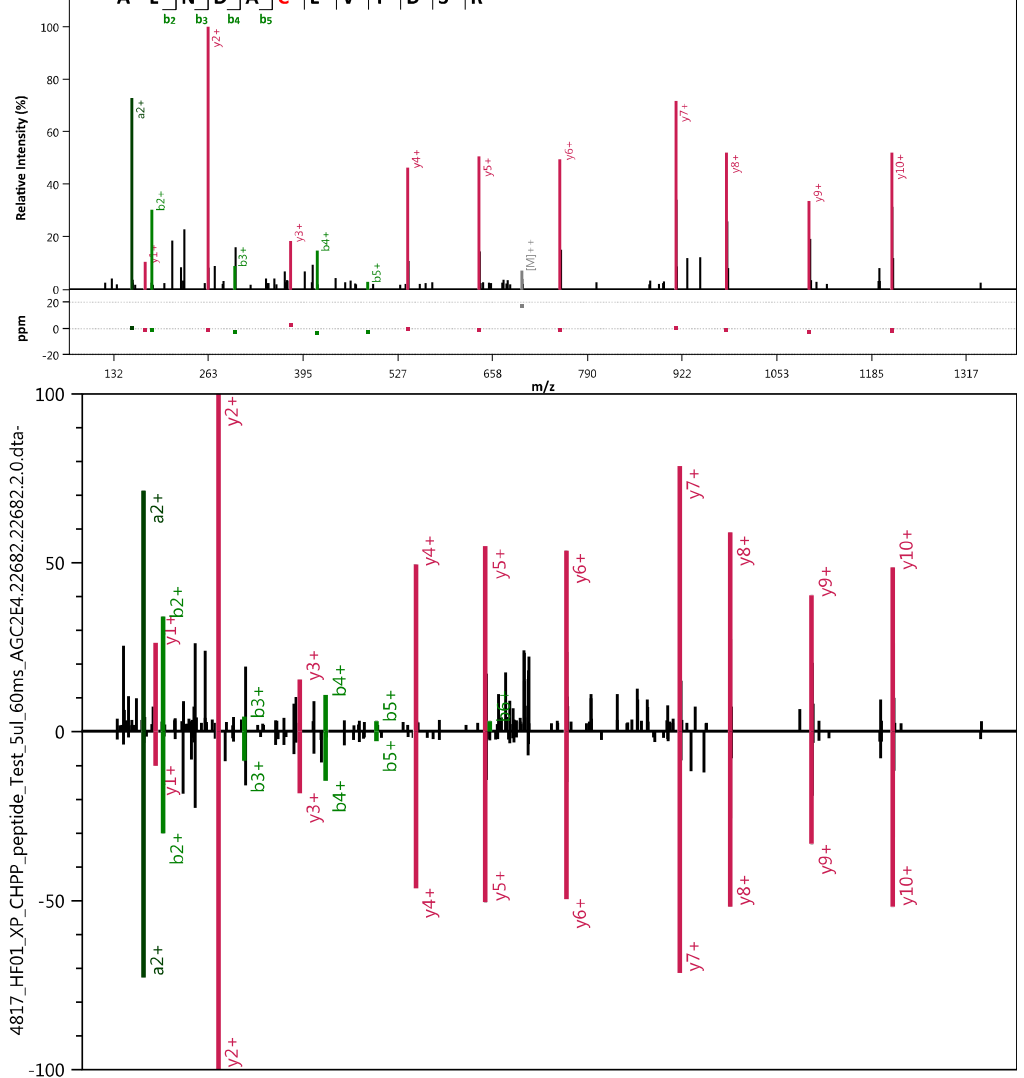


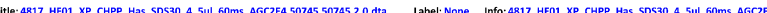
Base Peak: 4.68E+004 MS2_Mass: 1118.685952Da / 559.846614Th MS2_mass - Theoretical_Mass:0.004032Da/ 3.605ppm PSM_Score (\%):30.743

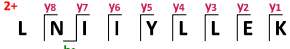

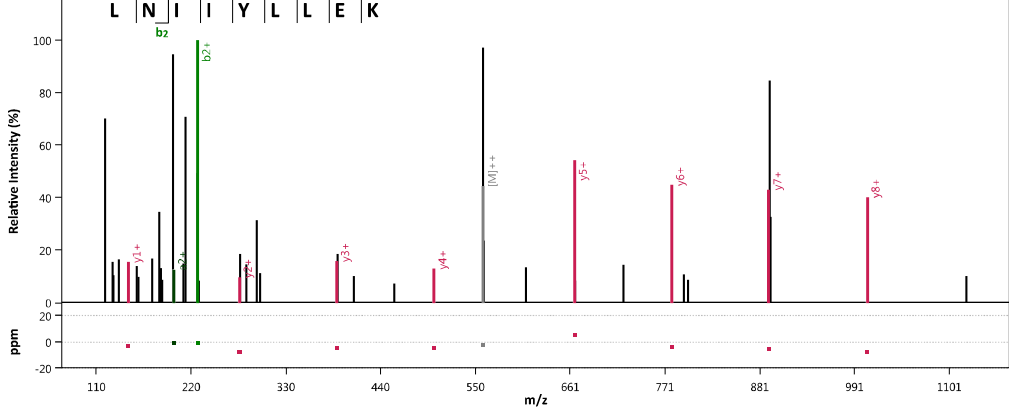

을

Tittle: 4817_HF01_XP_CHPP_peptide_Test_5ul_60ms_AGC2E4.36872.36872.2.0.dta Label: None Info: 4817_HF01_XP_CHPP_peptide_Test_5ul_60ms_AGC2E4

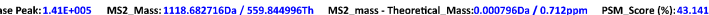

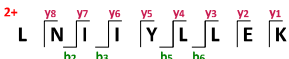
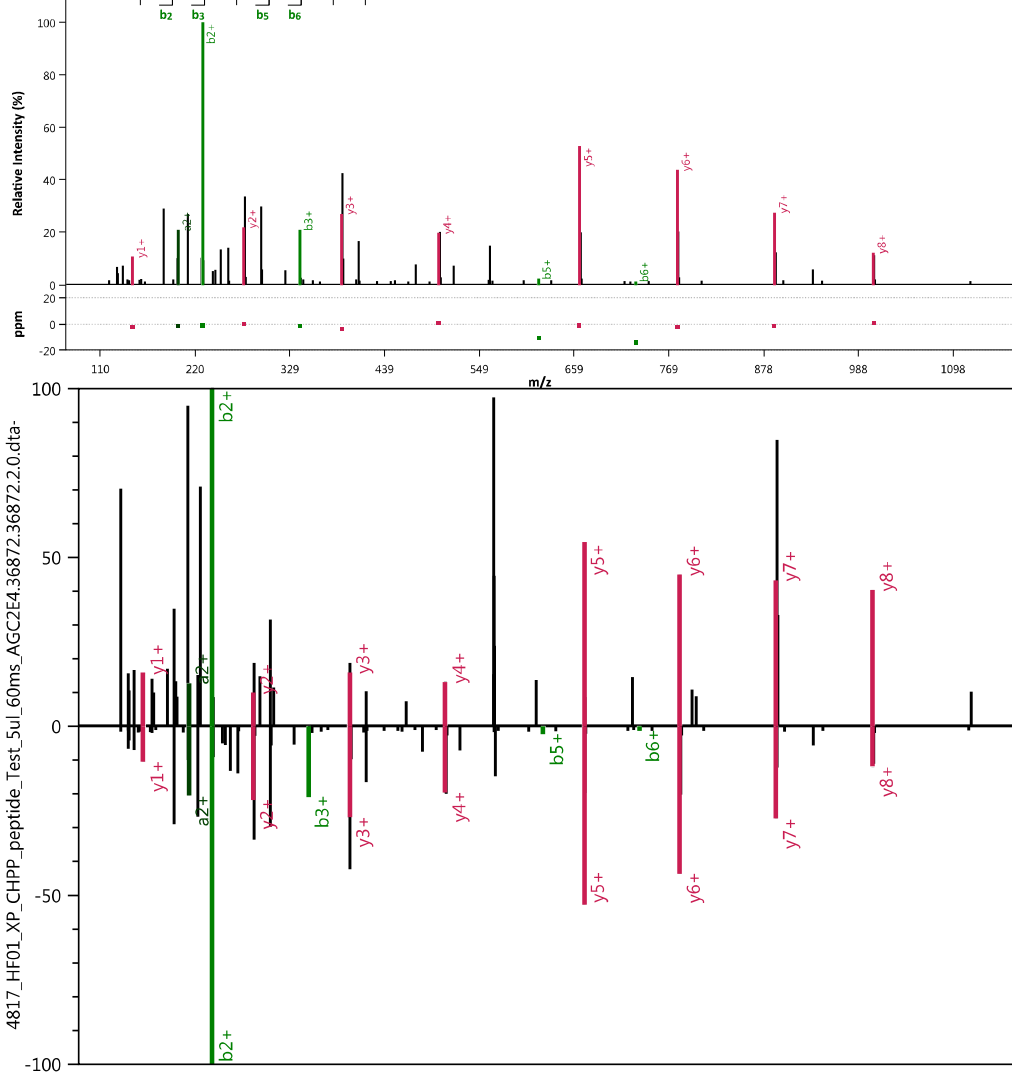
Title: 4817_HF01_XP_CHPP_Has_Tricine20_15_Sul_60ms_AGC2E4.32681.32681.2.0.dta Label: None Info: 4817_HF01_XP_CHPP_Has__Tricine20_15_Sul_60ms_AGC2E4 Base Peak: 9.12E+005 MS2_Mass: 1215.627377Da / 608.317327Th MS2_mass - Theoretical_Mass:0.001851Da/ 1.523ppm PSM_Score (\%): 17.342

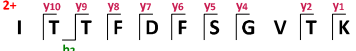

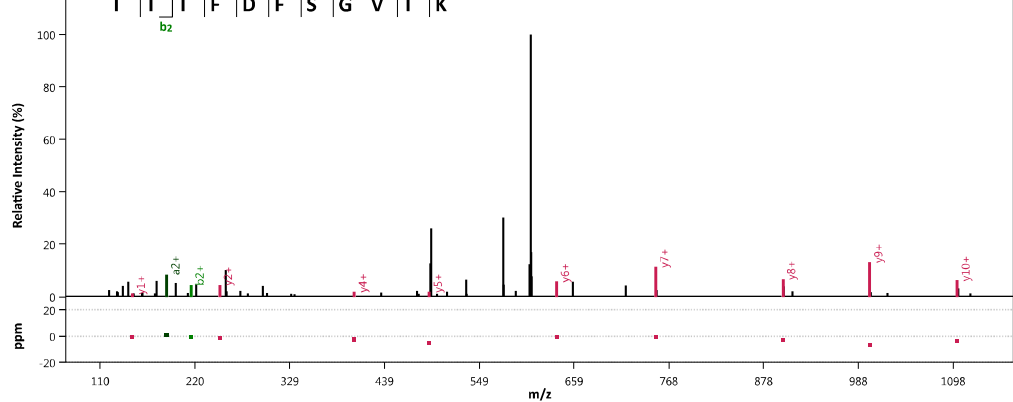

$\frac{1}{0}$
$\frac{1}{0}$
$\frac{0}{0}$
$\frac{2}{0}$
$\frac{d}{N}$
$\frac{N}{9}$
$\frac{1}{+}$
क
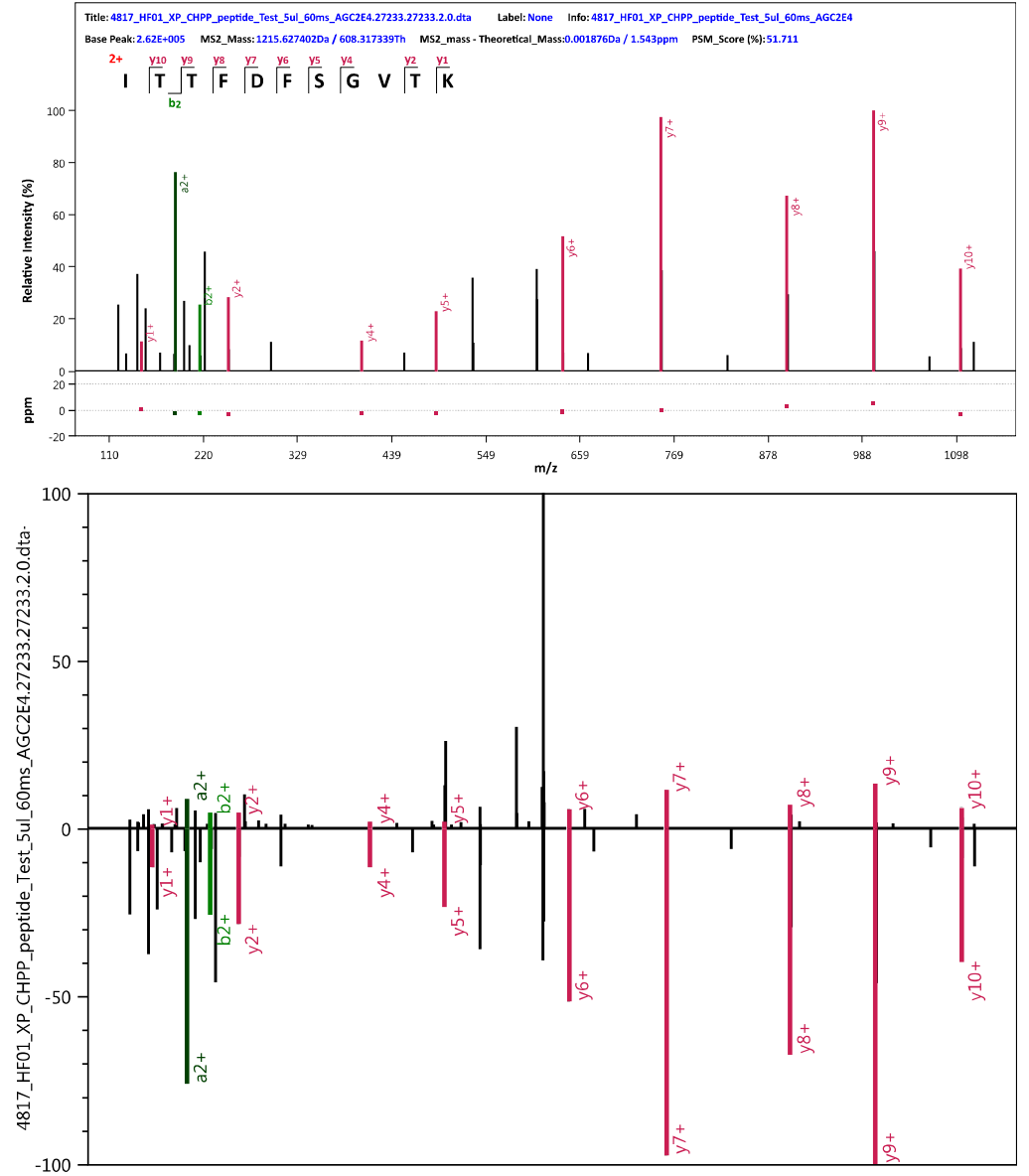


\section{SIHVIQDLVNEEPR}

$\operatorname{COS}=0.99$

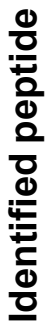

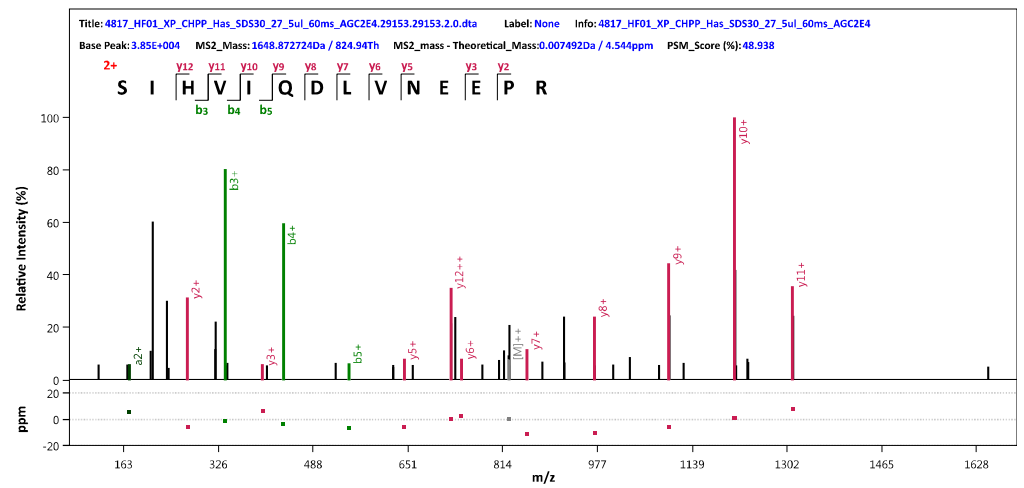

के
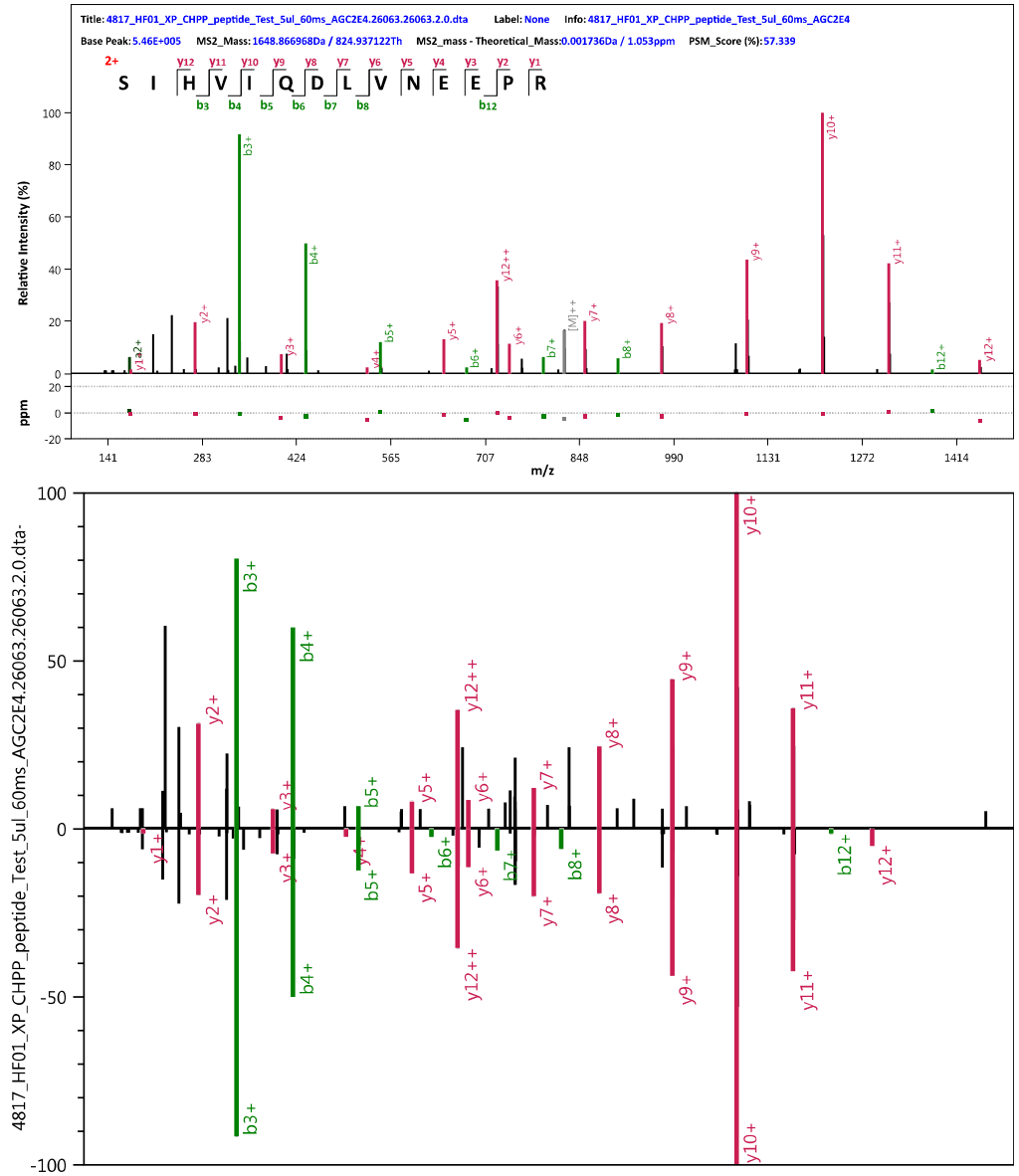


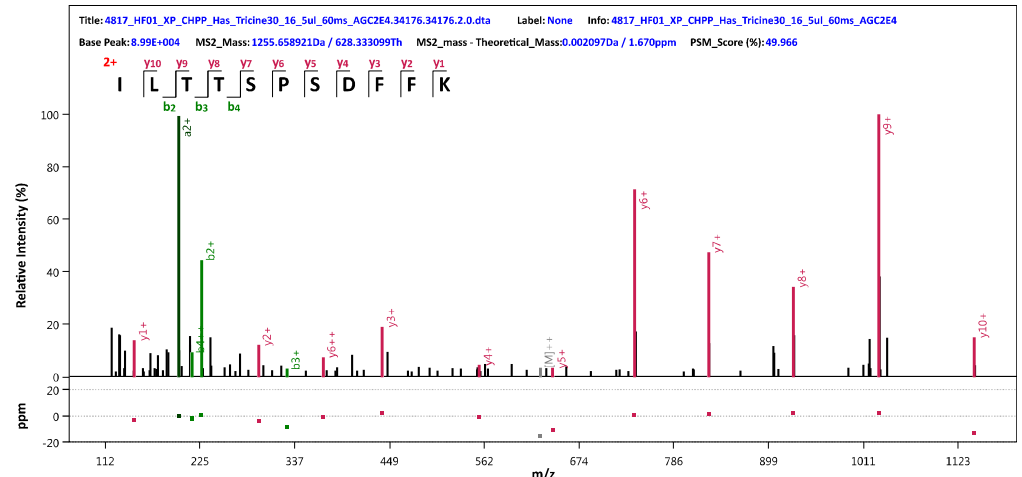

竞
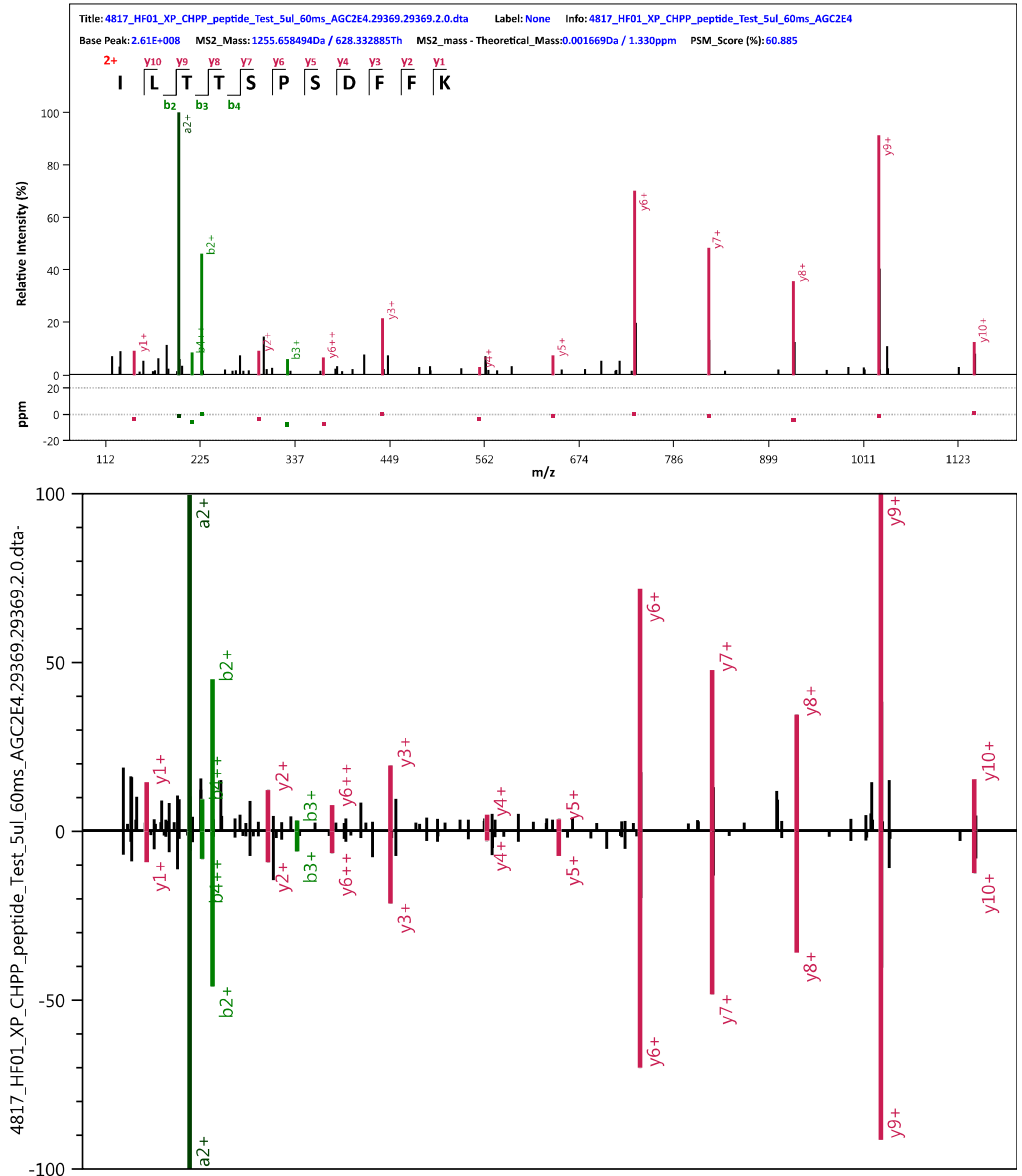
Title: 4817_HF01_XP_CHPP_Has_SDS10_27_6ul_60ms_AGC2E4.33176.33176.3.0.dta Label: None Info: 4817_HF01_XP_CHPP_Has_SDS10_27_6ul_60ms_AGC2E4

Base Peak: 1.13E+005 MS2_Mass:2729.245695Da / 910.420083Th MS2_mass - Theoretical_Mass:0.008565Da/3.138ppm PSM_Score (\%):36.534

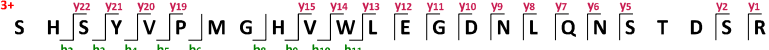

总

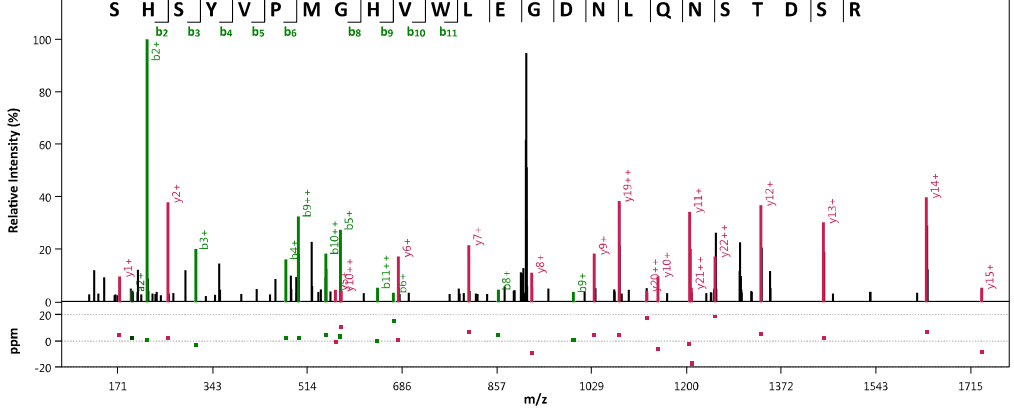

Title: 4817_HF01_XP_CHPP_peptide_Test_5ul_60ms_AGC2E4.25015.25015.3.0.dta Label: None Info: 4817_HF01_XP_CHPP_peptide_Test__5ul_60ms_AGC2E4

Base Peak:2.52E+007 MS2_Mass: 2729.237209Da / 910.417254Th MS2_mass - Theoretical_Mass:0.000079Da / 0.029ppm PSM_Score (\%): 52.245

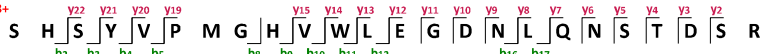
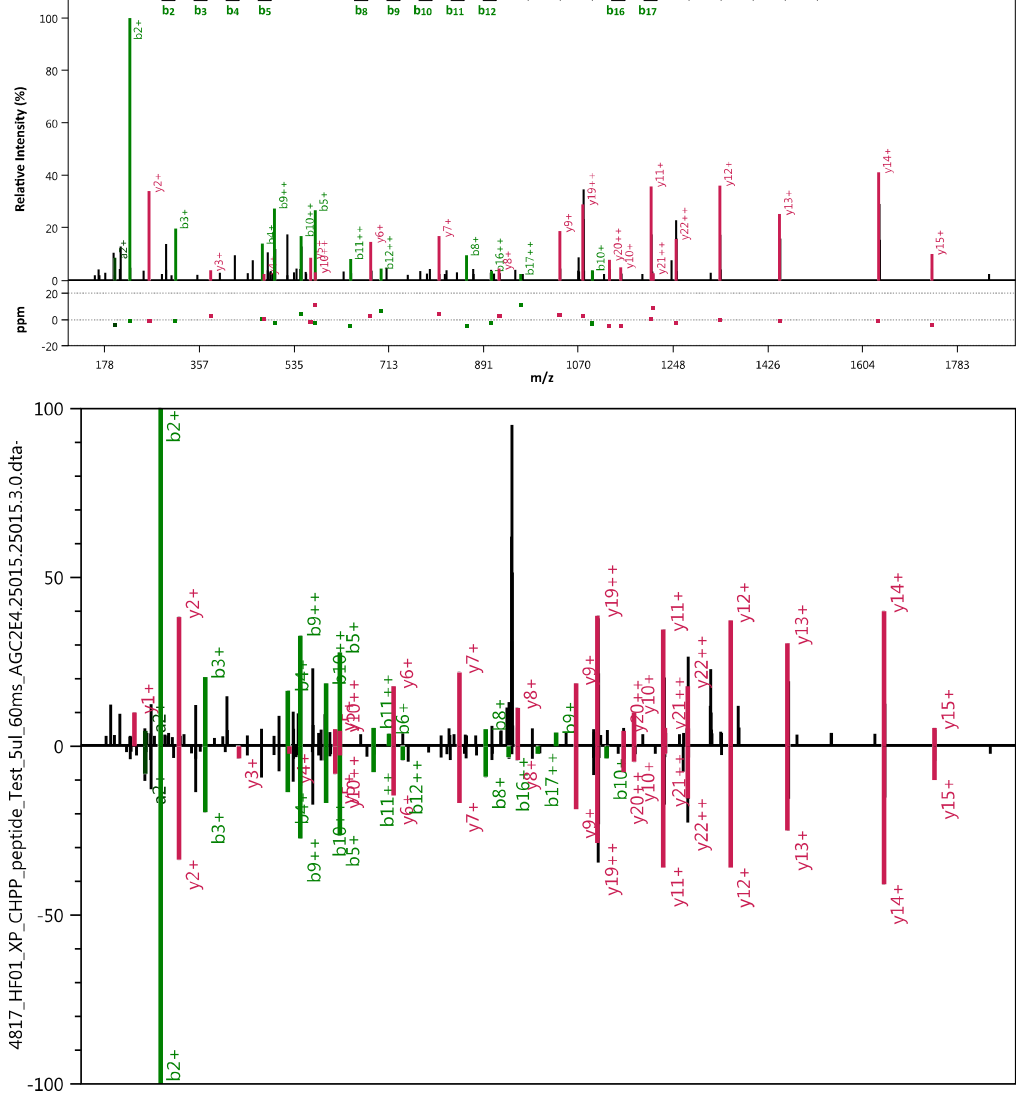
Title: 4817_HF01_XP_CHPP_Has_SDS10_13_5ul_60ms_AGC2E4.2970.2970.2.0.dta Label: None Info: 4817_HF01_XP_CHPP_Has_SDS10_13_5ul_60ms_AGC2E4 Base Peak: 3.70E+004 MS2_Mass: 1123.515162Da / 562.261219Th MS2_mass - Theoretical_Mass:0.002537Da/ 2.258ppm PSM_Score (\%): 58.164

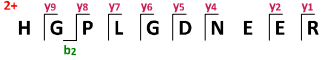

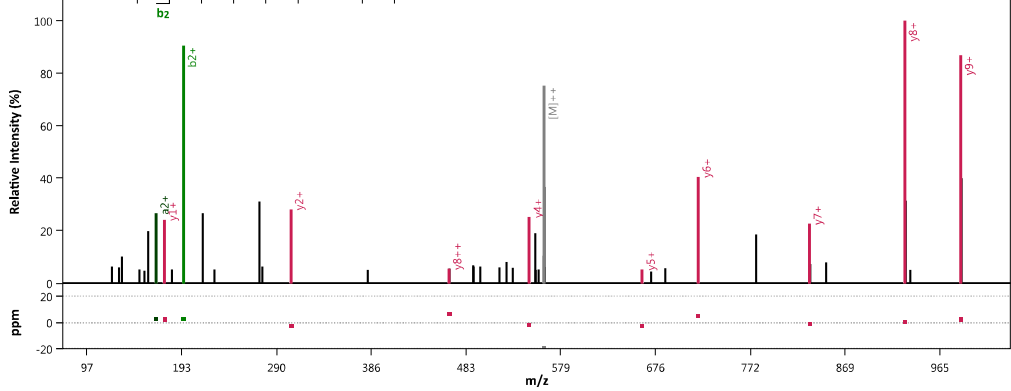

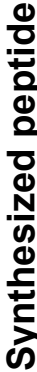
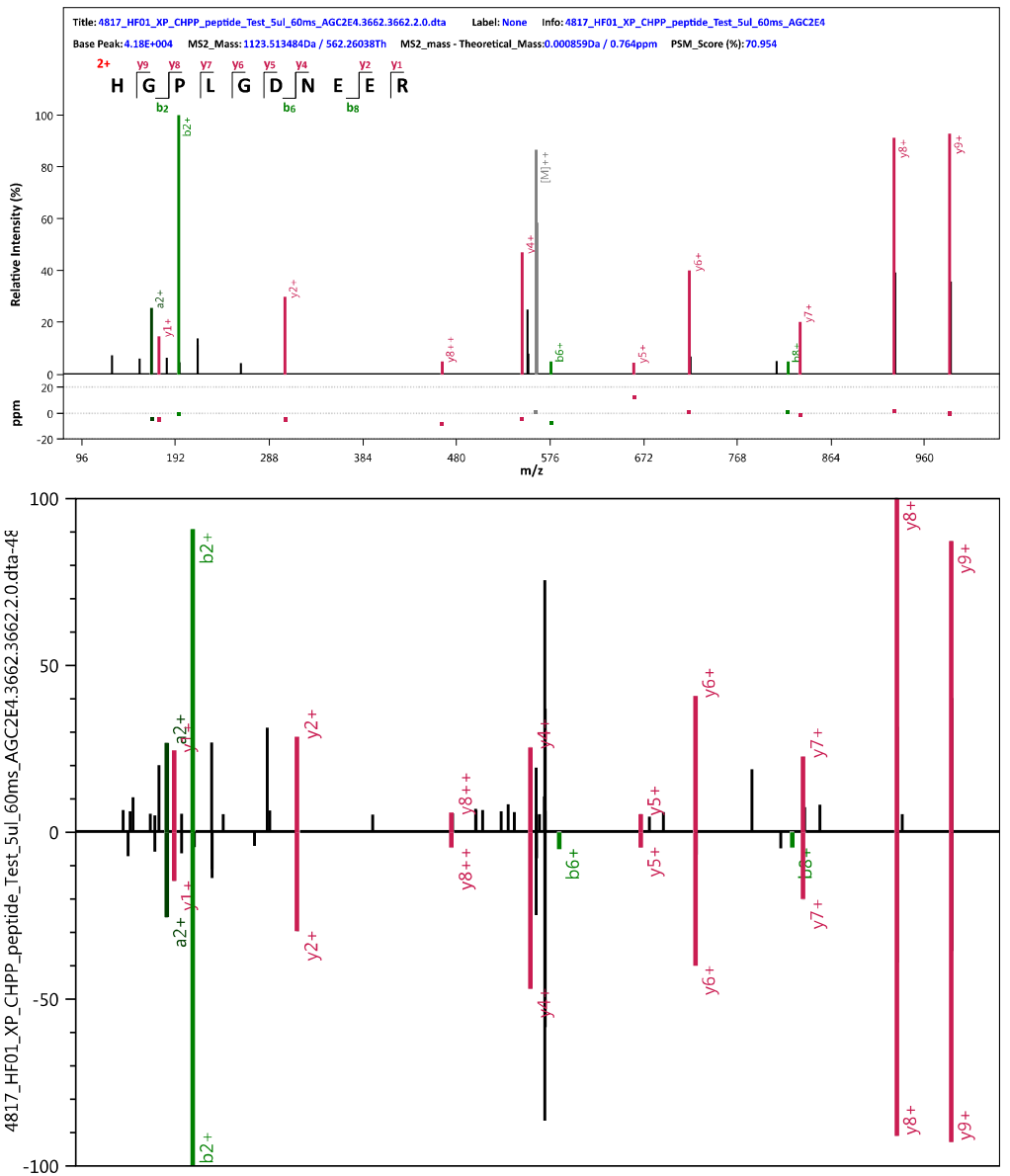
Title: 4817_HF01_XP_CHPP_Has_SDS10_13_5ul_60ms_AGC2E4.19559.19559.2.0.dta Label:None Info: 4817_HF01_XP_CHPP_Has_SDS10_13_5ul_60ms_AGC2E4 Base Peak: 5.16E+004 MS2_Mass: 15499.772724Da / 775.39Th MS2_mass - Theoretical_Mass:0.008916Da / 5.753ppm PSM_Score (\%): 55.902

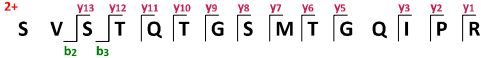

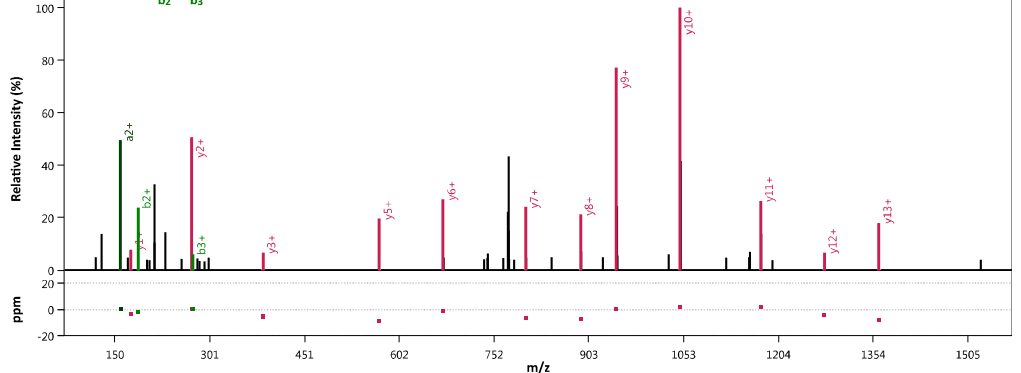

क्षे

Title: 4817_HF01_XP_CHPP_peptide_Test_5ul_60ms_AGC2E4.15465.15465.2.0.dta Label: None Info: 4817_HF01_XP_CHPP_peptide_Test_5U1_60ms_AGC2F4

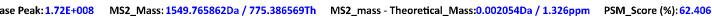

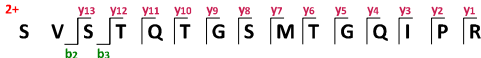
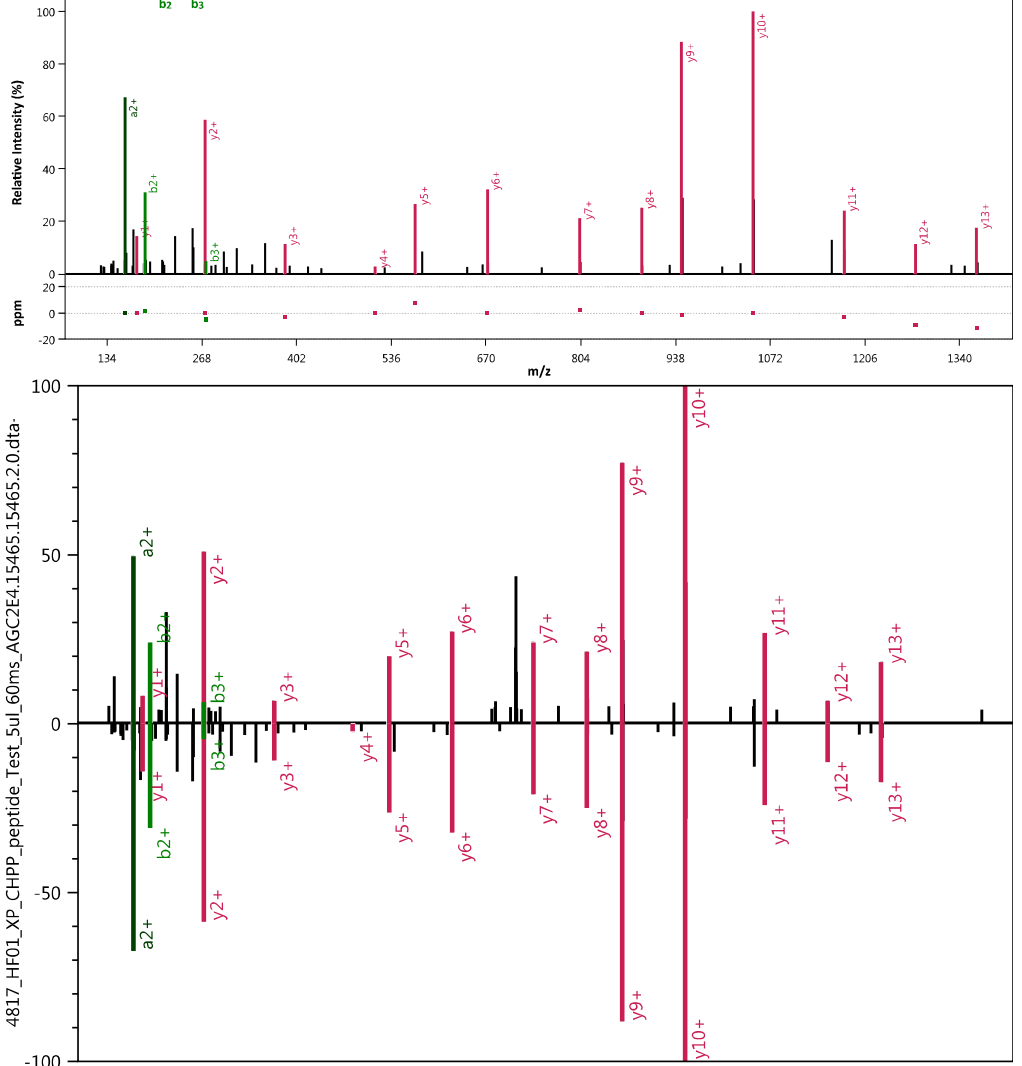
TLPDANTDFYYECK

$\cos =0.93$
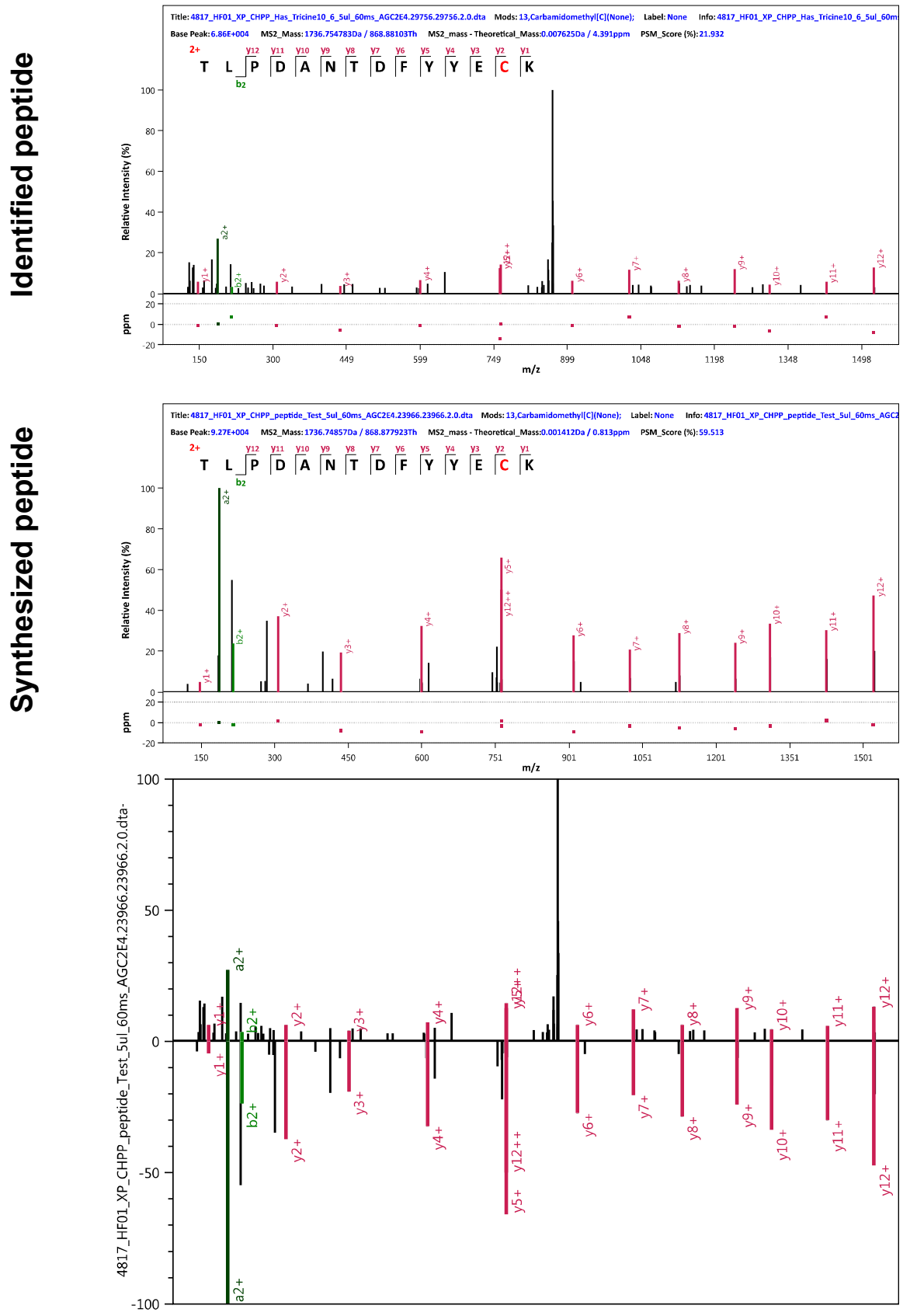

S-75 


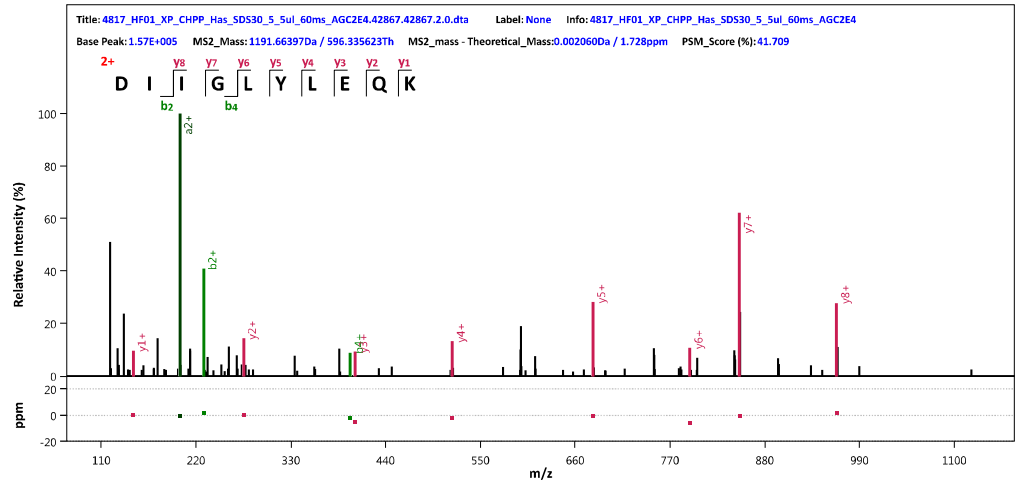

ํㅡㄹ
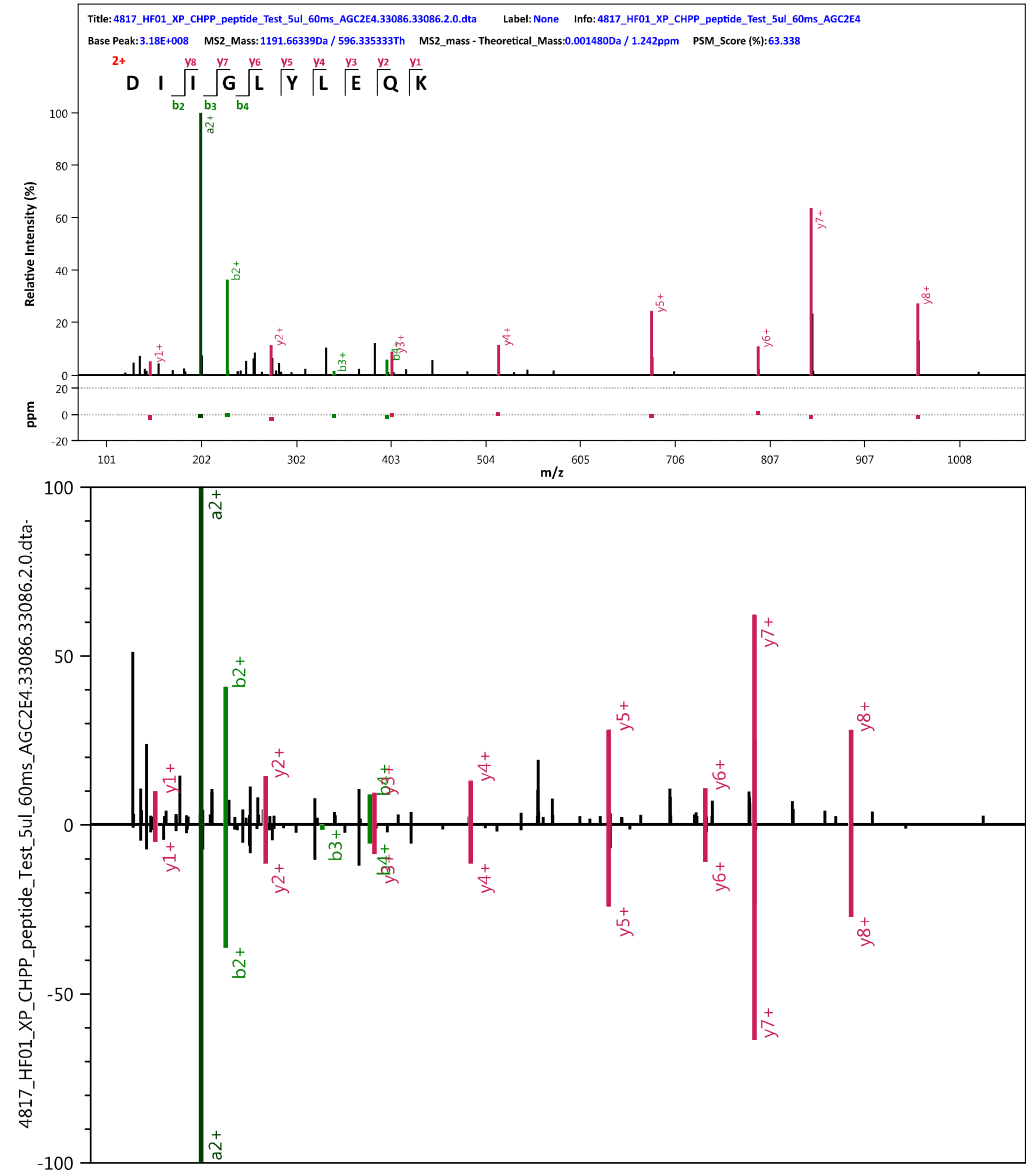


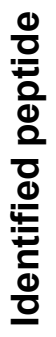

Title: 4817_HF01_XP_CHPP_Has_SDS30_5_5ul_60ms_AGC2E4.15056.15056.2.0.dta Label: None Info:4817_HF01_XP_CHPP_Has_SDS30_5_5ul_60ms_AGC2EA

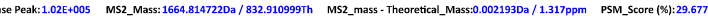

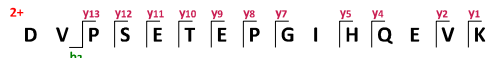

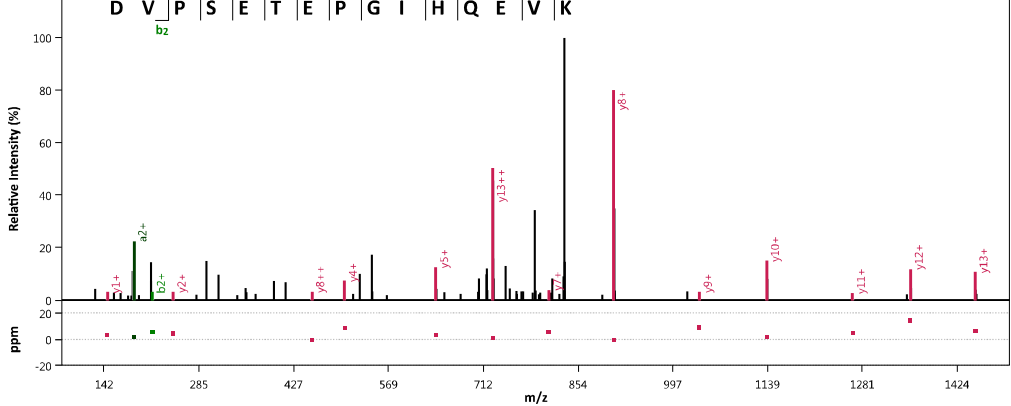

을

Title: 4817_HF01_XP_CHPP_peptide_Test_5ul_60ms_AGC2E4.11540.11540.2.0.dta Label: None Info: 4817_HF01_XP_CHPP_Peptide_Test_5ul_60ms_AGC2E4

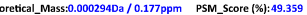

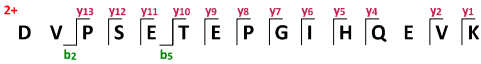
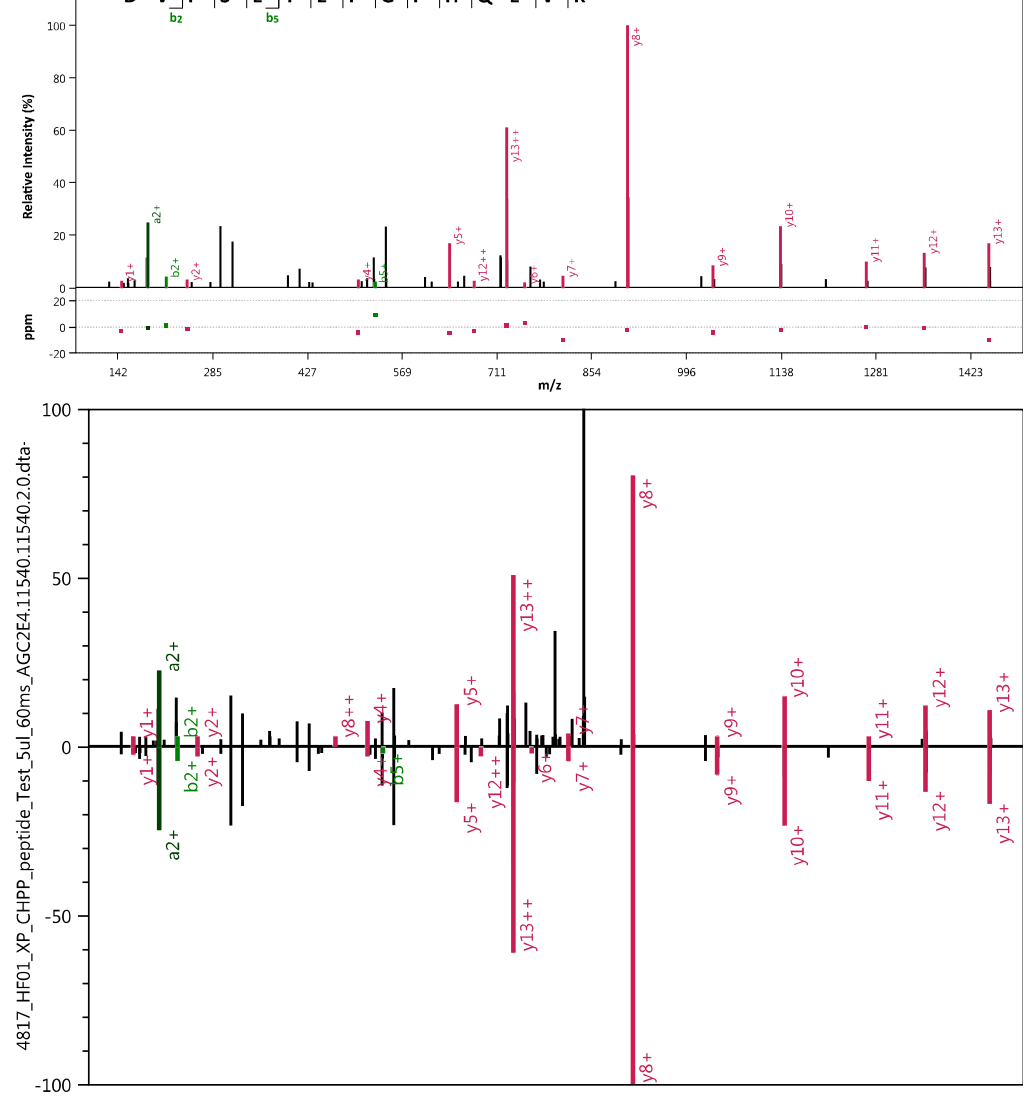


\section{NLELLEEETEPGVPEESLR}

Dase Peak:2.78E+005 MS2_Mass:2183.075367Da / 1092.041322Th MS2_mass - Theoretical Mass.0.000069Da/1.864ppm PSM_Score (6):49.54

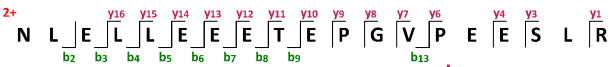

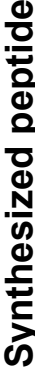
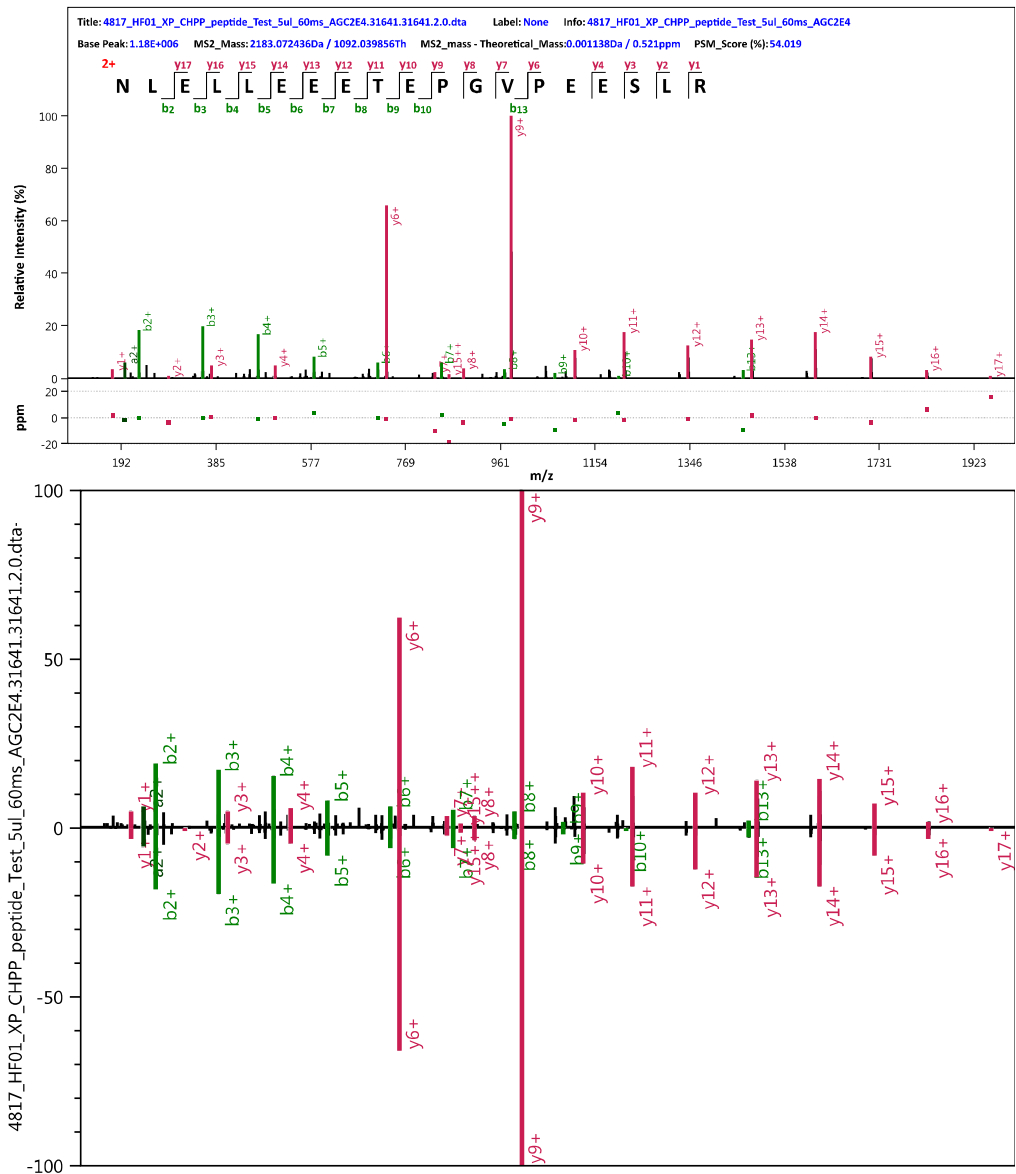

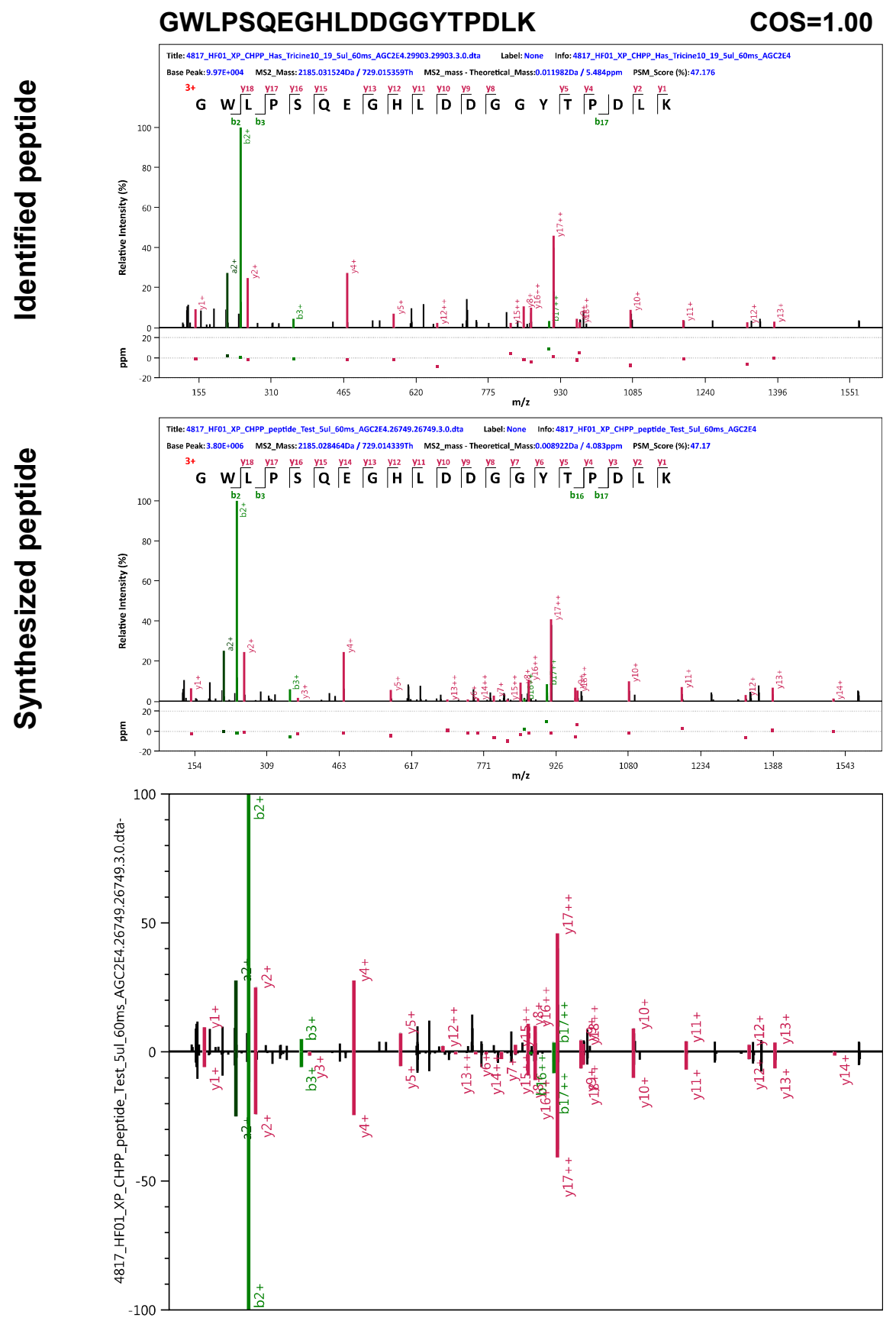

S-79 


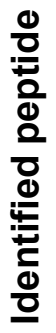

Title: 4817_HF01_XP_CHPP_Has_SOS10_10_5ul_60ms_AGC2E4.40914.40914.2.0.dta Label: None Info: 4817_HF01_XP_CHPP_Has_SDS10_10_5ul_60ms_AGC2E4 Base Peak: 1.33E+005 MS2_Mass: 1544.775914Da / 772.891595Th MS2_mass - Theoretical_Mass:0.001612Da/1.044ppm PSM_Score (\%):44.913

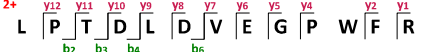

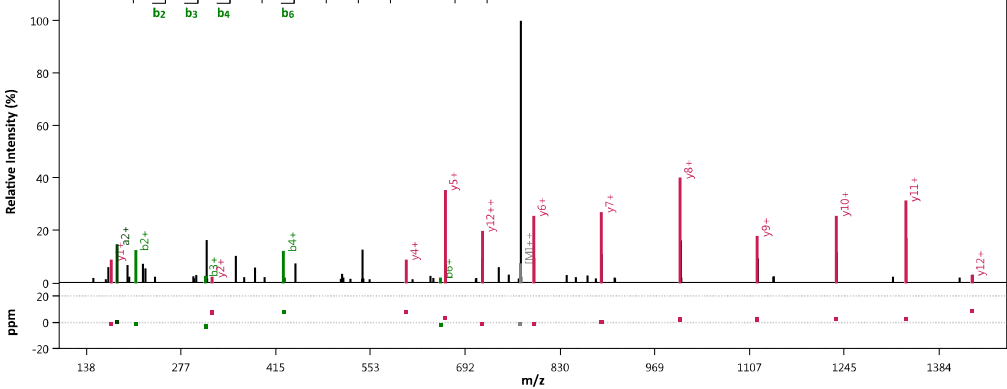

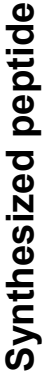
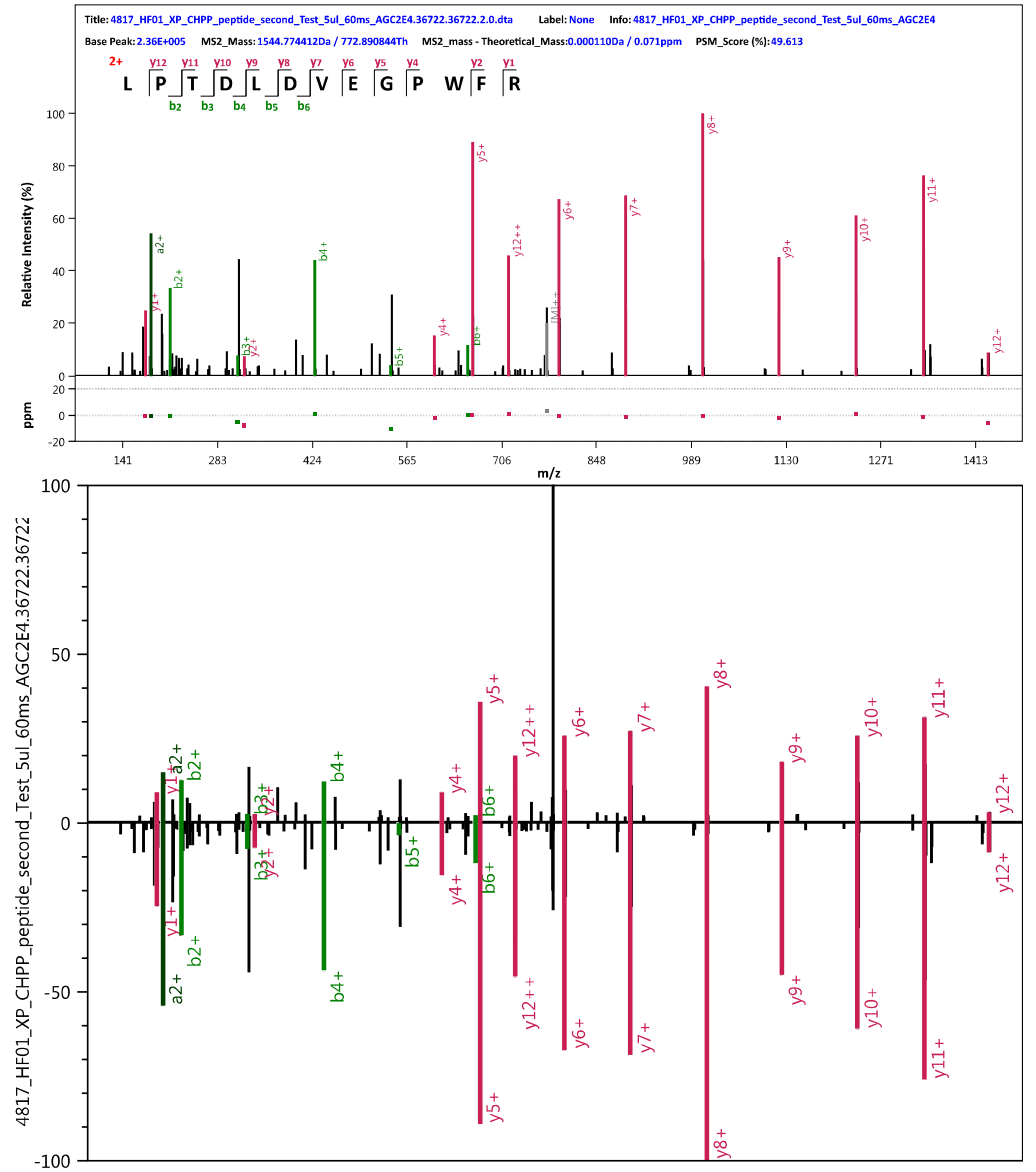
Title: 4817_HF01_XP_CHPP_Has_Tridne10_5_6ul_60ms_AGC2E4.28483.28483.2.1.dta Label: None Info: 4817_HF01_XP_CHPP_Has_Trikine10_5_6ul_60ms_AGC2E4 5.40E+005 MS2_Mass: 1754.801144Da / 877.90421Th MS2 mass -Theoretical_Mass:0.003203Da/ 1.825ppm PSM_Score (\%):29.243

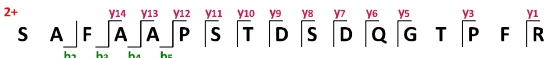

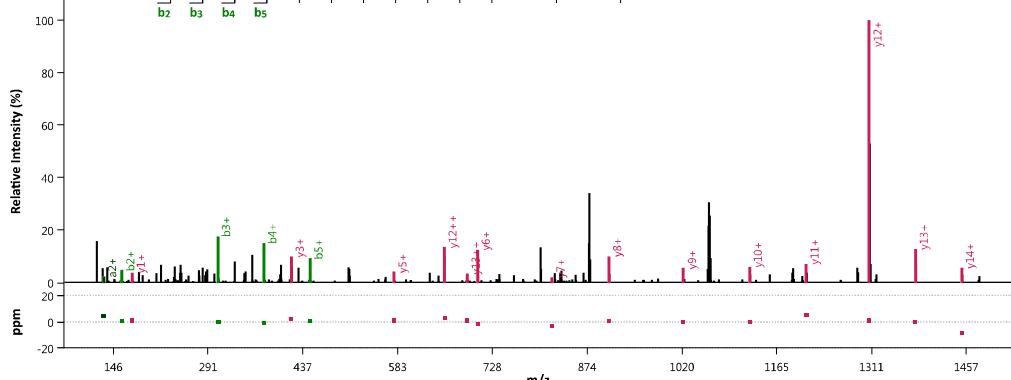

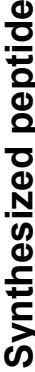

Title: 4817_HF01_XP_CHPP_peptide_second_Test_5ul_60ms_AGC2E4.20247.20247.2.0.dta Label: None Info: 4817_HF01_XP_CHPP_peptide_second_Test_5ul_60ms_AGC2E4 Base Peak: 5.44E+006 MS2_Mass: 1754.799848Da / 877.903562Th MS2_mass - Theoretical_Mass:0.001907Da / 1.087ppm PSM_Score (\$):51.271

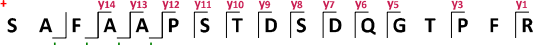
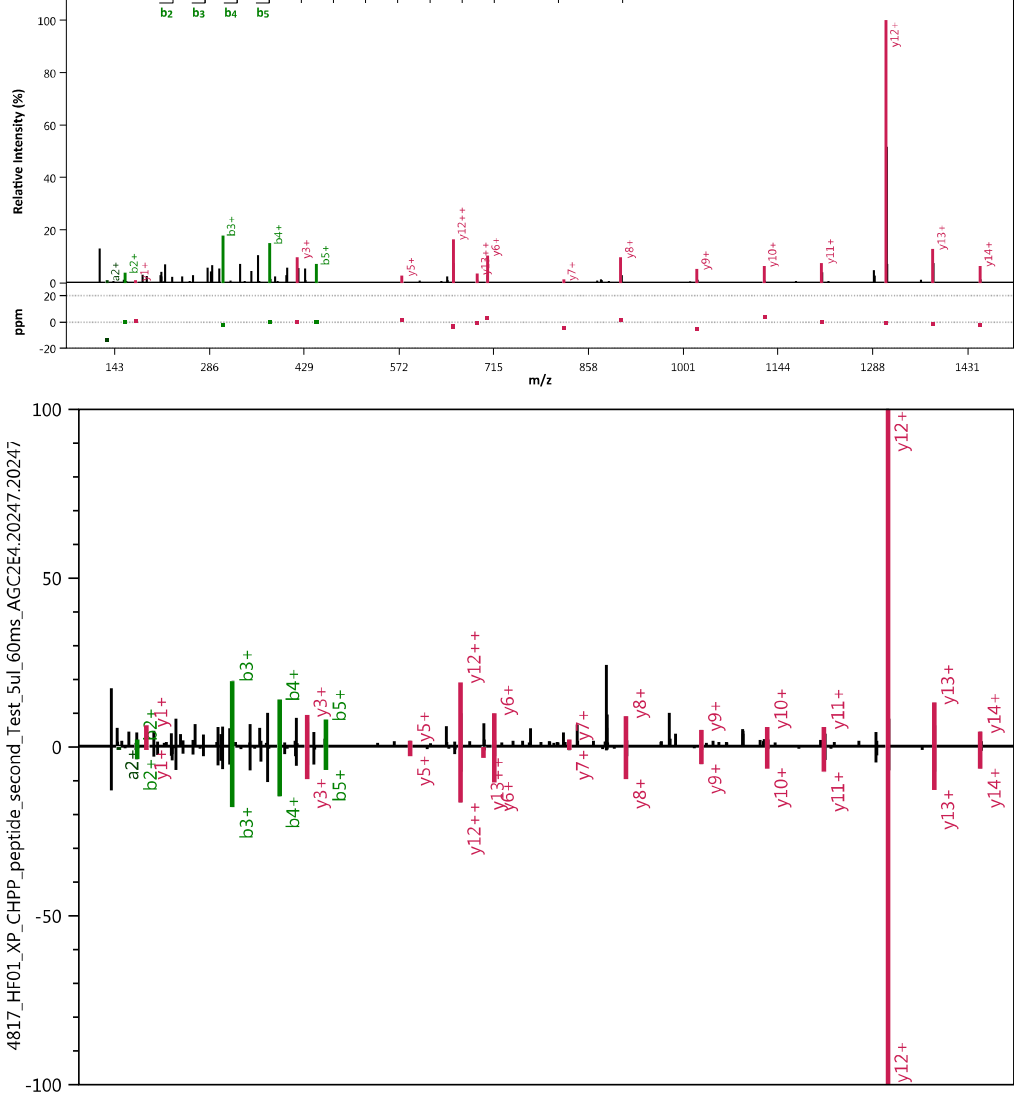
作 Base Peak:1.00E+004 MS2_Mass: 1777.793226Da/889.400251Th MS2_mass - Theoretical_Mass:0.002420Da/1.361ppm PSM_Score (\%): 53.652

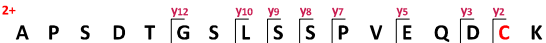

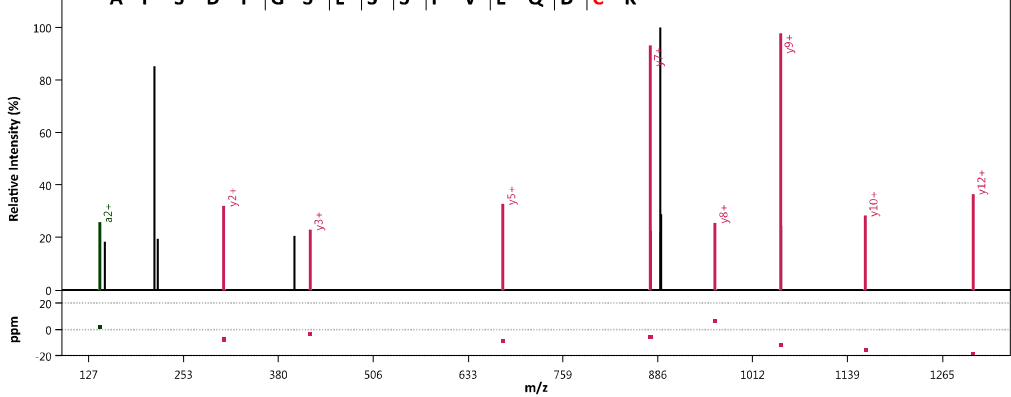

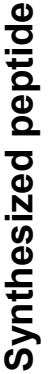
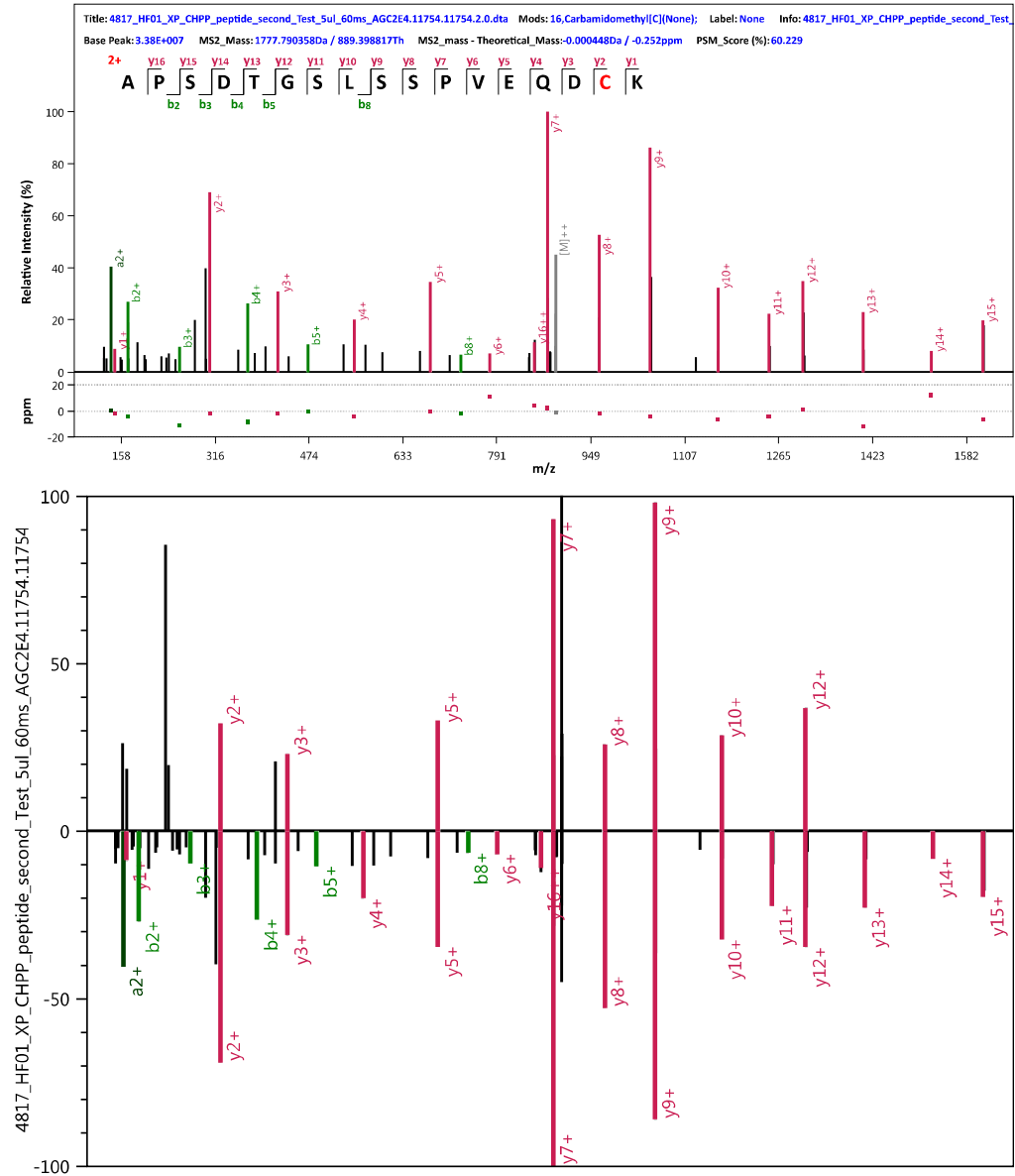
Title: 4817_HF01_XP_CHPP_Has__DDS10_5_5ul_60ms_AGC2E4.9264.9264.2.0.dta Label: None Info: 4817_HF01_XP_CHPP_Has_SDS10_5_5ul_60mi__GGC2E4 Base Peak: 1.02E+005 MS2_Mass: 1584.76545Da / 792.886363Th MS2_mass- Theoretical_Mass:0.004285Da / 2.704ppm PSM_Score (x): 38.89

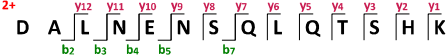

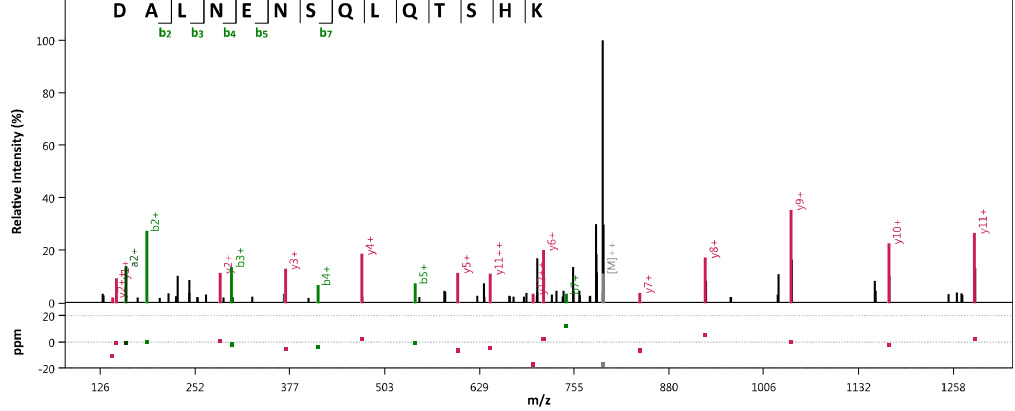

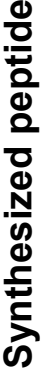

Title: 4817_HF01_XP_CHPP_peptide_second_Test_Sul_60ms_AGC2E4.6666.6666.20.dta Label:NNone Info:4817_HF01_XP_CHPP_peptide_second_Test_5ul_60ms_AGC2E4 Base Peak: 4.11E+004 MS2_Mass: 1584.760342Da / 792.883809Th MS2_mass - Theoretical_Mass:-0.000823Da / - 0.519 ppm PSM_Score (\%): 55.091

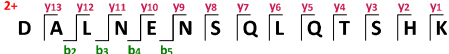
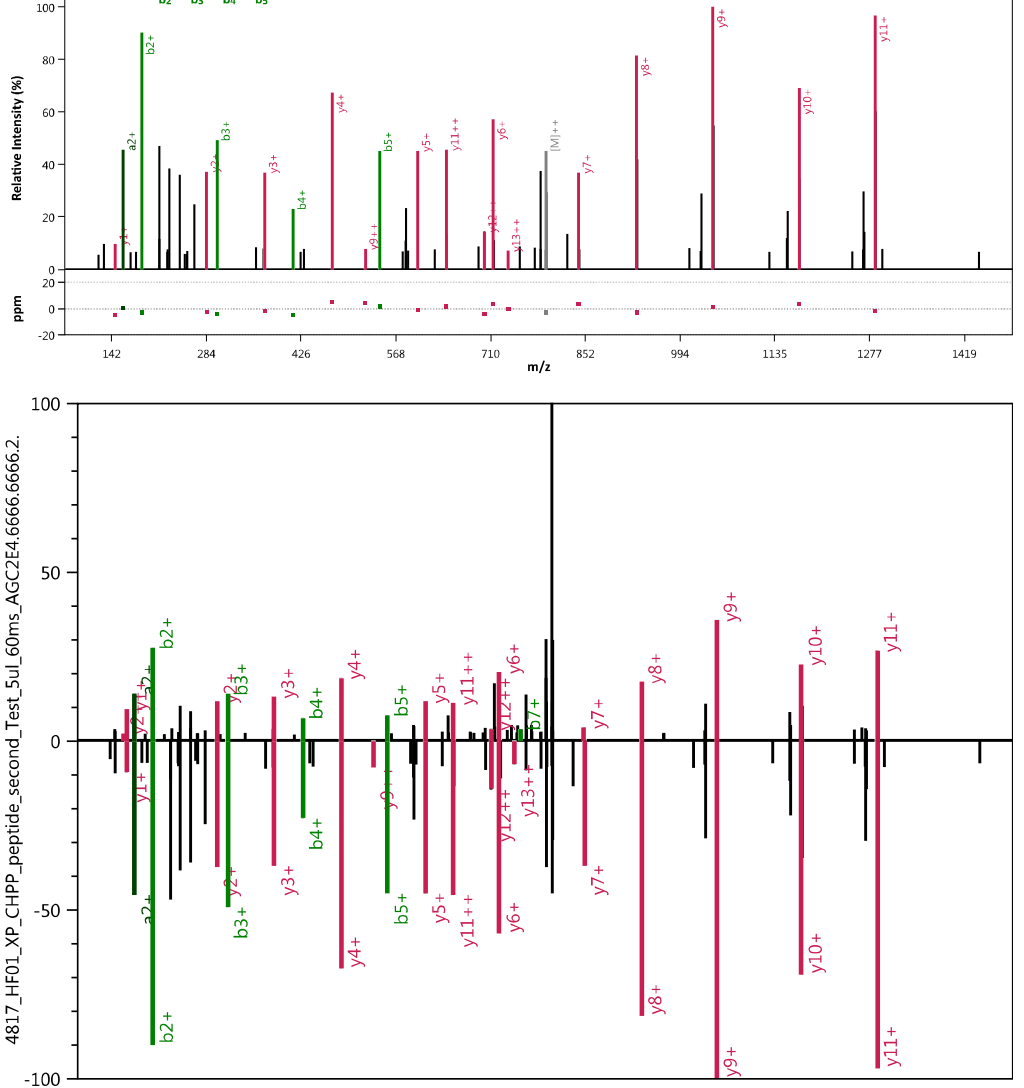
Title: 4817_HF01_XP_CHPP_Has_SDS10_5_5ul_60ms_AGC2E4.16293.16293.2.0.dta Label: None Info: 4817_HF01_XP_CHPP_Has_SDS10_5_5ul_60ms_AGC2EA

Base Peak: 3.24E+005 MS2_Mass: 1135.551635Da / 568.279456Th MS2_mass - Theoretical_Mass:0.003964Da/3.491ppm PSM_Score (\%): 67.466

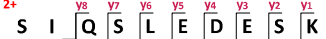

送
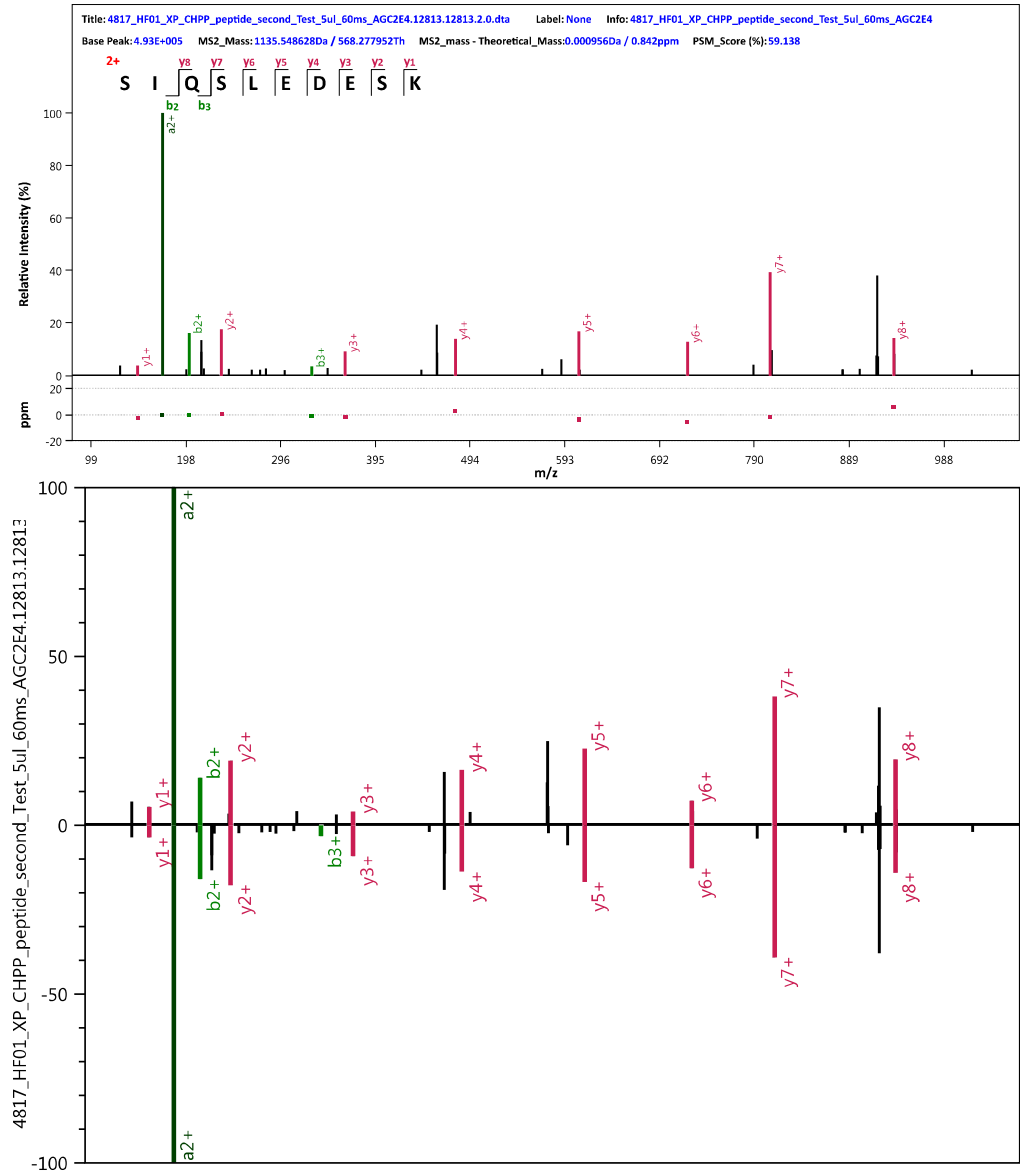


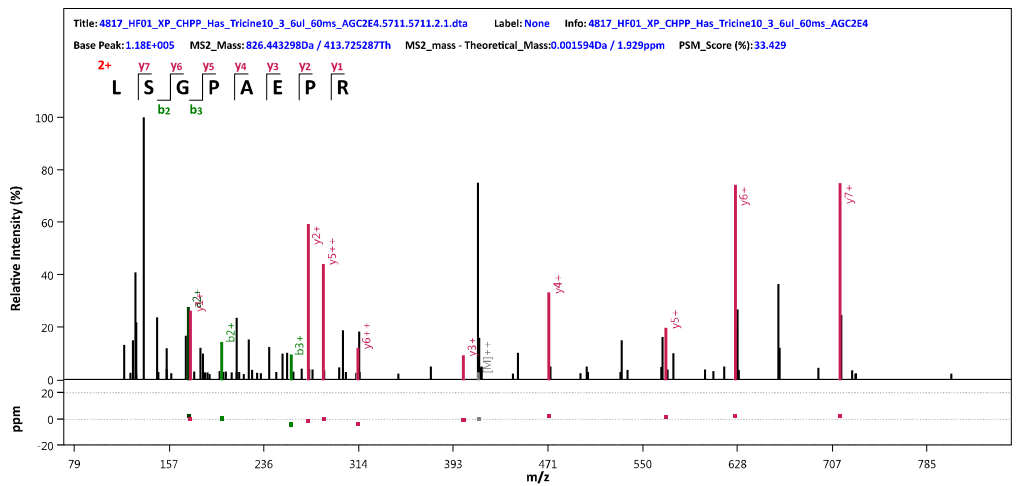

告
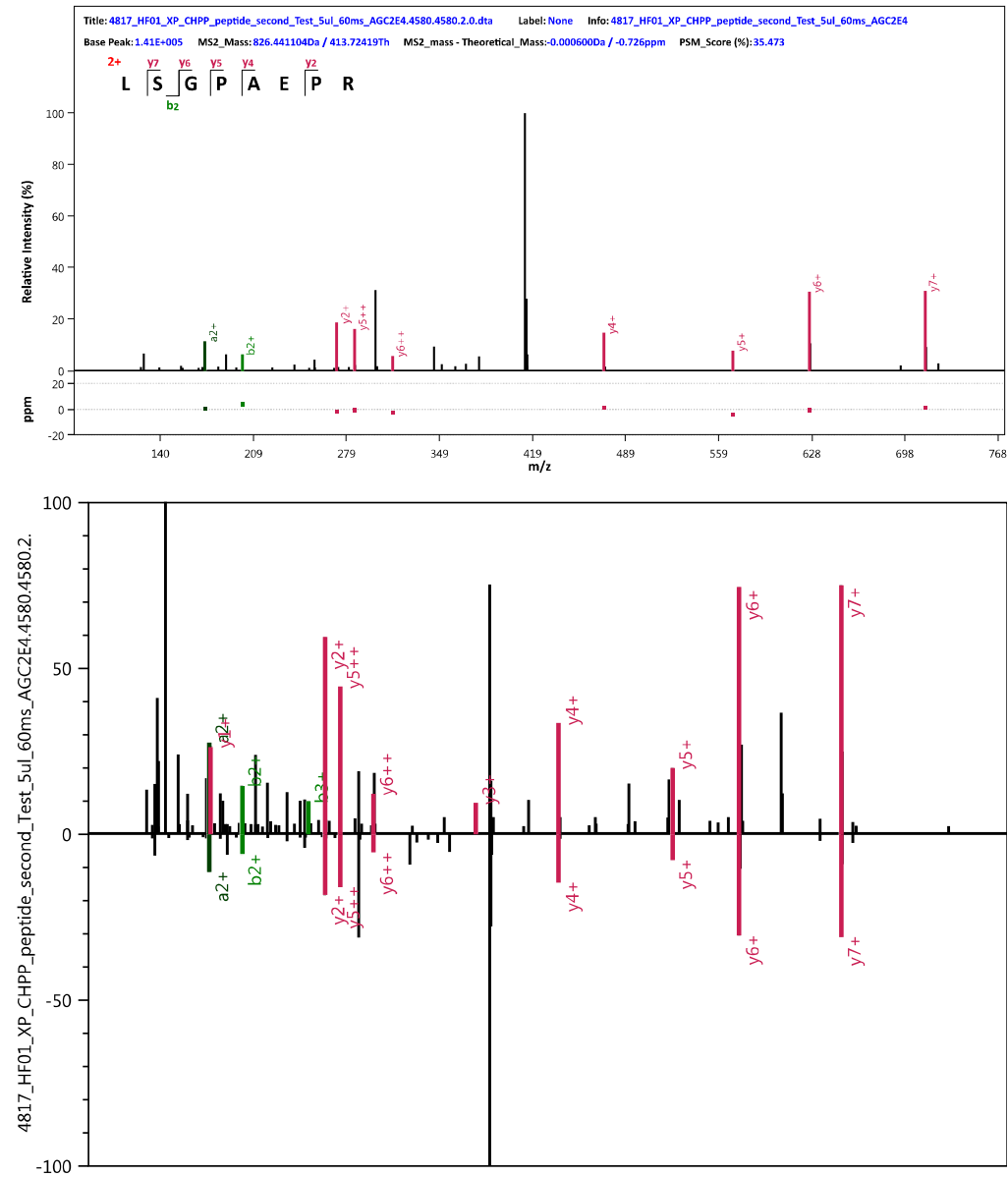
Title: 4817_HF01_XP_CHPP_Has_SDS20_24_6ul_60ms_AGC2E4.14945.14945.2.0.dta Label: None Info: 4817_HF01_XP_CHPP_Has_SDS20_24_6ul_60ms_AGC2E4 Base Peak:9.65E+004 MS2_Mass: 1332.642454Da / 666.824865Th MS2_mass - Theoretical_Mass:0.003518Da/2.640ppm PSM_Score (\%): 55.703

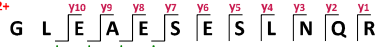

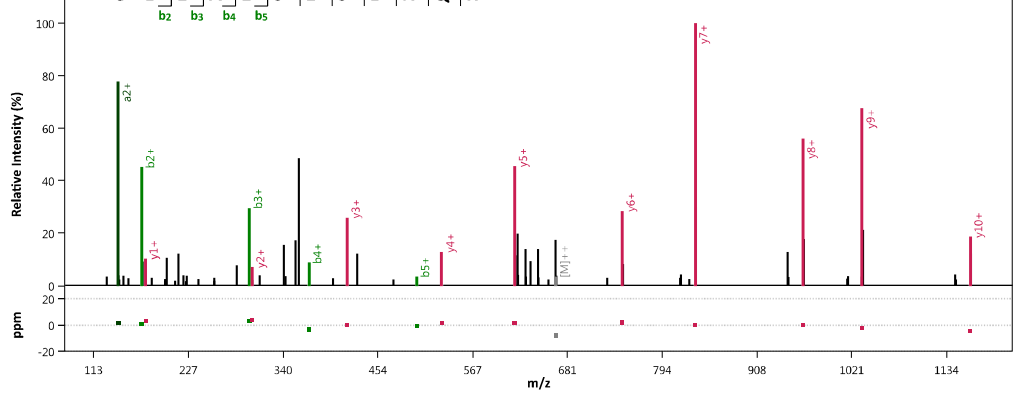

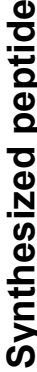

Title: 4817_HF01_XP_CHPP_peptide_second_Test_5ul_60ms_AGC2E4.11918.11918.2.0.dta Label: None Info: 4817_HF01_XP_CHPP_peptide_second_Test_5ul_60ms_AGC2E4 Base Peak: 1.94E+005 MS2_Mass: 1332.639992Da / 666.823634Th MS2_mass - Theoretical_Mass:0.001056Da / 0.792 ppm PSM_Score (\%): 52.647

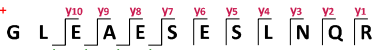
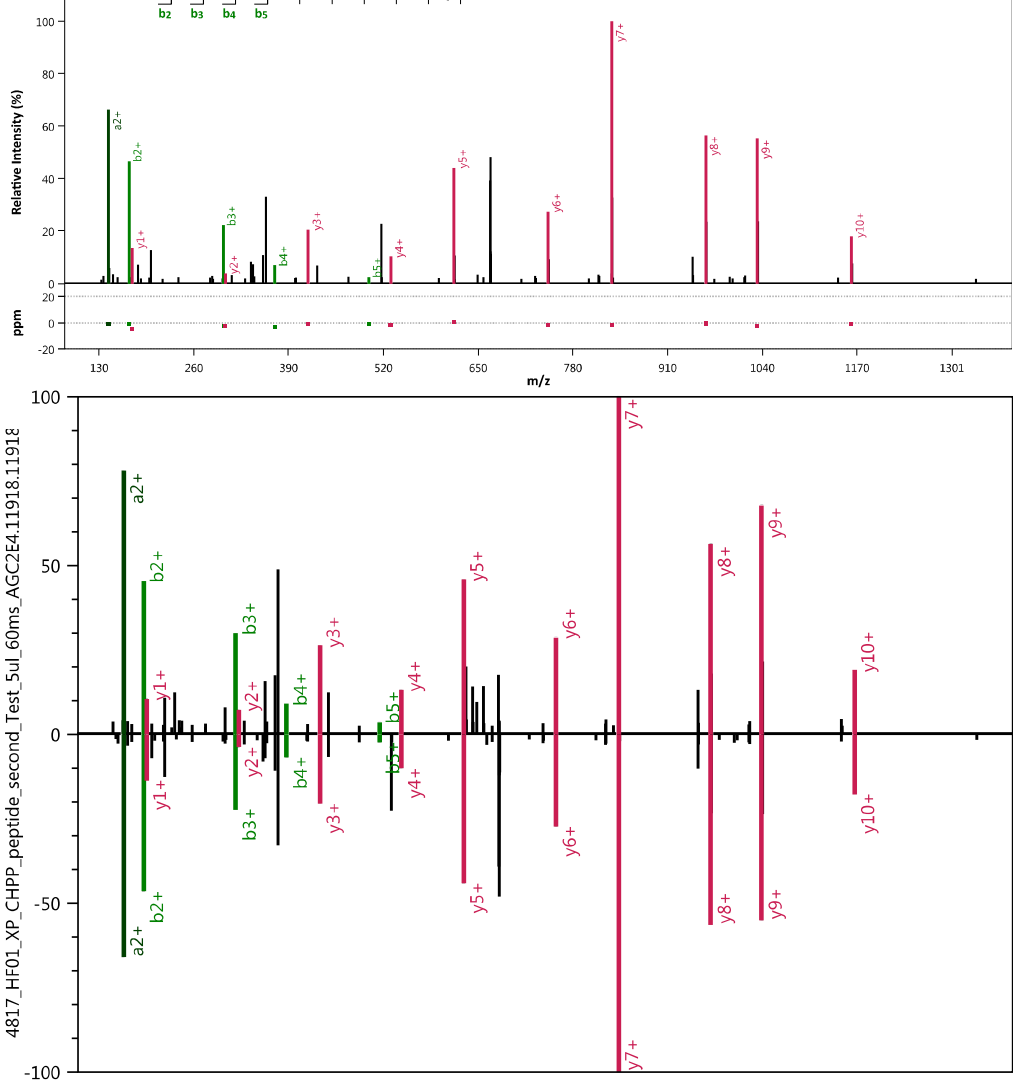


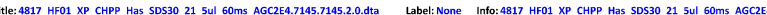
Base Peak:1.11E+005 MS2_Mass: 1255.639665Da / 628.323471Th MS2_mass - Theoretical_Mass:0.000787Da / 0.626ppm PSM_Score (\%):51.209

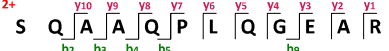

을

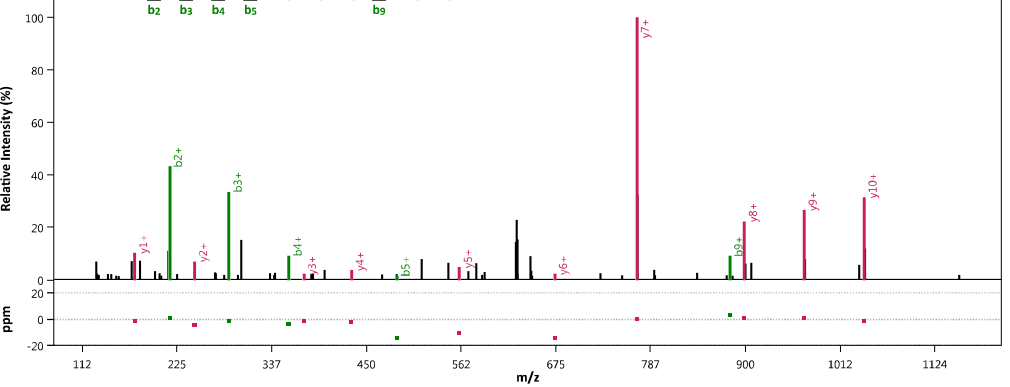

Title: 4817_HF01_XP_CHPP_peptide_Second_Test_5ul_60ms_AGC2E4.5169.5169.2.0.dta Label: None Info: 4817_HF01_XP_CHPP_peptide_second_Test_5ul_60ms_AGC2E4 Base Peak:1.58E+007 MS2_Mass:1255.638596Da / 628.322936Th MS2_mass - Theoretical_Mass:-0.000283Da / -0.226ppm PSM_Score (\%): 68.202

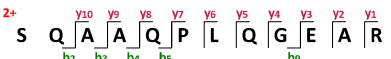
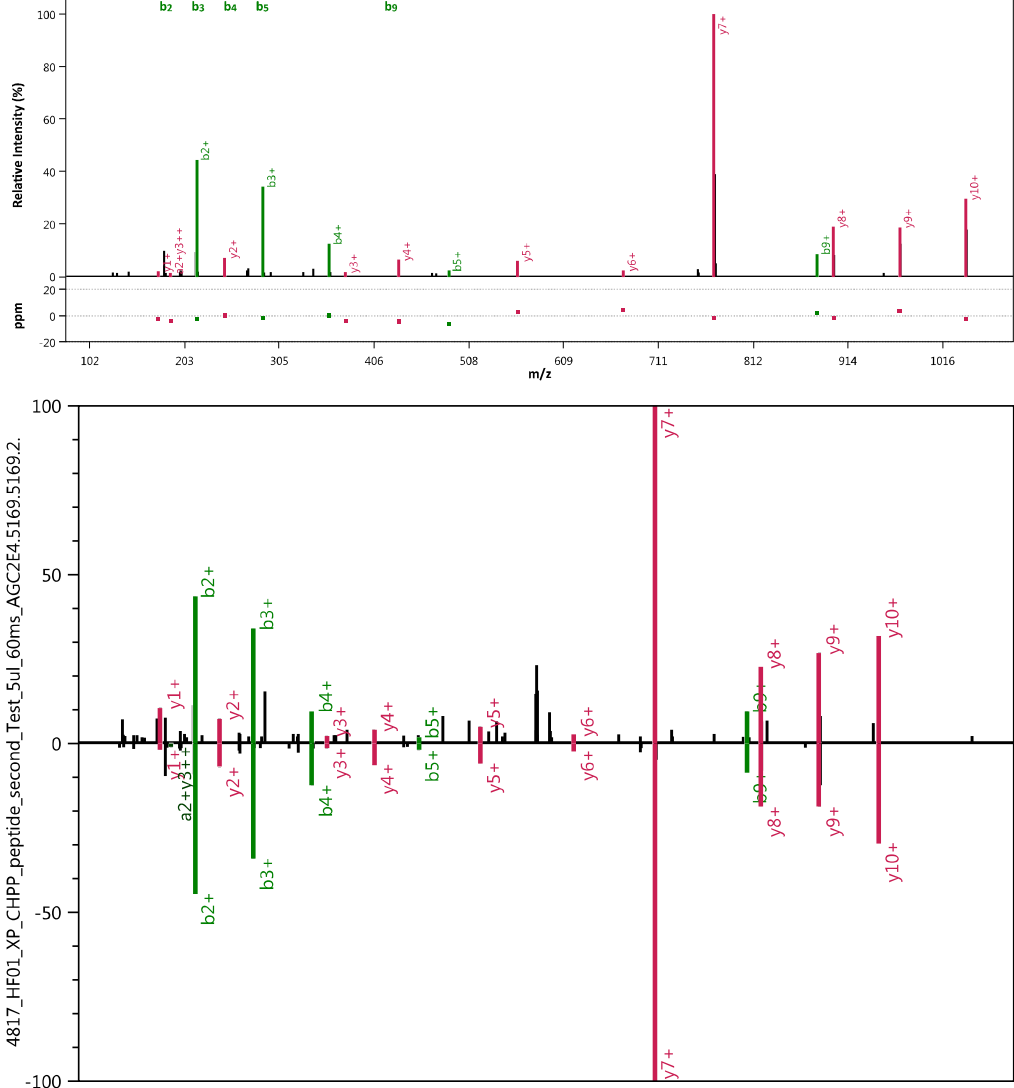


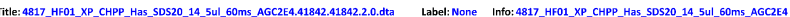
Base Peak: 1.81E+004 MS2 Mass: 1825.8770a/913.442138Th MS2 mass - Theoretical_Mass:0.0015390a/0.843ppm PSM Score (\%):67.359

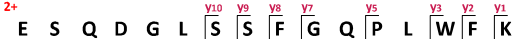

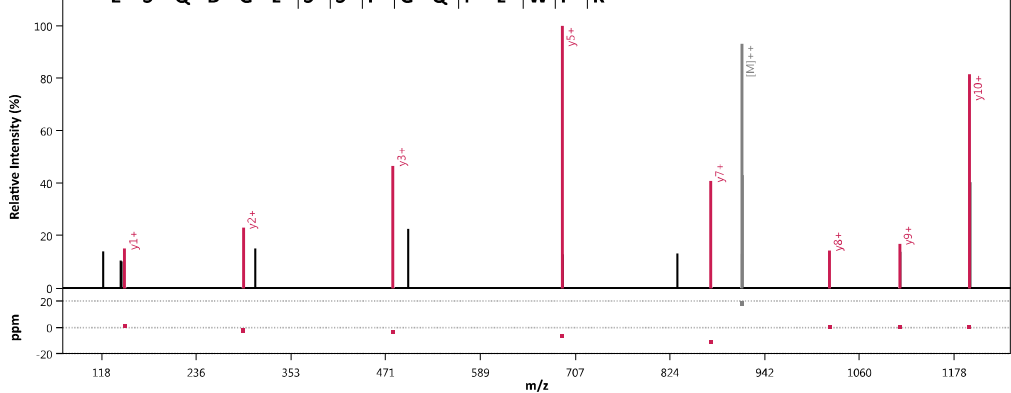

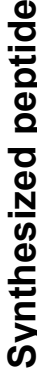
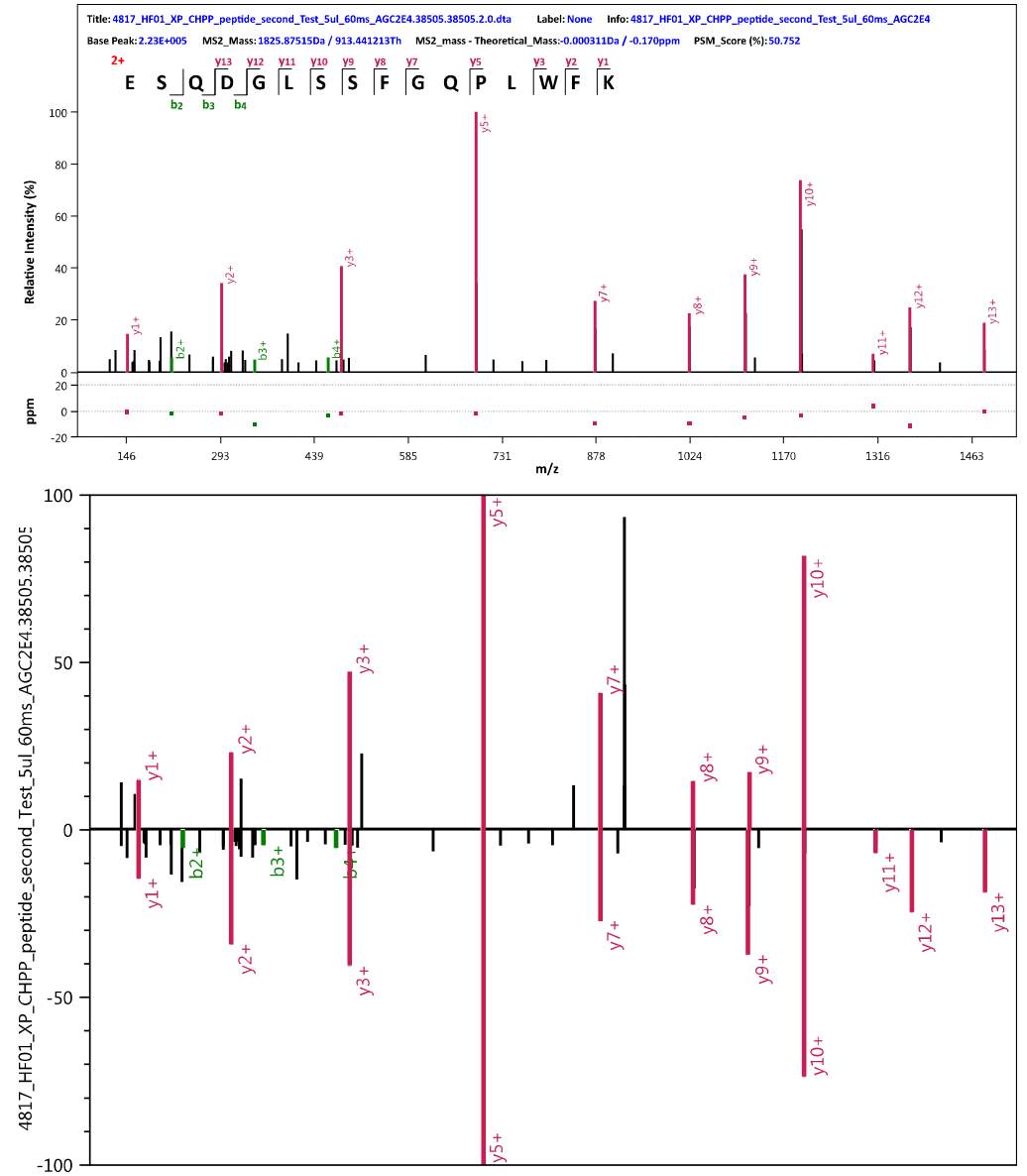
Titte: 4817_HF01_XP_CHPP_Has_SDS30_1_5ul_60ms_AGC2E4.49891.49891.2.0.dta Label: None Info: 4817_HF01_XP_CHPP_Has__SDS30_1_5ul_60ms_AGC2E4 Base Peak:2.02E+004 MS2_Mass: 1258.718782Da/629.863029Th MS2_mass - Theoretical_Mass:0.003443Da / 2.735ppm PSM_Score (\%):37.356

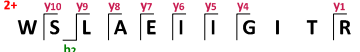

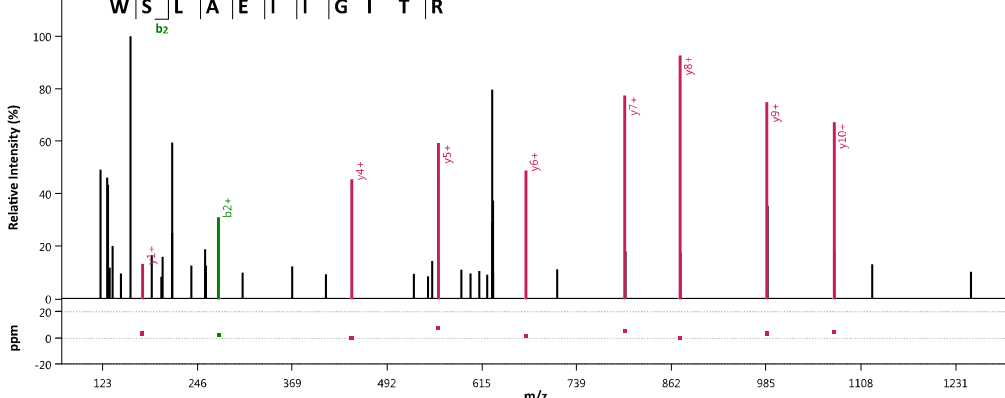

के
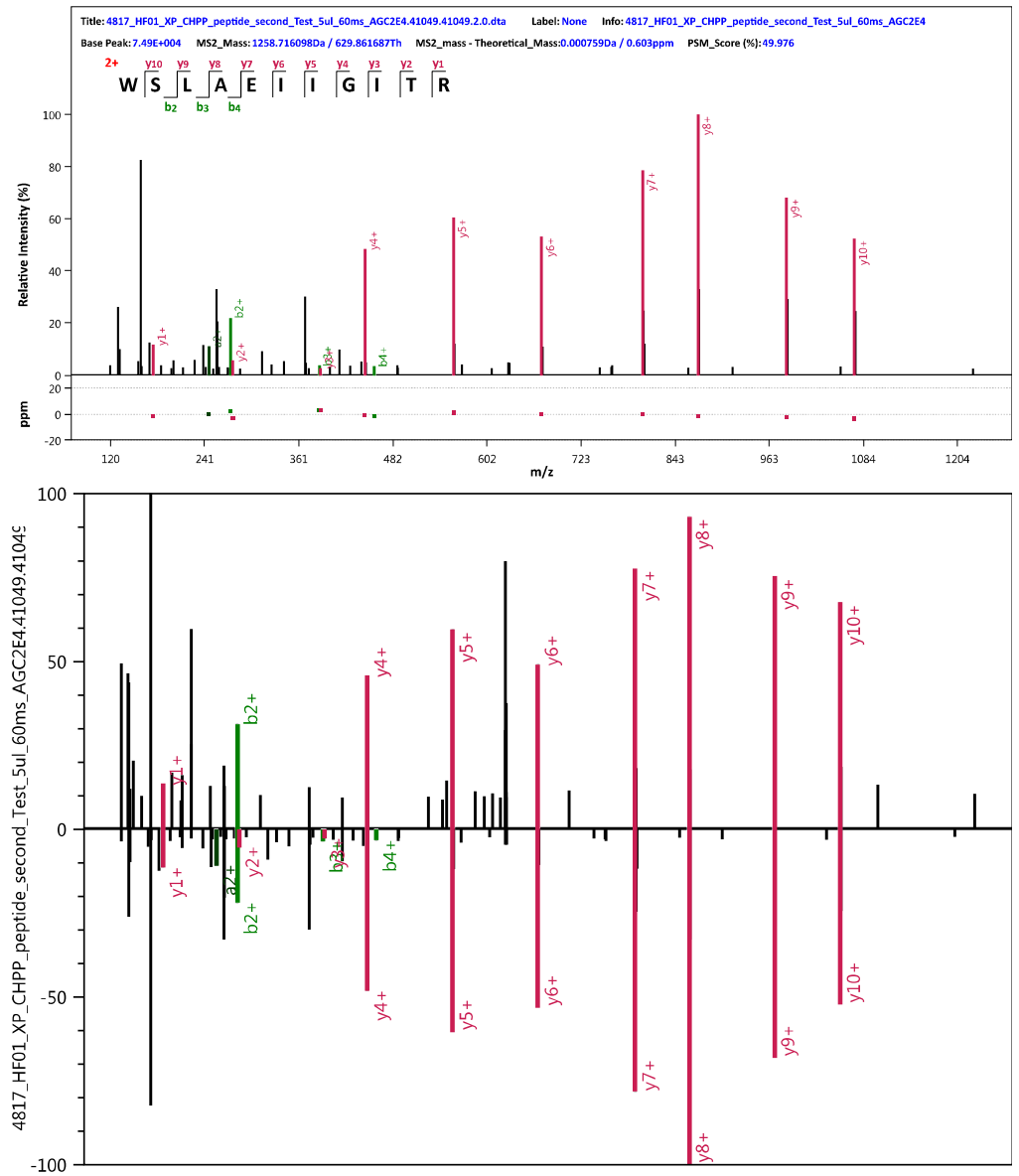
Titte: 4817_HF01_XP_CHPP_Has_SDS10_15_5Ul_60ms_AGC2E4.20690.20690.2.0.dta Label: None Info: 4817_HF01_XP_CHPP_Has_SDS10_15_5ul_60ms_AGC2EA Base Peak: 3.00E+005 MS2_Mass: 1350.705969Da / 675.856623Th MS2_mass - Theoretical_Mass:0.004828Da/ 3.574ppm PSM_Score (x):37.099

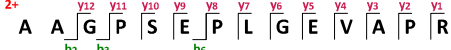

응

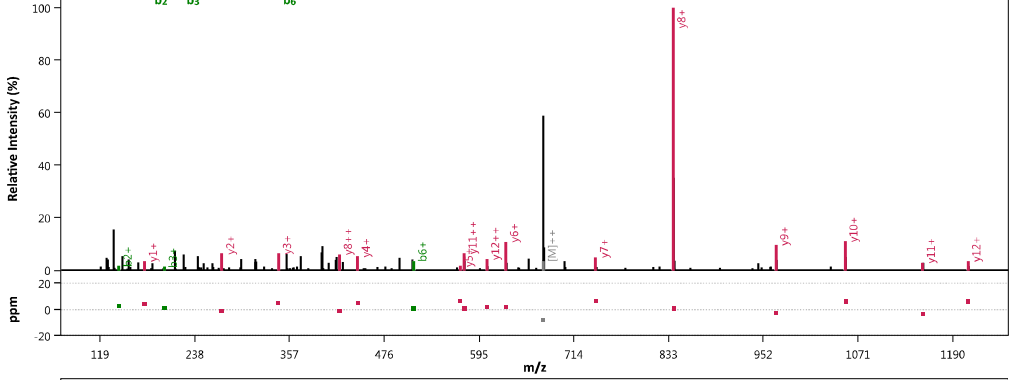

Title: 4817_HF01_XP_CHPP_peptide_second_Test_5ul_60ms_AGC2E4.17979.17979.20.dta Label: None Info: 4817_HF01_XP_CHPP_peptide_second_Test_5ul_60ms_AGC2E4 Base Peak: 2.91E+005 MS2_Mass: 1350.7017620a / 675.854519Th MS2_mass - Theoretical_Mass:0.000620Da / 0.459ppm PSM_Score (\%): 39.46

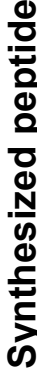

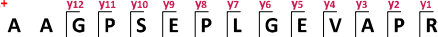
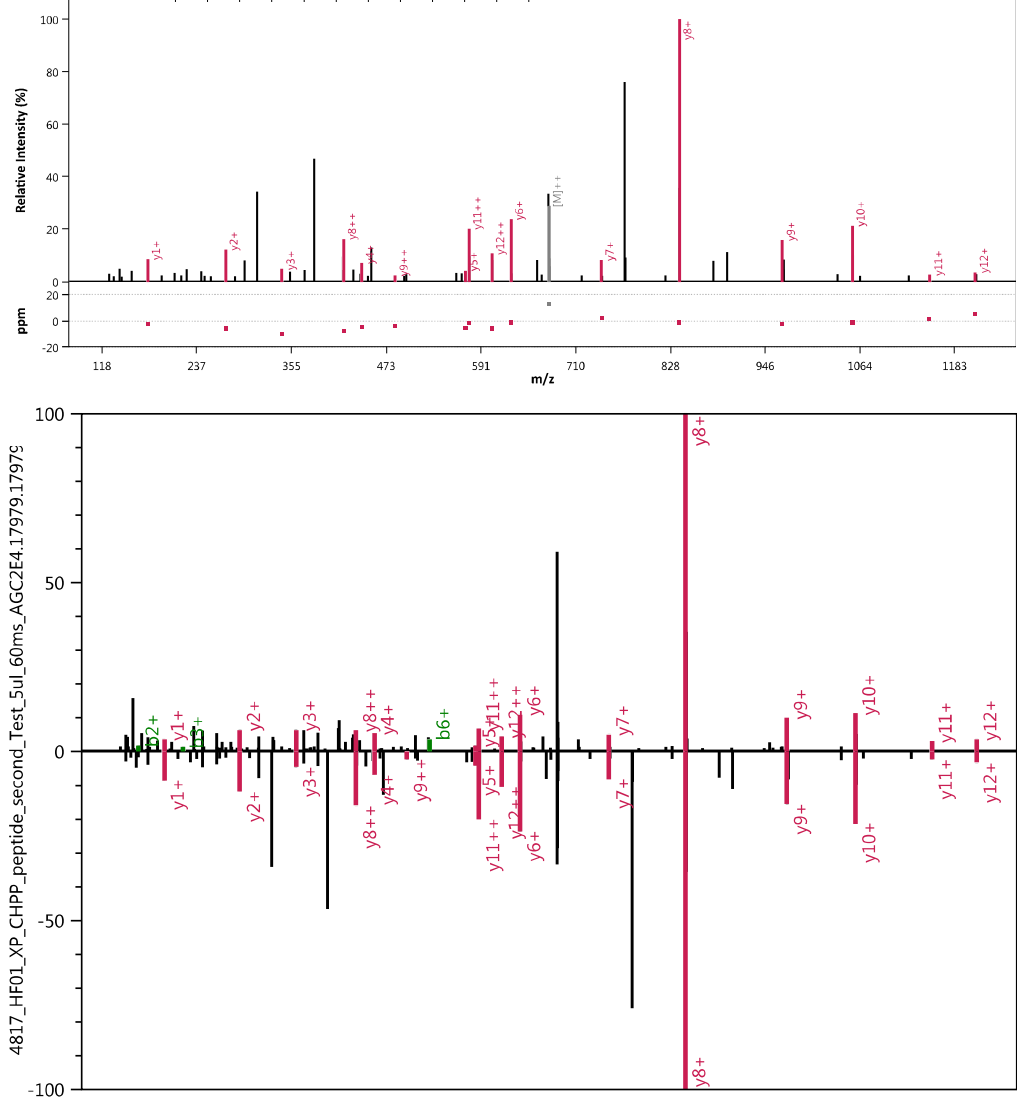
Title: 4817 _HF01_XP_CHPP_Has_SDS30_7_5ul_60ms_AGC2E4.37540.37540.3.0.dta Label: None Info: 4817_HF01_XP_CHPP_Has_SDS30_7_5ul_60ms_AGC2E4 Base Peak: 1.69E+005 MS2 Mass: 1208.712808Da/ 403.575787Th MS2 mass - Theoretical_Mass:0.001886Da/1.561ppm PSM_Score (\%):38.411

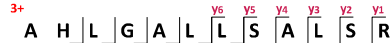

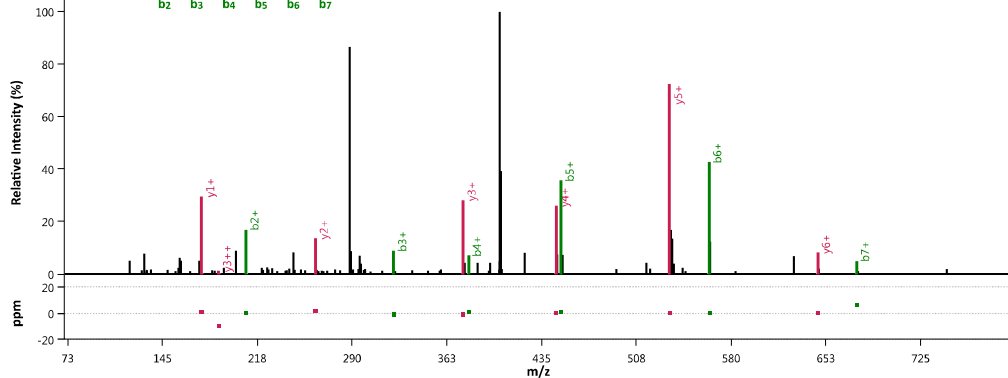

章
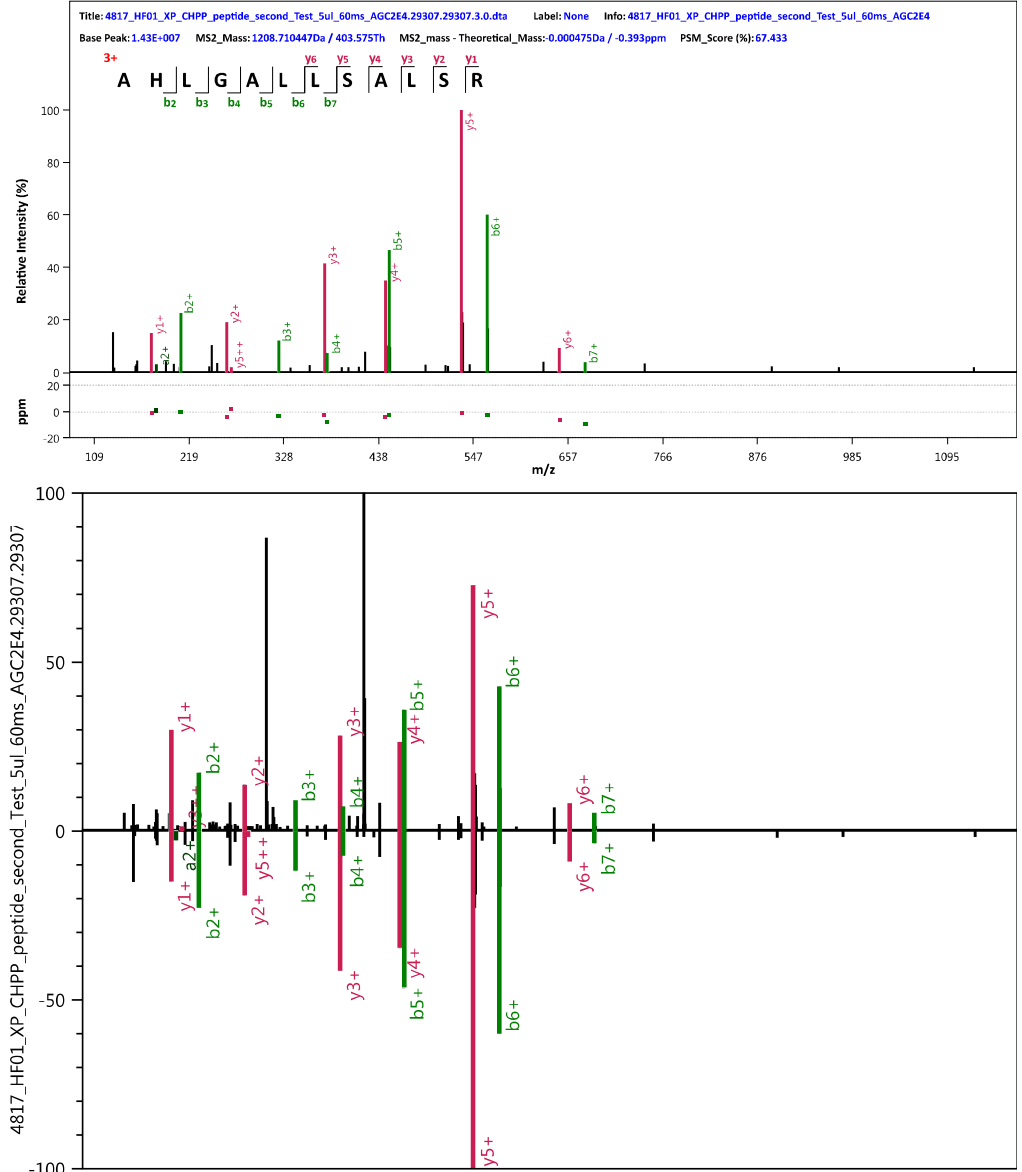


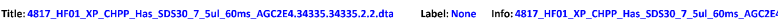
Base Peak: 1.32E+005 MS2_Mass: 1124.5597580 a / 562.783517Th MS2_mass - Theoretical_Mass: $0.0210710 \mathrm{Da} /-18.738 \mathrm{ppm}$ PSM_SCore (X): 29.112

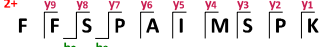

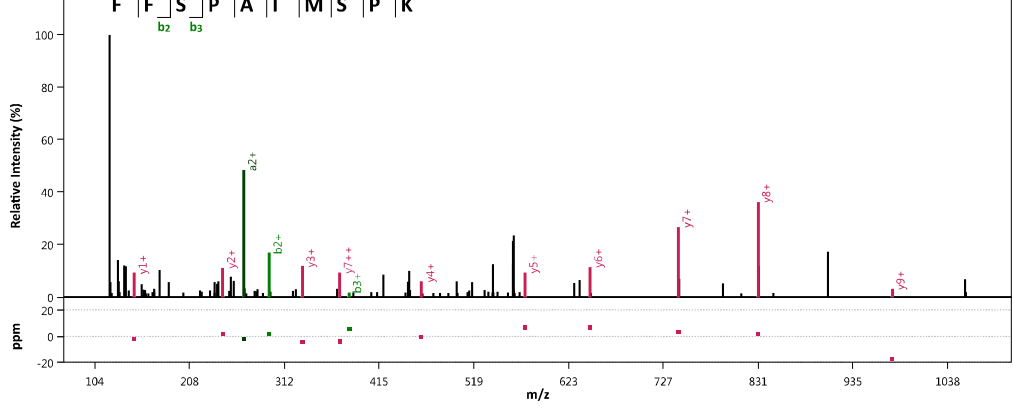

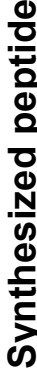
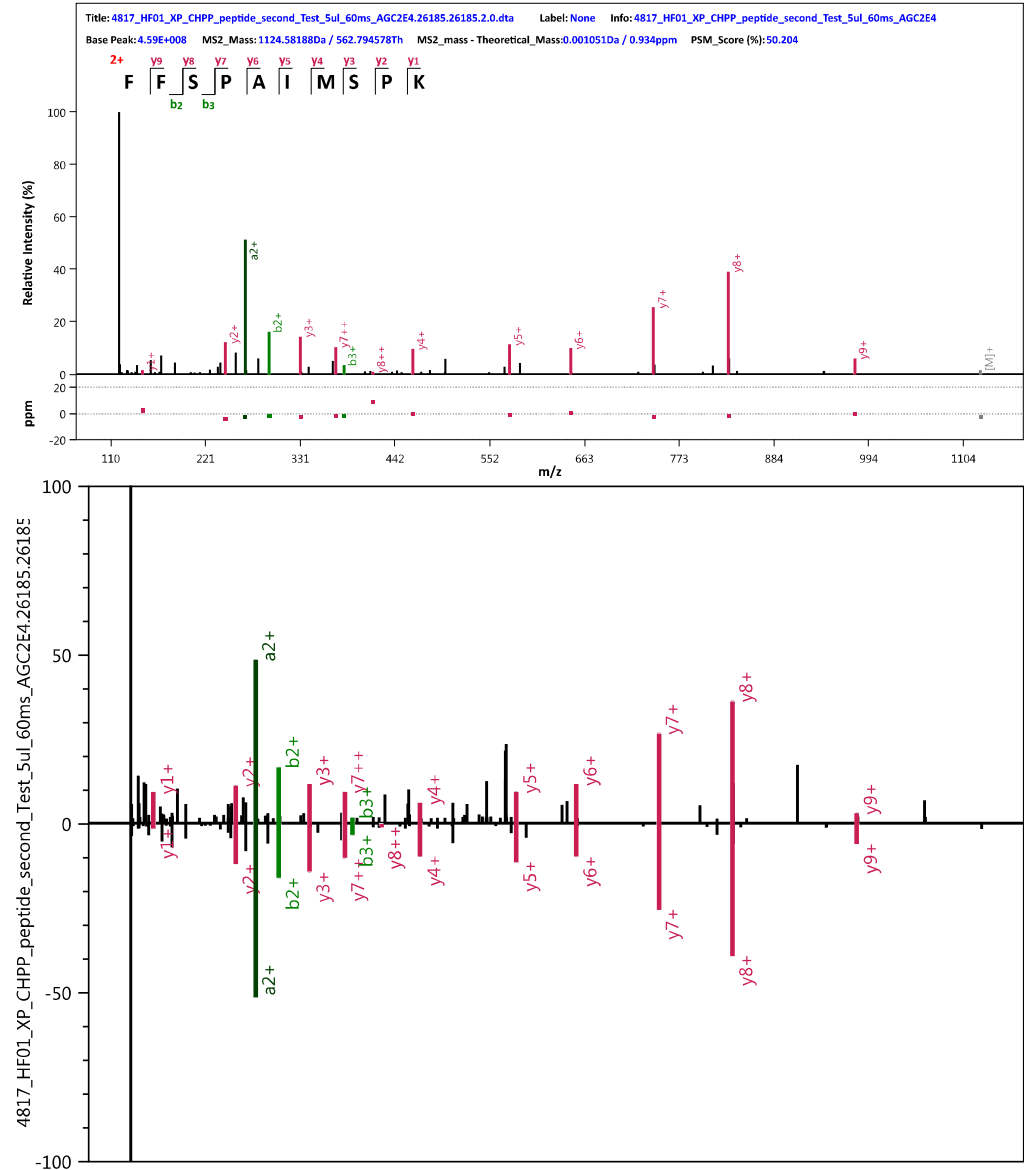

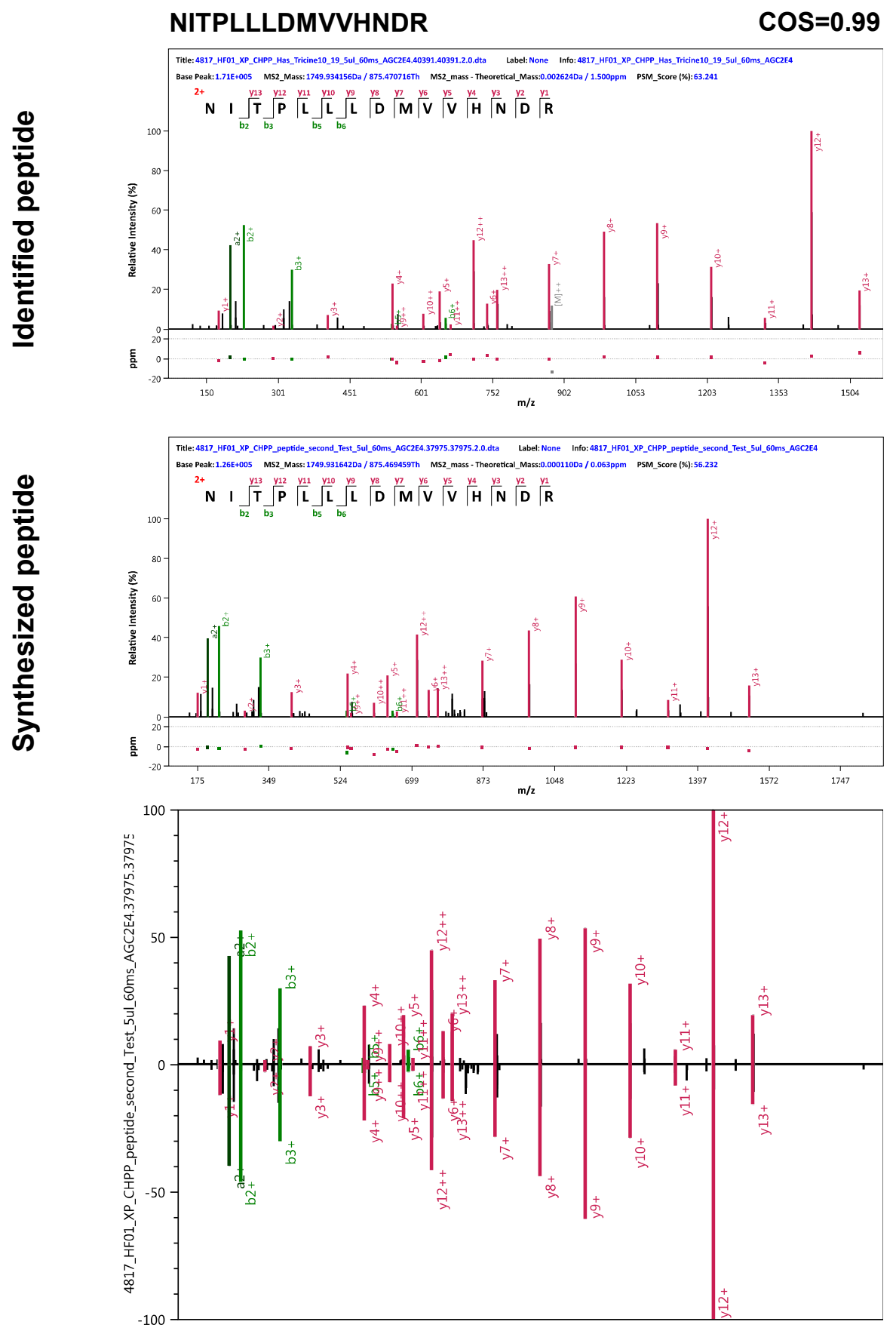

S-93 


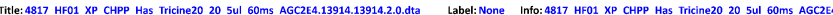
Base Peak: 5.35E+004 MS2 Mass: 1586.7807940a/ 793.894035Th MS2 mass - Theoretical_Mass:0.003980Da/2.508ppm PSM_Score (\$): 65.632

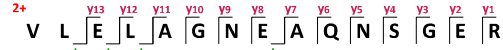

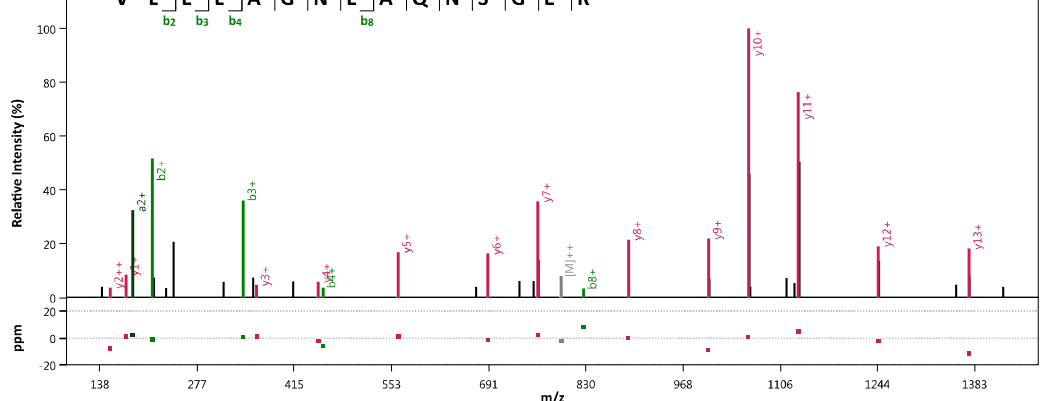

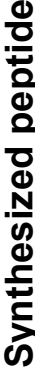

Title: 4817_HF01_XP_CHPP_peptide_second_Test_Sul_60ms_AGC2E4.12529.12529.2.0.dta Label: None Info:4817_HF01_XP_CHPP_peptide_second_Test_5ul_60ms_AGC2E Base Peak: 1.52E+005 MS2_Mass: 1586.776392Da/ 793.891834Th MS2_mass - Theoretical_Mass:-0.000422Da/-0.266ppm PSM_Score (\%): 60.234

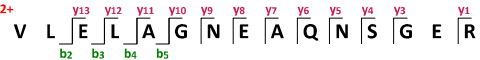
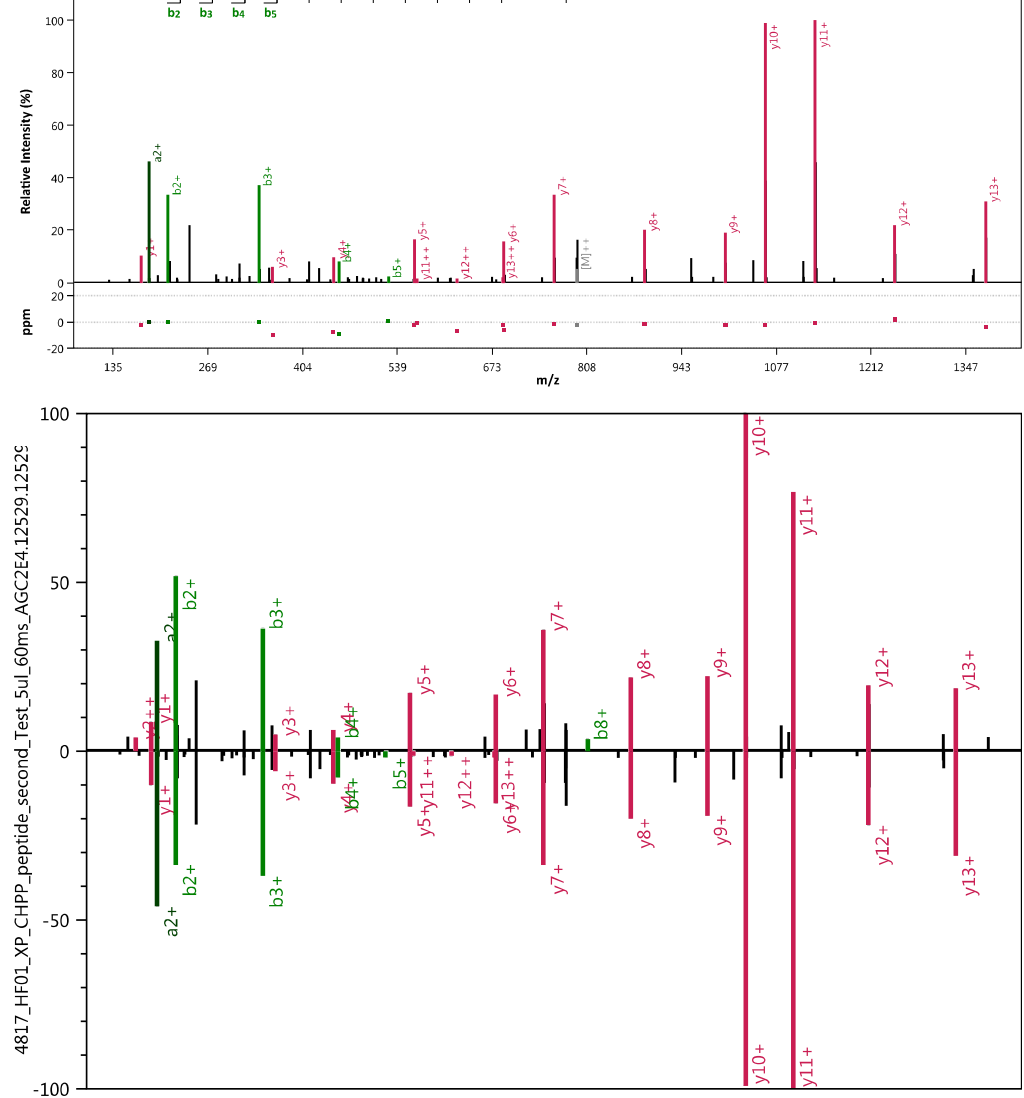
DGMMQKPGSNAPVGGNVTSNFSGDDLECR COS=0.95

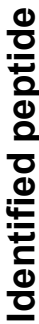

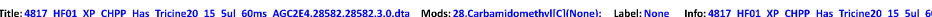
Base Peak: 1.01E+005 MS2_Mass: 3040.32136Da / 1014.111971Th MS2_mass - Theoretical_Mass:0.002153Da/0.708ppm PSM_Score (\%):40.238

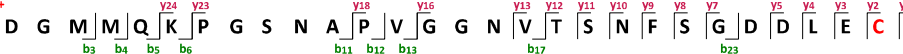

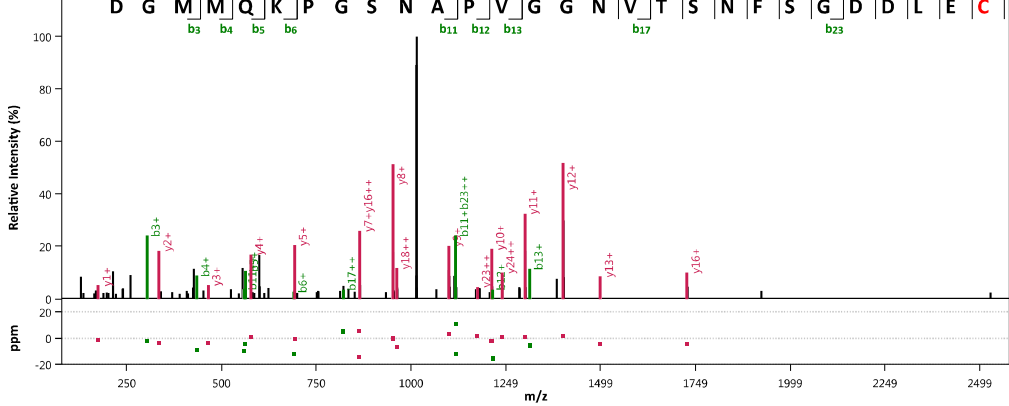

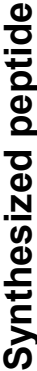
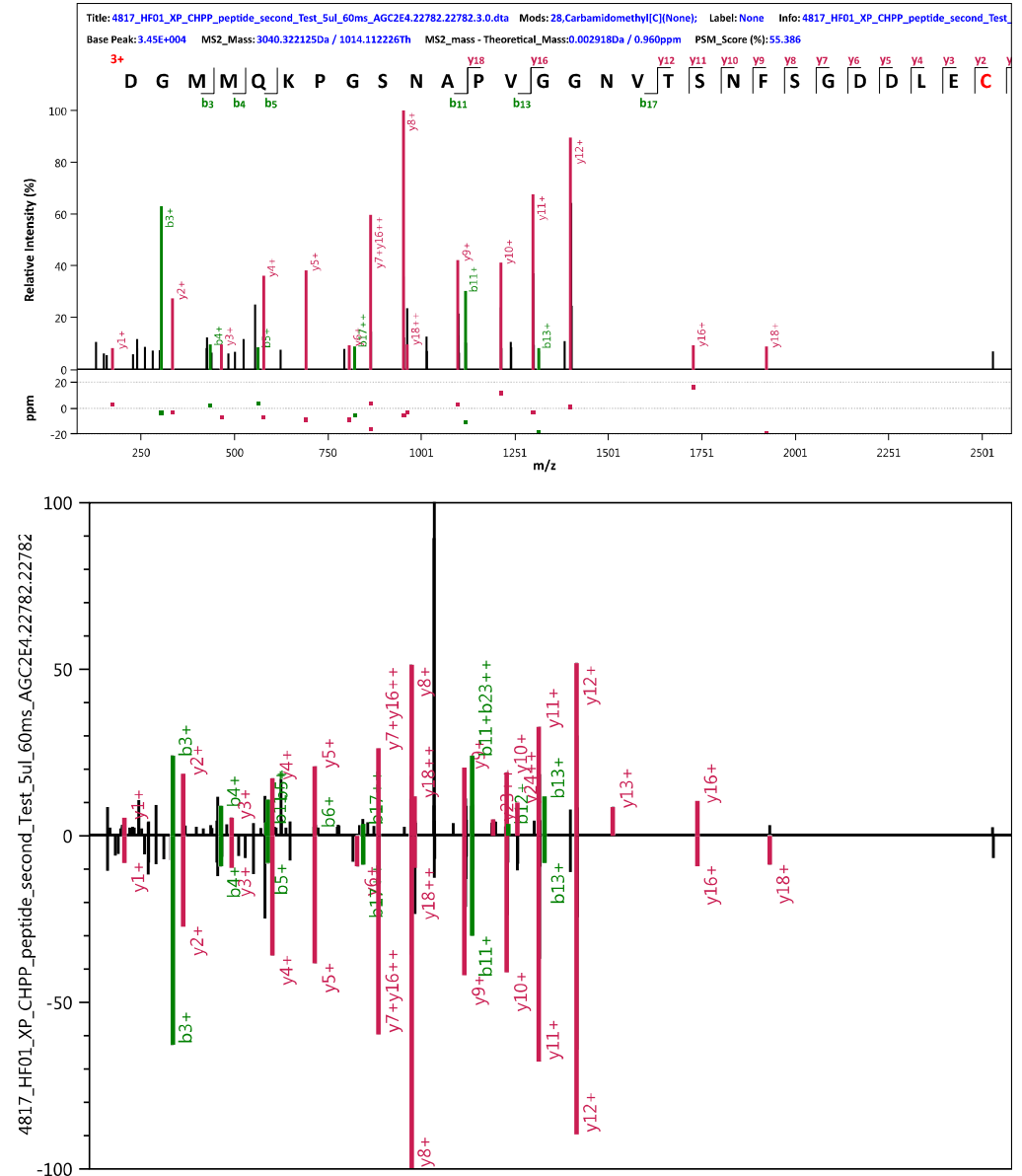

Base Peak:3.84E+004 MS2_Mass: 1145.58199Da / 573.294633Th MS2_mass - Theoretical_Mass:0.002351Da / 2.052ppm PSM_Score (\%): 70.147

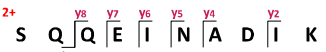

के

Title: 4817_HF01_XP_CHPP_peptide_second_Test_5ul_60ms_AGC2E4.9275.9275.2.0.dta Label:None Info: 4817_HF01_XP_CHPP_peptide_second_Test_5ul_60m5_AGC2E4

源

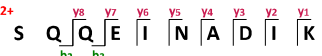
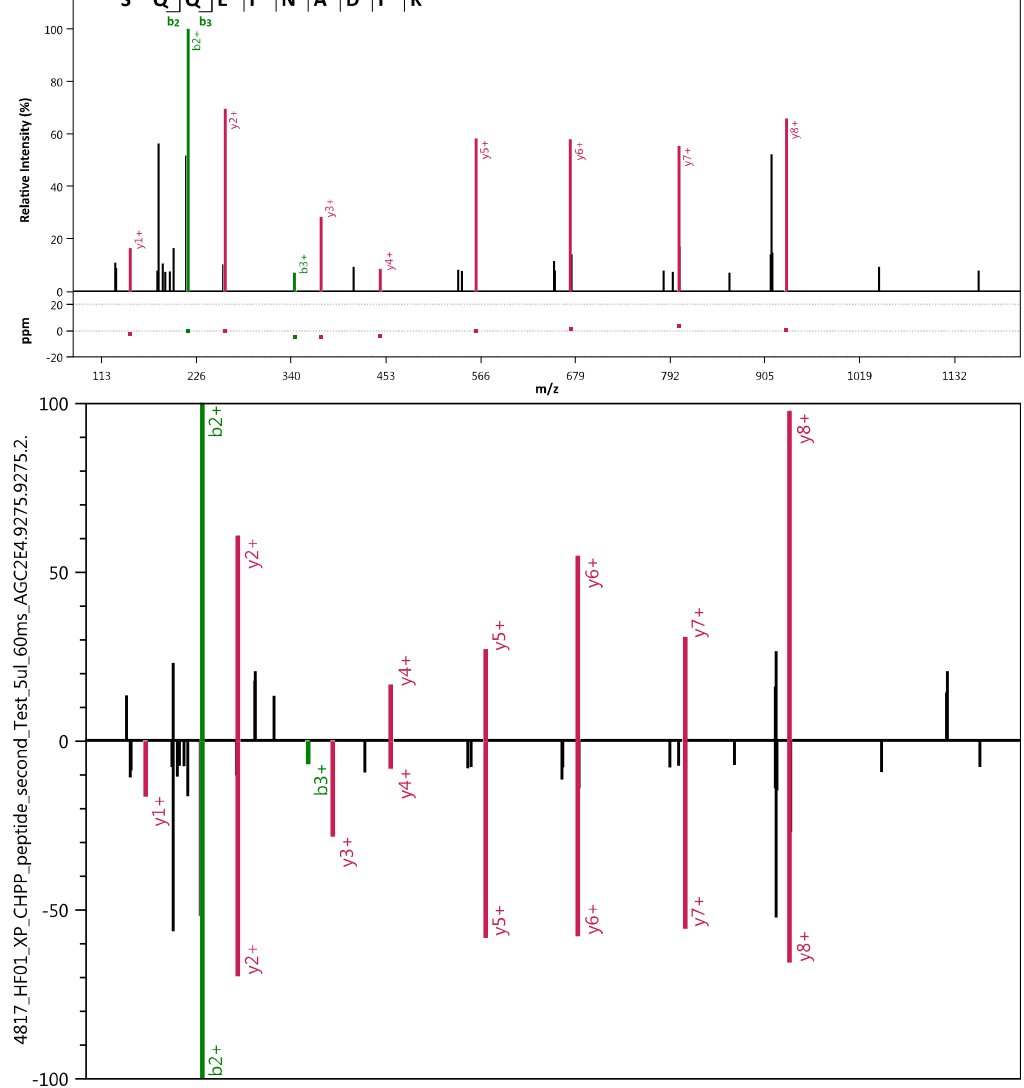
Base Peak: 7.40E+004 MS2_Mass: 1627.919112Da / 814.463194Th MS2_mass - Theoretical_Mass:0.002572Da/ 1.580ppm PSM_Score (\%):19.502

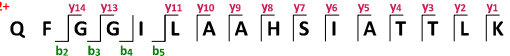

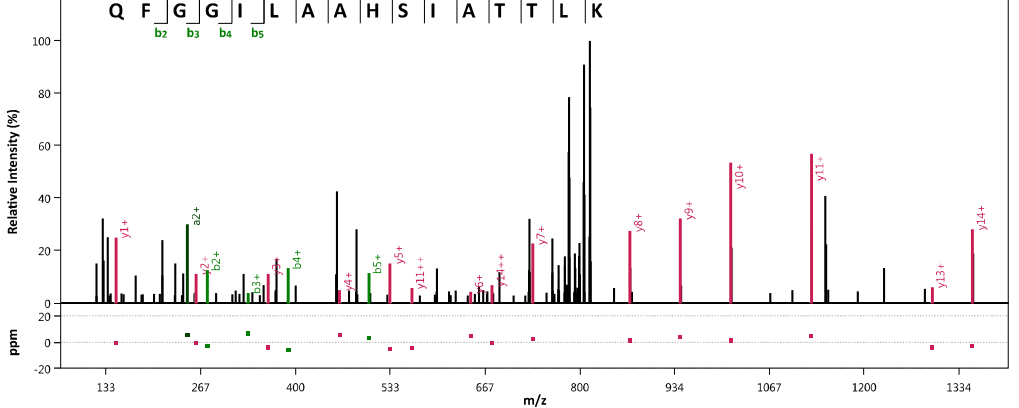

के

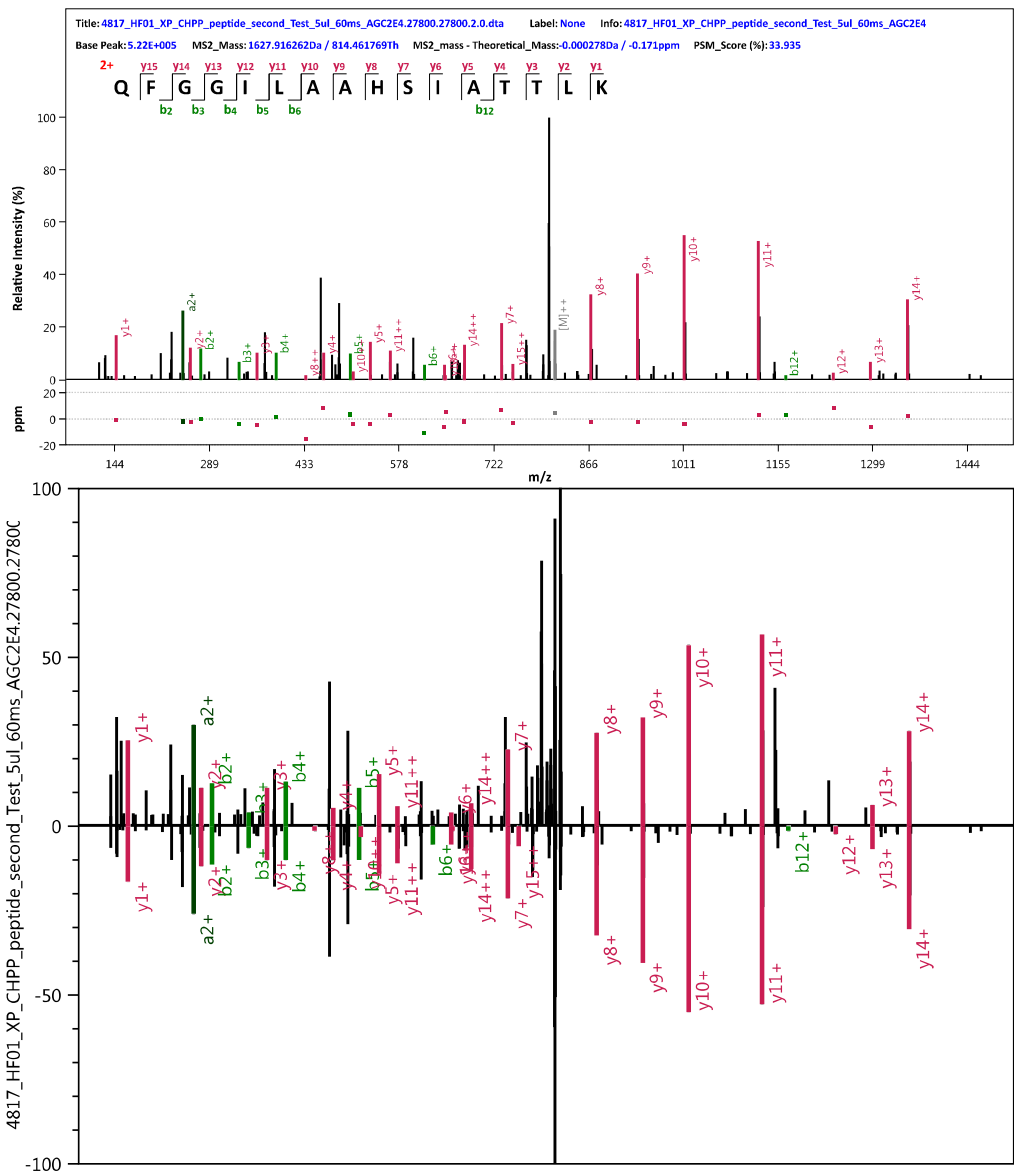


Title: 4817_HF01_XP_CHPP_Has_SDS30_12_Sul_60ms_AGC2E4.36860.36860.2.2.dta Label: None Info: 4817_HF01_XP_CHPP_Has_SDS30_12_5Ul_60ms_AGC2E4 Base Peak:1.35E+005 MS2_Mass: 1626.908212Da / 813.957744Th MS2_mass - Theoretical_Mass:0.002179Da/1.340ppm PSM_Score (\%): 35.304

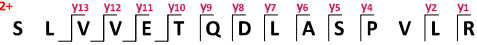

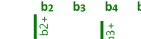

竞

le: 4817_HF01_XP_CHPP_peptide_second_Test_5ul_60 ms_AGC2E4.31481.31481.2.0.dta Label: None Info: 4817_HF01_XP_CHPP_peptide_second_Test_5ul_60ms_AGC2E4 Base Peak:9.21E+004 MS2_Mass: 1626.905758Da/813.956517Th MS2_mass - Theoretical_Mass:-0.000275Da/-0.169ppm PSM_Score (\%): 56.585

के

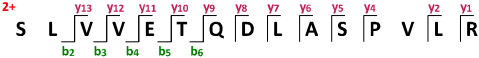
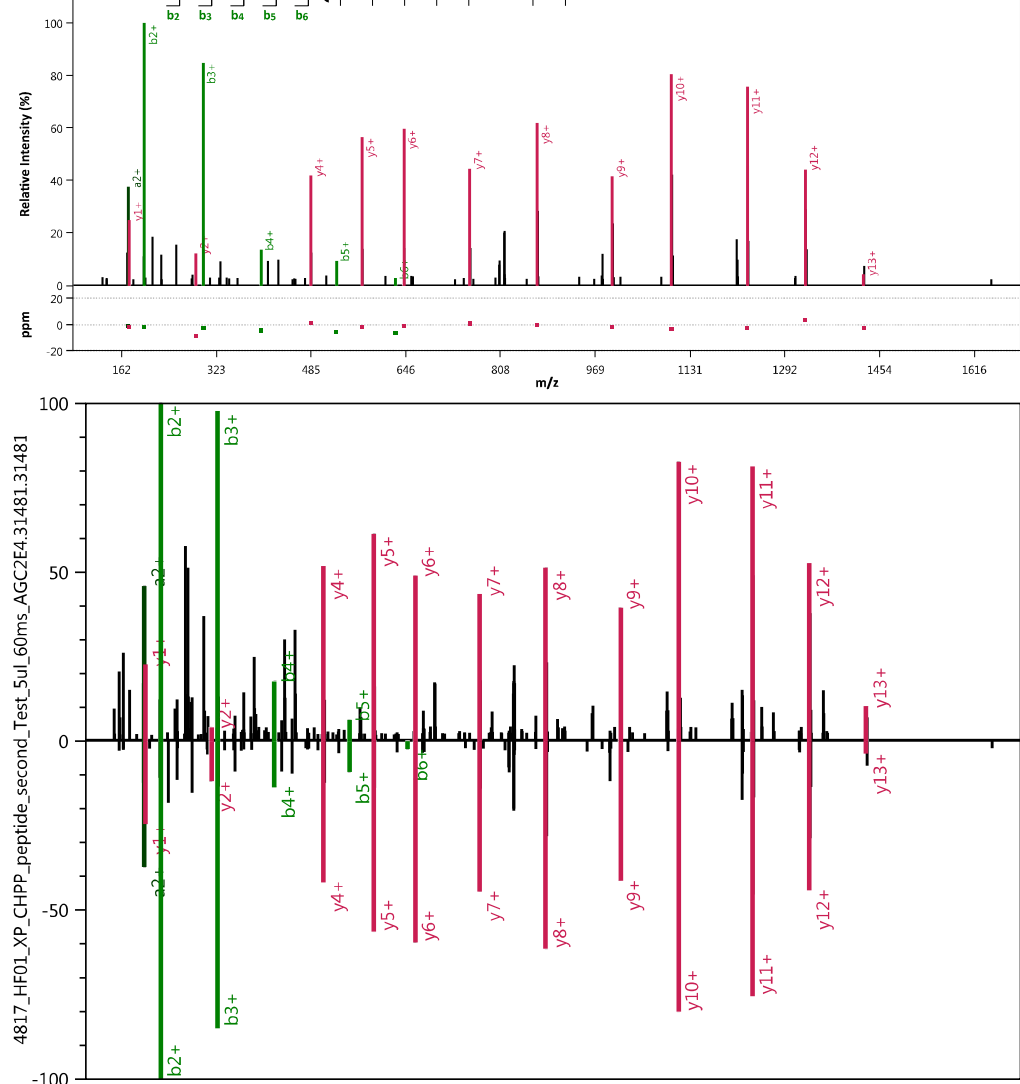
Titte: 4817_HF01_XP_CHPP_Has_SDS30_6_5ul_60ms_AGC2E4.21720.21720.2.0.dta Label: None Info: 4817_HF01_XP_CHPP_Has_SDS30_6_5ul_60ms_AGC2EA

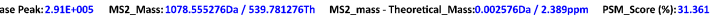

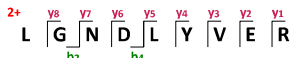

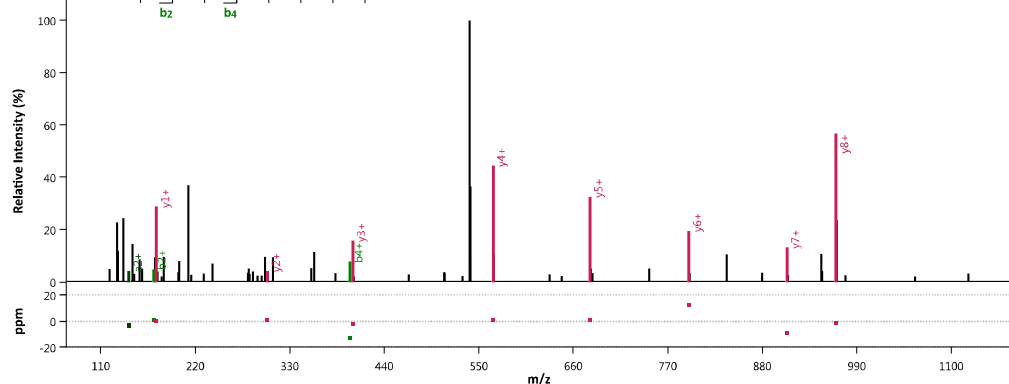

के

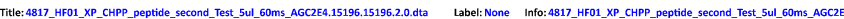
Base Peak:2.91E+008 MS2_Mass: 1078.553616Da / 539.780446Th MS2_mass - Theoretical_Mass:0.000915Da / 0.849ppm PSM_Score (\%): 60.26

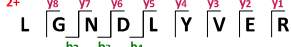
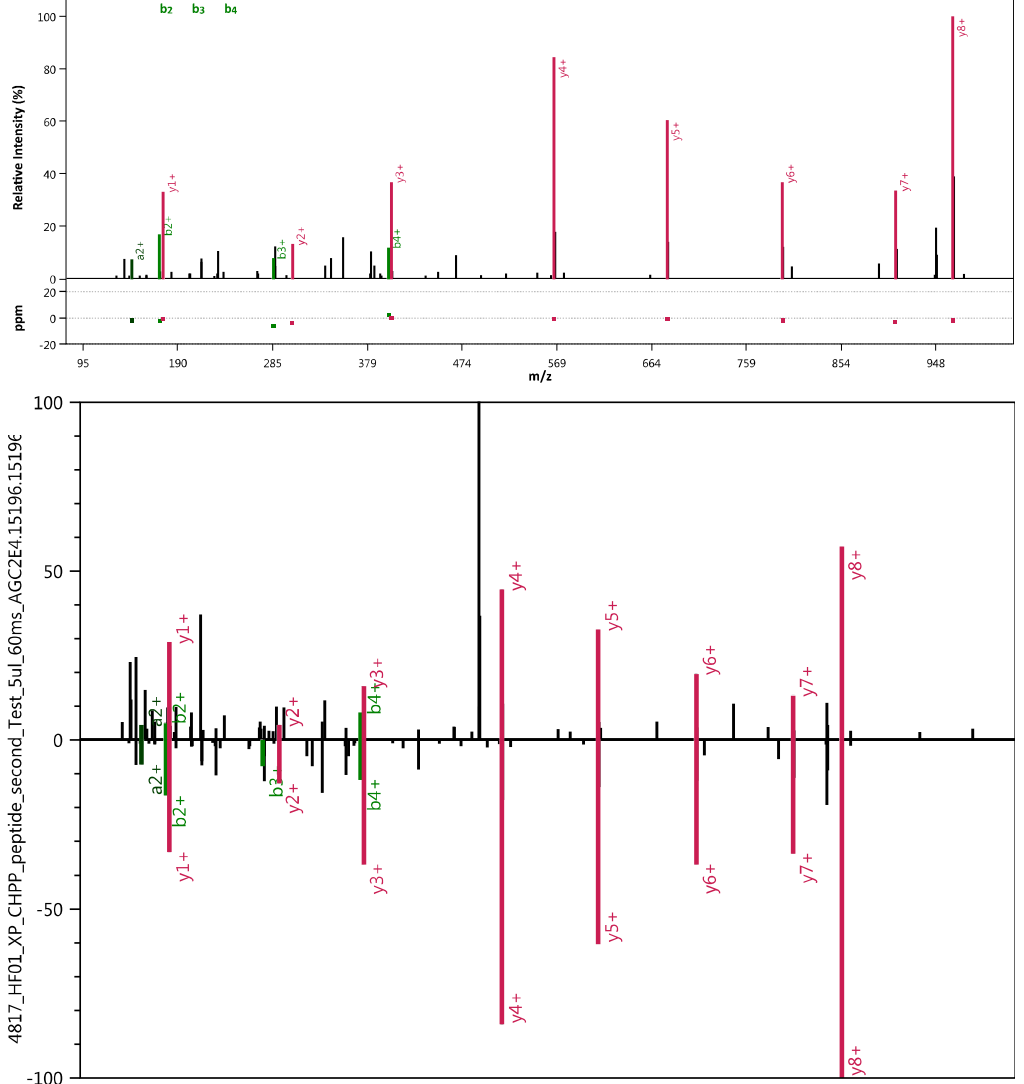
Titile: CHPP_TESTIS_Tricine_3002.30489.30489.2.1.dta Label: None Info:CHPP_TESTIS_Tricine_3002

Peak:4.26E+003 M52 Mass: 1863.937468Da / 932.472372Th M52 mass - Theorticin _N

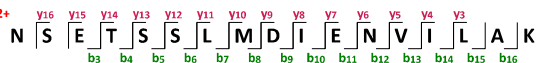

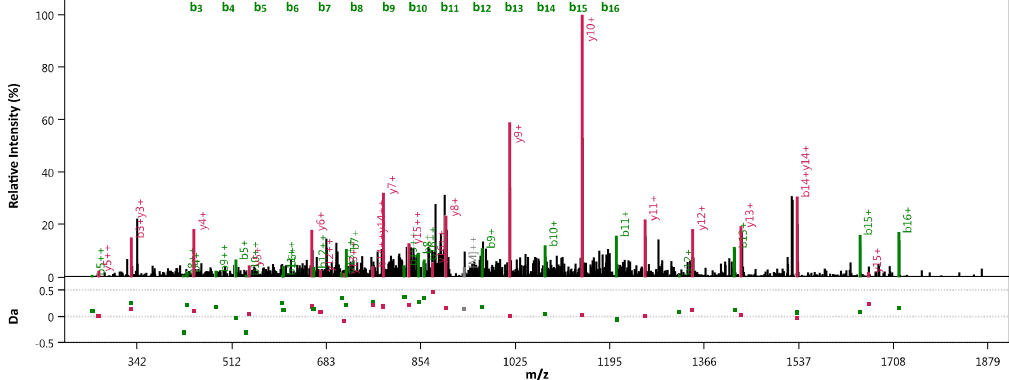

क्ष
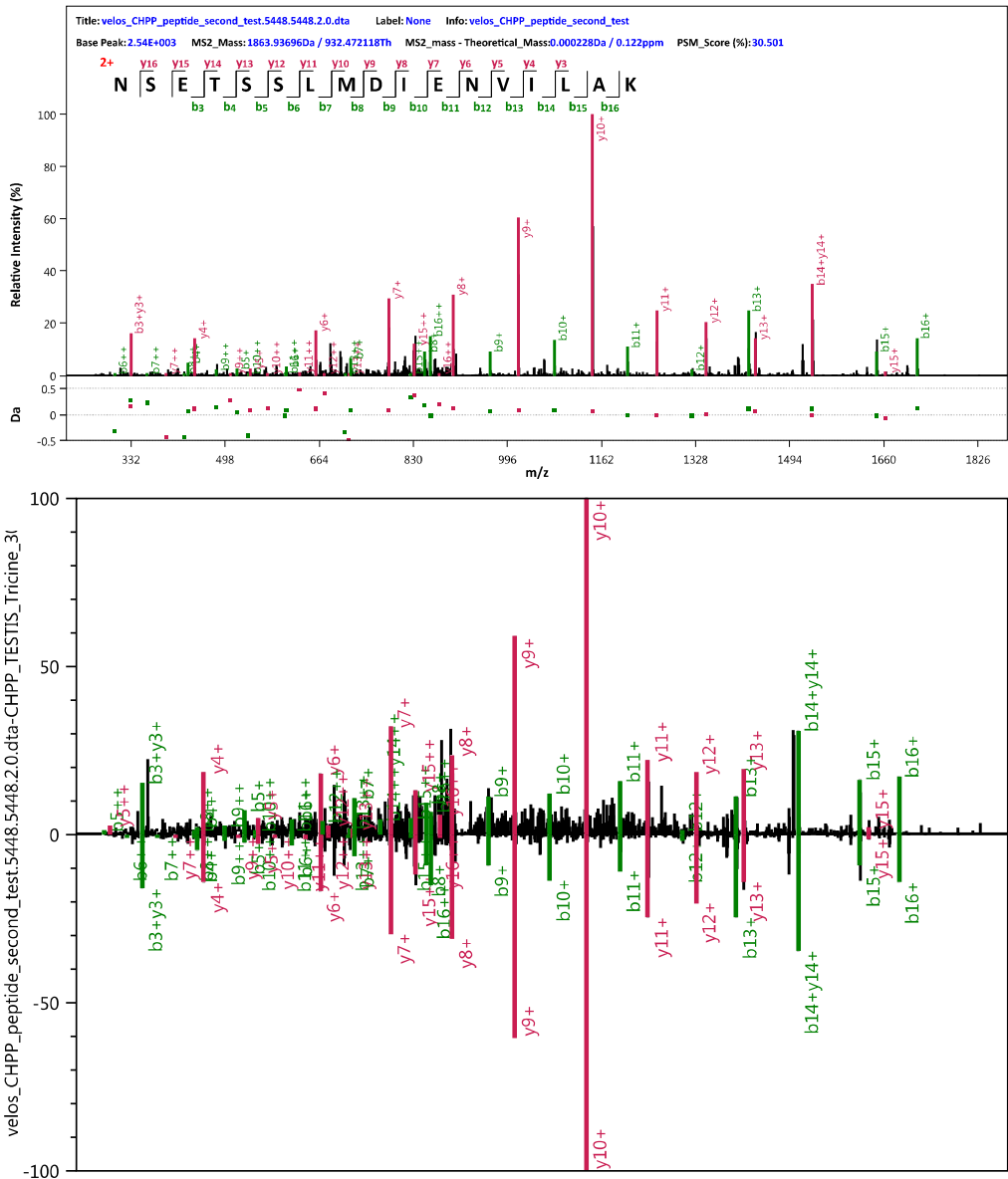


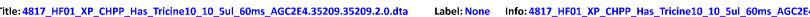
Base Peak:2.17E+005 MS2_Mass: 1881.916319Da / 941.461798Th MS2_mass-Theoretical_Mass:0.003414Da / 1.814ppm PSM_Score (X): 30.339

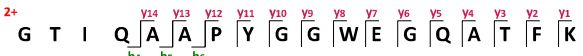

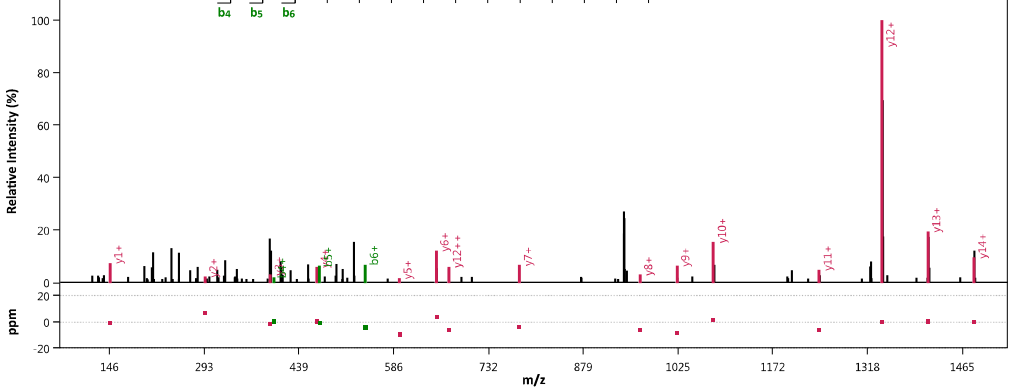

Title: 4817_HF01_XP_CHPP_peptide_second_Test_5ul_60ms_AGC2E4.41442.41442.2.0.dta Label: None Info: 4817_HF01_XP_CHPP__eptide_second_Test_5ul_60ms_AGC2E4 Base Peak: 1.68E+005 MS2_Mass: 1881.914062Da / 941.460669Th MS2_mass - Theoretical_Mass:0.001156Da/0.614ppm PSM_Score (\%):36.217

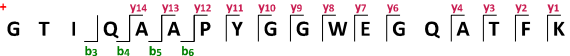
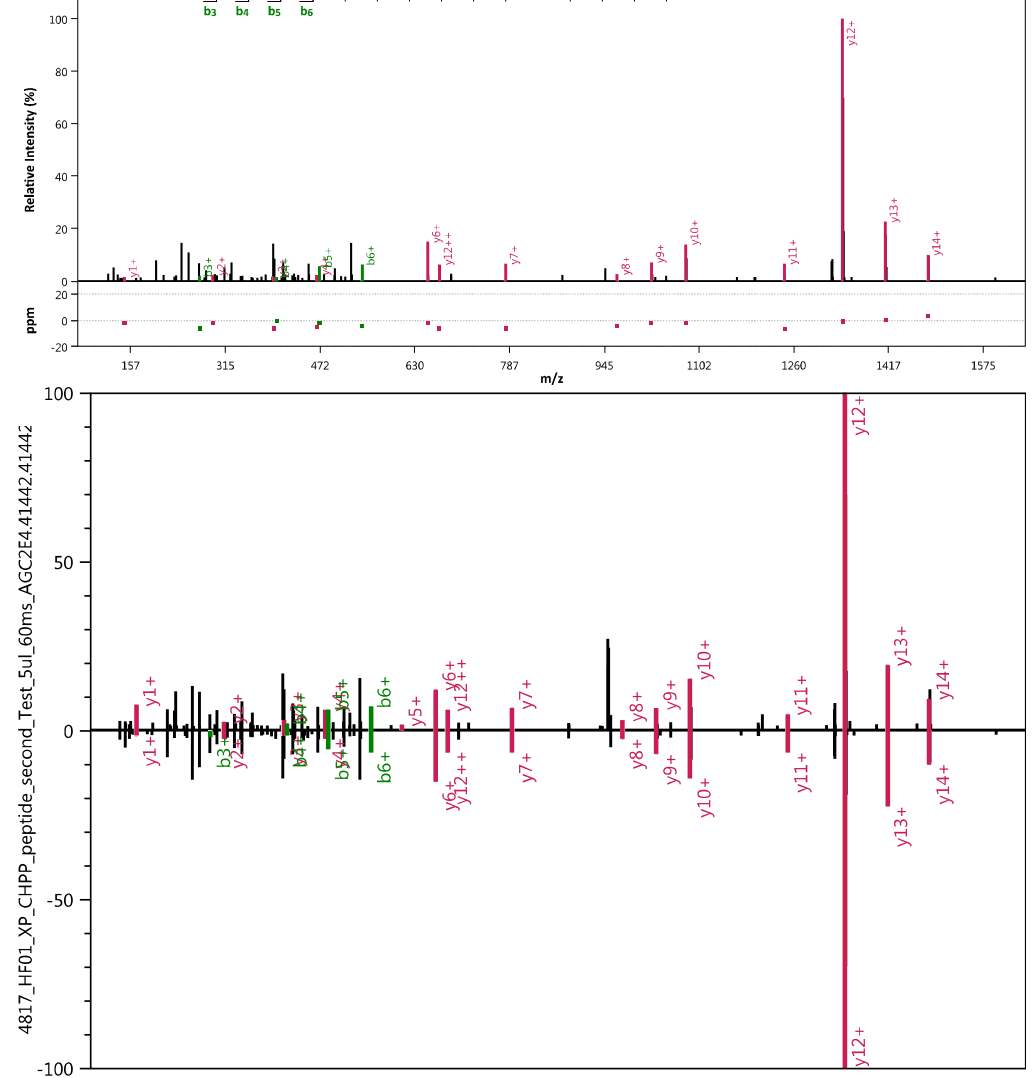


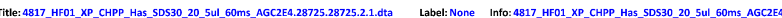
Base Peak:2.53E+005 MS2_Mass: 1273.657502Da / 637.332389Th MS2_mass - Theoretical_Mass:0.0040340a / 3.167ppm PSM_Score (\%): 45.724

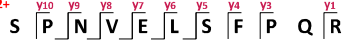

के

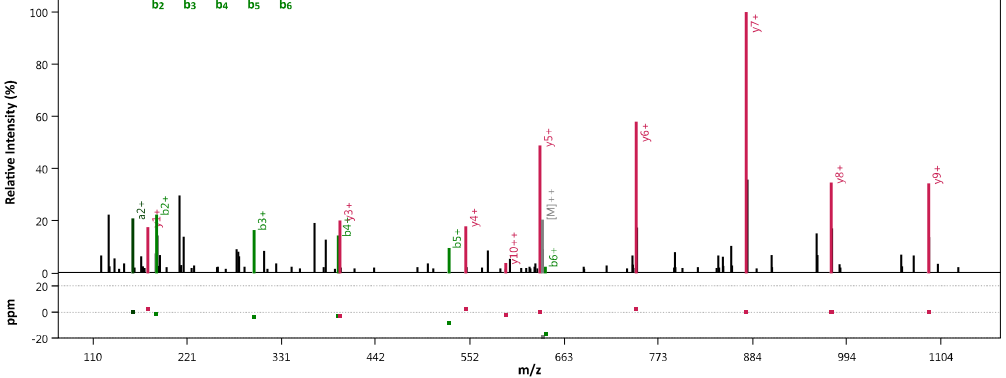

Title: 4817_HF01_XP_CHPP_peptide__eccond_Test_5ul_60ms_AGC2E4.22301.22301.2.0.dta Label: None Info: 4817_HF01_XP_CHPP_peptide_second_Test_SUI_60ms_AGC2E4

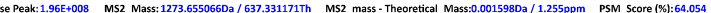

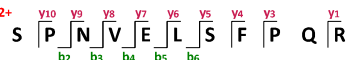
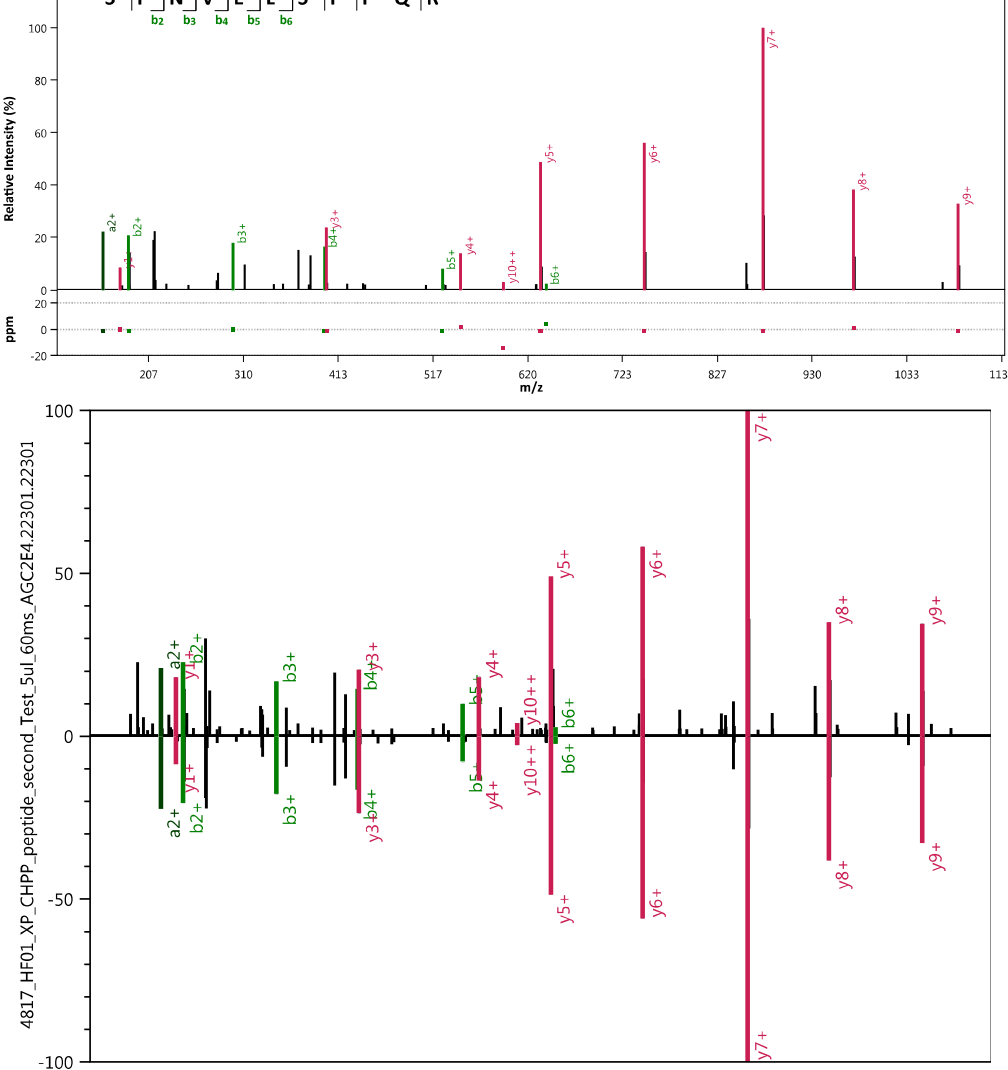
Title:4817_HF01_XP_CHPP_Has_Tricine10_10_Sul_60ms_AGC2E4.37896.37896.2.0.dta Mods: 10,Carbamidomethy|[C]|(None): Label: None Info: 4817_HF01_XP_CHPP_Has_Tricine10_10_5ul_60 Base Peak: 2.22E+005 MS2_Mass: 1316.689878Da / 658.848577Th MS2_mass - Theoretical_Mass:0.002075Da / 1.576ppm PSM_Score (\%):41.293

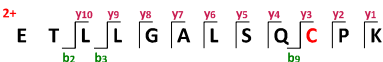

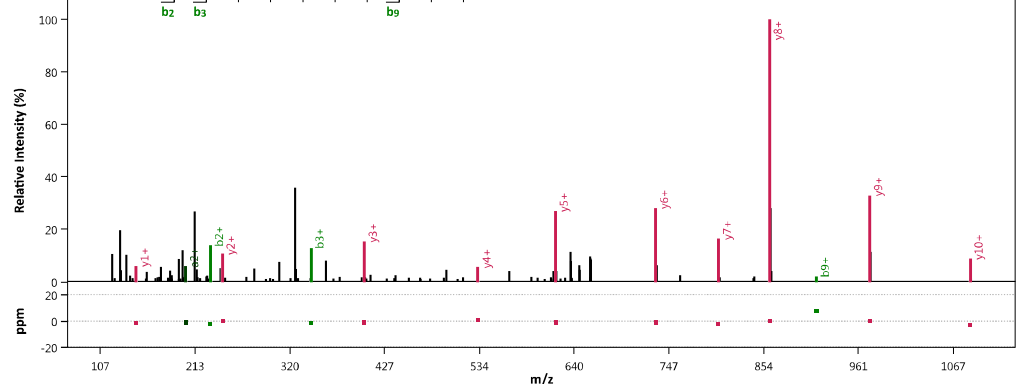

Title: 4817_HF01_XP_CHPP_peptide_second_Test_5ul_60ms_AGC2E4.28220.28220.2.0.dta Mods: 10,Carbamidomethyl[C](None); Label: None Info: 4817_HF01_XP_CHPP_peptide_second_Test Base Peak:1.23E+007 MS2_Mass: 1316.689364Da / 658.84832Th MS2_mass - Theoretical_Mass:0.001561Da / 1.185ppm PSM_Score (\%): 50.886

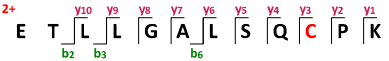

के
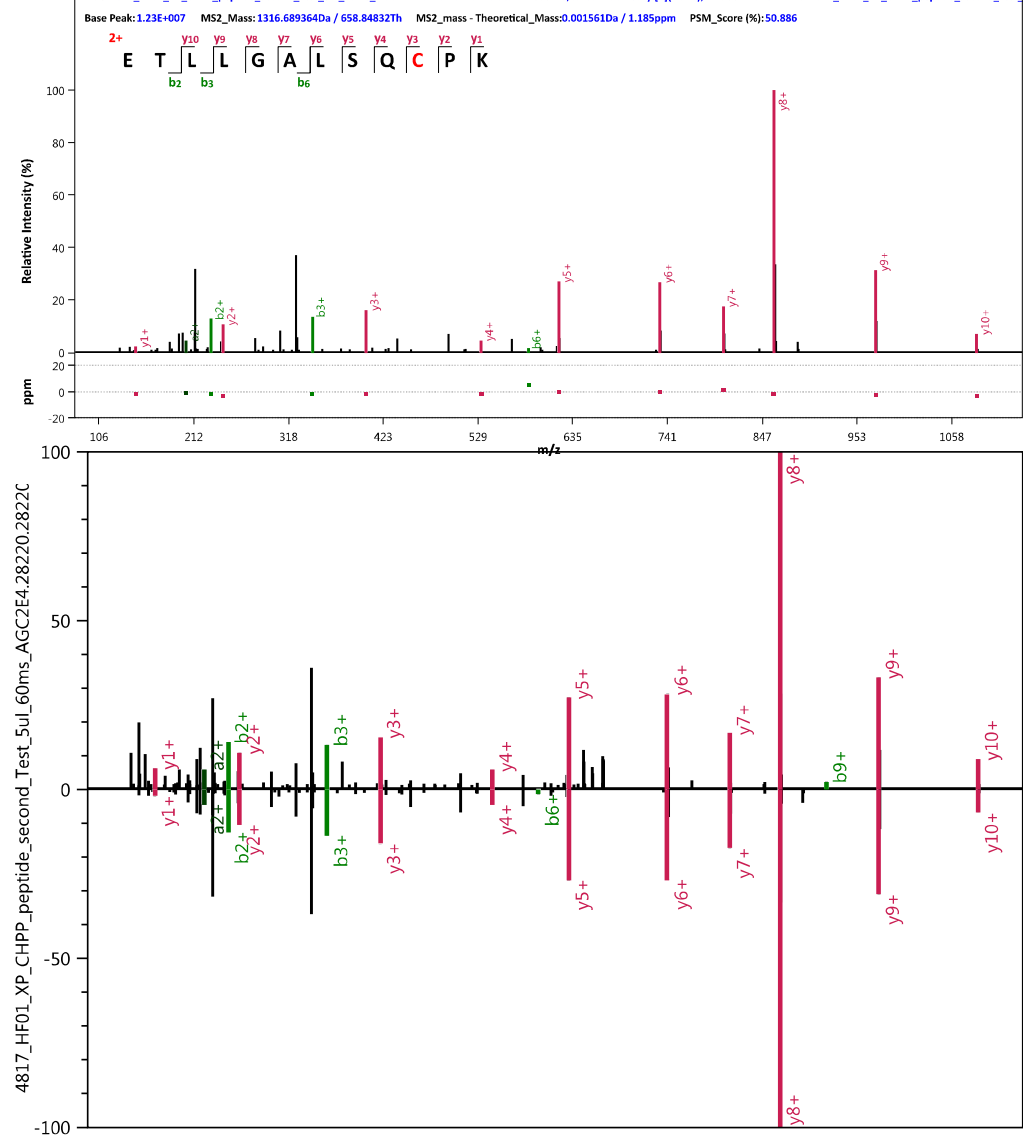
Titele:4817_HF01_XP_CHPP_Has_Tricine10_10_5ul_60ms_AGC2E4.5997.5997.2.0.dta Label: None Info: 4817_HF01_XP_CHPP_Has_Tricine10_10_5ul_60ms_AGC2E4 Base Peak: 4.37E+004 MS2_Mass: 1440.710549Da / 720.858913Th MS2_mass - Theoretical_Mass:0.002870Da / 1.992ppm PSM_SCore (\%):46.965

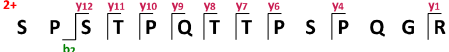

2

d

$100-$

(n)

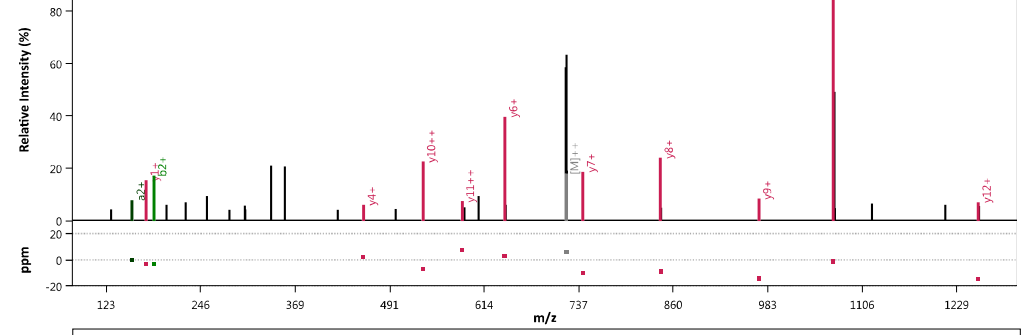

Title: 4817_HF01_XP_CHPP_peptide_second_Test_5ul_60ms_AGC2E4.4277.4277.2.0.dta Label: None Info: 4817_HF01_XP_CHPP_peptide_second_Test_5ul_60ms_AGC2E4 Base Peak: 1.85E+007 MS2_Mass: 1440.706554Da / 720.856915Th MS2_mass - Theoretical_Mass:-0.001126Da /-0.781ppm PSM_Score (\%): 57.109

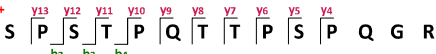
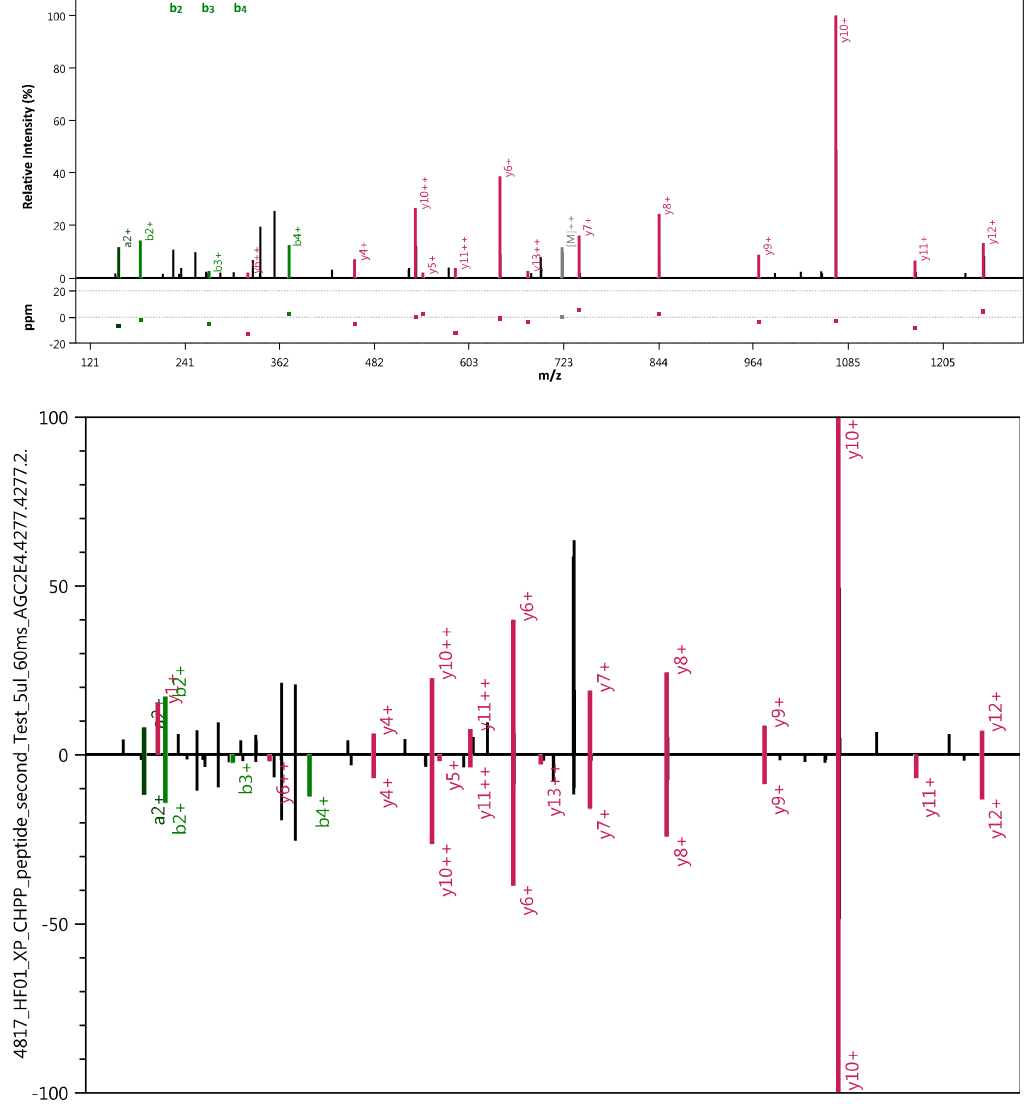


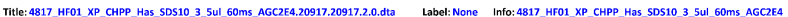
Base Peak:6.69E+004 MS2_Mass:1632.802030a / 816.904653Th MS2_mass - Theoretical_Mass:0.004481Da / 2.744ppm PSM_Score (\%):39.324

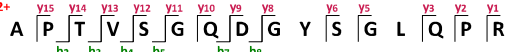

के Base Peak:2.04E+008 MS2_Mass: 1632.797276Da/816.902276Th MS2_mass - Theoretical_Mass:-0.000273Da/-0.167ppm PSM_Score (\%): 64.629

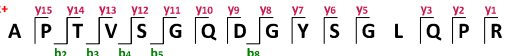
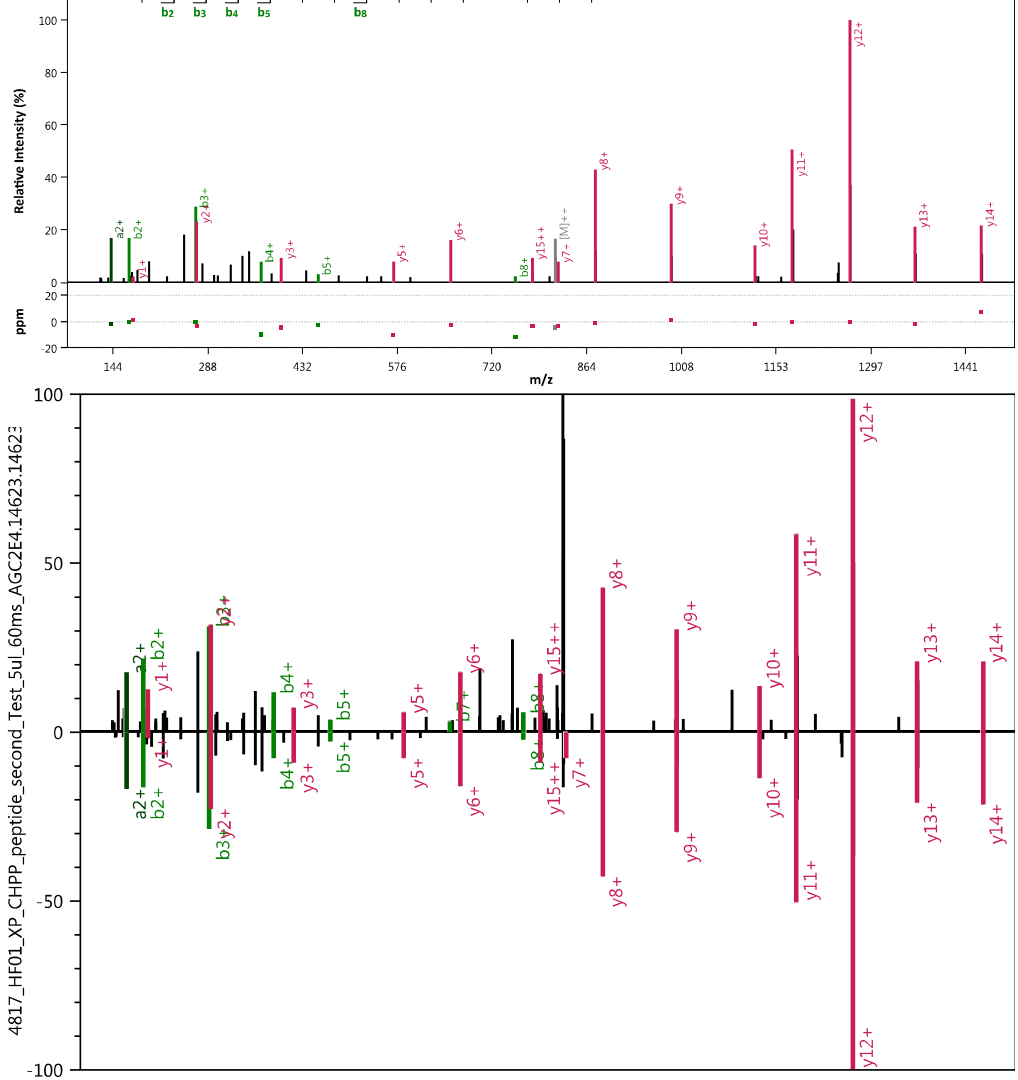


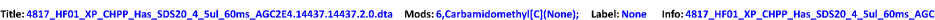
Base Peak:9.03E+004 MS2_Mass: 1310.514668Da / 655.760972Th MS2_mass - Theoretical_Mass:0.004092Da / 3.122ppm PSM_Score (\%): 48.848

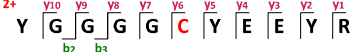

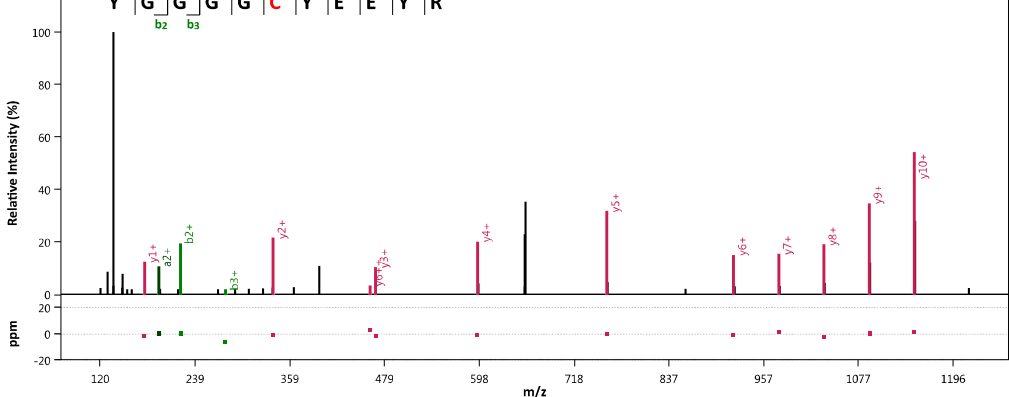

Title: 4817_HF01_XP_CHPP__eptide_second_Test_5Ul_60ms_AGC2E4.10804.10804.2.0.dta Mods: 6,Carbamidomethy|[C](None); Label: None Info:4817_HF01_XP_CHPP_peptide_second_Test_5 Base Peak:5.14E+005 MS2_Mass:1310.510296Da / 655.758786Th MS2_mass - Theoretical_Mass:-0.000280Da/-0.214ppm PSM_Score (\%):53.782

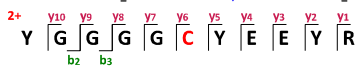
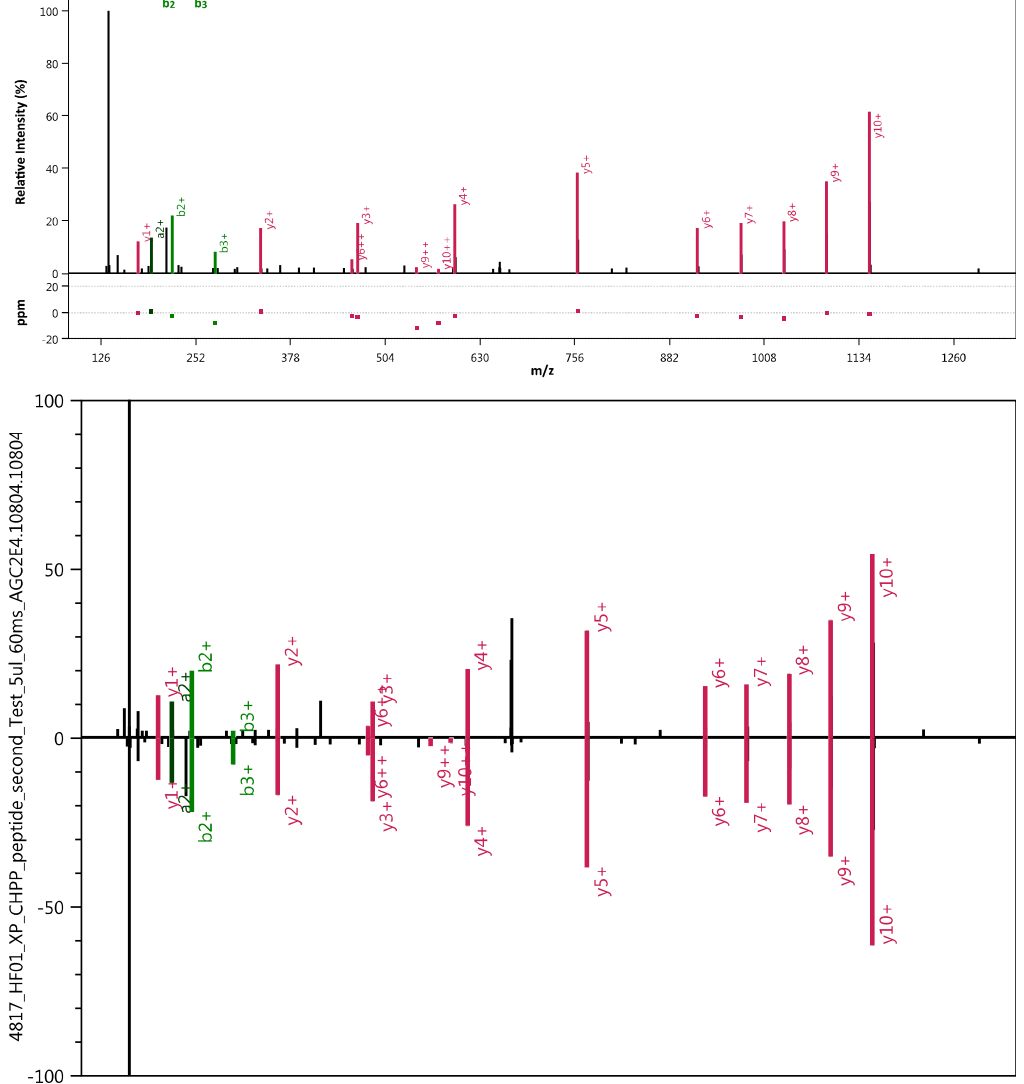
ELAENLGVTEDKVR

$\cos =0.97$

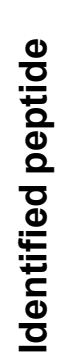

Title: 4817_HF01_XP_CHPP_Has_SDS10_22_SUL__60ms_AGC2E4.21258.21258.2.0.dta Label:None Info: 4817_HF01_XP_CHPP_Has_SDS10_22_5ul_60ms_AGC2E4 Base Peak: 4.64E+004 MS2_Mass: 1572.825814Da/ 786.916545Th MS2_mass - Theoretical_Mass:0.003113Da/1.979ppm PSM_Score (\%): 59.762

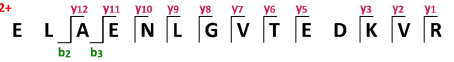
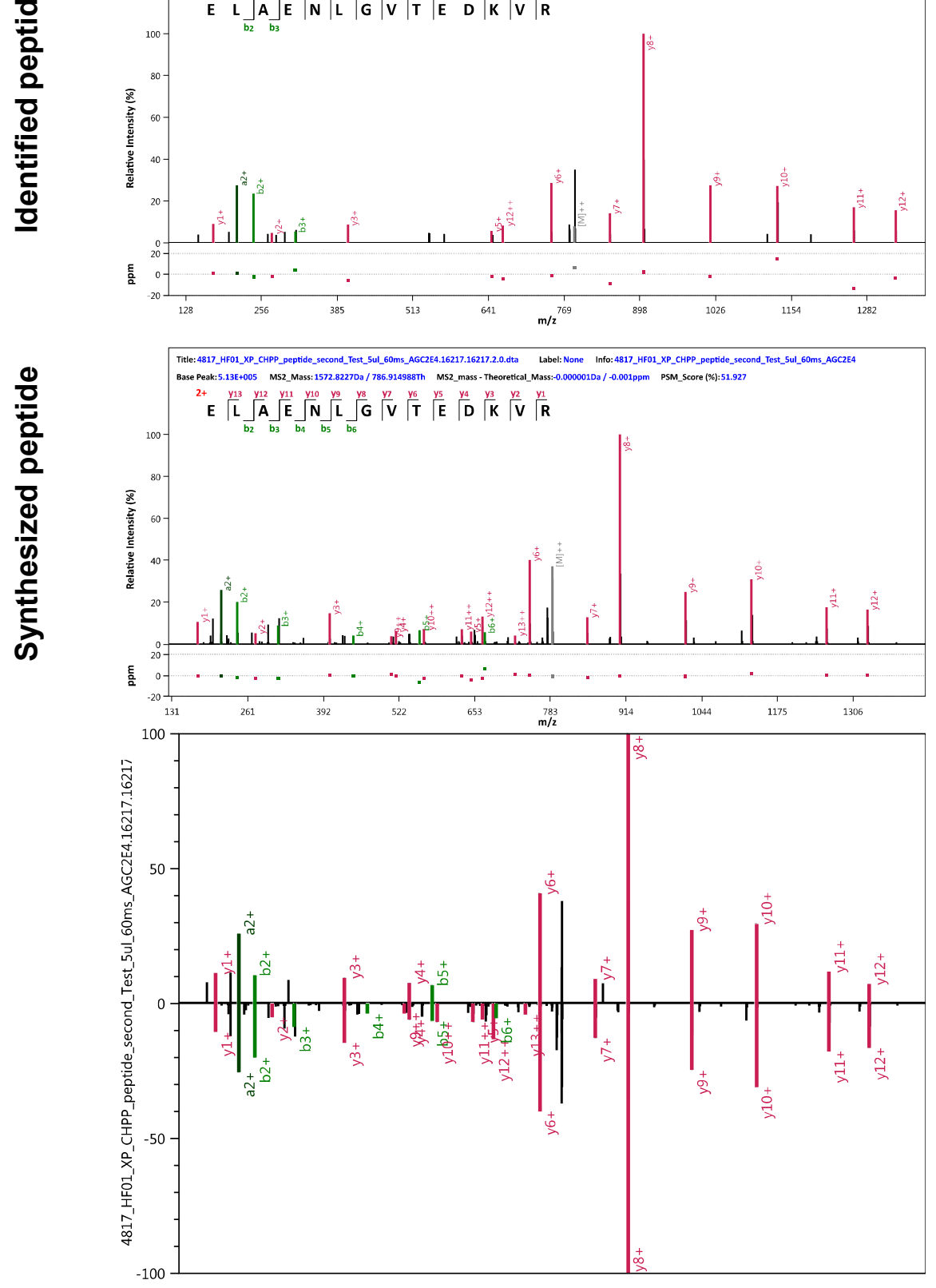

S-107 
Tite: CHPP_Testis__SDS_3023.31128.31128.2.0.dta Label: None Info:CHPP_Testis_SDS_3023

ase Peak:7.33E+003 MS2_Mass: 1709.915502Da / 855.461389Th MS2_mass - Theoretical_Mass:0.004717Da / 2.759ppm PSM_Score (7): 23.832

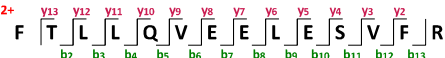

웜

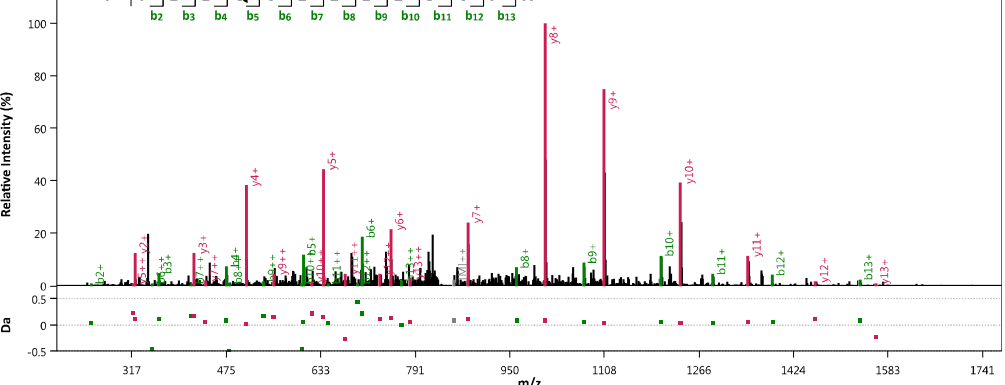

क्ष̃
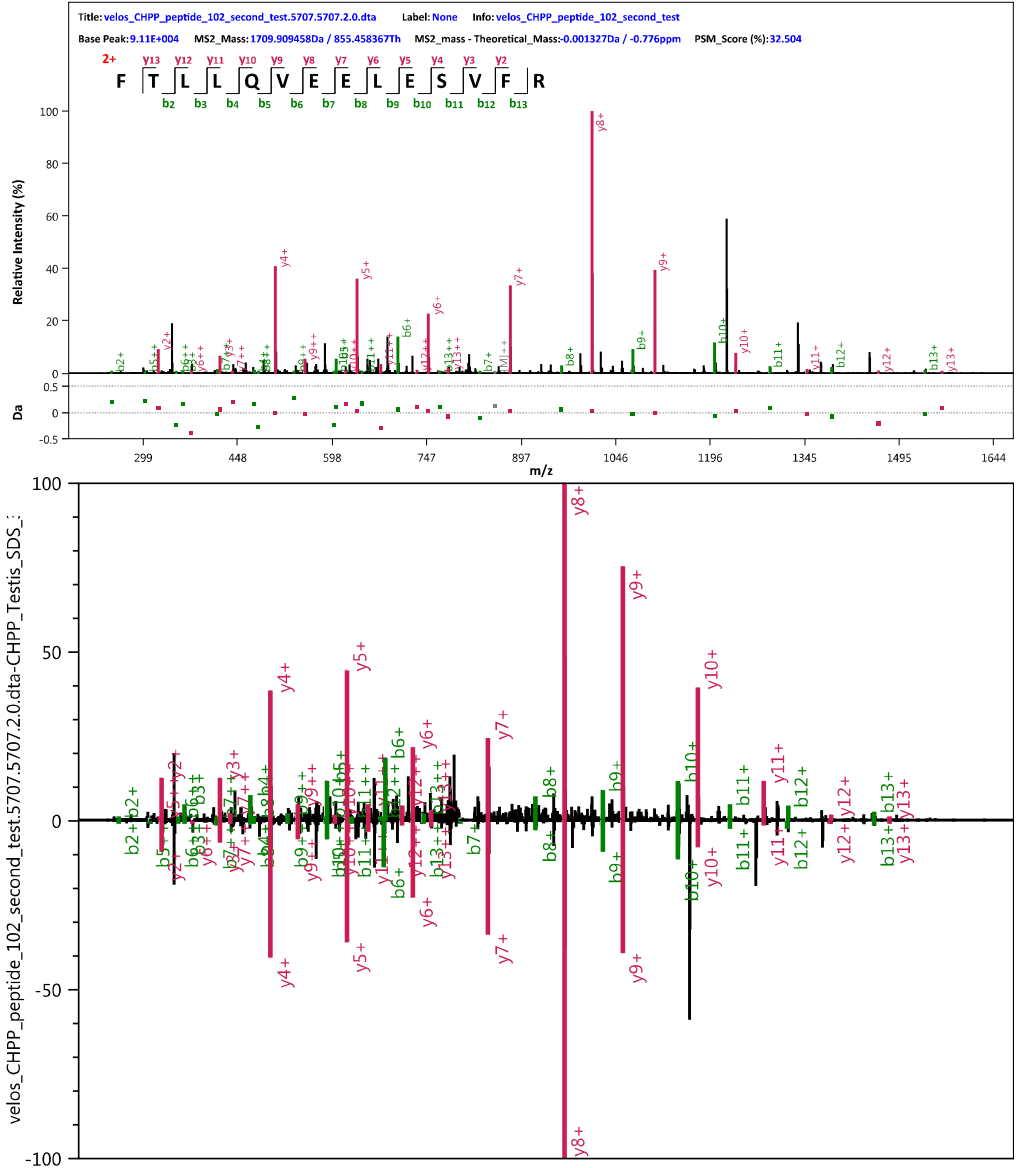


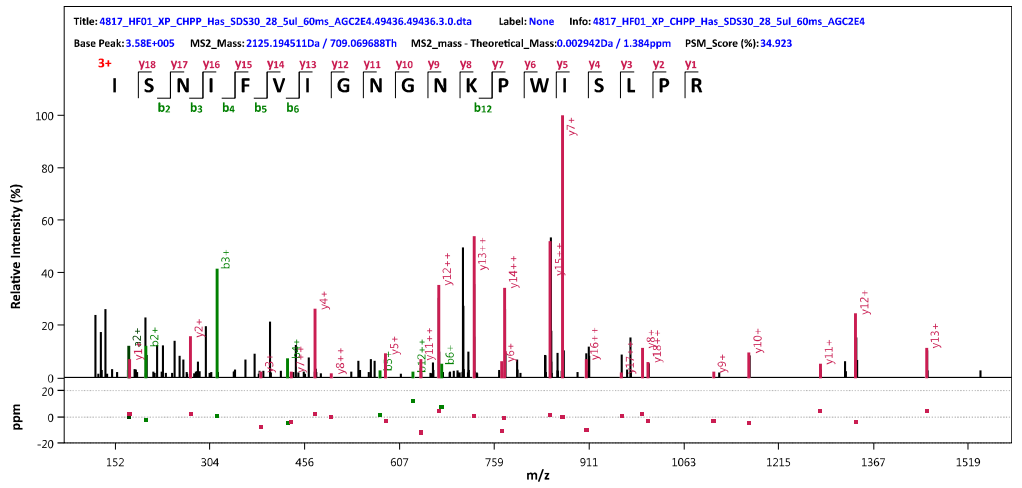

के
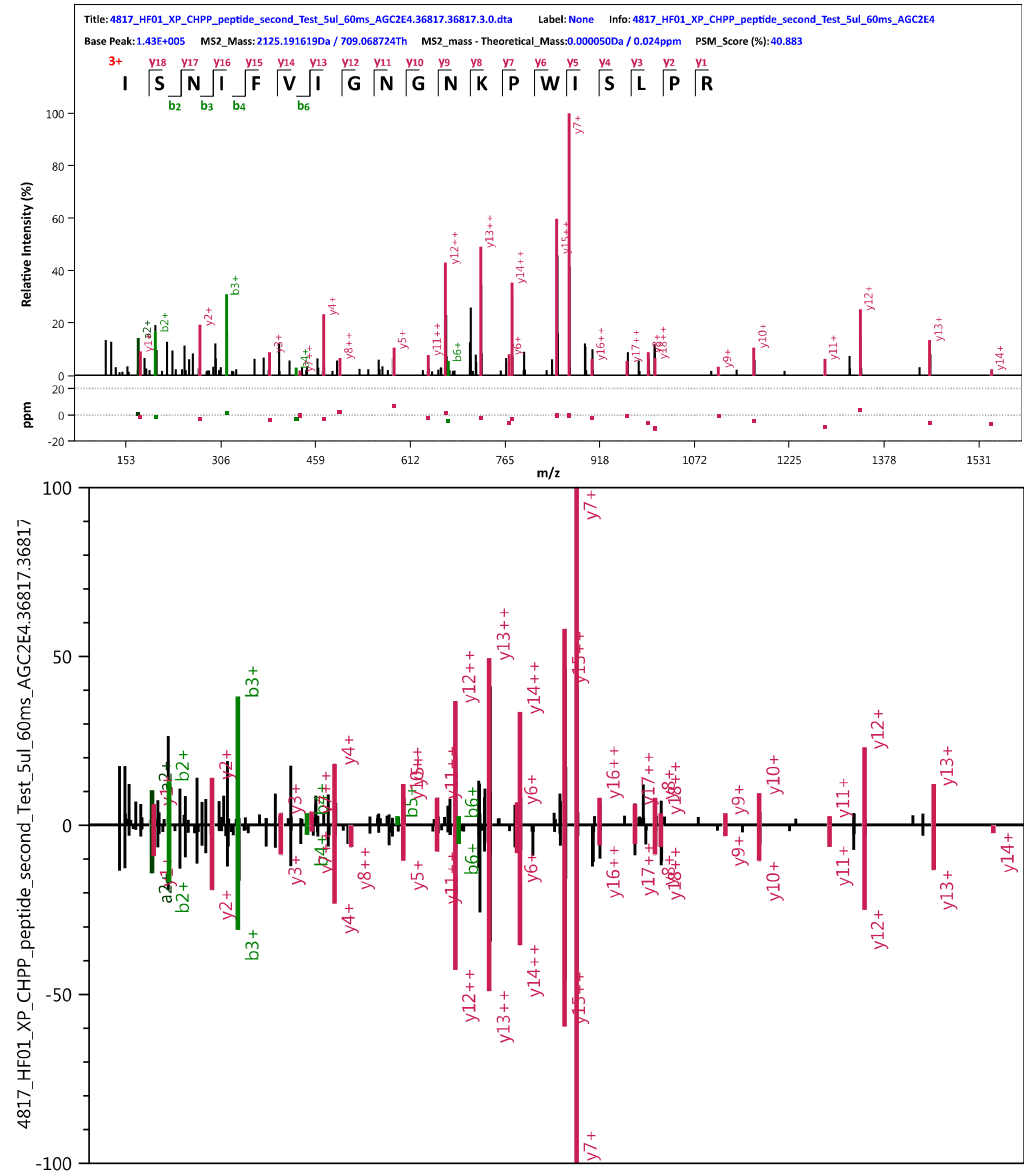
Title: 4817_HF01_XP_CHPP_Has_SDS10_25_5ul_60ms__AGC2E4.22029.22029.20.dta Label: None Info:4817_HF01_XP_CHPP_Has_SDS10_25_5ul_60ms_AGC2E4

Base Peak:2.30E+005 MS2_Mass: 1359.713066Da / 680.360171Th MS2_mass- Theoretical_Mass:0.001695Da / 1.247ppm PSM_Score (\%): 56.757

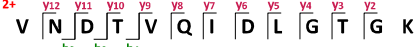

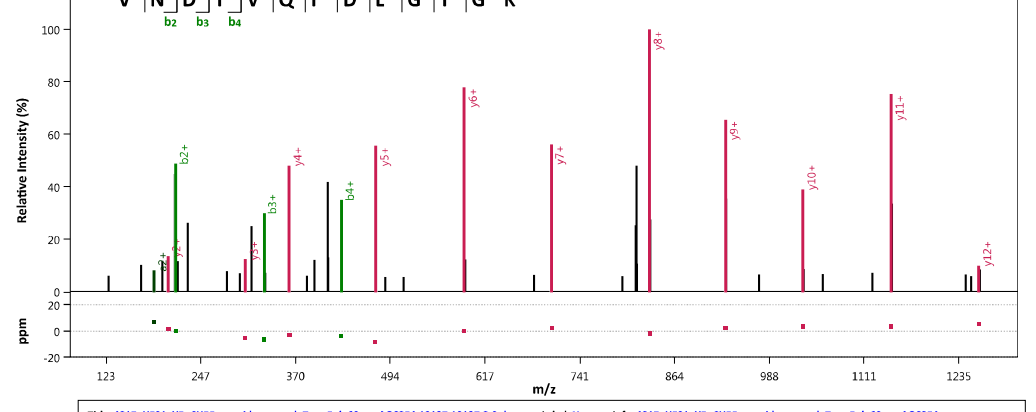

을

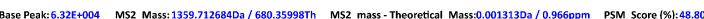

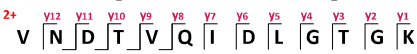
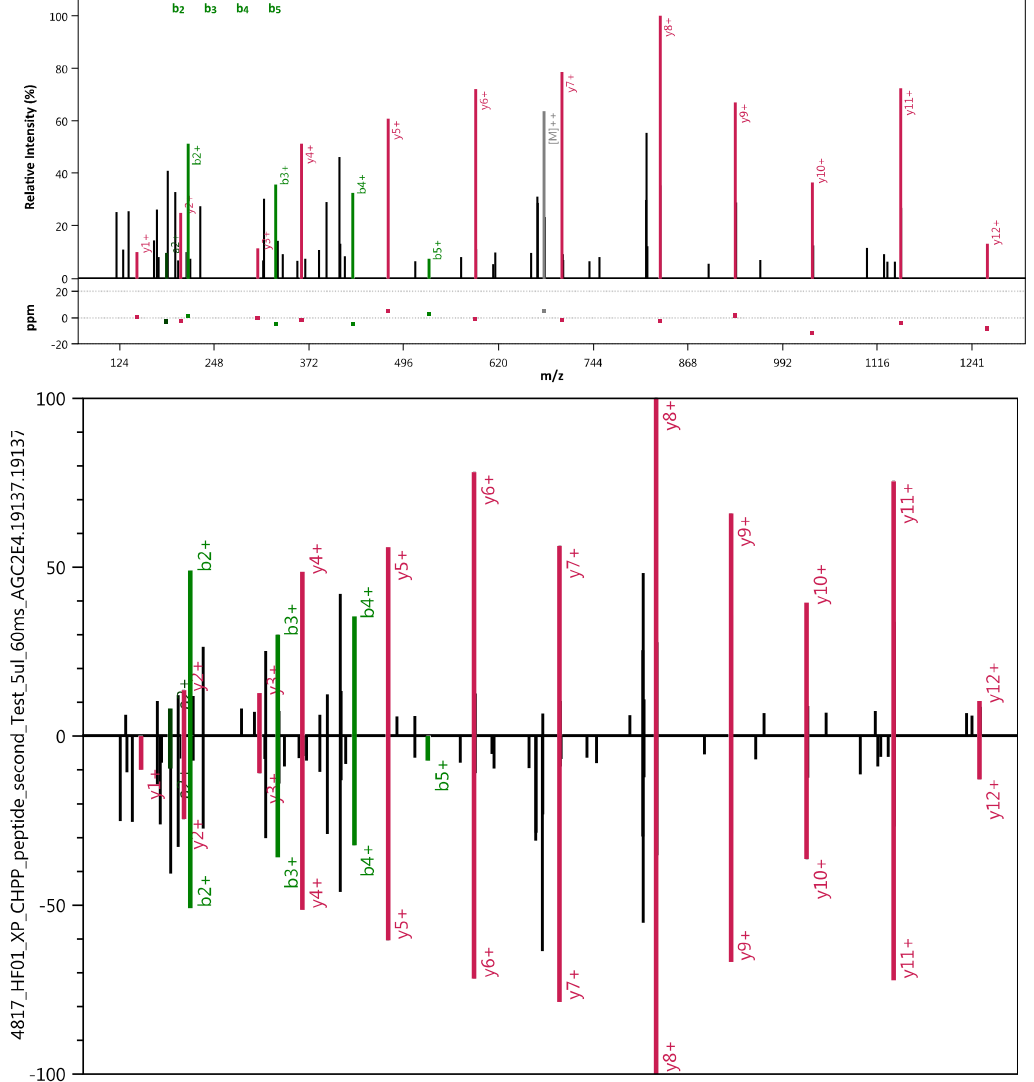
Title: 4817_HF01 XP CHPP Has SOS10 24 6ul 60ms AGC2E4.21098.21098.2.0.dta Label: None Info: 4817 HF01 XP CHPP Has SDS10 24 6ul 60 ms AGC2E4 Base Peak:1.27E+005 MS2_Mass:1359.725724Da / 680.3665Th MS2_mass - Theoretical_Mass:0.003121Da / 2.295ppm PSM_Score (\%):30.934

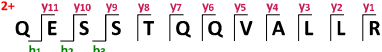

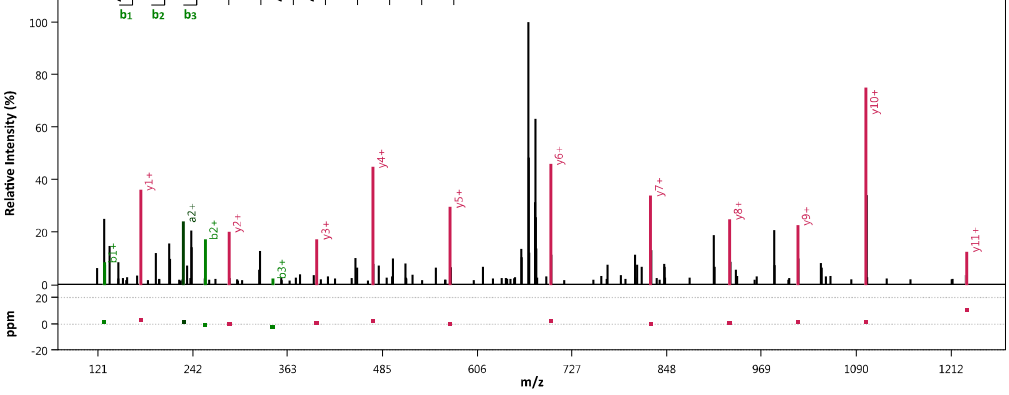

के
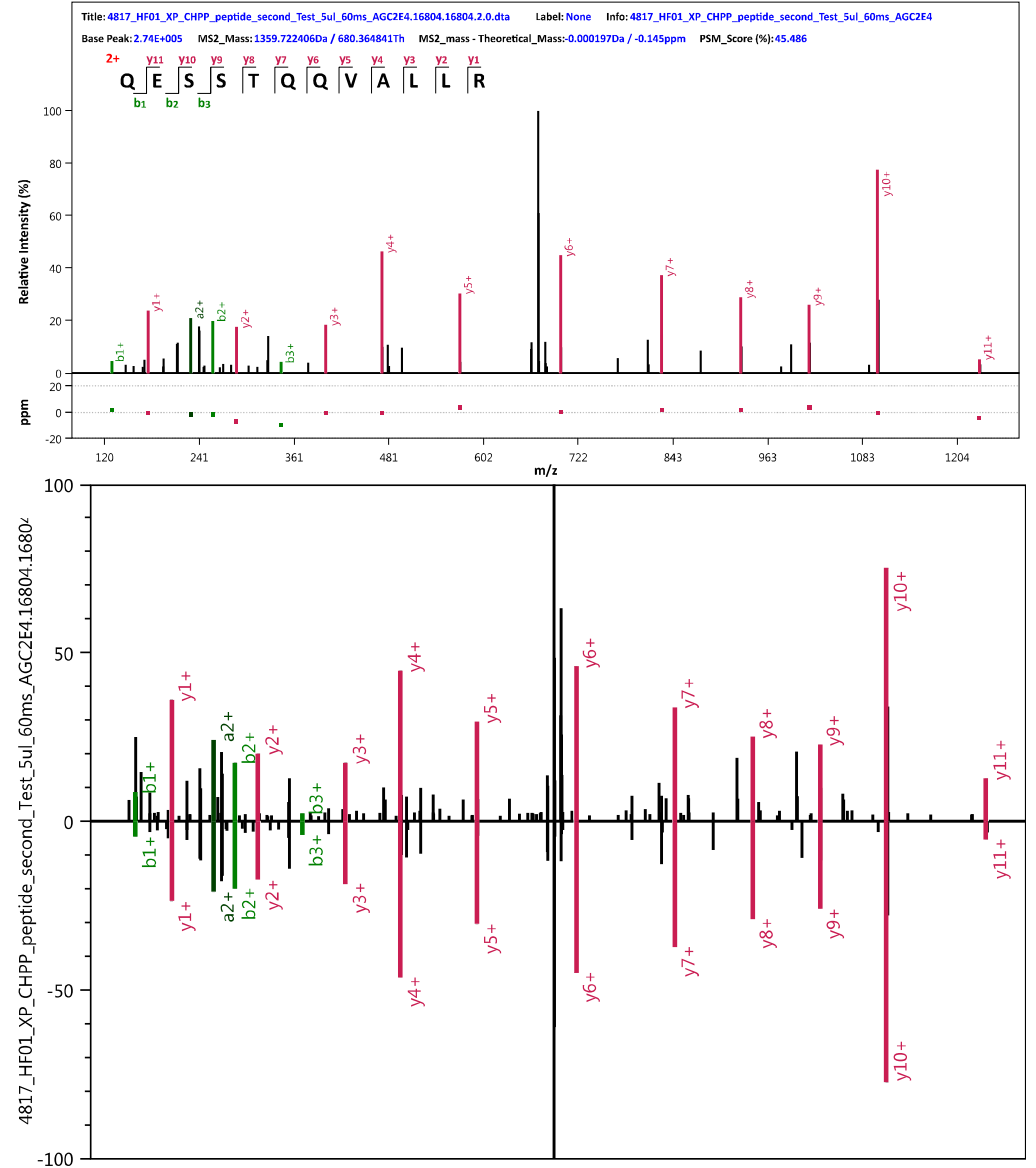
Titte: 4817_HF01_XP_CHPP_Has_SDS10_24_6ul_60ms__AGC2E4.19033.19033.3.1.dta Mods: 12,CCarboxymethyl[C](None); Label: None Info: 4817_HF01_XP_CHPP_Has_SDS10_24_6ul_60ms_AGC Base Peak:8.24E+004 MS2_Mass:2354.158824Da/ 785.391126Th MS2_mass - Theoretical_Mass:0.024304Da / 10.324ppm PSM_SCore (\%); :32.144

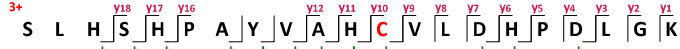
Base Peak: 1.71E+005 MS2_Mass: 2353.151764Da/ 785.055439Th MS2_mass - Theoretical_Mass:0.001259Da / 0.535ppm PSM_Score (\%): 32.912

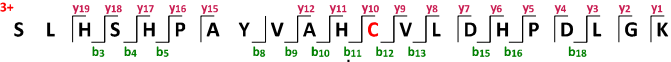

ס

言

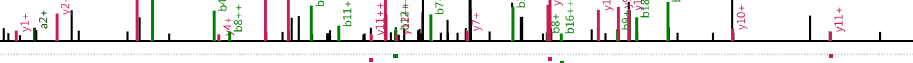
$0-$
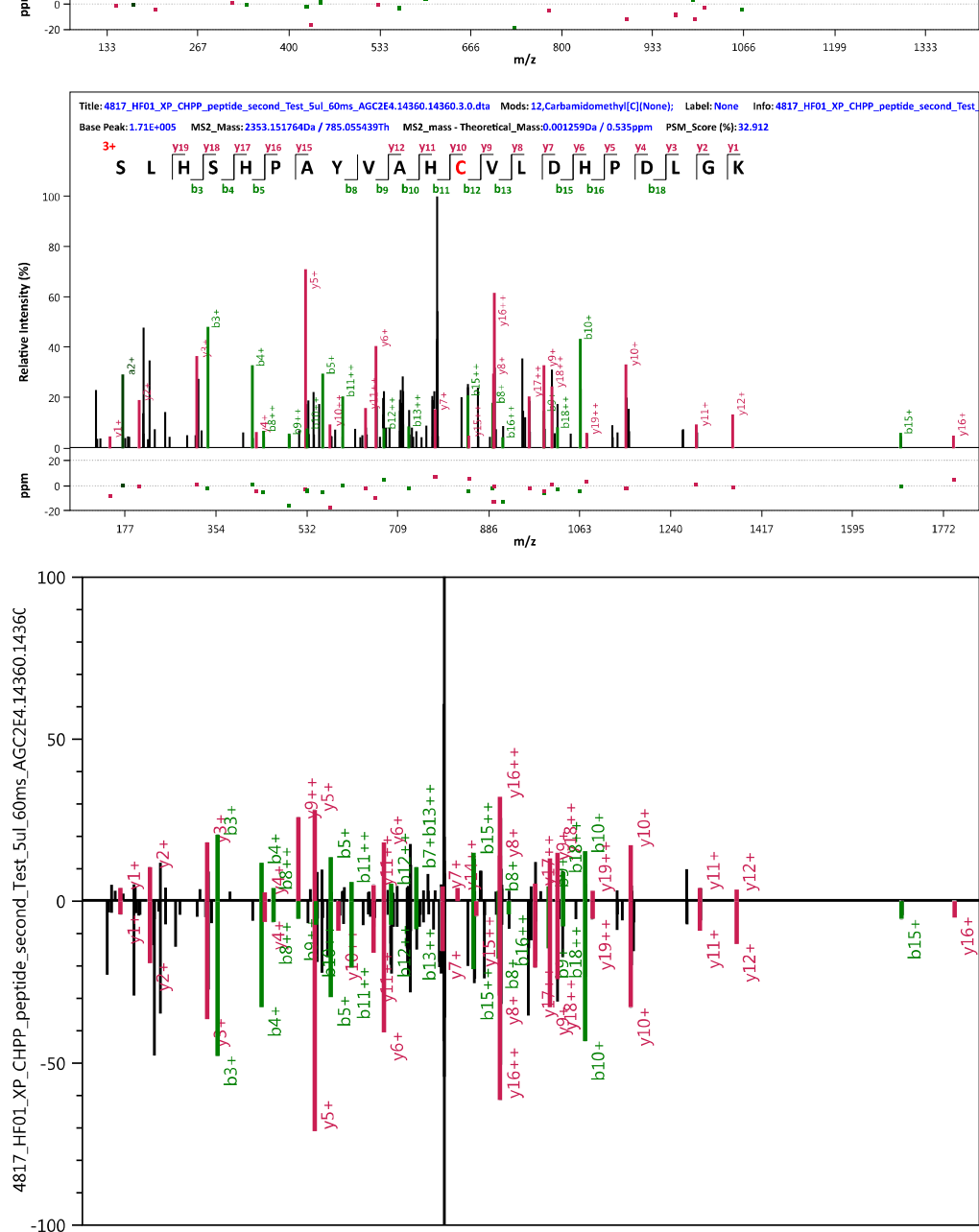

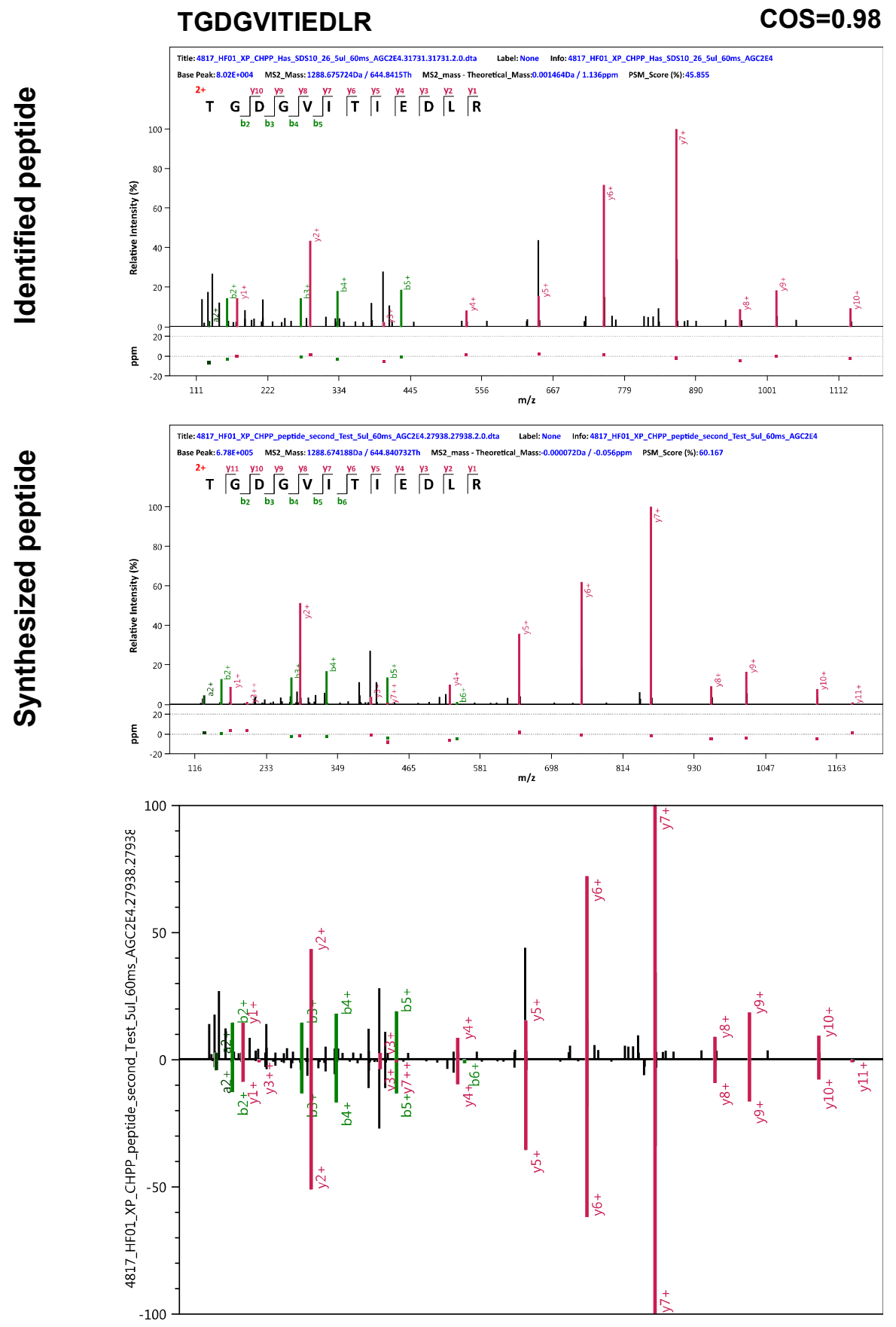

S-113 
Title: 4817_HF01_XP_CHPP_Has_SDS30_27_Sul_60ms_AGC2E4.27313.27313.2.0.dta Label: None Info: 4817_HF01_XP_CHPP_Has_SDS30_27_5U1_60ms_AGC2E4 Base Peak: 1.16E+005 MS2_Mass: 1671.741596Da / 836.374436Th MS2_mass - Theoretical_Mass:0.001892Da/ 1.132ppm PSM_Score (\%): 28.454

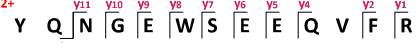

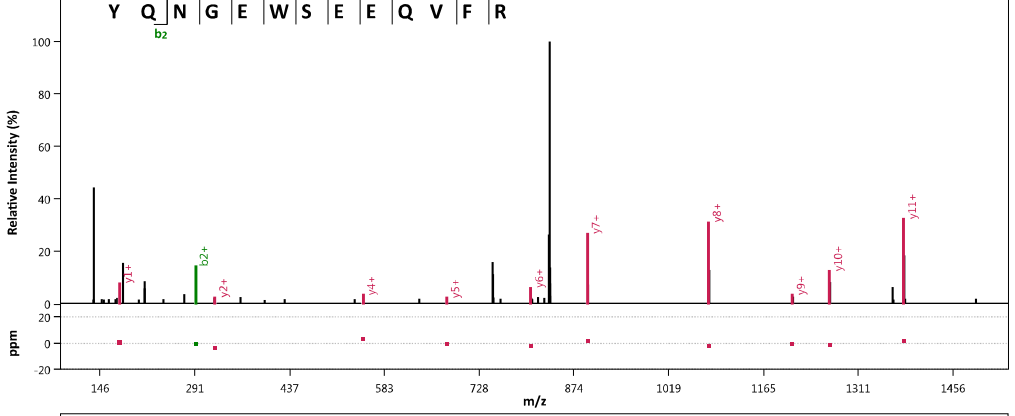

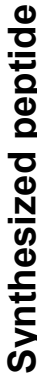

源 Base Peak:1.24E+008 MS2_Mass: 1671.741108Da / 836.374192Th MS2_mass - Theoretical_Mass:0.001404Da/0.840ppm PSM_score (\%): 56.867

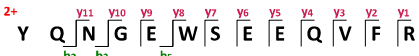
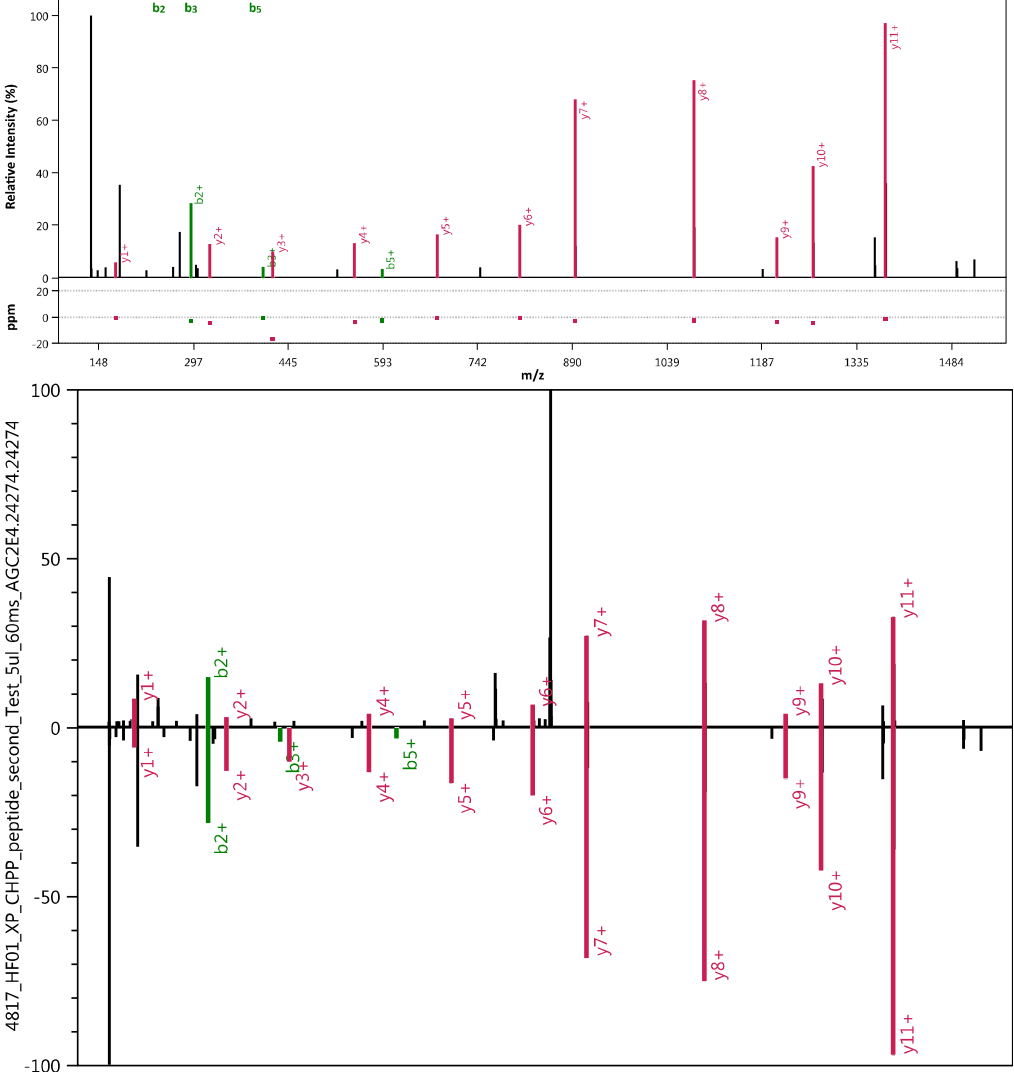
ESWEAAQETWEDK

COS $=0.99$
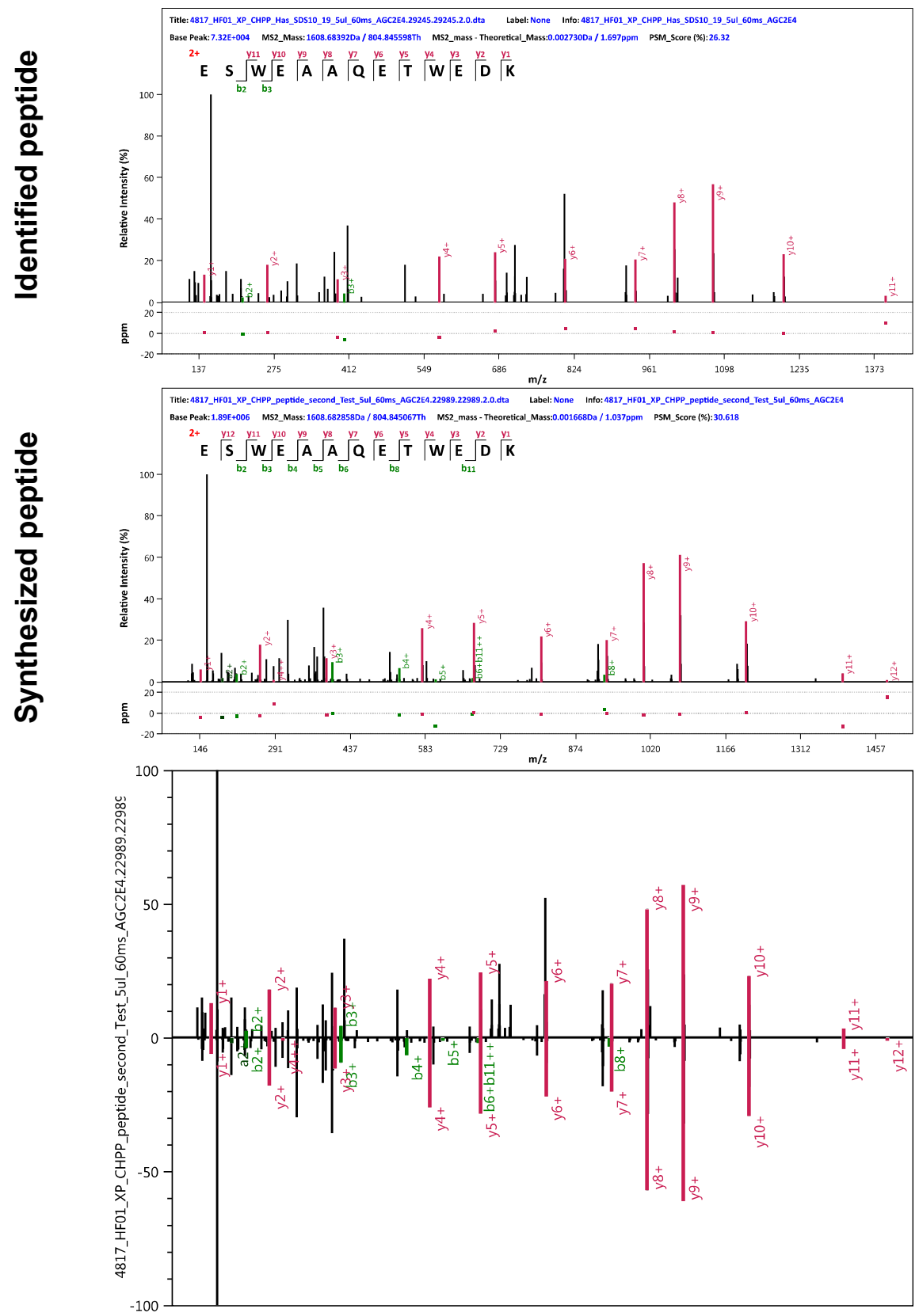

S-115 
Title: 4817_HF01_XP_CHPP_Has_SDS10_20_5ul_60ms_AGC2E4.18607.18607.2.0.dta Label:None Info: 4817_HF01_XP_CHPP_Has_SOS10_20_5ul_60ms_AGC2E4

Base Peak: 5.26E+004 MS2_Mass: 1152.592286Da / 576.799781Th MS2_mass - Theoretical_Mass:00.002810Da / 2.438ppm PSM_Score (\%):34.003

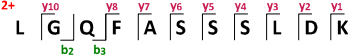

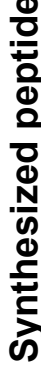
Base Peak: 2.23E+005 MS2_Mass: 1152.589328Da / 576.798302Th MS2_mass - Theoretical_Mass:-0.000148Da /-0.129ppm PSM_Score (\%): 43.535

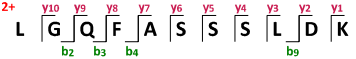
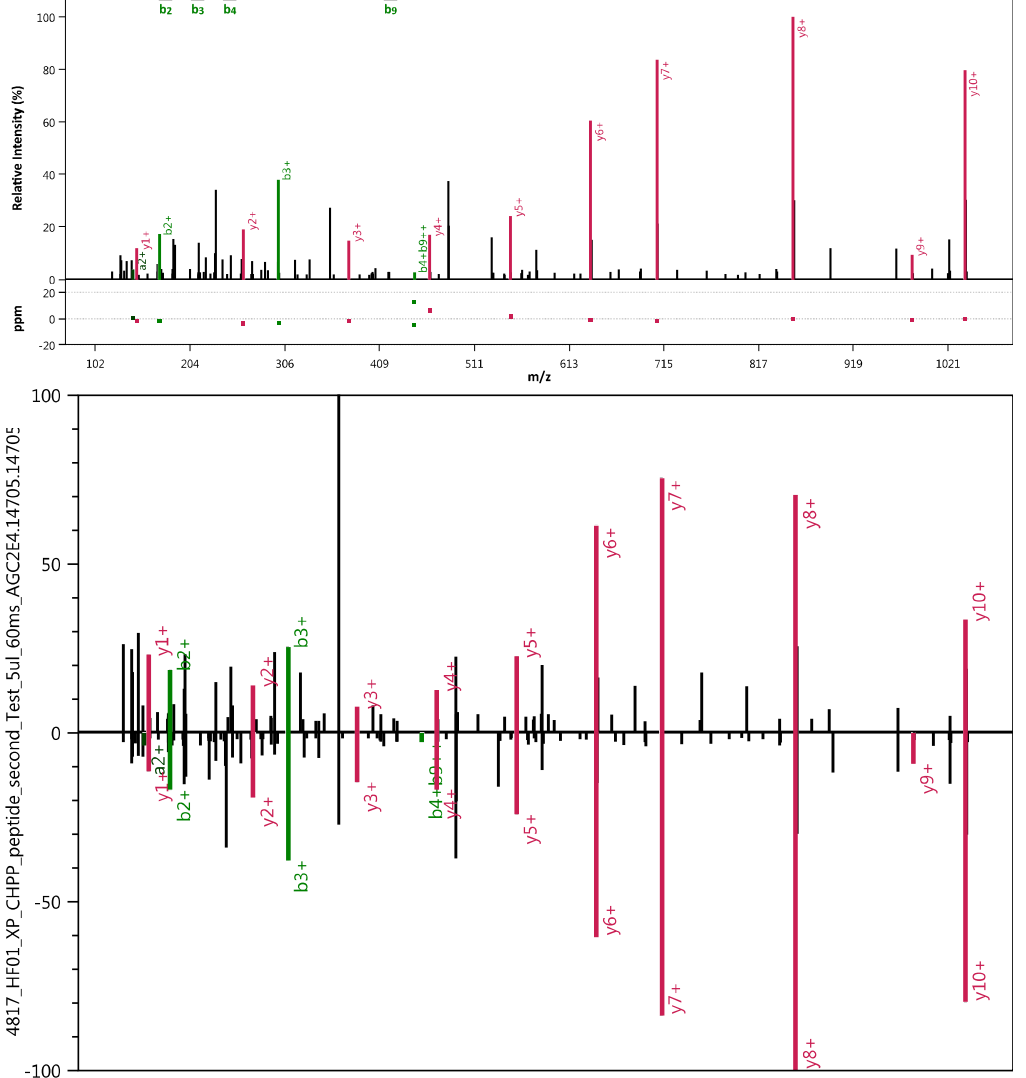


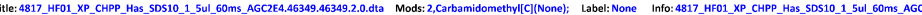
Tse Peak: 1.47E+005 MS2_Mass: 1712.822882Da / 856.915079Th MS2_mass - Theoretical_Mass:0.002956Da / 1.726ppm PSM_Score (\%):26.431

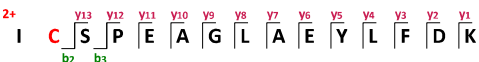

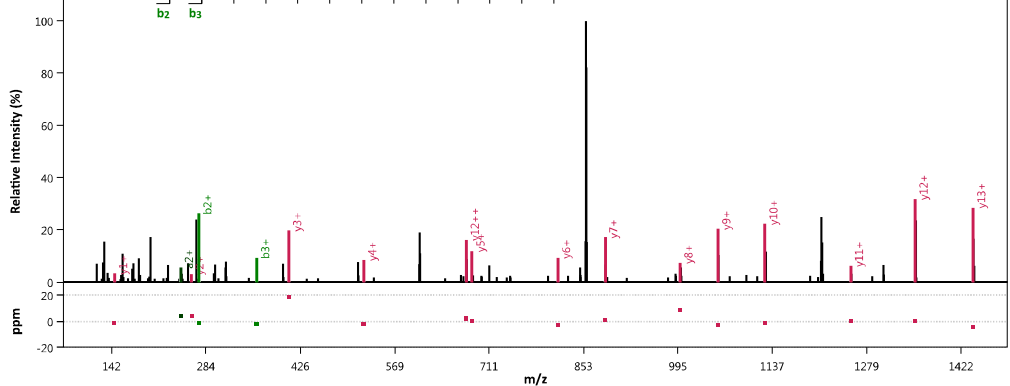

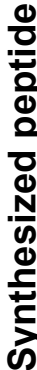

Title: 4817_HF01 XP_CHPP peptide_second Test_5ul_60ms_AGC2E4.36871.36871.2.0.dta Mods:2_Carbamidomethyl[CIINone); Label: None Info:4817_HF01_XP_CHPP_peptide_second_Test_s Base Peak: 5.38E+004 MS2_Mass: 1712.821228Da / 856.914252Th MS2_mass - Theoretical_Mas5:0.001302Da / 0.760ppm PSM_Score (\%):41.117

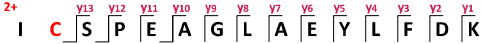
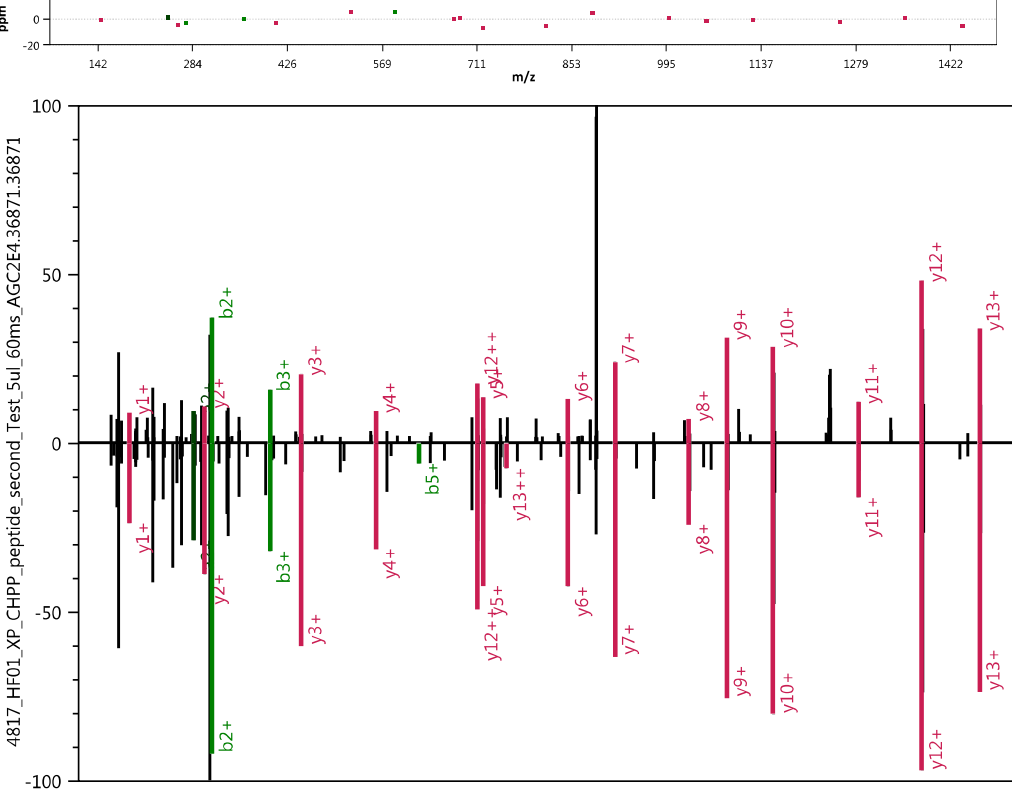


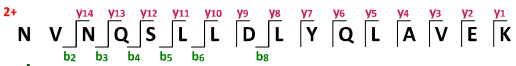

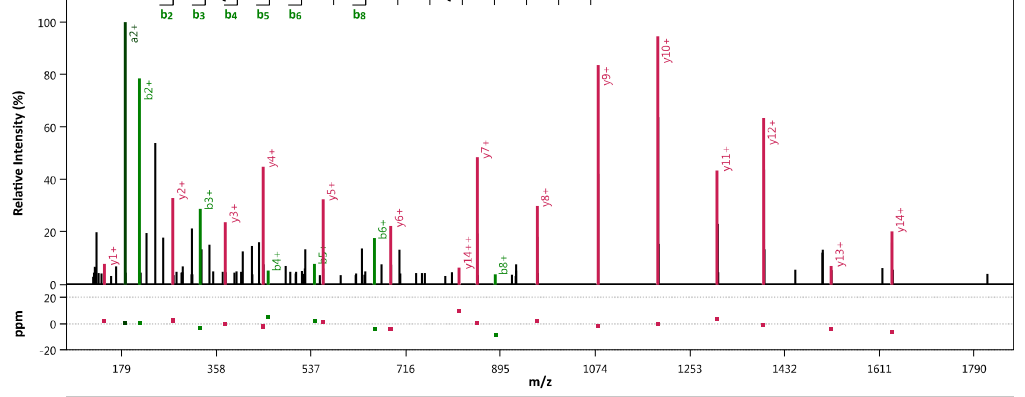

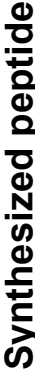

Titte: 4817_HF01_XP_CHPP_Peptide_second_Test_5ul_60ms_AGC2E4440008.40008.2.0.dta Label: None Info: 4817_HF01_XP_CHPP_peptide_second_Test_5ul_60ms_AGC2E4

k:2.15E+006 MS2_Mass: 1846.990084Da / 923.99868Th MS2 mass - The

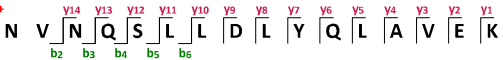
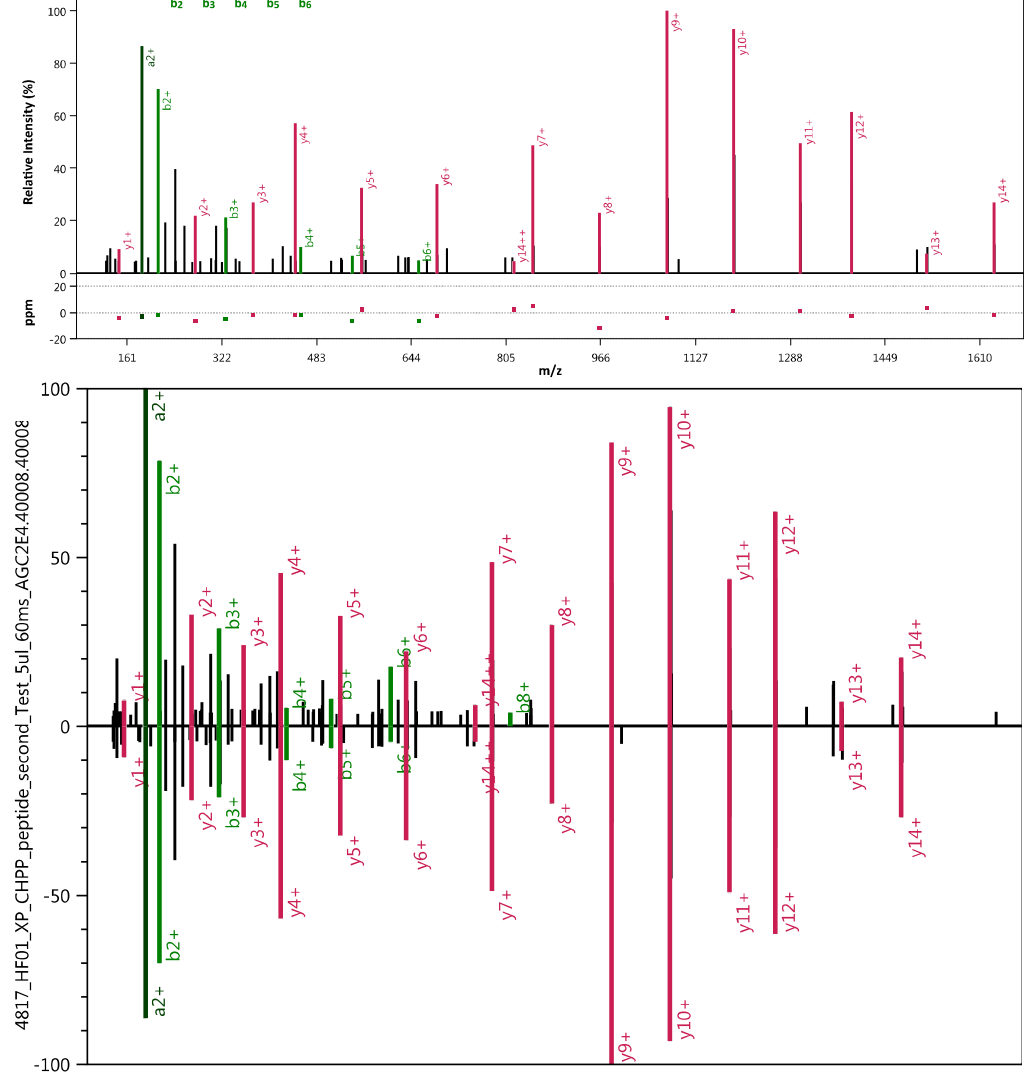


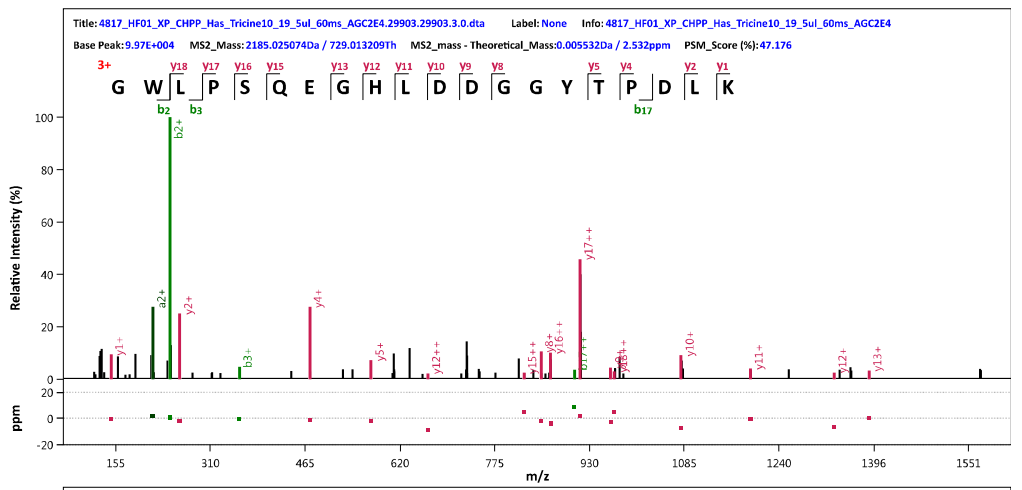

के
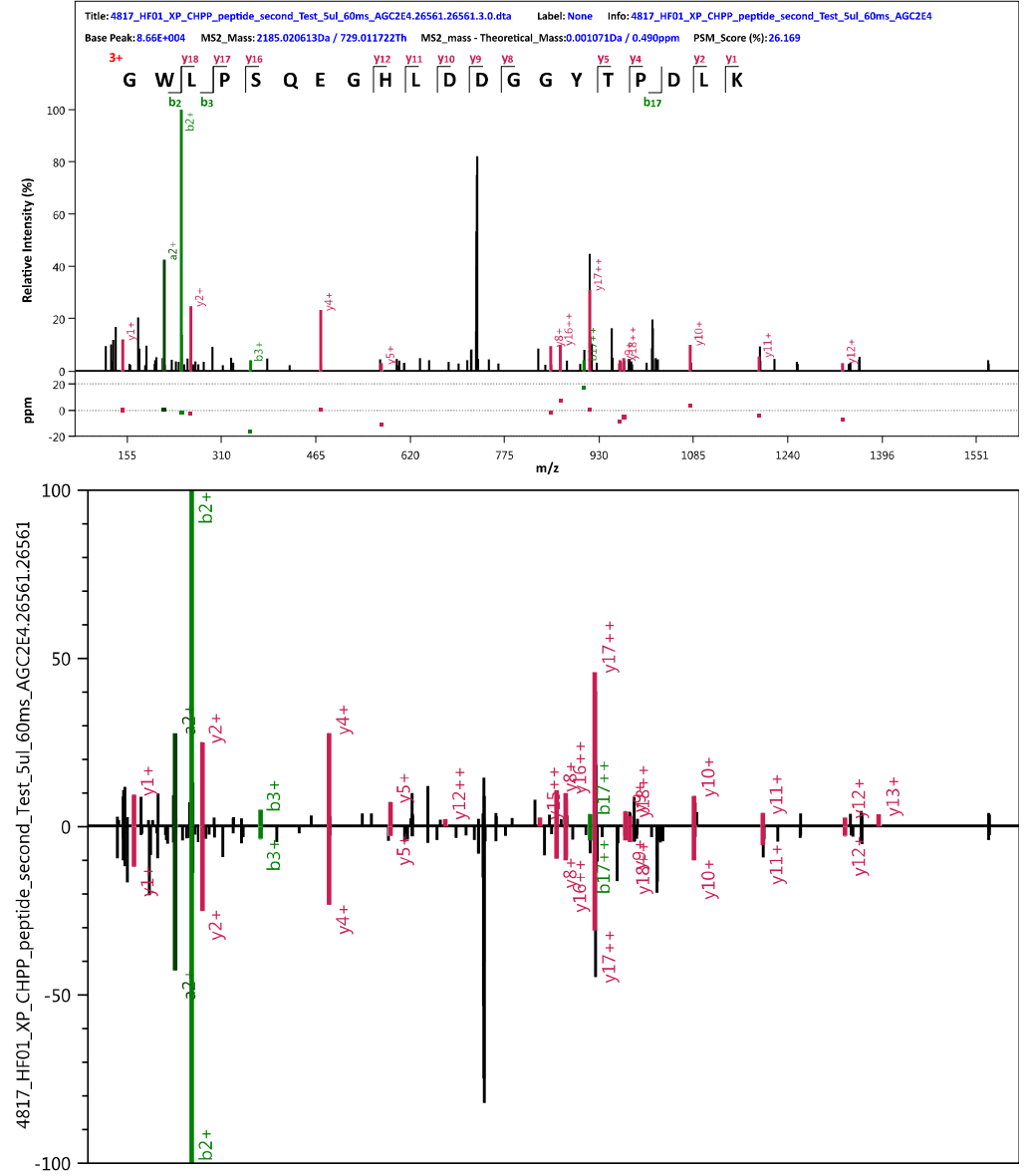
Thle:CHPP_TESTIS_Tricine_3012.1140.1140.2.0.dta Label: None Info: CHPP_TESTIS_Tricine_3012

ase Peak:2.40E+005 MS2_Mass: 1058.512388Da / 529.759832Th MS2_mass - Theoretical_Mass:0.001158Da / 1.094ppm PSM Score (\%): 45.356

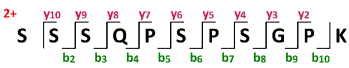

19

임

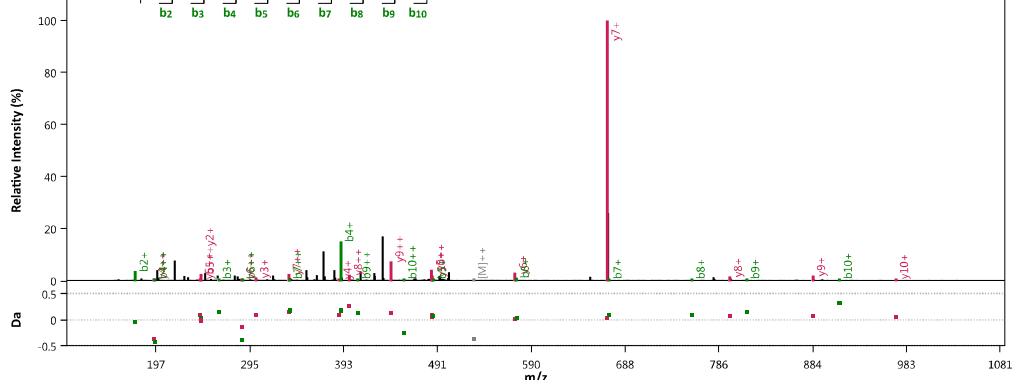

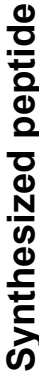
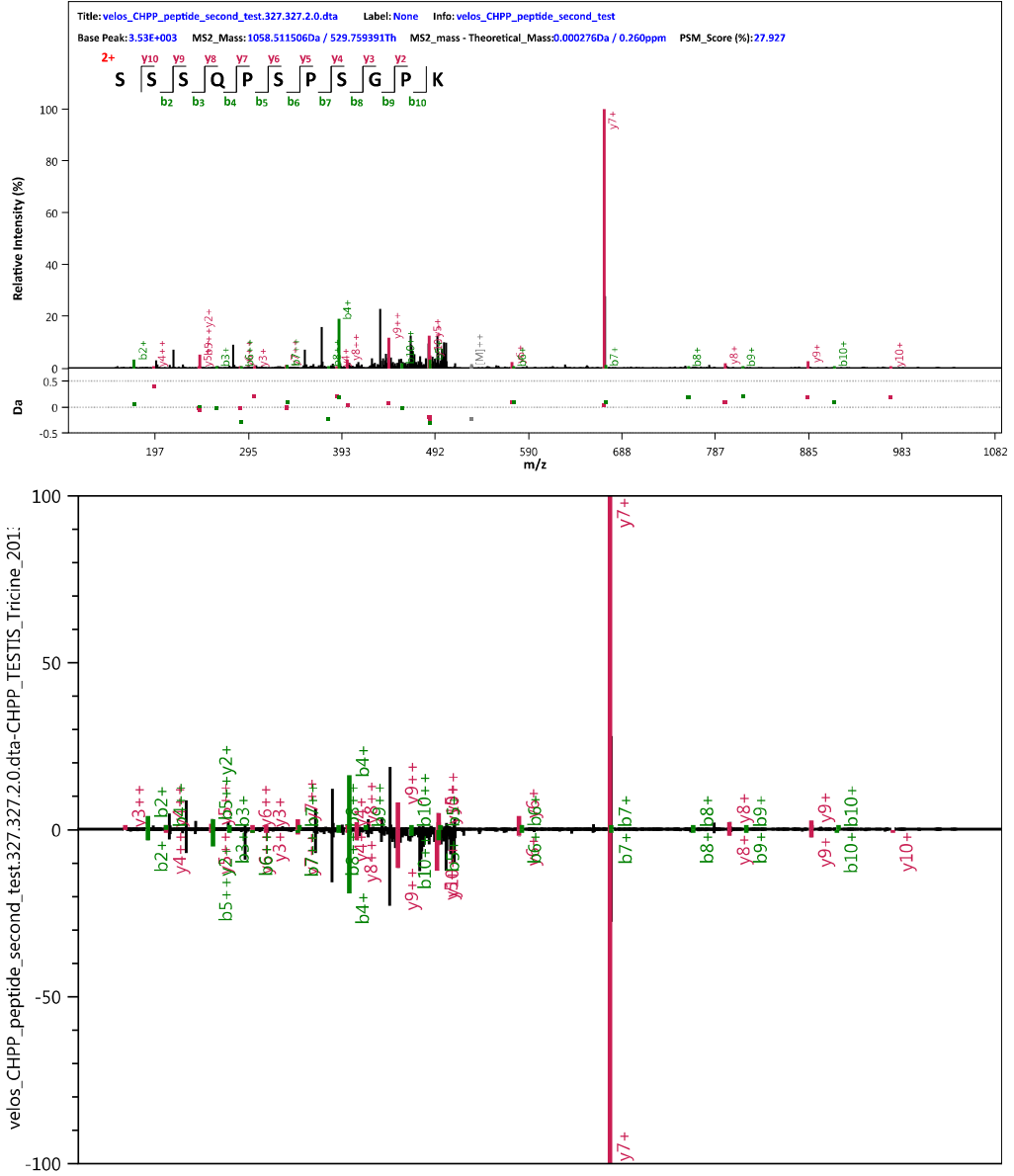


\section{GLNSSYDQQEYIGR}

$\operatorname{COS}=0.98$

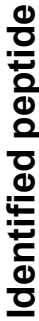

Title: CHPP Testis_SDS 1002.13926.13926.2.0.dta Label: None Info: CHPP Testis SDS 1002

Base Peak: 5.13E+003 MS2_Mass: 1629.751711Da/815.379494Th MS2_mass-Theoretical_Mass:0.001444Da / 0.886ppm PSM_Score (\%): 18.044

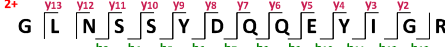

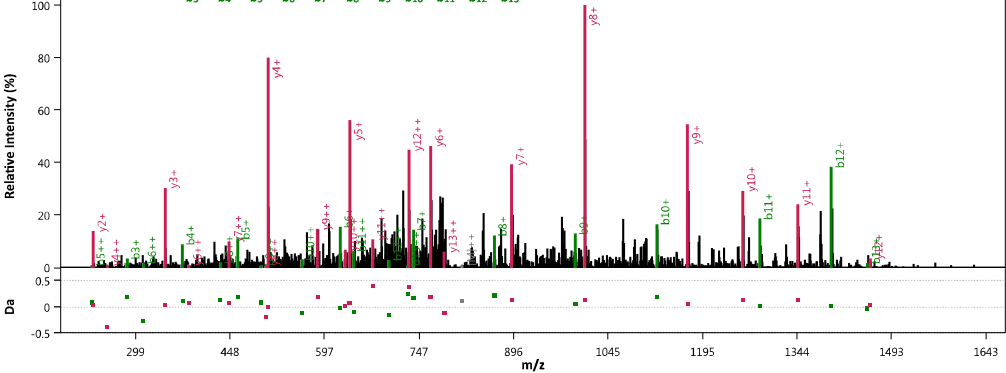

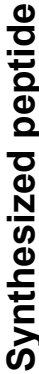
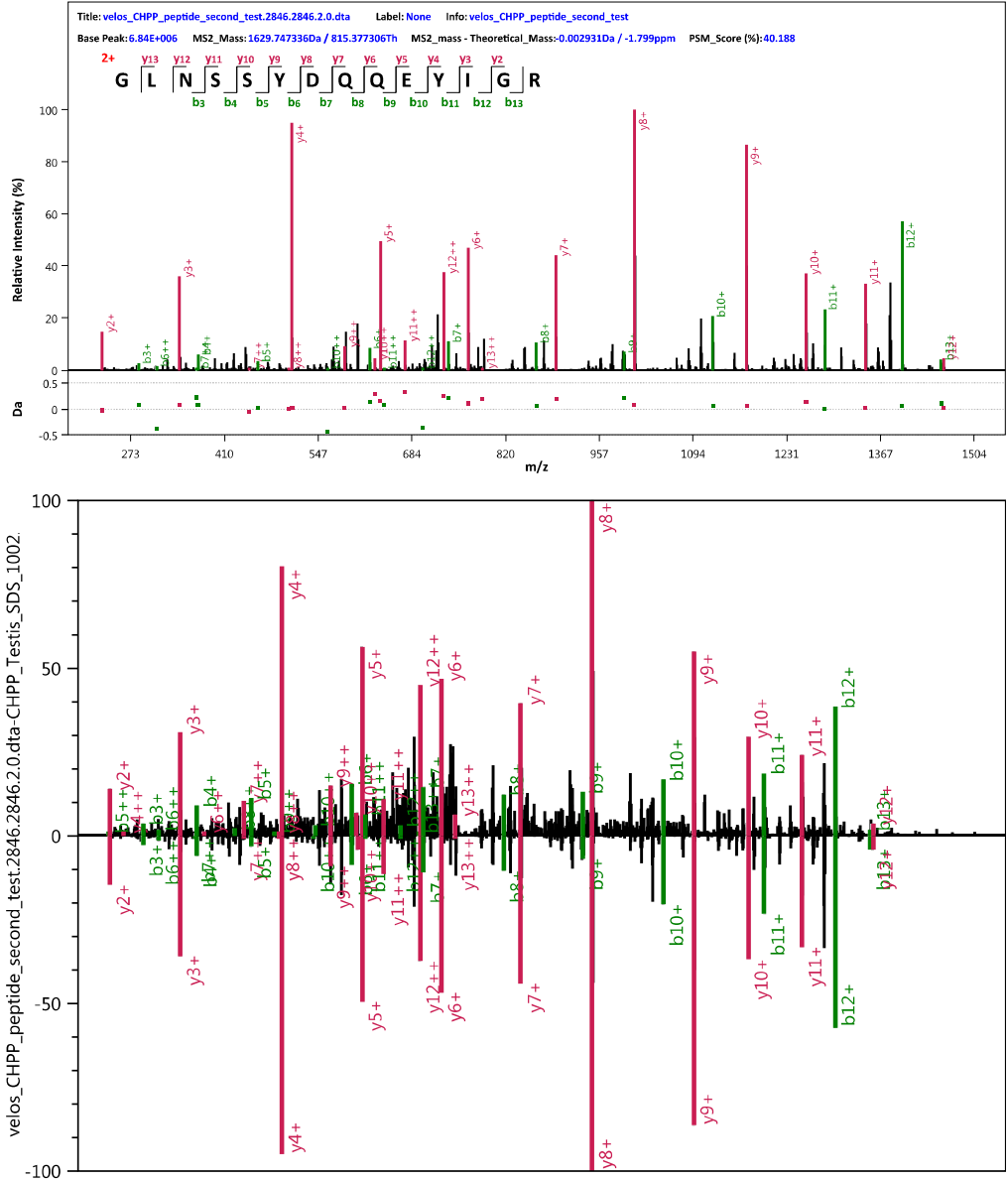
Title: CHPP_Testis__SD__1002.1785.1785.2.0.dta Label: None Info:CHPP_Testis__SDS_1002

Base Peak:2.89E+003 MS2_Mass: 1699.72937Da /850.368323Th MS2_mass - Theoretical_Mass:0.004141Da/ 2.436ppm PSM_Score (\%):26.257

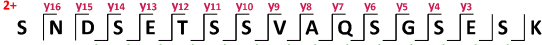

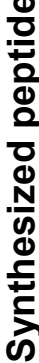

:

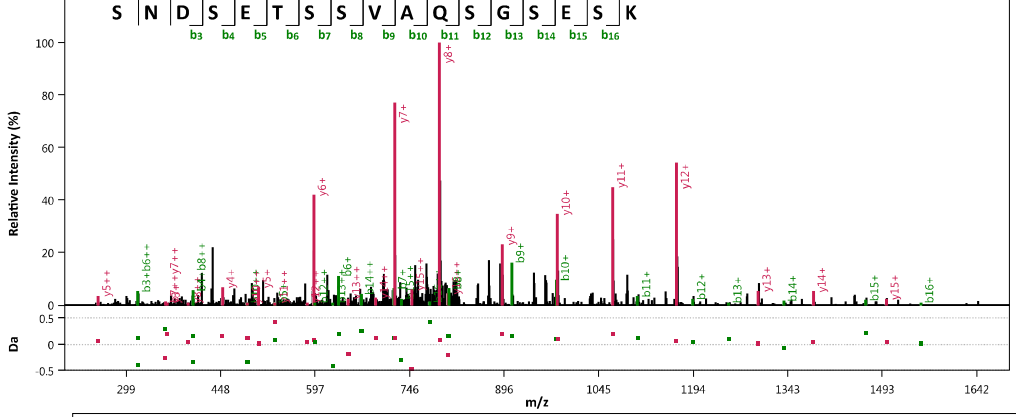

Base Peak:7.44E+003 MS2 Mass: 1699.726246Da / 850.366761Th MS2 mass - Theoretical_Mass:0.001017Da / $0.598 \mathrm{ppm}$ PSM Score (\%): 25.767

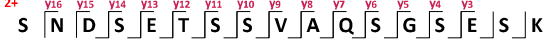
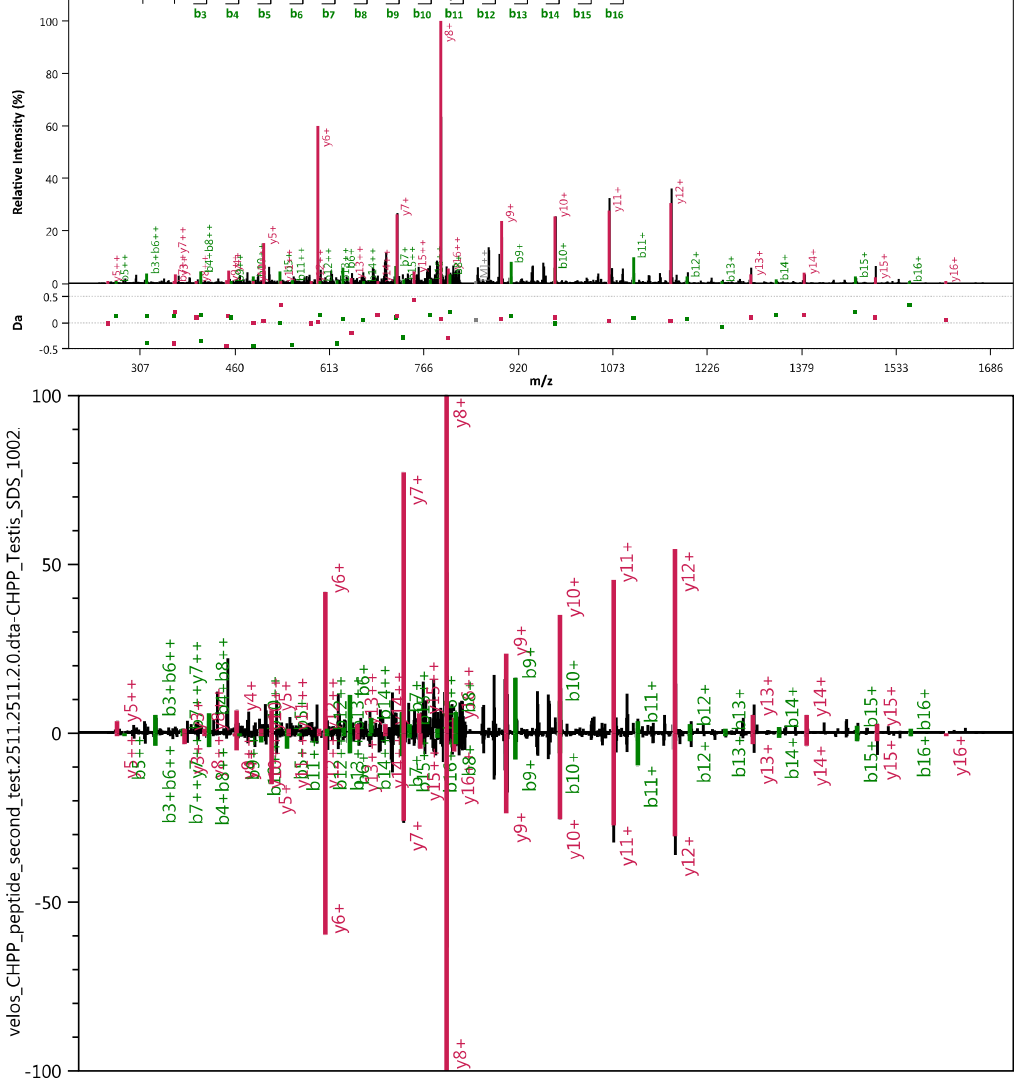

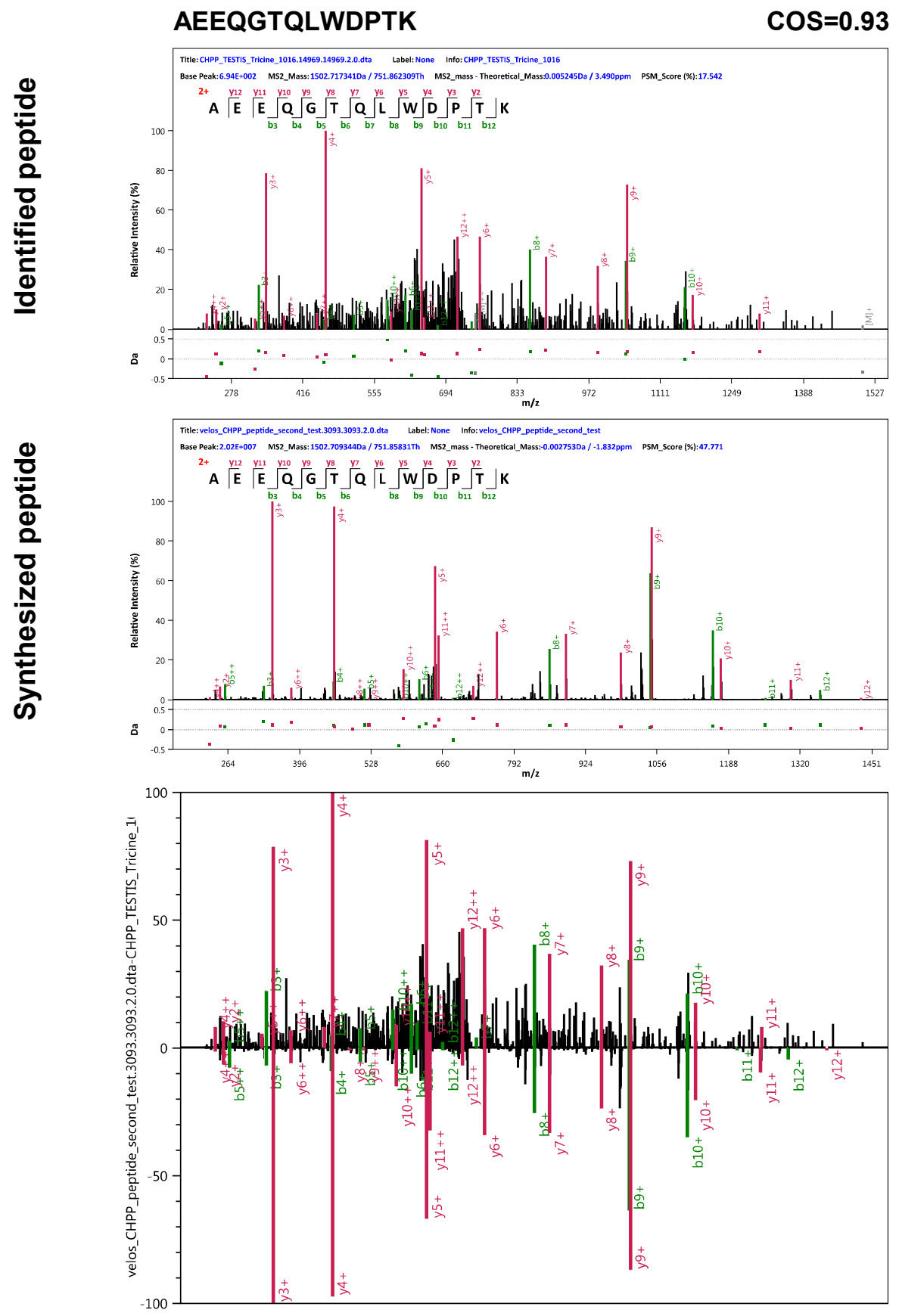

S-123 


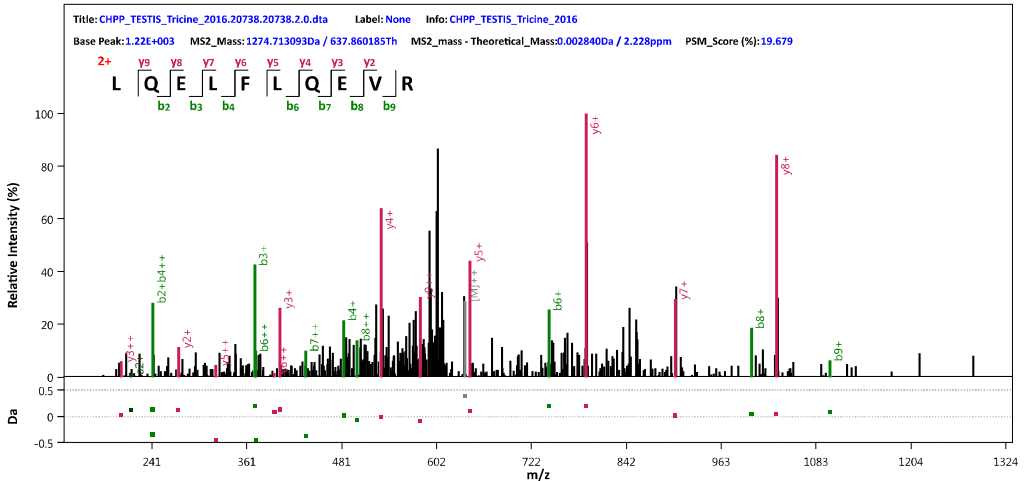

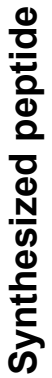
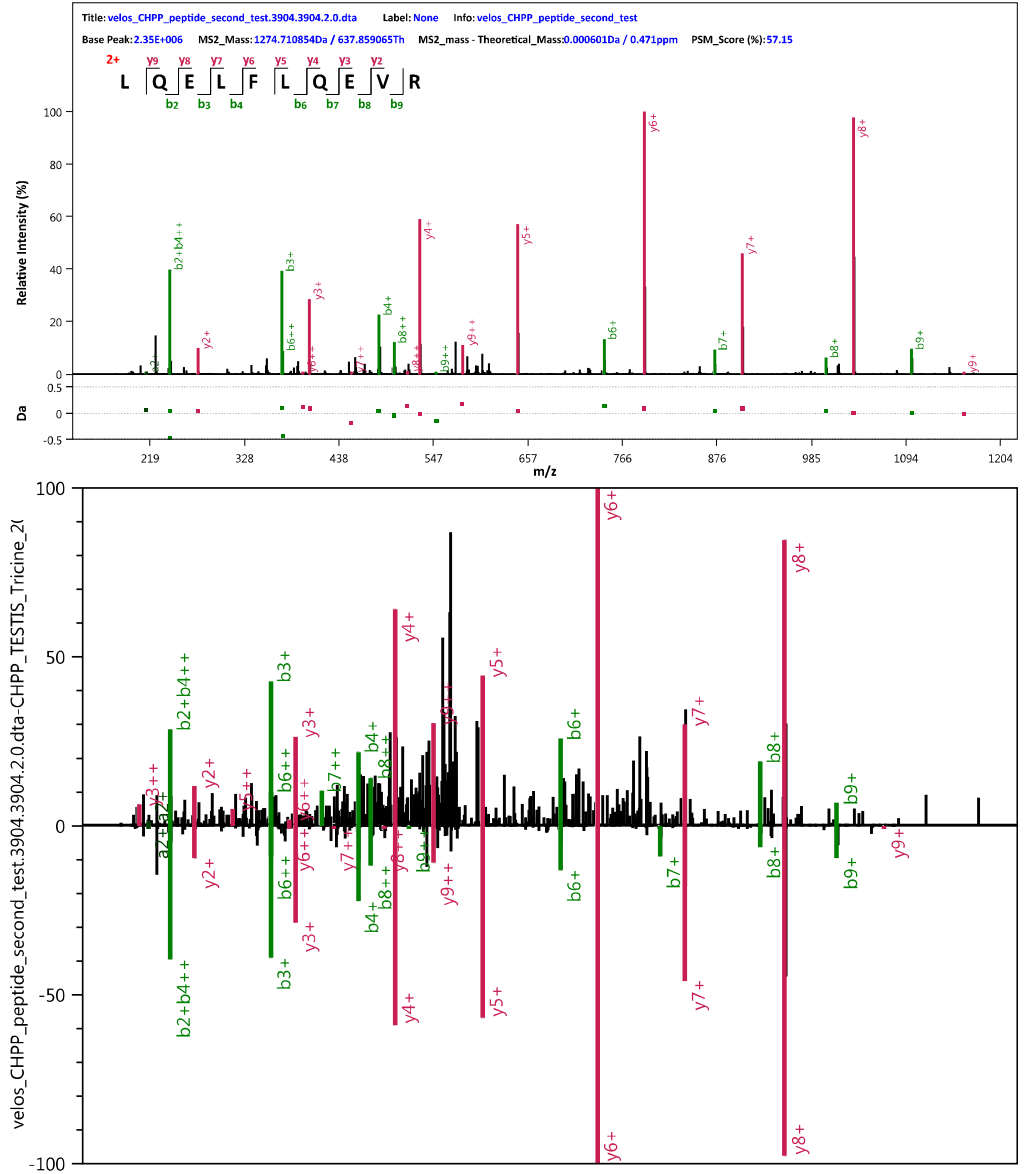

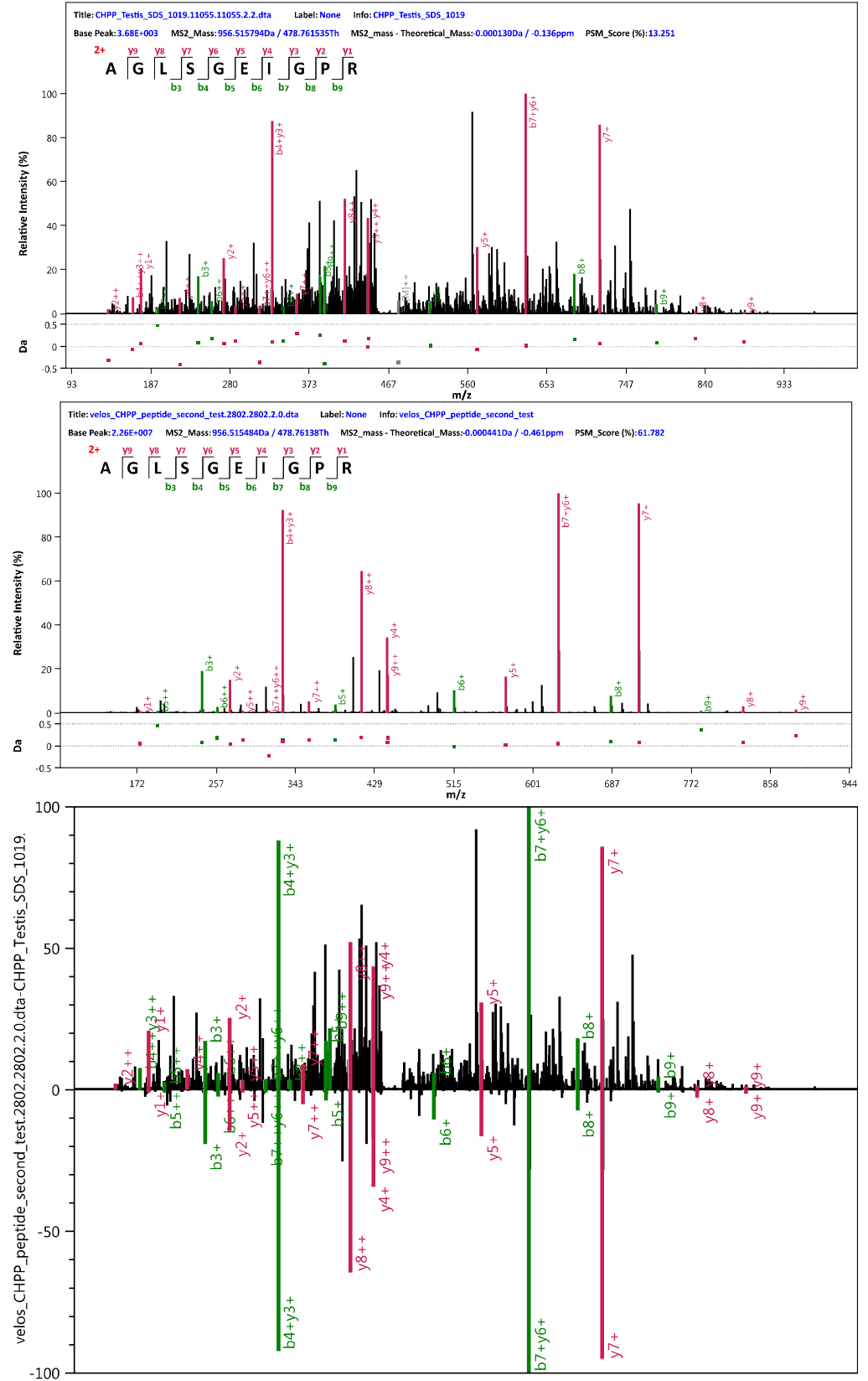
Title:CHPP_Testis_SDS_1019.30108.30108.2.2.dta Mods: 11,Carboxymethyl(C)(None); Label: None Info: CHPP_Testis_SDS_1019

gase Peak: 1.51E+003 MS2_Mass: 1741.919683Da / 871.46348Th MS2_mass - Theoretical_Mass:0021569Da / 12.383ppm PSM_Score (\%): 12.552

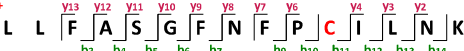

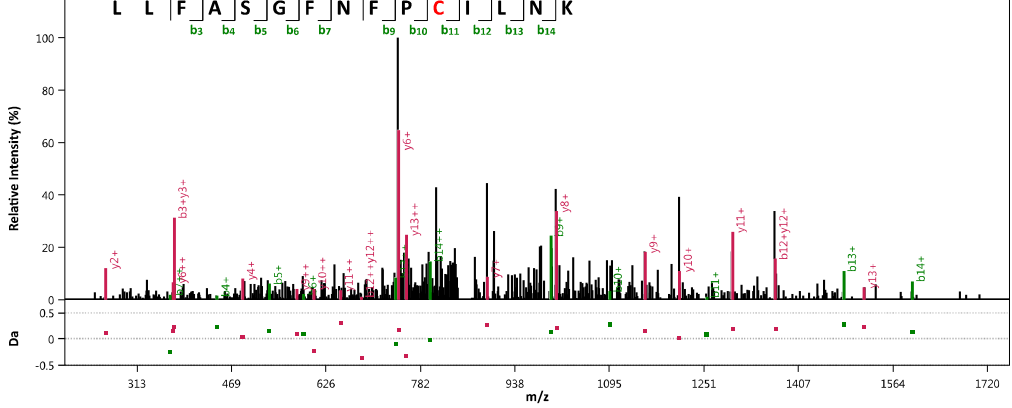

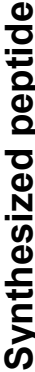
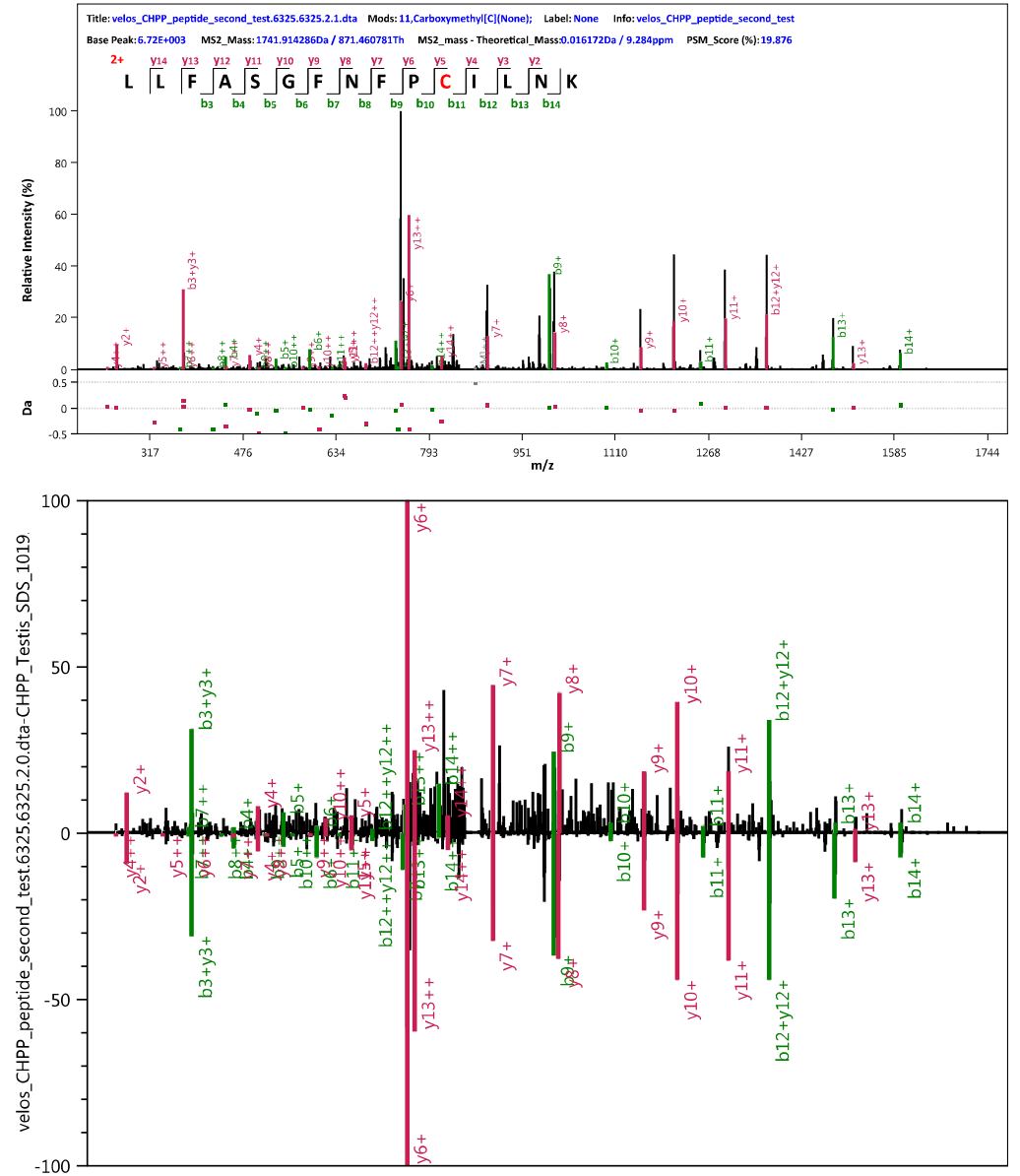